\title{
Identification of potential key genes and pathway linked with sporadic Creutzfeldt-Jakob disease based on integrated bioinformatics analyses
}

Basavaraj Vastrad $^{1}$, Chanabasayya Vastrad ${ }^{* 2}$, Iranna Kotturshetti

1. Department of Biochemistry, Basaveshwar College of Pharmacy, Gadag, Karnataka 582103, India.

2. Biostatistics and Bioinformatics, Chanabasava Nilaya, Bharthinagar, Dharwad 580001, Karanataka, India.

3. Department of Ayurveda, Rajiv Gandhi Education Society`s Ayurvedic Medical College, Ron, Karnataka 562209, India.

* Chanabasayya Vastrad

channu.vastrad@gmail.com

Ph: +919480073398

Chanabasava Nilaya, Bharthinagar,

Dharwad 580001, Karanataka, India 


\begin{abstract}
Sporadic Creutzfeldt-Jakob disease (sCJD) is neurodegenerative disease also called prion disease linked with poor prognosis. The aim of the current study was to illuminate the underlying molecular mechanisms of sCJD. The mRNA microarray dataset GSE124571 was downloaded from the Gene Expression Omnibus database. Differentially expressed genes (DEGs) were screened. Pathway and GO enrichment analyses of DEGs were performed. Furthermore, the protein -protein interaction (PPI) network was predicted using the IntAct Molecular Interaction Database and visualized with Cytoscape software. In addition, hub genes and important modules were selected based on the network. Finally, we constructed target genes - miRNA regulatory network and target genes - TF regulatory network. Hub genes were validated. A total of 891 DEGs 448 of these DEGs presented significant up regulated, and the remaining 443 down regulated were obtained. Pathway enrichment analysis indicated that up regulated genes were mainly linked with glutamine degradation/glutamate biosynthesis, while the down regulated genes were involved in melatonin degradation. GO enrichment analyses indicated that up regulated genes were mainly linked with chemical synaptic transmission, while the down regulated genes were involved in regulation of immune system process. hub and target genes were selected from the PPI network, modules, and target genes - miRNA regulatory network and target genes - TF regulatory network namely YWHAZ, GABARAPL1, EZR, CEBPA, HSPB8, TUBB2A and CDK14. The current study sheds light on the molecular mechanisms of sCJD and may provide molecular targets and diagnostic biomarkers for sCJD.

Key words: sporadic Creutzfeldt-Jakob disease; ToppGene database; proteinprotein interaction (PPI) network; bioinformatics tools; gene ontology enrichment analysis
\end{abstract}

\title{
Introduction
}


medRxiv preprint doi: https://doi.org/10.1101/2020.12.21.20248688; this version posted December 24, 2020. The copyright holder for this

Prion diseases of human beings are linked with the aggregation in the brain of an abnormal, slightly protease-resistant isoform, of cellular prion protein (PrPC) [1]. Prion diseases are neurodegenerative diseases that affect human beings [2]. However, dissimilar with the most common diseases of this group, such as Alzheimer's disease, Lewy body disorders and frontotemporal dementias [3]. Sporadic Creutzfeldt-Jakob disease (sCJD) is the most prevalent human prion disease occurs through unknown origin [4]. sCJD is considered as a gene-related disease [5]. Expanding confirmation also has demonstrated that multiple genes and cellular pathways participate in the occurrence and advancement of sCJD [6-7]. Alteration and polymorhisum in prion protein was associated with progression of sCJD [8-9]. To date, a deficiency of understanding the accurate molecular mechanisms underlying SCJD development limits the ability to treat advanced disease. In recent years, microarray technology is now undergoing a revolution, which could be used on tissue or blood samples to diagnose key biomarkers and novel pathways in individual person [10-11]. Therefore, further knowledge of molecular mechanism associated in genetic expression disorder of sCJD, which is extremely key for the future advancement of diagnosis and treatment could be learned through this latest technology. Gene expression analysis [12] now is a popular approach to analyze the expression changes of gene in the advancement and progression of sCJD, comprehensively. In this current study, we downloaded the original gene expression dataset (GSE124571) from the Gene Expression Omnibus (GEO), which is repository leads to the archiving as a hub for microarray data deposit and retrieval. Gene expression profiles of brain tissue in patients with sCJD were compared with those in normal controls to diagnose the differentially expressed genes (DEGs). Selected DEGs were screened by pathway enrichment and gene ontology (GO) enrichment analysis. Subsequently, PPI network construction, module analysis, target genes - miRNA regulatory network construction and target genes - TF regulatory network construction were performed. Finally validation of hub genes was carried out. By using various bioinformatics tools, we may take a further insight of SCJD at molecular level and explore the potential diagnostic and prognostics biomarkers for the therapeutic strategies of sCJD.

\section{Materials and methods}

\section{Microarray data}


medRxiv preprint doi: https://doi.org/10.1101/2020.12.21.20248688; this version posted December 24, 2020. The copyright holder for this preprint (which was not certified by peer review) is the author/funder, who has granted medRxiv a license to display the preprint in perpetuity. All rights reserved. No reuse allowed without permission.

The gene expression profile dataset GSE124571 [13] was downloaded from the GEO database (www.ncbi.nlm.nih.gov/geo/) [14]. The platform for GSE124571 is GPL14951, [Human] Illumina HumanHT-12 WG-DASL V4.0 R2 expression beadchip. The platform files and raw data were downloaded as TXT files. Only data from sCJD and normal control samples were extracted and further analyzed.

\section{Data Preprocessing and DEG Screening}

The R software package beadarray was used to preprocess the downloaded original TXT data. This process involved background adjustment, normalization and expression calculation. Probes not matching any known genes were deleted, and the mean was determined, when numerous probes were balanced to the same gene. The probe ID was converted into gene symbol and saved in a TXT file. The DEGs of SCJD and normal control samples were identified using the $\mathrm{R}$ bioconductor package limma [15]. Genes with an adjusted $p$ value $<0.05,|\log F C|>0.856$ for up regulated genes and $|\log \mathrm{FC}|>-0.84$ for down regulated genes were considered DEGs.

\section{Pathway enrichment analysis of DEGs}

To analyze the identified DEGs at the functional level, BIOCYC (https://biocyc.org/) [16], Kyoto Encyclopedia of Genes and Genomes (KEGG) (http://www.genome.jp/kegg/pathway.html) [17], Pathway Interaction Database (PID) (https://wiki.nci.nih.gov/pages/viewpage.action?pageId=315491760) [18], REACTOME (https://reactome.org/) [19], GenMAPP (http://www.genmapp.org/) [20], MSigDB $\quad$ C2 $\quad$ BIOCARTA (v6.0) (http://software.broadinstitute.org/gsea/msigdb/collections.jsp) [21], PantherDB (http://www.pantherdb.org/) [22], Pathway Ontology (http://www.obofoundry.org/ontology/pw.html) [23] and Small Molecule Pathway Database (SMPDB) (http://smpdb.ca/) [24] pathway analysis were performed using ToppGene (https://toppgene.cchmc.org/enrichment.jsp) [25]. ToppGene is an updated web server which provides a comprehensive set of functional annotation tools for researchers to understand biological meanings behind a large list of genes. In this current study, we analyzed the candidate DEGs that were importantly up and down regulated, and $\mathrm{P}<0.05$ was set as the threshold value.

Gene ontology (GO) enrichment analysis of DEGs 
medRxiv preprint doi: https://doi.org/10.1101/2020.12.21.20248688; this version posted December 24, 2020. The copyright holder for this preprint (which was not certified by peer review) is the author/funder, who has granted medRxiv a license to display the preprint in perpetuity. All rights reserved. No reuse allowed without permission.

GO enrichment analysis (http://www.geneontology.org/) [26] is progressively tested for functional studies of large-scale genomic or transcriptomic data, which constitute three separate ontologies such as biological process (BP), molecular function (MF), and cellular component (CC). The usual up regulated and down regulated genes were analyzed using ToppGene (https://toppgene.cchmc.org/enrichment.jsp) [25], an online program that provides a complete set of function annotation tools for investigators to understand the biological meaning lists of genes. GO enrichment an analysis was performed using ToppGene. $\mathrm{P}<0.05$ was considered to indicate statistically significant difference.

\section{Protein-protein interaction network (PPI) and modular analysis}

To study the interactive relationships among the DEGs, a PPI network of up and down regulated genes were constructed using the IntAct Molecular Interaction Database (https://www.ebi.ac.uk/intact/) [27], which integrates different PPI databases such as Molecular INTeraction Database (MINT, https://mint.bio.uniroma2.it/) [28], UniProt (https://www.uniprot.org/) [29], Interologous interaction database (I2D, http://ophid.utoronto.ca/ophidv2.204/) [30], InnateDB (https://www.innatedb.com/) [31], MatrixDB (http://matrixdb.univlyon1.fr/) [32] and The International Molecular Exchange Consortium (IMEx, http://www.imexconsortium.org/) [33]. Then, the PPI network was visualized using the Cytoscape software (http://www.cytoscape.org) [34]. In this network, each node is a DEG (up or down regulated genes), and the connections between nodes represent the interactions between these genes. PPI network topological properties nodes such as node degree [35], betweenness [36], stress [37], closeness [38] and clustering coefficient [39] were calculated. Modular analysis was conducted with the Molecular Complex Detection PEWCC1 [40] (version 1.3) app of Cytoscape software, and an PEWCC1 score > 2 was set as the cut-off criterion.

\section{Construction of target genes - miRNA regulatory network}

miRNet (https://www.mirnet.ca/) [41] is a comprehensive database of miRNAs, which hosts predicted as well as validated miRNA binding sites. miRNet compares the identified miRNA binding sites with the results obtained from ten established target gene - miRNA prediction databases such as TarBase (http://diana.imis.athena-innovation.gr/DianaTools/index.php?r=tarbase/index) 
medRxiv preprint doi: https://doi.org/10.1101/2020.12.21.20248688; this version posted December 24, 2020. The copyright holder for this

[42], miRTarBase (http://mirtarbase.mbc.nctu.edu.tw/php/download.php) [43], miRecords (http://miRecords.umn.edu/miRecords) [44], miR2Disease (http://www.mir2disease.org/) [45], HMDD (http://www.cuilab.cn/hmdd) [46], PhenomiR (http://mips.helmholtz-muenchen.de/phenomir/) [47], SM2miR (http://bioinfo.hrbmu.edu.cn/SM2miR/) [48], PharmacomiR (http://www.pharmaco-mir.org/) [49], EpimiR (http://bioinfo.hrbmu.edu.cn/EpimiR/) and starBase (http://starbase.sysu.edu.cn/) [51]. The target gene - miRNA pairs were visualized by Cytoscape software (http://www.cytoscape.org) [34].

\section{Construction of target genes - TF regulatory network}

NetworkAnalyst (https://www.networkanalyst.ca/) [52] is a web based tool which can search transcription factors for the input up and down regulated genes as well as assess the effect of a transcription factor on the expression of the target genes. In this study, the transcription factors of the target genes were predicted from NetworkAnalyst database which ingrates TF database ChEA (http://amp.pharm.mssm.edu/lib/chea.jsp) [53] and target genes - TF regulatory network was constructed and visualized by Cytoscape software(http://www.cytoscape.org) [34].

\section{Validation of hub genes}

Receiver operating characteristic (ROC) curves were used to analyze the potential clinical significance of these hub genes as molecular prognostic markers in SCJD. Based on the obtained optimal prognostic gene biomarkers for SCJD, we established the GLM (generalized linear models) was a managed learning model by using the pROC package [54] in R software. The prognostic ability of this models was accessed by obtaining the area under a ROC curve (AUC), accuracy, sensitivity and specificity. $\mathrm{P}<0.05$ was considered to indicate a statistically significant difference.

\section{Results}

\section{Identification of DEGs in sCJD}

After gene expression profile data processing and standardization, we screened DEGs in GSE124571 dataset using integrated bioinformatics analysis and the 
medRxiv preprint doi: https://doi.org/10.1101/2020.12.21.20248688; this version posted December 24, 2020. The copyright holder for this

results are shown in Fig. 1A and Fig. 1B. The limma package of the R software identified 891 DEGs (Table 1), with the cutoff standard of $\mathrm{P}$ value $<0.05,|\log \mathrm{FC}|$ $>0.856$ for up regulated genes and $|\operatorname{logFC}|>-0.84$ for down regulated genes; 448 of these DEGs presented significant up regulated, and the remaining 443 down regulated. Fig. 2 presents the volcano plot of DEGs for data set. A cluster heatmap developed with $\mathrm{R}$ software showed the distribution of all up and down regulated genes (Fig. 3 and Fig. 4).

\section{Pathway enrichment analysis of DEGs}

Pathway enrichment analysis of DEGs was conducted with ToppGene, and the results are given in Table 2 and Table 3. The signaling pathways of up regulated were mainly enriched in glutamine degradation/glutamate biosynthesis, asparagine biosynthesis, GABAergic synapse, synaptic vesicle cycle, effects of Botulinum toxin, role of calcineurin-dependent NFAT signaling in lymphocytes, transmission across chemical synapses, neuronal system, type III secretion system, ATP synthesis, regulation of PGC-1a, bioactive peptide induced signaling pathway, muscarinic acetylcholine receptor 1 and 3 signaling pathway, oxytocin receptor mediated signaling pathway, glutamate metabolic, insulin secretion pathway, cimetidine pathway and malate-aspartate shuttle, whereas down regulated were mainly enriched in melatonin degradation II, proline degradation, leishmaniasis, phagosome, validated targets of C-MYC transcriptional repression, CXCR4mediated signaling events, neutrophil degranulation, cytokine signaling in immune system, inositol phosphate metabolism, monocyte and its surface molecules, cystic fibrosis transmembrane conductance regulator and beta 2 adrenergic receptor pathway, T cell activation, 5-hydroxytryptamine degredation, integrin signaling, altered lipoprotein metabolic, lactic acidemia and lysosomal acid lipase deficiency (Wolman Disease).

\section{Gene ontology (GO) enrichment analysis of DEGs}

Gene ontology (GO) enrichment analysis of DEGs was performed with ToppGene, and, up and down regulated genes were classified into three functional groups such as biological processes (BP), molecular functions (MF) and cell compositions (CC). The results of Gene ontology (GO) enrichment analysis are given in Table 4 and Table 5. In the BP group, the up regulated genes were mainly enriched in 
medRxiv preprint doi: https://doi.org/10.1101/2020.12.21.20248688; this version posted December 24, 2020. The copyright holder for this

chemical synaptic transmission and neuron projection development, and the down regulated genes were mainly enriched in the regulation of immune system process and cell activation. In the $\mathrm{CC}$ group, the up regulated genes were mainly enriched in neuron part and synapse, and the down regulated genes were mainly enriched in the membrane region and cell surface. In the MF group, the up regulated genes were mainly enriched in cytoskeletal protein binding and molecular function regulator, and the down regulated genes were mainly enriched in the enzyme binding and molecular transducer activity.

\section{Protein-protein interaction network (PPI) and modular analysis}

The potential relationships among these DEGs (up and down regulated genes) at protein levels were predicted based on the IntAct Molecular Interaction database. As shown in Fig. 5, the establishment of the PPI network for up regulated genes identified 3664 nodes and 6054 edges. The top hub genes (GABARAPL1, YWHAZ, SSX2IP, YWHAH, UBE2N, CALM3, TUBB3, NME5, CAPRIN2, BEX5, SV2B and KCNIP4) were identified based on their connectivity degree, betweenness centrality, stress centrality, closeness centrality and clustering coefficient and are listed in Table 6. Scatter plot along with statistical results for node degree, betweenness centrality, stress centrality, closeness centrality and clustering coefficient are shown in Fig. 6A - 6E. Pathways and GO enrichment analysis revealed that these hub genes were markedly enriched in GABAergic synapse, role of calcineurin-dependent NFAT signaling in lymphocytes, cell projection organization, chemical synaptic transmission, enzyme binding, oxytocin signaling pathway, Huntington disease, cell morphogenesis, signaling receptor binding, cell-cell signaling and ion transport. As shown in Fig. 7, the establishment of the PPI network for down regulated genes identified 3095 nodes and 4747 edges. The top hub genes (HSPB1, HDAC1, CDKN1A, TNFRSF1A, FKBP5, BCL6, MYL12A, SPTBN1, SFMBT2, NUPR1, TRIM47, RAPGEF3 and MS4A7) were identified based on their connectivity degree, betweenness centrality, stress centrality, closeness centrality and clustering coefficient and are listed in Table 6. Scatter plot along with statistical results for node degree, betweenness centrality, stress centrality, closeness centrality and clustering coefficient are shown in Fig. 8A - 8E. Pathways and GO enrichment analysis revealed that these hub genes were markedly enriched in cell activation, validated targets of C-MYC transcriptional repression, HTLV-I infection, defense response, direct p53 effectors, regulation of 
medRxiv preprint doi: https://doi.org/10.1101/2020.12.21.20248688; this version posted December 24, 2020. The copyright holder for this

actin cytoskeleton, neutrophil degranulation, regulation of cell proliferation and hemostasis,

To explore the significance of the molecules of the sCJD related PPI network, the module analysis was performed by PEWCC1. The four most significant modules of the PPI network for up regulated genes were shown in Fig 9, in which GABARAPL1, YWHAZ, YWHAH, EPB41L3, KLC1, CYFIP2, NCKAP1, MAGED1, RBFOX1, RBFOX2, SSX2IP, TUBB2A, USP11, PFN2, HPRT1, NCOA and ENO2 were the top hub genes. Pathway and GO enrichment analyses of these hub genes associated in these module were performed using ToppGene. Results showed that the genes in these significant modules were predominantly enriched for terms associated with GABAergic synapse, neurotrophic factor-mediated Trk receptor signaling, Huntington disease, superpathway of purine nucleotide salvage, cell-cell signaling, neuron projection development, cell projection organization and chemical synaptic transmission. The four most significant modules of the PPI network for down regulated genes were shown in Fig 10, in which PTPN6, CD37, EZR, MSN, SLC9A3R1, CD44, CEBPA, CEBPB, CEBPD, HSPB1, LYN, HSPB8, BAG3 and CRYAB were the top hub genes. Pathway and GO enrichment analyses of these hub genes associated in these module were performed using ToppGene. Results showed that the genes in these significant modules were predominantly enriched for terms associated with leishmaniasis, regulation of actin cytoskeleton, validated targets of C-MYC transcriptional repression, regulation of immune system process, biological adhesion, cell activation, cell adhesion and identical protein binding.

\section{Construction of target gene - miRNA regulatory network}

The target gene - miRNA regulatory network for up regulated genes is shown in Fig. 11. Top five up regulated target genes such as TUBB2A interacts with 183 miRNAs (ex, hsa-mir-5681a), YWHAZ interacts with 156 miRNAs (ex, hsa-mir3189-5p), MAP3K9 interacts with 121 miRNAs (ex, hsa-mir-6791-3p), PGM2L1 interacts with 117 miRNAs (ex, hsa-mir-6764-3p) and ALDOA interacts with 112 miRNAs (ex, hsa-mir-4476) are listed in Table 7. Pathways and GO enrichment analysis revealed that these target genes were markedly enriched in Huntington disease, role of calcineurin-dependent NFAT signaling in lymphocytes, purine nucleotide binding and identical protein binding. Similarly, target gene - miRNA 
medRxiv preprint doi: https://doi.org/10.1101/2020.12.21.20248688; this version posted December 24, 2020. The copyright holder for this

regulatory network for down regulated genes is shown in Fig. 12. Top five down regulated target genes such as CCND1 interacts with 197 miRNAs (ex, hsa-mir3973), MKNK2 interacts with 153 miRNAs (ex, hsa-mir-6515-5p), CDKN1A interacts with 131 miRNAs (ex, hsa-mir-6886-3p), SYNJ2BP interacts with 120 miRNAs (ex, hsa-mir-4659a-3p) and BRI3BP interacts with 119 miRNAs (ex, hsamir-3689b-3p) are listed in Table 7. Pathways and GO enrichment analysis revealed that these target genes were markedly enriched in HTLV-I infection, regulation of immune system process and locomotion.

\section{Construction of target genes - TF regulatory network}

The target gene - TF regulatory network for up regulated genes is shown in Fig.13. Top five up regulated target genes such as CDK14 interacts with 235 TFs (ex, SOX2), YWHAH interacts with 193 TFs (ex, MYC), SYN2 interacts with 182 TFs (ex, REST), SYT1 interacts with 179 TFs (ex, SUZ12) and MICAL2 interacts with 169 TFs (ex, AR) are listed in Table 8. Pathways and GO enrichment analysis revealed that these target genes were markedly enriched in purine nucleotide binding, role of calcineurin-dependent NFAT signaling in lymphocytes, transmission across chemical synapses, synaptic vesicle cycle and cytoskeletal protein binding. Similarly, target gene - miRNA regulatory network for down regulated genes is shown in Fig.14. Top five down regulated target genes such as HSPA1A interacts with 224 TFs (ex, SOX2), CD300A interacts with 212 TFs (ex, SPI1), HLA-DOA interacts with 197 TFs (ex, EGR1), TST interacts with 184 TFs (ex, HNF4A) and HSPB1 interacts with 179 TFs (ex, MYC) are listed in Table 8. Pathways and GO enrichment analysis revealed that these target genes were markedly enriched in toxoplasmosis, neutrophil degranulation, extracellular space and cell activation.

\section{Validation of hub genes}

Based on these hub genes between sCJD and normal control, the GLM model was established. AUC values of YWHAZ, GABARAPL1, SSX2IP, YWHAH, UBE2N, HSPB1, HDAC1, CDKN1A, TNFRSF1A and FKBP5 were 0.891, 0.909, $0.873,0.809,0.927,0.973,0.936,0.800,0.918$ and 0.945 suggesting that these ten hub genes had high sensitivity and specificity for SCJD prognosis. The ROC results are displayed in Fig. 15. These results indicated that YWHAZ, GABARAPL1, 
medRxiv preprint doi: https://doi.org/10.1101/2020.12.21.20248688; this version posted December 24, 2020. The copyright holder for this

SSX2IP, YWHAH, UBE2N, HSPB1, HDAC1, CDKN1A, TNFRSF1A and FKBP5 may be used as biomarkers for the prognosis of sCJD.

\section{Discussion}

With the development of microarray and high-throughput sequencing technology in current years, integrated bioinformatics methods have been broadly applied in discovering new biomarkers correlated with the diagnosis, prognosis, and treatment of numerous brain diseases [55-56]. sCJD is an prion disease which has brought a heavy burden to European, Australia, and Canadian countries [57]. Understanding the molecular mechanism of sCJD is of essential importance for prognosis, diagnosis and treatment. It has been extensively used to predict therapeutic targets for sCJD since high-throughput sequencing can implement expression levels of genes in human genome simultaneously. The aim of this study was to diagnose several key genes and pathways with similar action highly expressed in SCJD compared to normal controls and discover their potential mechanisms. In the current study, we extract the gene expression profile of GSE124571 downloaded from GEO database and identify 448 up regulated and 443 down regulated genes between RA and normal control using bioinformatics analysis. Less expression of VAMP2 was associated with development of dementia [58], but decrease expression of this gene may be responsible for progression of sCJD. CIRBP (cold inducible RNA binding protein) was linked with neuroinflammation in cerebral ischemia [59], but this gene may be liable for neuroinflammation in SCJD. CRYM (crystallin mu) was involved in progression of Huntington's disease [60], but this gene may be liable for advancement of sCJD. DOCK3 was involved in growth of hyperactivity disorder [61], but this gene may be associated with progression of sCJD. Genes such as CYFIP2 [62] PC (pyruvate carboxylase) [63] and PLOD1 [64] were responsible for advancement of Alzheimer's disease, but these genes may be involved in pathogenesis of sCJD. PLOD3 was liable for advancement of brain cancer [65], but this gene may be associated with growth of sCJD. High expression of CRYAB (crystallin alpha B) [66] and DNAJB6 [67] was important for development of Parkinson's disease, but this gene may be associated with development of SCJD.

In pathway enrichment analysis, up regulated genes were enriched in various pathways. Enriched genes such as GLS2 [68], SLC12A5 [69], GAD1 [70], GAD2 
medRxiv preprint doi: https://doi.org/10.1101/2020.12.21.20248688; this version posted December 24, 2020. The copyright holder for this preprint (which was not certified by peer review) is the author/funder, who has granted medRxiv a license to display the preprint in perpetuity. All rights reserved. No reuse allowed without permission.

[71], STXBP1 [72], NPTN (neuroplastin) [73] and PPP3CB [74] were associated with development of schizophrenia, but these genes may be important for pathogenesis of sCJD. GLS (glutaminase) was linked with neuroinflammation in brain diseases [75], but this gene may be involved with neuroinflammation in sCJD. Alteration in GNB5 was responsible for advancement of hyperactivity disorder [76], but mutation in this gene may be identified with development of sCJD. Enriched genes such as PRKCB (protein kinase C beta) [77], SNAP25 [78], CALM3 [79], ATP6V0C [80] and SST (somatostatin) [81] were important for pathogenesis of Alzheimer's disease, but this gene may be linked with progression of sCJD. Enriched genes such as PRKCG (protein kinase C gamma), [82] ITPR1 [83] and VAMP1 [84] were involved in advancement of spinocerebellar ataxia, but these genes may be important for advancement of sCJD. Modification in GABRA1 [85], GABRB3 [86] and GABRG2 [87] were identified with progression of epilepsy, but alteration in these genes may be linked with pathogenesis of sCJD. Mutation in GNAO1 was responsible for progression of movement disorder [88], but Alteration in this gene may associated with development of SCJD. Enriched genes such as STX1A [89], LIN7B [90] and CACNB2 [91] were involved in development of autism, but these genes may be liable for advancement of sCJD. Enriched genes such as NEFL (neurofilament light) [92] and YWHAH (tyrosine 3monooxygenase/tryptophan 5-monooxygenase activation protein eta) [93] were responsible for development of sCJD. ATP6V1B2 was associated with progression of depression, but this gene may be identified with advancement of sCJD [94]. CHRM1 was associated with pathogenesis of Huntington's disease [95], but this gene may be liable for advancement of sCJD. Polymorphism in CCKBR (cholecystokinin B receptor) was liable for advancement of Parkinson's disease [96], this polymorphic gene may be associated with progression of sCJD. Our study found that GNG2, ADCY1, GABARAPL1, GABBR2, SLC32A1, GABRD (gamma-aminobutyric acid type A receptor delta subunit), SLC38A1, GNG3, NSF (N-ethylmaleimide sensitive factor, vesicle fusing ATPase), SYT1, NCALD (neurocalcin delta), CACNG3, CPLX1, KCNJ4, KCNJ12, SYN1, SYN2, CAMK2B， CAMK2G， EPB41L1，ATP6V1A， ATP6V1E1，ATP6V1G2, CAMK1G, PRKCE (protein kinase C epsilon) and GOT1 are up regulated in SCJD and has potential as a novel diagnostic and prognostic biomarker, and therapeutic target. Down regulated genes were enriched in various pathways. Enriched genes such as MAOA (monoamine oxidase A) [97], TGFB3 [98], CEBPD (CCAAT 
medRxiv preprint doi: https://doi.org/10.1101/2020.12.21.20248688; this version posted December 24, 2020. The copyright holder for this

enhancer binding protein delta) [99], NDRG2 [100], SLC11A1 [101], HDAC1 [102], TYROBP (TYRO protein tyrosine kinase binding protein) [103], ALOX5 [104], DOCK2 [105], OLR1 [106], CD14 [107], CD44 [108], CD68 [109], ITPKB (inositol-trisphosphate 3-kinase B) [110], WAS (Wiskott-Aldrich syndrome) [111], CD86 [112] and CD74 [113] were linked with development of Alzheimer's disease, but these genes may be involved in progression of sCJD. HLA-DPA1 was involved in progression of acute disseminated encephalomyelitis [114], but this gene may be important for development of sCJD. Enriched genes such as HLADQA1 [115] and IRF8 [116] were associated with advancement of multiple sclerosis, but these genes may be responsible for pathogenesis of sCJD. Enriched genes such as IFNGR1 [117], PRDX6 [118] and LAMP2 [119] were identified with progression of Parkinson's disease, but these genes may be linked with advancement of sCJD. NDRG1 was liable for progression of Charcot-Marie-Tooth disease [120], but this gene may be identified with development of sCJD. ITGB4 was liable for progression of bipolar disorder [121], but this gene may be important for advancement of sCJD. SERPINA3 was involved in advancement of sCJD [122]. CLEC5A was important for development of brain cancer [123], but this gene be linked with pathogenesis of SCJD. HSPA1A was responsible for progression of schizophrenia [124], but this gene identified with pathogenesis of sCJD. NPC2 was associated with growth of Niemann-Pick C2 disease [125], but this gene may be important for pathogenesis of sCJD. RNASET2 was linked with progression of cystic leukoencephalopathy [126], but this gene may be liable for advancement of sCJD. Our study found that HLA-DMA (major histocompatibility complex, class II, DM alpha), HLA-DMB (major histocompatibility complex, class II, DM beta), HLA-DOA (major histocompatibility complex, class II, DO alpha), HLA-DRA (major histocompatibility complex, class II, DR alpha), FCGR1A, CYBA (cytochrome b-245 alpha chain), ITGB1, ITGB2, PTPN6, CDKN1A, CEBPA (CCAAT enhancer binding protein alpha), CCND1, ATP8B4, CD300A, CTSC (cathepsin C), TMC6, SERPINB6, FCER1G, FGR (FGR proto-oncogene, Src family tyrosine kinase), SLC2A5, RHOG (ras homolog family member G), PRCP (prolylcarboxypeptidase), PYCARD (PYD and CARD domain containing), VAMP8, CTSH (cathepsin H), ITGAX (integrin subunit alpha X), ADA2, TMBIM1, BST2, LILRB3, RAB31, STOM (stomatin), PLCD1, INPPL1, CSK (Cterminal Src kinase), APBB1IP, PARVG (parvin gamma) and ITGB5 are down 
medRxiv preprint doi: https://doi.org/10.1101/2020.12.21.20248688; this version posted December 24, 2020. The copyright holder for this preprint (which was not certified by peer review) is the author/funder, who has granted medRxiv a license to display the preprint in perpetuity. All rights reserved. No reuse allowed without permission.

regulated in SCJD and has potential as a novel diagnostic and prognostic biomarker, and therapeutic target.

In $\mathrm{GO}$ enrichment analysis, up regulated genes were enriched in all $\mathrm{GO}$ categories. Enriched genes such as RTN3 [127], VGF (VGF nerve growth factor inducible) [128], SNCA (synuclein alpha) [129], SYP (synaptophysin) [130], NPTX1 [131], NRGN (neurogranin) [132], EGR1 [133], CDK5 [134], MAP1B [135], UCHL1 [136], VPS35 [137], CNTNAP2 [138], KLC1 [139], FZD3 [140], CCK (cholecystokinin) [141], EPHA4 [142], TPM1 [143] and WASF1 [144] were responsible for progression of Alzheimer's disease, but these genes may be associated in progression of sCJD. PFN2 was liable for development of CharcotMarie-Tooth disease [145], but this gene may be linked with pathogenesis of sCJD. Enriched genes such as ADCYAP1 [146], NRXN1 [147] LRRTM1 [148], BASP1 [72] and DCLK1 [149] were involved in progression of schizophrenia, but these genes may be responsible for advancement of sCJD. Enriched genes such as SCN1B [150], FGF12 [151], NAPB (NSF attachment protein beta) [152], DNM1 [153] and AP3B2 [154] were important in advancement of epilepsy, but these genes may be identified with progression of sCJD. Enriched genes such as SNCB (synuclein beta) [155], RIT2 [156], TUBB3 [157], PVALB (parvalbumin) [158] and ELAVL4 [159] were involved in progression of Parkinson's disease, but these genes may be linked with development of sCJD. Enriched genes such as CADPS2 [160], ATP2B2 [161] and ENO2 [162] were important for progression of autism, but these genes may be identified with pathogenesis of sCJD. TAC1 was responsible for advancement of multiple sclerosis [163], but this gene may be associated with progression of sCJD. Enriched genes such NPY (neuropeptide Y) [164], MAP2 [165], APP (amyloid beta precursor protein) [166], STMN2 [167] and PRNP (prion protein) [168] were important for advancement of sCJD. PACSIN1 was liable for advancement of Huntington's disease [169], but this gene may be involved in progression of sCJD. Our study found that LRFN5, SCN2B, SYT13, AMPH (amphiphysin), ICA1, ADGRL1, PNKD (PNKD, MBL domain containing), SLC8A2, ATP2A2, CLSTN3, CPLX2, PTPRN2, SV2B, GLRB (glycine receptor beta), KALRN (kalirinRhoGEF kinase), SYNGR1, PAK1, PCP4, PDE1A, MAP4, CYGB (cytoglobin), ACOT7, HPCA (hippocalcin), AP1S1, SCAMP5, NGEF (neuronal guanine nucleotide exchange factor), OLFM1, FLRT3, FKBP1A, SVOP (SV2 related protein), SLC9A6, CAP2, MAP2K4, ATCAY 
medRxiv preprint doi: https://doi.org/10.1101/2020.12.21.20248688; this version posted December 24, 2020. The copyright holder for this preprint (which was not certified by peer review) is the author/funder, who has granted medRxiv a license to display the preprint in perpetuity. All rights reserved. No reuse allowed without permission.

(ATCAY, caytaxin), CNTNAP1, ENC1, MYRIP (myosin VIIA and Rab interacting protein), MAGEE1, PPP1R2, ERC2, KCNIP4, ATP1A1, TBR1, NRSN1, MAPK9, MAP2K1，ATP2B1，ARHGAP32， NMNAT2，FRMPD4, SLC6A17, KCTD8, RAP1GAP2, LAMP5, EPB41L3, SLC30A3, NEFM (neurofilament medium), NEFH (neurofilament heavy), NELL2, PTPRN (protein tyrosine phosphatase, receptor type N), ARHGAP44, KIF3C, SLC17A6, GPM6A, DPP6, DMXL2, WDR7, RTN4, STMN1, RGS7, OPA1, SYNGR3, ALDOA (aldolase, fructose-bisphosphate A), BAIAP2L2, GAS7, SYBU (syntabulin), RAB6B, MICAL2, ABLIM2, REEP1, NME1, CDK5R2, DYNC1I1, PTPRT (protein tyrosine phosphatase, receptor type T) and CORO6 are up regulated in sCJD and has potential as a novel diagnostic and prognostic biomarker, and therapeutic target. Down regulated genes were enriched in all GO categories. Enriched genes such as HMOX1 [170], RGCC (regulator of cell cycle) [171], CSF1R [172], HTRA1 [173], BCL6 [174], SYK (spleen associated tyrosine kinase) [175], C1QB [176], ADAM17 [177], PLA2G7 [178], TLR5 [179], PDE9A [180], ITSN1 [181], GJA1 [182], FERMT2 [183], NTRK2 [184], TNFRSF1A [185], S100A1 [186], ALOX5AP [104], APOC2 [187], DIO2 [188] and GSTM3 [189] were responsible for progression of Alzheimer's disease, but these genes may be liable in progression of sCJD. Enriched genes such as AIF1 [190], ABCA1 [191], AQP4 [192] and GFAP (glial fibrillary acidic protein) [193] were important for pathogenesis of sCJD. Enriched genes such as FGFR3 [194], VEGFB (vascular endothelial growth factor B) [195], GPER1 [196], WASF2 [197], ADRB2 [198], ATXN3 [199], SGK1 [200] and TIMP1 [201] were identified with growth of Parkinson's disease, but these genes may be linked with progression of sCJD. SLC1A3 was associated with development of hyperactivity disorder [202], but this gene may be liable for progression of sCJD. MEGF10 was involved in progression of schizophrenia [203], but this gene may be liable for advancement of sCJD. Our study found that CMTM3, CEBPB (CCAAT enhancer binding protein beta), PDK4, VSIG4, RFTN2, LY96, VAMP3, SASH3, FCGRT (Fc fragment of IgG receptor and transporter), MERTK (MER proto-oncogene, tyrosine kinase), CHST3, EZR (ezrin), LAT2, MSN (moesin), SLC7A2, CSF3R SCIN (scinderin), GPRC5B, MYOM1, TNFSF14, RASSF2, MYO10, AXL (AXL receptor tyrosine kinase), LILRB1, VSIR (V-set immunoregulatory receptor), HCST (hematopoietic cell signal transducer), STAT5A, MAPKAPK3, C1QA, C1QC, UNC93B1, TAP1, RAC2, RARRES2, GPX1, LGALS9, HAVCR2, EBI3, CD37, LYN (LYN proto- 
medRxiv preprint doi: https://doi.org/10.1101/2020.12.21.20248688; this version posted December 24, 2020. The copyright holder for this

oncogene, Src family tyrosine kinase), TRIB1, HCK (HCK proto-oncogene, Src family tyrosine kinase), HCLS1, HERC5, CYBRD1, DHRS3, SLCO2B1, ADGRV1, FZD9, SLC9A3R1, RAPGEF3, AHNAK (AHNAK nucleoprotein), ANTXR1, PLEK (pleckstrin), CNGB1, SDC4, HSPB1, PDPN (podoplanin), NECAP2, ATP1B2, PIEZO1, SPTBN1, DAB2, MFRP (membrane frizzled-related protein), PTH1R, HEPH (hephaestin), KANK1, KCNMA1, GNA12, DDR1, GRIN2C, LCP1, SLC7A7, RHOQ (ras homolog family member Q), SYTL4, LRP4, EPHX1, CARHSP1, ADD3, SRGAP1, FOXO4, WFS1, RHOBTB3, PPP1R3C, ARHGDIB (Rho GDP dissociation inhibitor beta), RANBP3L, ZFHX3, MAPK4, NEK6, RAB3IL1, NACC2 and FARP1 are down regulated in SCJD and has potential as a novel diagnostic and prognostic biomarker, and therapeutic target.

Up regulated hub genes were identified from PPI network for up regulated genes. YWHAZ was responsible for progression of schizophrenia [204], but this gene may liable for advancement of sCJD. Our study found that SSX2IP, UBE2N, NME5, CAPRIN2 and BEX5 are up regulated in SCJD and has potential as a novel diagnostic and prognostic biomarker, and therapeutic target. Down regulated hub genes were identified from PPI network for down regulated genes. Our study found that FKBP5, MYL12A, SFMBT2, NUPR1, TRIM47 and MS4A7 are down regulated in SCJD and has potential as a novel diagnostic and prognostic biomarker, and therapeutic target.

In module analysis, up regulated hub genes showing the highest node degree in all four significant modules. NCKAP1 was linked with development of Alzheimer's disease [205], but this gene may be involved in progression of sCJD. RBFOX1 was important for progression autism [206], but this gene may be identified with pathogenesis of sCJD. Our study found that MAGED1, RBFOX2, TUBB2A, USP11, HPRT1 and NCOA7 are up regulated in SCJD and has potential as a novel diagnostic and prognostic biomarker, and therapeutic target. Similarly, down regulated hub genes showing the highest node degree in all four significant modules. Genes such as HSPB8 [207] and BAG3 [208] were responsible for advancement of Alzheimer's disease, but these genes may be important for progression of sCJD. 
medRxiv preprint doi: https://doi.org/10.1101/2020.12.21.20248688; this version posted December 24, 2020. The copyright holder for this

In target gene - miRNA network, up regulated target genes showing the highest number of integration with miRNAs. Our study found that MAP3K9 and PGM2L1 are up regulated in SCJD and has potential as a novel diagnostic and prognostic biomarker, and therapeutic target. Similarly, down regulated target genes showing the highest number of integration with miRNAs. Our study found that MKNK2, SYNJ2BP and BRI3BP are down regulated in sCJD and has potential as a novel diagnostic and prognostic biomarker, and therapeutic target

In target gene - TF network, up regulated target genes showing the highest number of integration with TFs. Our study found that CDK14 is up regulated in sCJD and has potential as a novel diagnostic and prognostic biomarker, and therapeutic target. Similarly, down regulated target genes showing the highest number of integration with TFs. Our study found that TST is down regulated in SCJD and has potential as a novel diagnostic and prognostic biomarker, and therapeutic target.

In conclusion, although the current study had certain conditions, including the limited number of cases and the absence of validation in clinical samples, the current analysis diagnosed distinct important genes and pathways closely linked with SCJD, which may contribute to the current knowledge of the complex molecular mechanisms of sCDJ. Of note, the current results credential acceptance by further examination.

\section{Acknowledgement}

We thank Piero Parchi, University of Bologna, DIMES, Neuropathology, Bologna, Italy, very much, the authors who deposited their microarray dataset, GSE124571, into the public GEO database.

\section{Availability of data and materials}

The datasets supporting the conclusions of this article are available in the GEO (Gene Expression Omnibus) (https://www.ncbi.nlm.nih.gov/geo/) repository. [(GSE124571) (https://www.ncbi.nlm.nih.gov/geo/query/acc.cgi?acc= GSE124571)]

\section{Consent for publication}


Not applicable.

\section{Competing interests}

The authors declare that they have no competing interests.

\section{Conflict of interest}

The authors declare that they have no conflict of interest.

\section{Ethical approval}

This article does not contain any studies with human participants or animals performed by any of the authors.

\section{Informed consent}

No informed consent because this study does not contain human or animals participants.

\section{Author Contributions}

B. V. - Writing original draft, and review and editing

C. V. - Software and investigation

I. K. - Supervision and resources

\section{Authors}

Basavaraj Vastrad

Chanabasayya Vastrad

Iranna Kotturshetti
ORCID ID: 0000-0003-2202-7637

ORCID ID: 0000-0003-3615-4450

ORCID ID: 0000-0003-1988-7345

\section{References}

Hill AF, Butterworth RJ, Joiner S, Jackson G, Rossor MN, Thomas DJ, Frosh A, Tolley N, Bell JE, Spencer $M$ et al. Investigation of variant Creutzfeldt-Jakob disease and other human prion diseases with tonsil biopsy samples. Lancet. 1999;353(9148):183-189. doi:10.1016/s0140-6736(98)12075-5 
medRxiv preprint doi: https://doi.org/10.1101/2020.12.21.20248688; this version posted December 24, 2020. The copyright holder for this preprint (which was not certified by peer review) is the author/funder, who has granted medRxiv a license to display the preprint in perpetuity. All rights reserved. No reuse allowed without permission.

Palmer MS, Dryden AJ, Hughes JT, Collinge J. Homozygous prion protein genotype predisposes to sporadic Creutzfeldt-Jakob disease. Nature. 1991;352(6333):340-342. doi:10.1038/352340a0

Paulson HL. Protein fate in neurodegenerative proteinopathies: polyglutamine diseases join the (mis)fold. Am J Hum Genet. 1999;64(2):339-345. doi:10.1086/302269

Cali I, Castellani R, Yuan J, Al-Shekhlee A, Cohen ML, Xiao X, Moleres FJ, Parchi P, Zou WQ, Gambetti P. Classification of sporadic Creutzfeldt-Jakob disease revisited. Brain. 2006;129(Pt 9):2266-2277. doi:10.1093/brain/awl224

Mead S, Poulter M, Uphill J, Beck J, Whitfield J, Webb TE, Campbell T, Adamson G, Deriziotis P, Tabrizi SJ et al. Genetic risk factors for variant Creutzfeldt-Jakob disease: a genome-wide association study. Lancet Neurol. 2009;8(1):57-66. doi:10.1016/S1474-4422(08)70265-5

López González I, Garcia-Esparcia P, Llorens F, Ferrer I. Genetic and Transcriptomic Profiles of Inflammation in Neurodegenerative Diseases: Alzheimer, Parkinson, Creutzfeldt-Jakob and Tauopathies. Int J Mol Sci. 2016;17(2):206. doi:10.3390/ijms17020206

Richardson EP., Masters CL. The nosology of Creutzfeldt-Jakob disease and conditions related to the accumulation of PrPCJD in the nervous system. Brain Pathol. 1995;5(1):33-41.

Spudich S, Mastrianni JA, Wrensch M, Gabizon R, Meiner Z, Kahana I, Rosenmann H, Kahana E, Prusiner SB. Complete penetrance of Creutzfeldt-Jakob disease in Libyan Jews carrying the E200K mutation in the prion protein gene. Mol Med. 1995;1(6):607-613.

Monari L, Chen SG, Brown P, Parchi P, Petersen RB, Mikol J, Gray F, Cortelli P, Montagna P, Ghetti B, et al. Fatal familial insomnia and familial Creutzfeldt-Jakob disease: different prion proteins determined by a DNA polymorphism. Proc Natl Acad Sci U S A. 1994;91(7):2839-2842. doi:10.1073/pnas.91.7.2839 
medRxiv preprint doi: https://doi.org/10.1101/2020.12.21.20248688; this version posted December 24, 2020. The copyright holder for this preprint (which was not certified by peer review) is the author/funder, who has granted medRxiv a license to display the preprint in perpetuity. All rights reserved. No reuse allowed without permission.

He D, Liu L, Wang Y, Sheng M. A Novel Genes Signature Associated with the Progression of Polycystic Ovary Syndrome. Pathol Oncol Res. 2019. doi:10.1007/s12253-019-00676-3

Li C, Zeng X, Yu H, Gu Y, Zhang W. Identification of hub genes with diagnostic values in pancreatic cancer by bioinformatics analyses and supervised learning methods. World J Surg Oncol. 2018;16(1):223. doi:10.1186/s12957-018-1519-y

Haage V, Semtner M, Vidal RO, Hernandez DP, Pong WW, Chen Z, Hambardzumyan D, Magrini V, Ly A, Walker J et al. Comprehensive gene expression meta-analysis identifies signature genes that distinguish microglia from peripheral monocytes/macrophages in health and glioma. Acta Neuropathol Commun. 2019;7(1):20. doi:10.1186/s40478-019-0665-y

Bartoletti-Stella A, Corrado P, Mometto N, Baiardi S, Durrenberger PF, Arzberger T, Reynolds R, Kretzschmar H, Capellari S, Parchi P. Analysis of RNA Expression Profiles Identifies Dysregulated Vesicle Trafficking Pathways in Creutzfeldt-Jakob Disease. Mol Neurobiol. 2019;56(7):5009-5024. doi:10.1007/s12035-018-1421-1

Barrett T, Wilhite SE, Ledoux P, Evangelista C, Kim IF, Tomashevsky M, Marshall KA, Phillippy KH, Sherman PM, Holko M et al. NCBI GEO: archive for functional genomics data sets--update. Nucleic Acids Res. 2013;41(Database issue):D991-D995. doi:10.1093/nar/gks1193

Ritchie ME, Phipson B, Wu D, Hu Y, Law CW, Shi W, Smyth GK. limma powers differential expression analyses for RNA-sequencing and microarray studies. Nucleic Acids Res. 2015;43(7):e47. doi:10.1093/nar/gkv007

Caspi R, Billington R, Ferrer L, Foerster H, Fulcher CA, Keseler IM, Kothari A, Krummenacker M, Latendresse M, Mueller LA et al. The MetaCyc database of metabolic pathways and enzymes and the BioCyc collection of pathway/genome databases. Nucleic Acids Res. 2016;44(D1):D471-D480. doi:10.1093/nar/gkv1164

Kanehisa M, Furumichi M, Tanabe M, Sato Y, Morishima K. KEGG: new perspectives on genomes, pathways, diseases and drugs. Nucleic Acids Res. 2017;45(D1):D353-D361. doi:10.1093/nar/gkw1092 
medRxiv preprint doi: https://doi.org/10.1101/2020.12.21.20248688; this version posted December 24, 2020. The copyright holder for this preprint (which was not certified by peer review) is the author/funder, who has granted medRxiv a license to display the preprint in perpetuity. All rights reserved. No reuse allowed without permission.

Schaefer CF, Anthony K, Krupa S, Buchoff J, Day M, Hannay T, Buetow KH. PID: the Pathway Interaction Database. Nucleic Acids Res. 2009;37(Database issue):D674-D679. doi:10.1093/nar/gkn653

Sidiropoulos K, Viteri G, Sevilla C, Jupe S, Webber M, Orlic-Milacic M, Jassal B, May B, Shamovsky V, Duenas $\mathrm{C}$ et al. Reactome enhanced pathway visualization. Bioinformatics. 2017;33(21):3461-3467. doi:10.1093/bioinformatics/btx441

Dahlquist KD, Salomonis N, Vranizan K, Lawlor SC, Conklin BR. GenMAPP, a new tool for viewing and analyzing microarray data on biological pathways. Nat Genet. 2002;31(1):19-20. doi:10.1038/ng0502-19

Subramanian A, Tamayo P, Mootha VK, Mukherjee S, Ebert BL, Gillette MA, Paulovich A, Pomeroy SL, Golub TR, Lander ES et al. Gene set enrichment analysis: a knowledge-based approach for interpreting genome-wide expression profiles. Proc Natl Acad Sci U S A. 2005;102(43):15545-15550. doi:10.1073/pnas.0506580102

Mi H, Huang X, Muruganujan A, Tang H, Mills C, Kang D, Thomas PD. PANTHER version 11: expanded annotation data from Gene Ontology and Reactome pathways, and data analysis tool enhancements. Nucleic Acids Res. 2017;45(D1):D183-D189. doi:10.1093/nar/gkw1138

Petri V, Jayaraman P, Tutaj M, Hayman GT, Smith JR, De Pons J, Laulederkind SJ, Lowry TF, Nigam R, Wang SJ et al. The pathway ontology - updates and applications. J Biomed Semantics. 2014;5(1):7. doi:10.1186/2041-1480-5-7

Jewison T, Su Y, Disfany FM, Liang Y, Knox C, Maciejewski A, Poelzer J, Huynh J, Zhou Y, Arndt D et al. SMPDB 2.0: big improvements to the Small Molecule Pathway Database. Nucleic Acids Res. 2014;42(Database issue):D478-D484. doi:10.1093/nar/gkt1067

Chen J, Bardes EE, Aronow BJ, Jegga AG.ToppGene Suite for gene list enrichment analysis and candidate gene prioritization. Nucleic Acids Res. 2009;37(Web Server issue):W305-W311. doi:10.1093/nar/gkp427

Bauer S. Gene-Category Analysis. Methods Mol Biol. 2017;1446:175-188. doi:10.1007/978-1-4939-3743-1_13 
medRxiv preprint doi: https://doi.org/10.1101/2020.12.21.20248688; this version posted December 24, 2020. The copyright holder for this preprint (which was not certified by peer review) is the author/funder, who has granted medRxiv a license to display the preprint in perpetuity. All rights reserved. No reuse allowed without permission.

Orchard S, Ammari M, Aranda B, Breuza L, Briganti L, Broackes-Carter F, Campbell NH, Chavali G, Chen C, del-Toro N et al. The MIntAct project-IntAct as a common curation platform for 11 molecular interaction databases. Nucleic Acids Res. 2014;42(Database issue):D358-D363. doi:10.1093/nar/gkt1115

Bateman A, Martin MJ, O'Donovan C, Magrane M, Apweiler R, Alpi E, Antunes $\mathrm{R}$, Arganiska J, Bely B, Bingley $\mathrm{M}$ et al. UniProt: a hub for protein information. Nucleic Acids Res. 2015;43(Database issue):D204-D212. doi:10.1093/nar/gku989

Brown KR, Jurisica I. Unequal evolutionary conservation of human protein interactions in interologous networks. Genome Biol. 2007;8(5):R95. doi:10.1186/gb-2007-8-5-r95

Breuer K, Foroushani AK, Laird MR, Chen C, Sribnaia A, Lo R, Winsor GL, Hancock RE, Brinkman FS, Lynn DJ. InnateDB: systems biology of innate immunity and beyond--recent updates and continuing curation. Nucleic Acids Res. 2013;41(Database issue):D1228-D233. doi:10.1093/nar/gks1147

Clerc O, Deniaud M, Vallet SD, Naba A, Rivet A, Perez S, Thierry-Mieg N, Ricard-Blum S. MatrixDB: integration of new data with a focus on glycosaminoglycan interactions. Nucleic Acids Res. 2019;47(D1):D376-D381. doi:10.1093/nar/gky1035

Orchard S, Kerrien S, Abbani S, Aranda B, Bhate J, Bidwell S, Bridge A, Briganti L, Brinkman FS, Cesareni $G$ et al. Protein interaction data curation: the International Molecular Exchange (IMEx) consortium. Nat Methods. 2012;9(4):345-350. doi:10.1038/nmeth.1931

Shannon P., Markiel A., Ozier O., Baliga N.S., Wang J.T., Ramage D. et al. 2003 Cytoscape: a software environment for integrated models of biomolecular interaction networks. Genome. Res. 3,2498-504. doi:10.1101/gr.1239303

Brown KR, Jurisica I. Unequal evolutionary conservation of human protein interactions in interologous networks. Genome Biol. 2007;8(5):R95. doi:10.1186/gb-2007-8-5-r95 
medRxiv preprint doi: https://doi.org/10.1101/2020.12.21.20248688; this version posted December 24, 2020. The copyright holder for this

Hormozdiari F, Berenbrink P, Przulj N, Sahinalp SC. Not all scale-free networks are born equal: the role of the seed graph in PPI network evolution. PLoS Comput Biol. 2007;3(7):e118. doi:10.1371/journal.pcbi.0030118

Bi D, Ning H, Liu S, Que X, Ding K. Gene expression patterns combined with network analysis identify hub genes associated with bladder cancer. Comput Biol Chem. 2015;56:71-83. doi:10.1016/j.compbiolchem.2015.04.001

Shi Z, Zhang B. Fast network centrality analysis using GPUs. BMC Bioinformatics. 2011;12:149. doi:10.1186/1471-2105-12-149

Stelzl U, Worm U, Lalowski M, Haenig C, Brembeck FH, Goehler H, Stroedicke M, Zenkner M, Schoenherr A, Koeppen S et al. A human protein-protein interaction network: a resource for annotating the proteome. Cell. 2005;122(6):957-968. doi:10.1016/j.cell.2005.08.029

Zaki N., Efimov D. and Berengueres J. 2013 Protein complex detection using interaction reliability assessment and weighted clustering coefficient. BMC. Bioinformatics. 14,163. doi:10.1186/1471-2105-14-163

Fan Y, Xia J miRNet-Functional Analysis and Visual Exploration of miRNATarget Interactions in a Network Context. Methods Mol Biol. 2018;1819:215-233. doi:10.1007/978-1-4939-8618-7_10

Vlachos IS, Paraskevopoulou MD, Karagkouni D, Georgakilas G, Vergoulis T, Kanellos I, Anastasopoulos IL, Maniou S, Karathanou K, Kalfakakou D et al DIANA-TarBase v7.0: indexing more than half a million experimentally supported miRNA:mRNA interactions. Nucleic Acids Res. 2015;43(Database issue):D153D159. doi:10.1093/nar/gku1215

Chou CH, Shrestha S, Yang CD, Chang NW, Lin YL, Liao KW, Huang WC, Sun TH, Tu SJ, Lee WH et al miRTarBase update 2018: a resource for experimentally validated microRNA-target interactions. Nucleic Acids Res. 2018;46(D1):D296D302. doi:10.1093/nar/gkx1067

Xiao F, Zuo Z, Cai G, Kang S, Gao X, Li T. miRecords: an integrated resource for microRNA-target interactions. Nucleic Acids Res. 2009;37(Database issue):D105D110. doi:10.1093/nar/gkn851 
medRxiv preprint doi: https://doi.org/10.1101/2020.12.21.20248688; this version posted December 24, 2020. The copyright holder for this preprint (which was not certified by peer review) is the author/funder, who has granted medRxiv a license to display the preprint in perpetuity. All rights reserved. No reuse allowed without permission.

Jiang Q, Wang Y, Hao Y, Juan L, Teng M, Zhang X, Li M, Wang G, Liu Y. miR2Disease: a manually curated database for microRNA deregulation in human disease. Nucleic Acids Res. 2009;37(Database issue):D98-104. doi:10.1093/nar/gkn714

Huang Z, Shi J, Gao Y, Cui C, Zhang S, Li J, Zhou Y, Cui Q. HMDD v3.0: a database for experimentally supported human microRNA-disease associations. Nucleic Acids Res. 2019;47(D1):D1013-D1017. doi:10.1093/nar/gky1010Z

Ruepp A, Kowarsch A, Schmidl D, Buggenthin F, Brauner B, Dunger I, Fobo G, Frishman G, Montrone C, Theis FJ. PhenomiR: a knowledgebase for microRNA expression in diseases and biological processes. Genome Biol. 2010;11(1):R6. doi:10.1186/gb-2010-11-1-r6

Liu X, Wang S, Meng F, Wang J, Zhang Y, Dai E, Yu X, Li X, Jiang W. SM2miR: a database of the experimentally validated small molecules' effects on microRNA expression. Bioinformatics. 2013;29(3):409-411. doi:10.1093/bioinformatics/bts698

Rukov JL, Wilentzik R, Jaffe I, Vinther J, Shomron N. Pharmaco-miR: linking microRNAs and drug effects. Brief Bioinform. 2014;15(4):648-659. doi:10.1093/bib/bbs082

Dai E, Yu X, Zhang Y, Meng F, Wang S, Liu X, Liu D, Wang J, Li X, Jiang W. EpimiR: a database of curated mutual regulation between miRNAs and epigenetic modifications. Database (Oxford). 2014;2014:bau023. doi:10.1093/database/bau023

Li JH, Liu S, Zhou H, Qu LH, Yang JH. starBase v2.0: decoding miRNA-ceRNA, miRNA-ncRNA and protein-RNA interaction networks from large-scale CLIP-Seq data. Nucleic Acids Res. 2014;42(Database issue):D92-D97. doi:10.1093/nar/gkt1248

Zhou G, Soufan O, Ewald J, Hancock REW, Basu N, Xia J. NetworkAnalyst 3.0: a visual analytics platform for comprehensive gene expression profiling and metaanalysis. Nucleic Acids Res. 2019. doi:10.1093/nar/gkz240 
medRxiv preprint doi: https://doi.org/10.1101/2020.12.21.20248688; this version posted December 24, 2020. The copyright holder for this preprint (which was not certified by peer review) is the author/funder, who has granted medRxiv a license to display the preprint in perpetuity. All rights reserved. No reuse allowed without permission.

Lachmann A, Xu H, Krishnan J, Berger SI, Mazloom AR, Ma'ayan A. (2010) ChEA: transcription factor regulation inferred from integrating genome-wide ChIP-X experiments. Bioinformatics. 2010;26(19):2438-2444. doi:10.1093/bioinformatics/btq466

Robin X, Turck N, Hainard A, Tiberti N, Lisacek F, Sanchez JC, Müller M. pROC: an open-source package for $\mathrm{R}$ and $\mathrm{S}+$ to analyze and compare ROC curves. BMC Bioinformatics. 2011;12:77. doi:10.1186/1471-2105-12-77

Gao L, Li C, Yang RY, Lian WW, Fang JS, Pang XC, Qin XM, Liu AL, Du GH. Ameliorative effects of baicalein in MPTP-induced mouse model of Parkinson's disease: A microarray study. Pharmacol Biochem Behav. 2015;133:155-163. doi:10.1016/j.pbb.2015.04.004

Zhao Y, Tan W, Sheng W, Li X. Identification of Biomarkers Associated With Alzheimer's Disease by Bioinformatics Analysis. Am J Alzheimers Dis Other Demen. 2016;31(2):163-168. doi:10.1177/1533317515588181

Ladogana A, Puopolo M, Croes EA, Budka H, Jarius C, Collins S, Klug GM, Sutcliffe T, Giulivi A, Alperovitch A et al. Mortality from Creutzfeldt-Jakob disease and related disorders in Europe, Australia, and Canada. Neurology. 2005;64(9):1586-1591. doi:10.1212/01.WNL.0000160117.56690.B2

Vallortigara J, Whitfield D, Quelch W, Alghamdi A, Howlett D, Hortobágyi T, Johnson M, Attems J, O'Brien JT, Thomas A et al. Decreased Levels of VAMP2 and Monomeric Alpha-Synuclein Correlate with Duration of Dementia. J Alzheimers Dis. 2016;50(1):101-110. doi:10.3233/JAD-150707

Zhou M, Yang WL, Ji Y, Qiang X, Wang P. Cold-inducible RNA-binding protein mediates neuroinflammation in cerebral ischemia. Biochim Biophys Acta. 2014;1840(7):2253-2261. doi:10.1016/j.bbagen.2014.02.027

Francelle L, Galvan L, Gaillard MC, Guillermier M, Houitte D, Bonvento G, Petit F, Jan C, Dufour N, Hantraye P et al. Loss of the thyroid hormone-binding protein Crym renders striatal neurons more vulnerable to mutant huntingtin in Huntington's disease. Hum Mol Genet. 2015;24(6):1563-1573. doi:10.1093/hmg/ddu571 
medRxiv preprint doi: https://doi.org/10.1101/2020.12.21.20248688; this version posted December 24, 2020. The copyright holder for this

Silva MG, Elliott K, Dahl HH, Fitzpatrick E, Wilcox S, Delatycki M, Williamson R, Efron D, Lynch M, Forrest S. Disruption of a novel member of a sodium/hydrogen exchanger family and DOCK3 is associated with an attention deficit hyperactivity disorder-like phenotype. J Med Genet. 2003;40(10):733-740. doi:10.1136/jmg.40.10.733

Tiwari SS, Mizuno K, Ghosh A, Aziz W, Troakes C, Daoud J, Golash V, Noble W, Hortobágyi T, Giese KP. Alzheimer-related decrease in CYFIP2 links amyloid production to tau hyperphosphorylation and memory loss. Brain. 2016;139(Pt 10):2751-2765. doi:10.1093/brain/aww205

Tiwari V, Patel AB. Pyruvate carboxylase and pentose phosphate fluxes are reduced in A $\beta P P-P S 1$ mouse model of Alzheimer's disease: a ${ }^{13} \mathrm{C}$ NMR study. $\mathrm{J}$ Alzheimers Dis. 2014;41(2):387-399. doi:10.3233/JAD-122449

Chong MS, Goh LK, Lim WS, Chan M, Tay L, Chen G, Feng L, Ng TP, Tan CH, Lee TS. Gene expression profiling of peripheral blood leukocytes shows consistent longitudinal downregulation of TOMM40 and upregulation of KIR2DL5A, PLOD1, and SLC2A8 among fast progressors in early Alzheimer's disease. J Alzheimers Dis. 2013;34(2):399-405. doi:10.3233/JAD-121621

Tsai CK, Huang LC, Tsai WC, Huang SM, Lee JT, Hueng DY. Overexpression of PLOD3 promotes tumor progression and poor prognosis in gliomas. Oncotarget. 2018;9(21):15705-15720. doi:10.18632/oncotarget.24594

Liu Y, Zhou Q, Tang M, Fu N, Shao W, Zhang S, Yin Y, Zeng R, Wang X, Hu G et al. Upregulation of alphaB-crystallin expression in the substantia nigra of patients with Parkinson's disease. Neurobiol Aging. 2015;36(4):1686-1691. doi:10.1016/j.neurobiolaging.2015.01.015

Durrenberger PF, Filiou MD, Moran LB, Michael GJ, Novoselov S, Cheetham ME, Clark P, Pearce RK, Graeber MB. DnaJB6 is present in the core of Lewy bodies and is highly up-regulated in parkinsonian astrocytes. J Neurosci Res. 2009;87(1):238-245. doi:10.1002/jnr.21819

Maeshima H, Ohnuma T, Sakai Y, Shibata N, Baba H, Ihara H, Higashi M, Ohkubo T, Nozawa E, Abe $\mathrm{S}$ et al. Increased plasma glutamate by antipsychotic medication and its relationship to glutaminase 1 and 2 genotypes in schizophrenia - 
medRxiv preprint doi: https://doi.org/10.1101/2020.12.21.20248688; this version posted December 24, 2020. The copyright holder for this

- Juntendo University Schizophrenia Projects (JUSP). Prog Neuropsychopharmacol Biol Psychiatry. 2007;31(7):1410-1418. doi:10.1016/j.pnpbp.2007.06.009

Tao R, Li C, Newburn EN, Ye T, Lipska BK, Herman MM, Weinberger DR, Kleinman JE, Hyde TM. Transcript-specific associations of SLC12A5 (KCC2) in human prefrontal cortex with development, schizophrenia, and affective disorders. J Neurosci. 2012;32(15):5216-5222. doi:10.1523/JNEUROSCI.4626-11.2012

Akbarian S, Huang HS. Molecular and cellular mechanisms of altered GAD1/GAD67 expression in schizophrenia and related disorders. Brain Res Rev. 2006;52(2):293-304. doi:10.1016/j.brainresrev.2006.04.001

Davis KN, Tao R, Li C, Gao Y, Gondré-Lewis MC, Lipska BK, Shin JH, Xie B, Ye T, Weinberger DR et al. GAD2 Alternative Transcripts in the Human Prefrontal Cortex, and in Schizophrenia and Affective Disorders. PLoS One. 2016;11(2):e0148558. doi:10.1371/journal.pone.0148558

Behan AT, Byrne C, Dunn MJ, Cagney G, Cotter DR. Proteomic analysis of membrane microdomain-associated proteins in the dorsolateral prefrontal cortex in schizophrenia and bipolar disorder reveals alterations in LAMP, STXBP1 and BASP1 protein expression. Mol Psychiatry. 2009;14(6):601-613. doi:10.1038/mp.2008.7

Saito A, Fujikura-Ouchi Y, Kuramasu A, Shimoda K, Akiyama K, Matsuoka H, Ito C. Association study of putative promoter polymorphisms in the neuroplastin gene and schizophrenia. Neurosci Lett. 2007;411(3):168-173. doi:10.1016/j.neulet.2006.08.042

Liu CM, Fann CS, Chen CY, Liu YL, Oyang YJ, Yang WC, Chang CC, Wen CC, Chen WJ, Hwang TJ et al. ANXA7, PPP3CB, DNAJC9, and ZMYND17 genes at chromosome 10q22 associated with the subgroup of schizophrenia with deficits in attention and executive function. Biol Psychiatry. 2011;70(1):51-58. doi:10.1016/j.biopsych.2011.02.033

Huang Y, Zhao L, Jia B, Wu L, Li Y, Curthoys N, Zheng JC. Glutaminase dysregulation in HIV-1-infected human microglia mediates neurotoxicity: relevant 
medRxiv preprint doi: https://doi.org/10.1101/2020.12.21.20248688; this version posted December 24, 2020. The copyright holder for this preprint (which was not certified by peer review) is the author/funder, who has granted medRxiv a license to display the preprint in perpetuity. All rights reserved. No reuse allowed without permission.

to HIV-1-associated neurocognitive disorders. J Neurosci. 2011;31(42):15195-204. doi:10.1523/JNEUROSCI.2051-11.2011

Shamseldin HE, Masuho I, Alenizi A, Alyamani S, Patil DN, Ibrahim N, Martemyanov KA, Alkuraya FS. GNB5 mutation causes a novel neuropsychiatric disorder featuring attention deficit hyperactivity disorder, severely impaired language development and normal cognition. Genome Biol. 2016;17(1):195. doi:10.1186/s13059-016-1061-6

Antonell A, Lladó A, Sánchez-Valle R, Sanfeliu C, Casserras T, Rami L, MuñozGarcía C, Dangla-Valls A, Balasa M, Boya P et al. Altered Blood Gene Expression of Tumor-Related Genes (PRKCB, BECN1, and CDKN2A) in Alzheimer's Disease. Mol Neurobiol. 2016;53(9):5902-5911. doi:10.1007/s12035-015-9483-9

Brinkmalm A, Brinkmalm G, Honer WG, Frölich L, Hausner L, Minthon L, Hansson O, Wallin A, Zetterberg H, Blennow $\mathrm{K}$ et al. SNAP-25 is a promising novel cerebrospinal fluid biomarker for synapse degeneration in Alzheimer's disease. Mol Neurodegener. 2014;9:53. doi:10.1186/1750-1326-9-53

Ibarreta D, Tao J, Parrilla R, Ayuso MS. Mutation analysis of chromosome 19 calmodulin (CALM3) gene in Alzheimer's disease patients. Neurosci Lett. 1997;229(3):157-160. doi:10.1016/s0304-3940(97)00453-9

Liu QY, Lei JX, Sikorska M, Liu R. A novel brain-enriched E3 ubiquitin ligase RNF182 is up regulated in the brains of Alzheimer's patients and targets ATP6V0C for degradation. Mol Neurodegener. 2008;3:4. doi:10.1186/1750-1326-3-4

Gahete MD, Rubio A, Durán-Prado M, Avila J, Luque RM, Castaño JP. Expression of Somatostatin, cortistatin, and their receptors, as well as dopamine receptors, but not of neprilysin, are reduced in the temporal lobe of Alzheimer's disease patients. J Alzheimers Dis. 2010;20(2):465-475. doi:10.3233/JAD-20101385

Seki T, Takahashi H, Adachi N, Abe N, Shimahara T, Saito N, Sakai N. Aggregate formation of mutant protein kinase $\mathrm{C}$ gamma found in spinocerebellar ataxia type 14 impairs ubiquitin-proteasome system and induces endoplasmic reticulum stress. Eur J Neurosci. 2007;26(11):3126-3140. doi:10.1111/j.1460-9568.2007.05933.x 
medRxiv preprint doi: https://doi.org/10.1101/2020.12.21.20248688; this version posted December 24, 2020. The copyright holder for this preprint (which was not certified by peer review) is the author/funder, who has granted medRxiv a license to display the preprint in perpetuity. All rights reserved. No reuse allowed without permission.

Leemput J, Chandran J, Knight MA, Holtzclaw LA, Scholz S, Cookson MR, Houlden H, Gwinn-Hardy K, Fung HC, Lin X et al. Deletion at ITPR1 underlies ataxia in mice and spinocerebellar ataxia 15 in humans. PLoS Genet. 2007;3(6):e108. doi:10.1371/journal.pgen.0030108

Bourassa CV, Meijer IA, Merner ND, Grewal KK, Stefanelli MG, Hodgkinson K, Ives EJ, Pryse-Phillips W, Jog M, Boycott $\mathrm{K}$ et al. VAMP1 mutation causes dominant hereditary spastic ataxia in Newfoundland families. Am J Hum Genet. 2012;91(3):548-552. doi:10.1016/j.ajhg.2012.07.018

Cossette P, Liu L, Brisebois K, Dong H, Lortie A, Vanasse M, Saint-Hilaire JM, Carmant L, Verner A, Lu WY et al. Mutation of GABRA1 in an autosomal dominant form of juvenile myoclonic epilepsy. Nat Genet. 2002;31(2):184-189. doi:10.1038/ng885

Lü JJ, Zhang YH, Pan H, Chen YC, Liu XY, Jiang YW, Bao XH, Shen Y, Wu HS, $\mathrm{Xu} \mathrm{KM}$ et al. Case-control study and transmission/disequilibrium tests of the genes encoding GABRA5 and GABRB3 in a Chinese population affected by childhood absence epilepsy. Chin Med J (Engl). 2004;117(10):1497-501.

Kang JQ, Macdonald RL. Molecular Pathogenic Basis for GABRG2 Mutations Associated With a Spectrum of Epilepsy Syndromes, From Generalized Absence Epilepsy to Dravet Syndrome. JAMA Neurol. 2016;73(8):1009-1016. doi:10.1001/jamaneurol.2016.0449

Kulkarni N, Tang S, Bhardwaj R, Bernes S, Grebe TA. Progressive Movement Disorder in Brothers Carrying a GNAO1 Mutation Responsive to Deep Brain Stimulation. J Child Neurol. 2016;31(2):211-214. doi:10.1177/0883073815587945

Nakamura K, Iwata Y, Anitha A, Miyachi T, Toyota T, Yamada S, Tsujii M, Tsuchiya KJ, Iwayama Y, Yamada K et al. Replication study of Japanese cohorts supports the role of STX1A in autism susceptibility. Prog Neuropsychopharmacol Biol Psychiatry. 2011;35(2):454-458. doi:10.1016/j.pnpbp.2010.11.033

Mizuno M, Matsumoto A, Hamada N, Ito H, Miyauchi A, Jimbo EF, Momoi MY, Tabata H, Yamagata T, Nagata K. Role of an adaptor protein Lin-7B in brain development: possible involvement in autism spectrum disorders. J Neurochem. 2015;132(1):61-69. doi:10.1111/jnc.12943 
medRxiv preprint doi: https://doi.org/10.1101/2020.12.21.20248688; this version posted December 24, 2020. The copyright holder for this preprint (which was not certified by peer review) is the author/funder, who has granted medRxiv a license to display the preprint in perpetuity. All rights reserved. No reuse allowed without permission.

Breitenkamp AF, Matthes J, Nass RD, Sinzig J, Lehmkuhl G, Nürnberg P, Herzig $\mathrm{S}$. Rare mutations of CACNB2 found in autism spectrum disease-affected families alter calcium channel function. PLoS One. 2014;9(4):e95579. doi:10.1371/journal.pone.0095579

Thompson AGB, Luk C, Heslegrave AJ, Zetterberg H, Mead SH, Collinge J, Jackson GS. Neurofilament light chain and tau concentrations are markedly increased in the serum of patients with sporadic Creutzfeldt-Jakob disease, and tau correlates with rate of disease progression. J Neurol Neurosurg Psychiatry. 2018;89(9):955-961. doi:10.1136/jnnp-2017-317793

Yun J, Jeong BH, Kim HJ, Park YJ, Lee YJ, Choi EK, Carp RI, Kim YS. A polymorphism in the YWHAH gene encoding 14-3-3 eta that is not associated with sporadic Creutzfeldt-Jakob disease (CJD). Mol Biol Rep. 2012;39(4):3619-3625. doi:10.1007/s11033-011-1136-0

Gonda X, Eszlari N, Anderson IM, Deakin JF, Bagdy G, Juhasz G. Association of ATP6V1B2 rs1106634 with lifetime risk of depression and hippocampal neurocognitive deficits: possible novel mechanisms in the etiopathology of depression. Transl Psychiatry. 2016;6(11):e945. doi:10.1038/tp.2016.221

Lee J, Hwang YJ, Shin JY, Lee WC, Wie J, Kim KY, Lee MY, Hwang D, Ratan RR, Pae AN et al. Epigenetic regulation of cholinergic receptor M1 (CHRM1) by histone $\mathrm{H} 3 \mathrm{~K} 9 \mathrm{me} 3$ impairs $\mathrm{Ca}(2+)$ signaling in Huntington's disease. Acta Neuropathol. 2013;125(5):727-739. doi:10.1007/s00401-013-1103-z

Wang J, Si YM, Liu ZL, Yu L. Cholecystokinin, cholecystokinin-A receptor and cholecystokinin-B receptor gene polymorphisms in Parkinson's disease. Pharmacogenetics. 2003;13(6):365-369. doi:10.1097/01.fpc.0000054095.48725.fe

Takehashi M, Tanaka S, Masliah E, Ueda K. Association of monoamine oxidase A gene polymorphism with Alzheimer's disease and Lewy body variant. Neurosci Lett. 2002;327(2):79-82. doi:10.1016/s0304-3940(02)00258-6

Peress NS, Perillo E. Differential expression of TGF-beta 1, 2 and 3 isotypes in Alzheimer's disease: a comparative immunohistochemical study with cerebral infarction, aged human and mouse control brains. J Neuropathol Exp Neurol. 1995;54(6):802-811. doi:10.1097/00005072-199511000-00007 
medRxiv preprint doi: https://doi.org/10.1101/2020.12.21.20248688; this version posted December 24, 2020. The copyright holder for this preprint (which was not certified by peer review) is the author/funder, who has granted medRxiv a license to display the preprint in perpetuity. All rights reserved. No reuse allowed without permission.

Ko CY, Chu YY, Narumiya S, Chi JY, Furuyashiki T, Aoki T, Wang SM, Chang WC, Wang JM. CCAAT/enhancer-binding protein delta/miR135a/thrombospondin 1 axis mediates PGE2-induced angiogenesis in Alzheimer's disease. Neurobiol Aging. 2015;36(3):1356-1368. doi:10.1016/j.neurobiolaging.2014.11.020

Mitchelmore C, Büchmann-Møller S, Rask L, West MJ, Troncoso JC, Jensen NA. NDRG2: a novel Alzheimer's disease associated protein. Neurobiol Dis. 2004;16(1):48-58. doi:10.1016/j.nbd.2004.01.003

Jamieson SE, White JK, Howson JM, Pask R, Smith AN, Brayne C, Evans JG, Xuereb J, Cairns NJ, Rubinsztein DC et al. Candidate gene association study of solute carrier family 11a members 1 (SLC11A1) and 2 (SLC11A2) genes in Alzheimer's disease. Neurosci Lett. 2005;374(2):124-128. doi:10.1016/j.neulet.2004.10.038

Zhang J, Zhang R, Zhan Z, Li X, Zhou F, Xing A, Jiang C, Chen Y, An L. Beneficial Effects of Sulforaphane Treatment in Alzheimer's Disease May Be Mediated through Reduced HDAC1/3 and Increased P75NTR Expression. Front Aging Neurosci. 2017;9:121. doi:10.3389/fnagi.2017.00121

Ma J, Jiang T, Tan L, Yu JT. TYROBP in Alzheimer's disease. Mol Neurobiol. 2015;51(2):820-826. doi:10.1007/s12035-014-8811-9

Manev H, Manev R. 5-Lipoxygenase (ALOX5) and FLAP (ALOX5AP) gene polymorphisms as factors in vascular pathology and Alzheimer's disease. Med Hypotheses. 2006;66(3):501-503. doi:10.1016/j.mehy.2005.09.031

Cimino PJ, Sokal I, Leverenz J, Fukui Y, Montine TJ. DOCK2 is a microglial specific regulator of central nervous system innate immunity found in normal and Alzheimer's disease brain. Am J Pathol. 2009;175(4):1622-1630. doi:10.2353/ajpath.2009.090443

Kong Y, Wu JB, Wang X, Zhao JF, Song H, Yuan LD. Polymorphism of the OLR1 3'UTR potential microRNA binding site and risk of Alzheimer's disease: a meta-analysis. Genet Mol Res. 2014;13(4):10162-10172. doi:10.4238/2014

Fassbender K, Walter S, Kühl S, Landmann R, Ishii K, Bertsch T, Stalder AK, Muehlhauser F, Liu Y, Ulmer AJ et al. The LPS receptor (CD14) links innate 
medRxiv preprint doi: https://doi.org/10.1101/2020.12.21.20248688; this version posted December 24, 2020. The copyright holder for this preprint (which was not certified by peer review) is the author/funder, who has granted medRxiv a license to display the preprint in perpetuity. All rights reserved. No reuse allowed without permission.

immunity with Alzheimer's disease. FASEB J. 2004;18(1):203-205. doi:10.1096/fj.03-0364fje

Akiyama H, Tooyama I, Kawamata T, Ikeda K, McGeer PL. Morphological diversities of CD44 positive astrocytes in the cerebral cortex of normal subjects and patients with Alzheimer's disease. Brain Res. 1993;632(1-2):249-259. doi:10.1016/0006-8993(93)91160-t

Matsumura A, Suzuki S, Iwahara N, Hisahara S, Kawamata J, Suzuki H, Yamauchi A, Takata K, Kitamura Y, Shimohama S. Temporal changes of CD68 and $\alpha 7$ nicotinic acetylcholine receptor expression in microglia in Alzheimer's disease-like mouse models. J Alzheimers Dis. 2015;44(2):409-423. doi:10.3233/JAD-141572

Salta E, Sierksma A, Vanden Eynden E, De Strooper B. miR-132 loss de-represses ITPKB and aggravates amyloid and TAU pathology in Alzheimer's brain. EMBO Mol Med. 2016;8(9):1005-1018. doi:10.15252/emmm.201606520

Kitamura Y, Tsuchiya D, Takata K, Shibagaki K, Taniguchi T, Smith MA, Perry G, Miki H, Takenawa T, Shimohama S. Possible involvement of Wiskott-Aldrich syndrome protein family in aberrant neuronal sprouting in Alzheimer's disease. Neurosci Lett. 2003;346(3):149-152. doi:10.1016/s0304-3940(03)00506-8

Busse S, Steiner J, Alter J, Dobrowolny H, Mawrin C, Bogerts B, Hartig R, Busse M. Expression of HLA-DR, CD80, and CD86 in Healthy Aging and Alzheimer's Disease. J Alzheimers Dis. 2015;47(1):177-184. doi:10.3233/JAD-150217

Bryan KJ, Zhu X, Harris PL, Perry G, Castellani RJ, Smith MA, Casadesus G. Expression of CD74 is increased in neurofibrillary tangles in Alzheimer's disease. Mol Neurodegener. 2008;3:13. doi:10.1186/1750-1326-3-13

Alves-Leon SV, Veluttini-Pimentel ML, Gouveia ME, Malfetano FR, Gaspareto EL, Alvarenga MP, Frugulhetti I, Quirico-Santos T. Acute disseminated encephalomyelitis: clinical features, HLA DRB1*1501, HLA DRB1*1503, HLA DQA1*0102, HLA DQB1*0602, and HLA DPA1*0301 allelic association study. Arq Neuropsiquiatr. 2009;67(3A):643-651. 
medRxiv preprint doi: https://doi.org/10.1101/2020.12.21.20248688; this version posted December 24, 2020. The copyright holder for this preprint (which was not certified by peer review) is the author/funder, who has granted medRxiv a license to display the preprint in perpetuity. All rights reserved. No reuse allowed without permission.

Haegert DG, Muntoni F, Murru MR, Costa G, Francis GS, Marrosu MG. HLADQA1 and -DQB1 associations with multiple sclerosis in Sardinia and French Canada: evidence for immunogenetically distinct patient groups. Neurology. 1993;43(3 Pt 1):548-552. doi:10.1212/wnl.43.3_part_1.548

De Jager PL, Jia X, Wang J, de Bakker PI, Ottoboni L, Aggarwal NT, Piccio L, Raychaudhuri S, Tran D, Aubin C et al. Meta-analysis of genome scans and replication identify CD6, IRF8 and TNFRSF1A as new multiple sclerosis susceptibility loci. Nat Genet. 2009;41(7):776-782. doi:10.1038/ng.401

Strickland MR, Koller EJ, Deng DZ, Ceballos-Diaz C, Golde TE, Chakrabarty P. Ifngr1 and Stat1 mediated canonical Ifn- $\gamma$ signaling drives nigrostriatal degeneration. Neurobiol Dis. 2018;110:133-141. doi:10.1016/j.nbd.2017.11.007

Yun HM, Choi DY, Oh KW, Hong JT. PRDX6 Exacerbates Dopaminergic Neurodegeneration in a MPTP Mouse Model of Parkinson's Disease. Mol Neurobiol. 2015;52(1):422-431. doi:10.1007/s12035-014-8885-4

Pang S, Chen D, Zhang A, Qin X, Yan B. Genetic analysis of the LAMP-2 gene promoter in patients with sporadic Parkinson's disease. Neurosci Lett. 2012;526(1):63-67. doi:10.1016/j.neulet.2012.07.044

Han L, Wang L, Tang S, Yuan L, Wu S, Du X, Xiang Y, Qu X, Liu H, Luo H et al. ITGB4 deficiency in bronchial epithelial cells directs airway inflammation and bipolar disorder-related behavior. J Neuroinflammation. 2018;15(1):246. doi:10.1186/s12974-018-1283-5

Vanni S, Moda F, Zattoni M, Bistaffa E, De Cecco E, Rossi M, Giaccone G, Tagliavini F, Haïk S, Deslys JP et al. Differential overexpression of SERPINA3 in human prion diseases. Sci Rep. 2017;7(1):15637. doi:10.1038/s41598-017-157788

Fan HW, Ni Q, Fan YN, Ma ZX, Li YB. C-type lectin domain family 5, member A (CLEC5A, MDL-1) promotes brain glioblastoma tumorigenesis by regulating PI3K/Akt signalling. Cell Prolif. 2019;52(3):e12584. doi:10.1111/cpr.12584

Pae CU, Kim TS, Kwon OJ, Artioli P, Serretti A, Lee CU, Lee SJ, Lee C, Paik IH, Kim JJ. Polymorphisms of heat shock protein 70 gene (HSPA1A, HSPA1B and 
medRxiv preprint doi: https://doi.org/10.1101/2020.12.21.20248688; this version posted December 24, 2020. The copyright holder for this preprint (which was not certified by peer review) is the author/funder, who has granted medRxiv a license to display the preprint in perpetuity. All rights reserved. No reuse allowed without permission.

HSPA1L) and schizophrenia. Neurosci Res. 2005;53(1):8-13. doi:10.1016/j.neures.2005.05.004

Liou HL, Dixit SS, Xu S, Tint GS, Stock AM, Lobel P. NPC2, the protein deficient in Niemann-Pick C2 disease, consists of multiple glycoforms that bind a variety of sterols. J Biol Chem. 2006;281(48):36710-36723. doi:10.1074/jbc.M608743200

Sinkevicius KW, Morrison TR, Kulkarni P, Caffrey Cagliostro MK, Iriah S, Malmberg S, Sabrick J, Honeycutt JA, Askew KL et al. RNaseT2 knockout rats exhibit hippocampal neuropathology and deficits in memory. Dis Model Mech. 2018;11(6). doi:10.1242/dmm.032631

Carson R, McKnight AJ, Todd S, Liu WW, Heggarty S, Craig D, McGuinness B, Irvine GB, Passmore AP, Johnston JA. Variation in RTN3 and PPIL2 genes does not influence platelet membrane beta-secretase activity or susceptibility to alzheimer's disease in the northern Irish population. Neuromolecular Med. 2009;11(4):337-44. doi:10.1007/s12017-009-8080-3

Llano DA, Devanarayan P, Devanarayan V. VGF in Cerebrospinal Fluid Combined With Conventional Biomarkers Enhances Prediction of Conversion From MCI to AD. Alzheimer Dis Assoc Disord. 2019. doi:10.1097/WAD.0000000000000328

Shibasaki Y, Baillie DA, St Clair D, Brookes AJ. High-resolution mapping of SNCA encoding alpha-synuclein, the non-A beta component of Alzheimer's disease amyloid precursor, to human chromosome $4 \mathrm{q} 21.3-->\mathrm{q} 22$ by fluorescence in situ hybridization. Cytogenet Cell Genet. 1995;71(1):54-55. doi:10.1159/000134061

Liu SJ, Yang C, Zhang Y, Su RY, Chen JL, Jiao MM, Chen HF, Zheng N, Luo S, Chen YB et al. Neuroprotective effect of $\beta$-asarone against Alzheimer's disease: regulation of synaptic plasticity by increased expression of SYP and GluR1. Drug Des Devel Ther. 2016;10:1461-1469. doi:10.2147/DDDT.S93559

Ma QL, Teng E, Zuo X, Jones M, Teter B, Zhao EY, Zhu C, Bilousova T, Gylys $\mathrm{KH}$, Apostolova LG et al. Neuronal pentraxin 1: A synaptic-derived plasma biomarker in Alzheimer's disease. Neurobiol Dis. 2018;114:120-128. doi:10.1016/j.nbd.2018.02.014 
medRxiv preprint doi: https://doi.org/10.1101/2020.12.21.20248688; this version posted December 24, 2020. The copyright holder for this preprint (which was not certified by peer review) is the author/funder, who has granted medRxiv a license to display the preprint in perpetuity. All rights reserved. No reuse allowed without permission.

Tarawneh R, D'Angelo G, Crimmins D, Herries E, Griest T, Fagan AM, Zipfel GJ, Ladenson JH, Morris JC, Holtzman DM. Diagnostic and Prognostic Utility of the Synaptic Marker Neurogranin in Alzheimer Disease. JAMA Neurol. 2016;73(5):561-571. doi:10.1001/jamaneurol.2016.0086

Bakalash S, Pham M, Koronyo Y, Salumbides BC, Kramerov A, Seidenberg H, Berel D, Black KL, Koronyo-Hamaoui M. Egr1 expression is induced following glatiramer acetate immunotherapy in rodent models of glaucoma and Alzheimer's disease. Invest Ophthalmol Vis Sci. 2011;52(12):9033-9046. doi:10.1167/iovs.117498

Liu SL, Wang C, Jiang T, Tan L, Xing A, Yu JT. The Role of Cdk5 in Alzheimer's Disease. Mol Neurobiol. 2016;53(7):4328-4342. doi:10.1007/s12035-015-9369-x

Hasegawa M, Arai T, Ihara Y. Immunochemical evidence that fragments of phosphorylated MAP5 (MAP1B) are bound to neurofibrillary tangles in Alzheimer's disease. Neuron. 1990;4(6):909-918.

Zhang M, Cai F, Zhang S, Zhang S, Song W. Overexpression of ubiquitin carboxyl-terminal hydrolase L1 (UCHL1) delays Alzheimer's progression in vivo. Sci Rep. 2014;4:7298. doi:10.1038/srep07298

Wen L, Tang FL, Hong Y, Luo SW, Wang CL, He W, Shen C, Jung JU, Xiong F, Lee DH et al. VPS35 haploinsufficiency increases Alzheimer's disease neuropathology. J Cell Biol. 2011;195(5):765-779. doi:10.1083/jcb.201105109

Abel D, Michel O, Veerhuis R, Jacobs M, van Dijk M, Oudejans CB. Direct downregulation of CNTNAP2 by STOX1A is associated with Alzheimer's disease. J Alzheimers Dis. 2012;31(4):793-800. doi:10.3233/JAD-2012-120472

Traina G, Federighi G, Brunelli M. Up-regulation of kinesin light-chain 1 gene expression by acetyl-L-carnitine: therapeutic possibility in Alzheimer's disease. Neurochem Int. 2008;53(6-8):244-247. doi:10.1016/j.neuint.2008.08.001

Zhang L, Fang Y, Cheng X, Lian YJ, Xu HL. Silencing of Long Noncoding RNA SOX21-AS1 Relieves Neuronal Oxidative Stress Injury in Mice with Alzheimer's Disease by Upregulating FZD3/5 via the Wnt Signaling Pathway. Mol Neurobiol. 2019;56(5):3522-3537. doi:10.1007/s12035-018-1299-y 
medRxiv preprint doi: https://doi.org/10.1101/2020.12.21.20248688; this version posted December 24, 2020. The copyright holder for this preprint (which was not certified by peer review) is the author/funder, who has granted medRxiv a license to display the preprint in perpetuity. All rights reserved. No reuse allowed without permission.

Perry RH, Dockray GJ, Dimaline R, Perry EK, Blessed G, Tomlinson BE. Neuropeptides in Alzheimer's disease, depression and schizophrenia. A post mortem analysis of vasoactive intestinal peptide and cholecystokinin in cerebral cortex. J Neurol Sci. 1981;51(3):465-472. doi:10.1016/0022-510x(81)90123-4

Matsui C, Inoue E, Kakita A, Arita K, Deguchi-Tawarada M, Togawa A, Yamada A, Takai Y, Takahashi H. Involvement of the $\gamma$-secretase-mediated EphA4 signaling pathway in synaptic pathogenesis of Alzheimer's disease. Brain Pathol. 2012;22(6):776-787. doi:10.1111/j.1750-3639.2012.00587.x

Reumiller CM, Schmidt GJ, Dhrami I, Umlauf E, Rappold E, Zellner M. Genderrelated increase of tropomyosin-1 abundance in platelets of Alzheimer's disease and mild cognitive impairment patients. J Proteomics. 2018;178:73-81. doi:10.1016/j.jprot.2017.12.018

Watamura N, Toba J, Yoshii A, Nikkuni M, Ohshima T. Colocalization of phosphorylated forms of WAVE1, CRMP2, and tau in Alzheimer's disease model mice: Involvement of Cdk5 phosphorylation and the effect of ATRA treatment. J Neurosci Res. 2016;94(1):15-26. doi:10.1002/jnr.23674

Juneja M, Azmi A, Baets J, Roos A, Jennings MJ, Saveri P, Pisciotta C, BernardMarissal N, Schneider BL, Verfaillie $\mathrm{C}$ et al PFN2 and GAMT as common molecular determinants of axonal Charcot-Marie-Tooth disease. J Neurol Neurosurg Psychiatry. 2018;89(8):870-878. doi:10.1136/jnnp-2017-317562

Koga $\mathrm{M}$, Ishiguro $\mathrm{H}$, Horiuchi $\mathrm{Y}$, Inada $\mathrm{T}$, Ujike $\mathrm{H}$, Itokawa $\mathrm{M}$, Otowa $\mathrm{T}$, Watanabe Y, Someya T, Arinami T. Replication study of association between ADCYAP1 gene polymorphisms and schizophrenia. Psychiatr Genet. 2010;20(3):123-125. doi:10.1097/YPG.0b013e32833a1f52

Duong L, Klitten LL, Møller RS, Ingason A, Jakobsen KD, Skjødt C, Didriksen M, Hjalgrim H, Werge T, Tommerup N. Mutations in NRXN1 in a family multiply affected with brain disorders: NRXN1 mutations and brain disorders. Am J Med Genet B Neuropsychiatr Genet. 2012;159B(3):354-358. doi:10.1002/ajmg.b.32036

Francks C, Maegawa S, Laurén J, Abrahams BS, Velayos-Baeza A, Medland SE, Colella S, Groszer M, McAuley EZ, Caffrey TM et al. LRRTM1 on chromosome 2 p12 is a maternally suppressed gene that is associated paternally with handedness 
medRxiv preprint doi: https://doi.org/10.1101/2020.12.21.20248688; this version posted December 24, 2020. The copyright holder for this preprint (which was not certified by peer review) is the author/funder, who has granted medRxiv a license to display the preprint in perpetuity. All rights reserved. No reuse allowed without permission.

and schizophrenia. Mol Psychiatry. 2007;12(12):1129-1139. doi:10.1038/sj.mp.4002053

Håvik B, Degenhardt FA, Johansson S, Fernandes CP, Hinney A, Scherag A, Lybæk H, Djurovic S, Christoforou A, Ersland KM et al. DCLK1 variants are associated across schizophrenia and attention deficit/hyperactivity disorder. PLoS One. 2012;7(4):e35424. doi:10.1371/journal.pone.0035424

Scheffer IE, Harkin LA, Grinton BE, Dibbens LM, Turner SJ, Zielinski MA, Xu R, Jackson G, Adams J, Connellan $M$ et al. Temporal lobe epilepsy and GEFS+ phenotypes associated with SCN1B mutations. Brain. 2007;130(Pt 1):100-109. doi:10.1093/brain/awl272

Guella I, Huh L, McKenzie MB, Toyota EB, Bebin EM, Thompson ML, Cooper GM, Evans DM, Buerki SE, Adam S et al De novo FGF12 mutation in 2 patients with neonatal-onset epilepsy. Neurol Genet. 2016;2(6):e120. doi:10.1212/NXG.0000000000000120

Conroy J, Allen NM, Gorman KM, Shahwan A, Ennis S, Lynch SA, King MD. NAPB - a novel SNARE-associated protein for early-onset epileptic encephalopathy. Clin Genet. 2016;89(2):E1-E3. doi:10.1111/cge.12648

Dhindsa RS, Bradrick SS, Yao X, Heinzen EL, Petrovski S, Krueger BJ, Johnson MR, Frankel WN, Petrou S, Boumil RM et al. Epileptic encephalopathy-causing mutations in DNM1 impair synaptic vesicle endocytosis. Neurol Genet. 2015;1(1):e4. doi:10.1212/01.NXG.0000464295.65736.da

Assoum M, Philippe C2, Isidor B3, Perrin L4, Makrythanasis P5, Sondheimer N6, Paris C7, Douglas J8, Lesca G9, Antonarakis S et al. Autosomal-Recessive Mutations in AP3B2, Adaptor-Related Protein Complex 3 Beta 2 Subunit, Cause an Early-Onset Epileptic Encephalopathy with Optic Atrophy. Am J Hum Genet. 2016;99(6):1368-1376. doi:10.1016/j.ajhg.2016.10.009

Brighina L, Okubadejo NU, Schneider NK, Lesnick TG, de Andrade M, Cunningham JM, Farrer MJ, Lincoln SJ, Rocca WA, Maraganore DM. Betasynuclein gene variants and Parkinson's disease: a preliminary case-control study. Neurosci Lett. 2007;420(3):229-234. doi:10.1016/j.neulet.2007.05.021 
medRxiv preprint doi: https://doi.org/10.1101/2020.12.21.20248688; this version posted December 24, 2020. The copyright holder for this preprint (which was not certified by peer review) is the author/funder, who has granted medRxiv a license to display the preprint in perpetuity. All rights reserved. No reuse allowed without permission.

Pankratz N, Beecham GW, DeStefano AL, Dawson TM, Doheny KF, Factor SA, Hamza TH, Hung AY, Hyman BT, Ivinson AJ et al. Meta-analysis of Parkinson's disease: identification of a novel locus, RIT2. Ann Neurol. 2012;71(3):370-384. doi:10.1002/ana.22687

Lorenzo-Betancor O, Wszolek ZK, Ross OA. Rare variants in MC1R/TUBB3 exon 1 are not associated with Parkinson's disease. Ann Neurol. 2016;79(2):331. doi:10.1002/ana.24581

Lanoue AC, Blatt GJ, Soghomonian JJ. Decreased parvalbumin mRNA expression in dorsolateral prefrontal cortex in Parkinson's disease. Brain Res. 2013;1531:3747. doi:10.1016/j.brainres.2013.07.025

Noureddine MA, Qin XJ, Oliveira SA, Skelly TJ, van der Walt J, Hauser MA, Pericak-Vance MA, Vance JM, Li YJ. Association between the neuron-specific RNA-binding protein ELAVL4 and Parkinson disease. Hum Genet. 2005;117(1):27-33. doi:10.1007/s00439-005-1259-2

Okamoto N, Hatsukawa Y, Shimojima K, Yamamoto T. Submicroscopic deletion in 7q31 encompassing CADPS2 and TSPAN12 in a child with autism spectrum disorder and PHPV. Am J Med Genet A. 2011;155A(7):1568-1573. doi:10.1002/ajmg.a.34028

Carayol J, Sacco R, Tores F, Rousseau F, Lewin P, Hager J, Persico AM. Converging evidence for an association of ATP2B2 allelic variants with autism in $\begin{array}{llll}\text { male subjects. } & \text { Biol } & \text { Psychiatry. }\end{array}$ doi:10.1016/j.biopsych.2011.05.020

Wang Y, Fang Y, Zhang F, Xu M, Zhang J, Yan J, Ju W, Brown WT, Zhong N. Hypermethylation of the enolase gene (ENO2) in autism. Eur J Pediatr. 2014;173(9):1233-1244. doi:10.1007/s00431-014-2311-9

Cunningham S, O'Doherty C, Patterson C, McDonnell G, Hawkins S, Marrosu MG, Vandenbroeck K. The neuropeptide genes TAC1, TAC3, TAC4, VIP and PACAP(ADCYAP1), and susceptibility to multiple sclerosis. J Neuroimmunol. 2007;183(1-2):208-213. doi:10.1016/j.jneuroim.2006.11.002 
medRxiv preprint doi: https://doi.org/10.1101/2020.12.21.20248688; this version posted December 24, 2020. The copyright holder for this preprint (which was not certified by peer review) is the author/funder, who has granted medRxiv a license to display the preprint in perpetuity. All rights reserved. No reuse allowed without permission.

Diez M, Koistinaho J, Dearmond SJ, Groth D, Prusiner SB, Hökfelt T. Marked decrease of neuropeptide Y Y2 receptor binding sites in the hippocampus in murine prion disease. Proc Natl Acad Sci U S A. 1997;94(24):13267-13272. doi:10.1073/pnas.94.24.13267

Guo Y, Gong HS, Zhang J, Xie WL, Tian C, Chen C, Shi Q, Wang SB, Xu Y, Zhang BY et al. Remarkable reduction of MAP2 in the brains of scrapie-infected rodents and human prion disease possibly correlated with the increase of calpain. PLoS One. 2012;7(1):e30163. doi:10.1371/journal.pone.0030163

Gavín R, Ferrer I, del Río JA. Involvement of Dab1 in APP processing and betaamyloid deposition in sporadic Creutzfeldt-Jakob patients. Neurobiol Dis. 2010;37(2):324-329. doi:10.1016/j.nbd.2009.10.010

Jeong BH, Kim HJ, Lee KH, Carp RI, Kim YS. RARB and STMN2 polymorphisms are not associated with sporadic Creutzfeldt-Jakob disease (CJD) in the Korean population. Mol Biol Rep. 2014;41(4):2389-2395. doi:10.1007/s11033-014-3093-x

Peoc'h K, Manivet P, Beaudry P, Attane F, Besson G, Hannequin D, DelasnerieLauprêtre N, Laplanche JL. Identification of three novel mutations (E196K, V203I, E211Q) in the prion protein gene (PRNP) in inherited prion diseases with Creutzfeldt-Jakob disease phenotype. Hum Mutat. 2000;15(5):482. doi:10.1002/(SICI)1098-1004(200005)15:5<482::AID-HUMU16>3.0.CO;2-1

Modregger J, DiProspero NA, Charles V, Tagle DA, Plomann M. PACSIN 1 interacts with huntingtin and is absent from synaptic varicosities in presymptomatic Huntington's disease brains. Hum Mol Genet. 2002;11(21):25472558. doi:10.1093/hmg/11.21.2547

Schipper HM. Heme oxygenase-1 in Alzheimer disease: a tribute to Moussa Youdim. J Neural Transm (Vienna). 2011;118(3):381-387. doi:10.1007/s00702010-0436-1

Counts SE, Mufson EJ. Regulator of Cell Cycle (RGCC) Expression During the Progression of Alzheimer's Disease.Cell Transplant. 2017;26(4):693-702. doi:10.3727/096368916X694184 
medRxiv preprint doi: https://doi.org/10.1101/2020.12.21.20248688; this version posted December 24, 2020. The copyright holder for this preprint (which was not certified by peer review) is the author/funder, who has granted medRxiv a license to display the preprint in perpetuity. All rights reserved. No reuse allowed without permission.

Olmos-Alonso A, Schetters ST, Sri S, Askew K, Mancuso R, Vargas-Caballero M, Holscher C, Perry VH, Gomez-Nicola D. Pharmacological targeting of CSF1R inhibits microglial proliferation and prevents the progression of Alzheimer's-like pathology. Brain. 2016;139(Pt 3):891-907. doi:10.1093/brain/awv379

Turunen M, Vepsäläinen S, Mäkinen P, Helisalmi S, Haapasalo A, Soininen H, Hiltunen M. No association between high temperature requirement 1 (HTRA1) gene polymorphisms and Alzheimer's disease. Neurobiol Aging. 2011;32(3):547.e7-9. doi:10.1016/j.neurobiolaging.2009.08.010

Baron BW, Pytel P. Expression Pattern of the BCL6 and ITM2B Proteins in Normal Human Brains and in Alzheimer Disease. Appl Immunohistochem Mol Morphol. 2017;25(7):489-496. doi:10.1097/PAI.0000000000000329

Paris D, Ait-Ghezala G, Bachmeier C, Laco G, Beaulieu-Abdelahad D, Lin Y, Jin C, Crawford F, Mullan M. The spleen tyrosine kinase (Syk) regulates Alzheimer amyloid- $\beta$ production and Tau hyperphosphorylation. $J$ Biol Chem. 2014;289(49):33927-33944. doi:10.1074/jbc.M114.608091

Grewal RP, Morgan TE, Finch CE. $\mathrm{ClqB}$ and clusterin mRNA increase in association with neurodegeneration in sporadic amyotrophic lateral sclerosis. Neurosci Lett. 1999;271(1):65-67. doi:10.1016/s0304-3940(99)00496-6

Bernstein HG, Stricker R, Lendeckel U, Bertram I, Dobrowolny H, Steiner J, Bogerts B, Reiser G. Reduced neuronal co-localisation of nardilysin and the putative alpha-secretases ADAM10 and ADAM17 in Alzheimer's disease and Down syndrome brains. Age (Dordr). 2009;31(1):11-25. doi:10.1007/s11357-0089076-X

Koshy B, Miyashita A, St Jean P, Stirnadel H, Kaise T, Rubio JP, Mooser V, Kuwano R, Irizarry MC. Genetic deficiency of plasma lipoprotein-associated phospholipase A2 (PLA2G7 V297F null mutation) and risk of Alzheimer's disease in Japan. J Alzheimers Dis. 2010;21(3):775-780. doi:10.3233/JAD-2010-100513

Chakrabarty P, Li A, Ladd TB, Strickland MR, Koller EJ, Burgess JD, Funk CC, Cruz PE, Allen M, Yaroshenko $M$ et al. TLR5 decoy receptor as a novel antiamyloid therapeutic for Alzheimer's disease. J Exp Med. 2018;215(9):2247-2264. doi:10.1084/jem.20180484 
medRxiv preprint doi: https://doi.org/10.1101/2020.12.21.20248688; this version posted December 24, 2020. The copyright holder for this

Schwam EM, Nicholas T, Chew R, Billing CB, Davidson W, Ambrose D, Altstiel LD. A multicenter, double-blind, placebo-controlled trial of the PDE9A inhibitor, PF-04447943, in Alzheimer's disease. Curr Alzheimer Res. 2014;11(5):413-421.

Keating DJ, Chen C, Pritchard MA. Alzheimer's disease and endocytic dysfunction: clues from the Down syndrome-related proteins, DSCR1 and ITSN1. Ageing Res Rev. 2006;5(4):388-401. doi:10.1016/j.arr.2005.11.001

Yi C, Mei X, Ezan P, Mato S, Matias I, Giaume C, Koulakoff A. Astroglial connexin43 contributes to neuronal suffering in a mouse model of Alzheimer's disease. Cell Death Differ. 2016;23(10):1691-701. doi:10.1038/cdd.2016.63

Chapuis J, Flaig A, Grenier-Boley B, Eysert F, Pottiez V, Deloison G, Vandeputte A, Ayral AM, Mendes T, Desai $S$ et al. Genome-wide, high-content siRNA screening identifies the Alzheimer's genetic risk factor FERMT2 as a major modulator of APP metabolism. Acta Neuropathol. 2017;133(6):955-966. doi:10.1007/s00401-016-1652-z

Zeng F, Zou HQ, Zhou HD, Li J, Wang L, Cao HY, Yi X, Wang X, Liang CR, Wang YR et al. The relationship between single nucleotide polymorphisms of the NTRK2 gene and sporadic Alzheimer's disease in the Chinese Han population. Neurosci Lett. 2013;550:55-59. doi:10.1016/j.neulet.2013.06.061

Shang Z, Lv H, Zhang M, Duan L, Wang S, Li J, Liu G, Ruijie Z, Jiang Y. Genome-wide haplotype association study identify TNFRSF1A, CASP7, LRP1B, CDH1 and TG genes associated with Alzheimer's disease in Caribbean Hispanic individuals. Oncotarget. 2015;6(40):42504-42514. doi:10.18632/oncotarget.6391

Afanador L, Roltsch EA, Holcomb L, Campbell KS, Keeling DA, Zhang Y, Zimmer DB. The Ca2+ sensor S100A1 modulates neuroinflammation, histopathology and Akt activity in the PSAPP Alzheimer's disease mouse model. Cell Calcium. 2014;56(2):68-80. doi:10.1016/j.ceca.2014.05.002

Shao Y, Shaw M, Todd K, Khrestian M, D'Aleo G, Barnard PJ, Zahratka J, Pillai J, $\mathrm{Yu} \mathrm{CE}$, Keene $\mathrm{CD}$ et al. DNA methylation of TOMM40-APOE-APOC2 in Alzheimer's disease. J Hum Genet. 2018;63(4):459-471. doi:10.1038/s10038-0170393-8 
medRxiv preprint doi: https://doi.org/10.1101/2020.12.21.20248688; this version posted December 24, 2020. The copyright holder for this preprint (which was not certified by peer review) is the author/funder, who has granted medRxiv a license to display the preprint in perpetuity. All rights reserved. No reuse allowed without permission.

McAninch EA, Rajan KB, Evans DA, Jo S, Chaker L, Peeters RP, Bennett DA, Mash DC, Bianco AC. A Common DIO2 Polymorphism and Alzheimer Disease Dementia in African and European Americans. J Clin Endocrinol Metab. 2018;103(5):1818-1826. doi:10.1210/jc.2017-01196

Maes OC, Schipper HM, Chong G, Chertkow HM, Wang E. A GSTM3 polymorphism associated with an etiopathogenetic mechanism in Alzheimer disease. Neurobiol Aging. 2010;31(1):34-45. doi:10.1016/j.neurobiolaging.2008.03.007

Deininger MH, Weinschenk T, Meyermann R, Schluesener HJ. The allograft inflammatory factor-1 in Creutzfeldt-Jakob disease brains. Neuropathol Appl Neurobiol. 2003;29(4):389-399.

Kumar R, McClain D, Young R, Carlson GA. Cholesterol transporter ATP-binding cassette A1 (ABCA1) is elevated in prion disease and affects PrPC and PrPSc concentrations in cultured cells. J Gen Virol. 2008;89(Pt 6):1525-32. doi:10.1099/vir.0.83358-0

Rodríguez A, Pérez-Gracia E, Espinosa JC, Pumarola M, Torres JM, Ferrer I. Increased expression of water channel aquaporin 1 and aquaporin 4 in CreutzfeldtJakob disease and in bovine spongiform encephalopathy-infected bovine-PrP transgenic mice. Acta Neuropathol. 2006;112(5):573-585. doi:10.1007/s00401006-0117-1

Kordek R, Nerurkar VR, Liberski PP, Isaacson S, Yanagihara R, Gajdusek DC. Heightened expression of tumor necrosis factor alpha, interleukin 1 alpha, and glial fibrillary acidic protein in experimental Creutzfeldt-Jakob disease in mice. Proc Natl Acad Sci U S A. 1996;93(18):9754-9758. doi:10.1073/pnas.93.18.9754

Claus P, Werner S, Timmer M, Grothe C. Expression of the fibroblast growth factor-2 isoforms and the FGF receptor 1-4 transcripts in the rat model system of Parkinson's disease. Neurosci Lett. 2004;360(3):117-120. doi:10.1016/j.neulet.2004.01.046

Falk T, Zhang S, Sherman SJ. Vascular endothelial growth factor B (VEGF-B) is up-regulated and exogenous VEGF-B is neuroprotective in a culture model of Parkinson's disease. Mol Neurodegener. 2009;4:49. doi:10.1186/1750-1326-4-49 
medRxiv preprint doi: https://doi.org/10.1101/2020.12.21.20248688; this version posted December 24, 2020. The copyright holder for this

Bourque M, Morissette M, Côté M, Soulet D, Di Paolo T. Implication of GPER1 in neuroprotection in a mouse model of Parkinson's disease. Neurobiol Aging. 2013;34(3):887-901. doi:10.1016/j.neurobiolaging.2012.05.022

Kim KS, Marcogliese PC, Yang J, Callaghan SM, Resende V, Abdel-Messih E, Marras C, Visanji NP, Huang J, Schlossmacher MG et al. Regulation of myeloid cell phagocytosis by LRRK2 via WAVE2 complex stabilization is altered in Parkinson's disease. Proc Natl Acad Sci U S A. 2018;115(22):E5164-E5173. doi:10.1073/pnas.1718946115

Mittal S, Bjørnevik K, Im DS, Flierl A, Dong X, Locascio JJ, Abo KM, Long E, Jin $\mathrm{M}, \mathrm{Xu} \mathrm{B}$ et al. $\beta 2$-Adrenoreceptor is a regulator of the $\alpha$-synuclein gene driving risk of Parkinson's disease. Science. 2017;357(6354):891-898. doi:10.1126/science.aaf3934

Okubadejo N, Britton A, Crews C, Akinyemi R, Hardy J, Singleton A, Bras J. Analysis of Nigerians with apparently sporadic Parkinson disease for mutations in LRRK2, PRKN and ATXN3. PLoS One. 2008;3(10):e3421. doi:10.1371/journal.pone.0003421

Stichel CC, Schoenebeck B, Foguet M, Siebertz B, Bader V, Zhu XR, Lübbert H. sgk1, a member of an RNA cluster associated with cell death in a model of Parkinson's disease. Eur J Neurosci. 2005;21(2):301-316. doi:10.1111/j.14609568.2005.03859.x

Lorenzl S, Albers DS, Narr S, Chirichigno J, Beal MF. Expression of MMP-2, MMP-9, and MMP-1 and their endogenous counterregulators TIMP-1 and TIMP-2 in postmortem brain tissue of Parkinson's disease. Exp Neurol. 2002;178(1):13-20. doi:10.1006/exnr.2002.8019

Turic D, Langley K, Williams H, Norton N, Williams NM, Moskvina V, Van den Bree MB, Owen MJ, Thapar A, O'Donovan MC. et al. A family based study implicates solute carrier family 1-member 3 (SLC1A3) gene in attentiondeficit/hyperactivity disorder. Biol Psychiatry. 2005;57(11):1461-1466. doi:10.1016/j.biopsych.2005.03.025 
medRxiv preprint doi: https://doi.org/10.1101/2020.12.21.20248688; this version posted December 24, 2020. The copyright holder for this

Chen X, Wang X, Chen Q, Williamson V, van den Oord E, Maher BS, O'Neill FA, Walsh D, Kendler KS. MEGF10 association with schizophrenia. Biol Psychiatry. 2008;63(5):441-448. doi:10.1016/j.biopsych.2007.11.003

Jia Y, Yu X, Zhang B, Yuan Y, Xu Q, Shen Y, Shen Y. An association study between polymorphisms in three genes of 14-3-3 (tyrosine 3monooxygenase/tryptophan 5-monooxygenase activation protein) family and paranoid schizophrenia in northern Chinese population. Eur Psychiatry. 2004;19(6):377-379. doi:10.1016/j.eurpsy.2004.07.006

Yamamoto A, Suzuki T, Sakaki Y. Isolation of hNap1BP which interacts with human Nap1 (NCKAP1) whose expression is down-regulated in Alzheimer's disease. Gene. 2001;271(2):159-169. doi:10.1016/s0378-1119(01)00521-2

Bill BR, Lowe JK, Dybuncio CT, Fogel BL. Orchestration of neurodevelopmental programs by RBFOX1: implications for autism spectrum disorder. Int Rev Neurobiol. 2013;113:251-267. doi:10.1016/B978-0-12-418700-9.00008-3

Wilhelmus MM, Boelens WC, Otte-Höller I, Kamps B, Kusters B, Maat-Schieman ML, de Waal RM, Verbeek MM. Small heat shock protein HspB8: its distribution in Alzheimer's disease brains and its inhibition of amyloid-beta protein aggregation and cerebrovascular amyloid-beta toxicity. Acta Neuropathol. 2006;111(2):139149. doi:10.1007/s00401-005-0030-z

Seidel K, Vinet J, Dunnen WF, Brunt ER, Meister M, Boncoraglio A, Zijlstra MP, Boddeke HW, Rüb U, Kampinga HH et al. The HSPB8-BAG3 chaperone complex is upregulated in astrocytes in the human brain affected by protein aggregation diseases. Neuropathol Appl Neurobiol. 2012;38(1):39-53. doi:10.1111/j.13652990.2011.01198.x 
medRxiv preprint doi: https://doi.org/10.1101/2020.12.21.20248688; this version posted December 24, 2020. The copyright holder for this preprint (which was not certified by peer review) is the author/funder, who has granted medRxiv a license to display the preprint in perpetuity.

All rights reserved. No reuse allowed without permission.

\section{Tables}

Table 1 The statistical metrics for key differentially expressed genes (DEGs)

\begin{tabular}{|c|c|c|c|c|c|c|c|}
\hline Illumina Id & Gene Symbol & $\log \mathrm{FC}$ & P Value & $\begin{array}{l}\text { Adj. P } \\
\text { Value }\end{array}$ & t value & Regulation & Gene Name \\
\hline ILMN_1803036 & TARBP1 & 0.862546 & 7.81E-09 & 0.000122 & 9.215789 & Up & TAR (HIV-1) RNA binding protein 1 \\
\hline ILMN_1756701 & DHRS11 & 0.863093 & $3.8 \mathrm{E}-07$ & 0.000337 & 7.250194 & Up & dehydrogenase/reductase 11 \\
\hline ILMN_2204754 & TMX4 & 0.934932 & $6.8 \mathrm{E}-07$ & 0.000381 & 6.976256 & Up & thioredoxin related transmembrane protein 4 \\
\hline ILMN_1713491 & VAMP2 & 1.205196 & $8.98 \mathrm{E}-07$ & 0.000408 & 6.847422 & Up & vesicle associated membrane protein 2 \\
\hline ILMN_1814787 & ICA1 & 1.096951 & 9E-07 & 0.000408 & 6.846489 & Up & islet cell autoantigen 1 \\
\hline ILMN_1771652 & BAIAP2L2 & 0.946928 & $3.01 \mathrm{E}-06$ & 0.000587 & 6.296104 & Up & BAI1 associated protein 2 like 2 \\
\hline ILMN_1674661 & CIRBP & 1.937232 & $3.31 \mathrm{E}-06$ & 0.000602 & 6.253559 & Up & cold inducible RNA binding protein \\
\hline ILMN_2365569 & ICA1 & 0.907275 & $3.38 \mathrm{E}-06$ & 0.000609 & 6.245363 & Up & islet cell autoantigen 1 \\
\hline ILMN_1682326 & PCP4 & 2.050131 & $3.42 \mathrm{E}-06$ & 0.000609 & 6.239412 & Up & Purkinje cell protein 4 \\
\hline ILMN_1779742 & GABRD & 0.922308 & $3.47 \mathrm{E}-06$ & 0.000609 & 6.232455 & Up & gamma-aminobutyric acid type A receptor delta subunit \\
\hline ILMN_1808765 & ZNF25 & 1.182797 & $3.52 \mathrm{E}-06$ & 0.000609 & 6.226058 & Up & zinc finger protein 25 \\
\hline ILMN_1652540 & RELL2 & 1.110282 & $3.68 \mathrm{E}-06$ & 0.000613 & 6.206913 & Up & RELT like 2 \\
\hline ILMN_1719998 & STRBP & 0.992143 & 4.01E-06 & 0.000613 & 6.168852 & Up & spermatid perinuclear RNA binding protein \\
\hline ILMN_1779241 & CRYM & 1.258922 & 4.14E-06 & 0.000613 & 6.154647 & Up & crystallin mu \\
\hline ILMN_1727805 & SYNGR1 & 0.949612 & $4.22 \mathrm{E}-06$ & 0.000613 & 6.145733 & Up & synaptogyrin 1 \\
\hline ILMN_1812297 & CYP26B1 & 1.695928 & 4.35E-06 & 0.000613 & 6.132594 & Up & cytochrome P450 family 26 subfamily B member 1 \\
\hline ILMN_2345739 & CAPRIN2 & 0.999502 & $5.33 \mathrm{E}-06$ & 0.000666 & 6.042169 & Up & caprin family member 2 \\
\hline ILMN_1769911 & SLC38A1 & 1.016462 & $5.63 \mathrm{E}-06$ & 0.000667 & 6.018074 & Up & solute carrier family 38 member 1 \\
\hline ILMN_1763412 & SPHKAP & 1.196793 & $6.22 \mathrm{E}-06$ & 0.000693 & 5.974505 & Up & SPHK1 interactor, AKAP domain containing \\
\hline ILMN_1712352 & DOCK3 & 1.178133 & $6.68 \mathrm{E}-06$ & 0.000705 & 5.942612 & Up & dedicator of cytokinesis 3 \\
\hline ILMN_2354478 & CYFIP2 & 1.308629 & $6.75 \mathrm{E}-06$ & 0.000705 & 5.938463 & Up & cytoplasmic FMR1 interacting protein 2 \\
\hline ILMN_2381938 & ATP2B2 & 1.659403 & $6.76 \mathrm{E}-06$ & 0.000705 & 5.937753 & Up & ATPase plasma membrane $\mathrm{Ca} 2+$ transporting 2 \\
\hline ILMN_1695947 & SCN4B & 0.856007 & $7.19 \mathrm{E}-06$ & 0.000722 & 5.910291 & Up & sodium voltage-gated channel beta subunit 4 \\
\hline ILMN_1682938 & ARF3 & 0.863489 & $7.26 \mathrm{E}-06$ & 0.000722 & 5.90645 & Up & ADP ribosylation factor 3 \\
\hline ILMN_1758128 & CYGB & 0.966147 & $7.55 \mathrm{E}-06$ & 0.000729 & 5.888887 & Up & cytoglobin \\
\hline ILMN_1735553 & MAP3K9 & 0.88995 & $8.15 \mathrm{E}-06$ & 0.000757 & 5.855754 & Up & mitogen-activated protein kinase kinasekinase 9 \\
\hline ILMN_2400947 & CELF2 & 0.998713 & $8.61 \mathrm{E}-06$ & 0.000777 & 5.831371 & Up & CUGBP Elav-like family member 2 \\
\hline ILMN_1793651 & UBE2N & 0.922853 & $8.77 \mathrm{E}-06$ & 0.000781 & 5.823227 & Up & ubiquitin conjugating enzyme $\mathrm{E} 2 \mathrm{~N}$ \\
\hline ILMN_1730818 & MIR7-3HG & 0.98763 & $8.82 \mathrm{E}-06$ & 0.000781 & 5.820718 & Up & MIR7-3 host gene \\
\hline ILMN_1662470 & FAM241B & 0.921613 & $9.1 \mathrm{E}-06$ & 0.000785 & 5.807224 & Up & family with sequence similarity 241 member B \\
\hline ILMN_1764780 & SVOP & 1.731461 & $9.43 \mathrm{E}-06$ & 0.000789 & 5.791618 & Up & $\mathrm{SV} 2$ related protein \\
\hline ILMN_1795561 & CAMK1D & 1.083349 & $9.51 \mathrm{E}-06$ & 0.000789 & 5.788122 & Up & calcium/calmodulin dependent protein kinase ID \\
\hline ILMN_1657554 & TSPYL2 & 1.050072 & $9.51 \mathrm{E}-06$ & 0.000789 & 5.787888 & Up & TSPY like 2 \\
\hline ILMN_1692398 & CNTNAP1 & 1.130697 & $1.03 \mathrm{E}-05$ & 0.00081 & 5.7551 & Up & contactin associated protein 1 \\
\hline ILMN_1802223 & ATP2B2 & 1.235419 & $1.04 \mathrm{E}-05$ & 0.00081 & 5.749995 & Up & ATPase plasma membrane $\mathrm{Ca} 2+$ transporting 2 \\
\hline ILMN_1784783 & NME5 & 0.935549 & $1.11 \mathrm{E}-05$ & 0.00082 & 5.720686 & Up & NME/NM23 family member 5 \\
\hline
\end{tabular}




\begin{tabular}{|c|c|c|c|c|c|}
\hline ILMN_1723837 & SLC6A17 & 1.33733 & $1.15 \mathrm{E}-05$ & 0.000837 & 5.706401 \\
\hline ILMN_1680738 & NREP & 1.304269 & $1.16 \mathrm{E}-05$ & 0.000847 & 5.700352 \\
\hline ILMN_1805039 & DPP10 & 0.939222 & $1.23 \mathrm{E}-05$ & 0.000867 & 5.674692 \\
\hline ILMN_2397646 & GPM6A & 1.37146 & $1.24 \mathrm{E}-05$ & 0.000867 & 5.673776 \\
\hline ILMN_1736015 & JADE1 & 0.949637 & $1.26 \mathrm{E}-05$ & 0.000877 & 5.665905 \\
\hline ILMN_1753345 & SCAMP5 & 1.137935 & $1.34 \mathrm{E}-05$ & 0.000894 & 5.637955 \\
\hline ILMN_1779428 & CCDC184 & 1.321623 & $1.35 \mathrm{E}-05$ & 0.000894 & 5.635181 \\
\hline ILMN_2414878 & STXBP1 & 2.276537 & $1.39 \mathrm{E}-05$ & 0.000902 & 5.621837 \\
\hline ILMN_1654541 & ATP6V1G2 & 1.571122 & $1.44 \mathrm{E}-05$ & 0.000926 & 5.608303 \\
\hline ILMN_1703408 & FZD3 & 0.958702 & $1.5 \mathrm{E}-05$ & 0.000936 & 5.59104 \\
\hline ILMN_1734021 & CLSTN3 & 1.545043 & $1.54 \mathrm{E}-05$ & 0.000949 & 5.578818 \\
\hline ILMN_1732066 & CKMT1A & 1.325128 & $1.57 \mathrm{E}-05$ & 0.000953 & 5.571652 \\
\hline ILMN_1744937 & PTPRM & 0.917724 & $1.62 \mathrm{E}-05$ & 0.000962 & 5.556377 \\
\hline ILMN_1722559 & NEUROD6 & 1.043615 & $1.64 \mathrm{E}-05$ & 0.000962 & 5.550915 \\
\hline ILMN_1728844 & PTPRN2 & 0.935214 & $1.72 \mathrm{E}-05$ & 0.000987 & 5.531362 \\
\hline ILMN_2165463 & HAPLN4 & 1.426121 & $1.73 \mathrm{E}-05$ & 0.000994 & 5.527401 \\
\hline ILMN_1701483 & SYP & 1.850749 & $1.75 \mathrm{E}-05$ & 0.001001 & 5.522234 \\
\hline ILMN_1803818 & NMNAT2 & 1.622559 & $1.85 \mathrm{E}-05$ & 0.001033 & 5.499488 \\
\hline ILMN_2415189 & ATP1A1 & 1.223192 & $1.93 \mathrm{E}-05$ & 0.001053 & 5.481202 \\
\hline ILMN_1720844 & SSX2IP & 0.871649 & $1.95 \mathrm{E}-05$ & 0.001053 & 5.47711 \\
\hline ILMN_1772627 & NSG1 & 1.612518 & $2 \mathrm{E}-05$ & 0.00106 & 5.464876 \\
\hline ILMN_1785141 & MICAL2 & 1.139667 & $2.04 \mathrm{E}-05$ & 0.00107 & 5.45801 \\
\hline ILMN_1721605 & SMYD2 & 1.16347 & $2.1 \mathrm{E}-05$ & 0.001083 & 5.444879 \\
\hline ILMN_1754727 & GPRASP2 & 1.424101 & $2.11 \mathrm{E}-05$ & 0.001084 & 5.442489 \\
\hline ILMN_1801703 & CPLX1 & 1.453918 & $2.17 \mathrm{E}-05$ & 0.0011 & 5.430795 \\
\hline ILMN_1765796 & ENO2 & 1.299932 & $2.2 \mathrm{E}-05$ & 0.0011 & 5.424636 \\
\hline ILMN_1712506 & DPP6 & 1.033487 & $2.2 \mathrm{E}-05$ & 0.0011 & 5.424246 \\
\hline ILMN_1676998 & $\mathrm{SCN} 2 \mathrm{~B}$ & 1.546344 & $2.24 \mathrm{E}-05$ & 0.0011 & 5.417596 \\
\hline ILMN_1796673 & RIT2 & 0.914308 & $2.26 \mathrm{E}-05$ & 0.001102 & 5.413704 \\
\hline ILMN_1672121 & CCDC184 & 1.246942 & $2.28 \mathrm{E}-05$ & 0.001107 & 5.408925 \\
\hline ILMN_1664537 & USP11 & 0.88041 & $2.31 \mathrm{E}-05$ & 0.001115 & 5.403764 \\
\hline ILMN_1684143 & NPTXR & 0.953438 & $2.32 \mathrm{E}-05$ & 0.001115 & 5.402735 \\
\hline ILMN_1791726 & TUBB3 & 1.350431 & $2.35 \mathrm{E}-05$ & 0.001115 & 5.397323 \\
\hline ILMN_1717803 & CDK5R2 & 1.050159 & $2.38 \mathrm{E}-05$ & 0.001117 & 5.391108 \\
\hline ILMN_1700628 & DDX24 & 0.965537 & $2.39 \mathrm{E}-05$ & 0.001121 & 5.388703 \\
\hline ILMN_1717799 & PRKCE & 1.392944 & $2.42 \mathrm{E}-05$ & 0.001124 & 5.383748 \\
\hline ILMN_2038775 & TUBB2A & 1.142039 & $2.52 \mathrm{E}-05$ & 0.001153 & 5.36694 \\
\hline ILMN_2044813 & TUBB2A & 1.13073 & $2.53 \mathrm{E}-05$ & 0.001153 & 5.365452 \\
\hline ILMN_1653200 & SLC22A17 & 0.908235 & $2.62 \mathrm{E}-05$ & 0.001165 & 5.350312 \\
\hline ILMN_2359168 & RBFOX1 & 1.348472 & $2.74 \mathrm{E}-05$ & 0.001195 & 5.331188 \\
\hline ILMN_1728747 & STXBP1 & 1.562887 & $2.74 \mathrm{E}-05$ & 0.001195 & 5.330369 \\
\hline LMN_1760088 & CCKBR & 1.114844 & $2.81 \mathrm{E}-05$ & 0.001211 & 5.320566 \\
\hline
\end{tabular}

solute carrier family 6 member 17 neuronal regeneration related protein dipeptidyl peptidase like 10 glycoprotein M6A jade family PHD finger 1 secretory carrier membrane protein 5 coiled-coil domain containing 184 syntaxin binding protein 1 ATPase $\mathrm{H}+$ transporting V1 subunit $\mathrm{G} 2$ frizzled class receptor 3 calsyntenin 3

creatine kinase, mitochondrial 1A protein tyrosine phosphatase, receptor type $\mathrm{M}$ neuronal differentiation 6 protein tyrosine phosphatase, receptor type N2 hyaluronan and proteoglycan link protein 4 synaptophysin

nicotinamide nucleotide adenylyltransferase 2

ATPase $\mathrm{Na}+\mathrm{K}+$ transporting subunit alpha 1

SSX family member 2 interacting protein

neuronal vesicle trafficking associated 1 microtubule associated monooxygenase, calponin and LIM domain containing 2

SET and MYND domain containing 2

$\mathrm{G}$ protein-coupled receptor associated sorting protein 2 complexin 1 enolase 2 dipeptidyl peptidase like 6 sodium voltage-gated channel beta subunit 2 Ras like without CAAX 2 coiled-coil domain containing 184 ubiquitin specific peptidase 11 neuronal pentraxin receptor tubulin beta 3 class III

cyclin dependent kinase 5 regulatory subunit 2 DEAD-box helicase 24 protein kinase $\mathrm{C}$ epsilon tubulin beta $2 \mathrm{~A}$ class IIa tubulin beta $2 \mathrm{~A}$ class IIa

solute carrier family 22 member 17

RNA binding fox-1 homolog 1 syntaxin binding protein 1 cholecystokinin B receptor 


\begin{tabular}{|c|c|c|c|c|c|c|c|}
\hline ILMN_1685496 & RGS7 & 1.71146 & $2.83 \mathrm{E}-05$ & 0.001211 & 5.317251 & Up & regulator of $\mathrm{G}$ protein signaling 7 \\
\hline ILMN_1658289 & WDR54 & 1.138994 & $2.84 \mathrm{E}-05$ & 0.001212 & 5.315345 & $\mathrm{Up}$ & WD repeat domain 54 \\
\hline ILMN_2195462 & C1QTNF4 & 1.223524 & $2.92 \mathrm{E}-05$ & 0.001236 & 5.302989 & $\mathrm{Up}$ & $\mathrm{C} 1 \mathrm{q}$ and $\mathrm{TNF}$ related 4 \\
\hline ILMN_1786379 & SYNGR3 & 1.432497 & $2.95 \mathrm{E}-05$ & 0.001242 & 5.298854 & Up & synaptogyrin 3 \\
\hline ILMN_1763750 & NPTX1 & 1.361965 & $2.98 \mathrm{E}-05$ & 0.001246 & 5.295223 & $\mathrm{Up}$ & neuronal pentraxin 1 \\
\hline ILMN_1701637 & SULT4A1 & 1.825606 & $2.98 \mathrm{E}-05$ & 0.001246 & 5.294821 & $\mathrm{Up}$ & sulfotransferase family $4 \mathrm{~A}$ member 1 \\
\hline ILMN_1806147 & GNG3 & 1.056058 & 3.04E-05 & 0.001251 & 5.287106 & Up & G protein subunit gamma 3 \\
\hline ILMN_1772645 & AGK & 0.908287 & $3.05 \mathrm{E}-05$ & 0.001252 & 5.28498 & Up & acylglycerol kinase \\
\hline ILMN_1796962 & PPP3R1 & 1.221939 & 3.1E-05 & 0.001261 & 5.278764 & Up & protein phosphatase 3 regulatory subunit $\mathrm{B}$, alpha \\
\hline ILMN_1663444 & LIN7B & 1.00565 & $3.12 \mathrm{E}-05$ & 0.001265 & 5.275107 & Up & $\begin{array}{l}\text { lin-7 homolog B, crumbs cell polarity complex component } \\
\text { HECT and RLD domain containing E3 ubiquitin protein }\end{array}$ \\
\hline ILMN_1786211 & HERC1 & 1.04834 & $3.19 \mathrm{E}-05$ & 0.00128 & 5.26529 & Up & ligase family member 1 \\
\hline ILMN_2221808 & DGCR5 & 1.004588 & $3.26 \mathrm{E}-05$ & 0.001287 & 5.256214 & Up & DiGeorge syndrome critical region gene 5 \\
\hline ILMN_2151281 & GABARAPL1 & 1.229594 & $3.44 \mathrm{E}-05$ & 0.001312 & 5.233168 & Up & GABA type A receptor associated protein like 1 \\
\hline ILMN_1808095 & RNF41 & 1.041425 & 3.55E-05 & 0.001336 & 5.220184 & Up & ring finger protein 41 \\
\hline ILMN_2120210 & RCAN2 & 1.430712 & 3.63E-05 & 0.001342 & 5.211143 & Up & regulator of calcineurin 2 \\
\hline ILMN_1683044 & PPP1R2 & 0.857977 & $3.63 \mathrm{E}-05$ & 0.001342 & 5.210627 & Up & protein phosphatase 1 regulatory inhibitor subunit 2 \\
\hline ILMN_1779343 & $\mathrm{SNCB}$ & 2.22442 & 3.74E-05 & 0.001362 & 5.197702 & Up & synuclein beta \\
\hline ILMN_2215989 & NEFM & 2.490849 & $3.75 \mathrm{E}-05$ & 0.001362 & 5.196827 & Up & neurofilament medium \\
\hline ILMN_1729165 & TCEAL6 & 1.347129 & $3.95 \mathrm{E}-05$ & 0.001411 & 5.17479 & Up & transcription elongation factor A like 6 \\
\hline ILMN_1809613 & NGEF & 1.29077 & 3.97E-05 & 0.001416 & 5.172601 & $\mathrm{Up}$ & neuronal guanine nucleotide exchange factor \\
\hline ILMN_1731783 & ATP1A1 & 1.590389 & 3.98E-05 & 0.001416 & 5.171812 & $\mathrm{Up}$ & ATPase $\mathrm{Na}+\mathrm{K}+$ transporting subunit alpha 1 \\
\hline ILMN_1658576 & PTPRN & 1.23347 & 4.14E-05 & 0.001444 & 5.15524 & Up & protein tyrosine phosphatase, receptor type $\mathrm{N}$ \\
\hline ILMN_1693481 & ATP2B3 & 1.00926 & 4.14E-05 & 0.001444 & 5.154901 & Up & ATPase plasma membrane $\mathrm{Ca} 2+$ transporting 3 \\
\hline ILMN_1763344 & ADCYAP1 & 1.015132 & 4.14E-05 & 0.001444 & 5.15476 & Up & adenylatecyclase activating polypeptide 1 \\
\hline ILMN_2120555 & ADCY1 & 1.636339 & 4.15E-05 & 0.001444 & 5.153623 & Up & adenylatecyclase 1 \\
\hline ILMN_1765966 & CHGB & 2.106015 & $4.26 \mathrm{E}-05$ & 0.001459 & 5.142884 & Up & chromogranin B \\
\hline ILMN_1760160 & STX1A & 1.787104 & 4.27E-05 & 0.001459 & 5.141842 & $\mathrm{Up}$ & syntaxin $1 \mathrm{~A}$ \\
\hline ILMN_2319326 & ADARB1 & 0.861643 & 4.4E-05 & 0.001483 & 5.128896 & $\mathrm{Up}$ & adenosine deaminase, RNA specific B1 \\
\hline ILMN_1716563 & PRKCB & 1.776401 & 4.42E-05 & 0.001483 & 5.127035 & Up & protein kinase $\mathrm{C}$ beta \\
\hline ILMN_1762531 & FGF9 & 1.042022 & 4.42E-05 & 0.001483 & 5.126907 & Up & fibroblast growth factor 9 \\
\hline ILMN_1800381 & GNAO1 & 1.29281 & 4.54E-05 & 0.001506 & 5.115838 & Up & G protein subunit alpha o1 \\
\hline ILMN_1806908 & PRKCB & 1.511486 & 4.7E-05 & 0.001532 & 5.10128 & Up & protein kinase $\mathrm{C}$ beta \\
\hline ILMN_1715927 & VSTM2L & 1.084737 & 4.73E-05 & 0.001534 & 5.097857 & Up & $\mathrm{V}$-set and transmembrane domain containing 2 like \\
\hline ILMN_2199768 & SLITRK4 & 1.258524 & 4.74E-05 & 0.001534 & 5.097762 & $\mathrm{Up}$ & SLIT and NTRK like family member 4 \\
\hline ILMN_1785202 & STAT4 & 1.640651 & $5.06 \mathrm{E}-05$ & 0.001572 & 5.069645 & Up & signal transducer and activator of transcription 4 \\
\hline ILMN_1814316 & RBFOX1 & 1.382443 & $5.07 \mathrm{E}-05$ & 0.001572 & 5.06842 & Up & RNA binding fox- 1 homolog 1 \\
\hline ILMN_2342554 & TAGLN3 & 2.095976 & $5.34 \mathrm{E}-05$ & 0.001625 & 5.046396 & Up & transgelin 3 \\
\hline ILMN_1687821 & C16orf45 & 1.077658 & $5.37 \mathrm{E}-05$ & 0.001631 & 5.044189 & Up & chromosome 16 open reading frame 45 \\
\hline ILMN_1788053 & SLC25A12 & 1.247596 & $5.39 \mathrm{E}-05$ & 0.001632 & 5.043036 & Up & solute carrier family 25 member 12 \\
\hline ILMN_1655177 & PI4KA & 0.899499 & $5.45 \mathrm{E}-05$ & 0.001639 & 5.038219 & Up & phosphatidylinositol 4-kinase alpha \\
\hline ILMN_1767662 & CERS6 & 1.179006 & $5.46 \mathrm{E}-05$ & 0.001639 & 5.03744 & Up & ceramide synthase 6 \\
\hline ILMN_1673152 & NRXN1 & 0.871951 & 5.53E-05 & 0.001654 & 5.032187 & $\mathrm{Up}$ & neurexin 1 \\
\hline
\end{tabular}


medRxiv preprint doi: https://doi.org/10.1101/2020.12.21.20248688; this version posted December 24, 2020. The copyright holder for this preprint (which was not certified by peer review) is the author/funder, who has granted medRxiv a license to display the preprint in perpetuity. All rights reserved. No reuse allowed without permission.

\begin{tabular}{|c|c|c|c|c|c|c|c|}
\hline ILMN_1794200 & $\mathrm{KCNJ} 12$ & 0.908552 & $5.77 \mathrm{E}-05$ & 0.001692 & 5.014264 & Up & \\
\hline ILMN_1670000 & DCAF6 & 1.163643 & $5.79 \mathrm{E}-05$ & 0.001694 & 5.012302 & Up & DDB1 and CUL4 associated factor 6 \\
\hline ILMN_2332250 & ACOT7 & 1.61114 & $5.86 \mathrm{E}-05$ & 0.001705 & 5.007401 & Up & acyl-CoA thioesterase 7 \\
\hline ILMN_1679256 & AP3B2 & 1.085251 & $5.89 \mathrm{E}-05$ & 0.001709 & 5.005566 & Up & adaptor related protein complex 3 subunit beta 2 \\
\hline ILMN_1751020 & PACSIN1 & 1.253138 & $5.92 \mathrm{E}-05$ & 0.001709 & 5.002919 & Up & protein kinase $\mathrm{C}$ and casein kinase substrate in neurons 1 \\
\hline ILMN_2408430 & LARGE1 & 1.142725 & $5.93 \mathrm{E}-05$ & 0.001709 & 5.002365 & Up & LARGE xylosyl- and glucuronyltransferase 1 \\
\hline ILMN_1685699 & PRSS3 & 1.064411 & $5.94 \mathrm{E}-05$ & 0.001709 & 5.001544 & Up & serine protease 3 \\
\hline ILMN_1767365 & PAK1 & 1.603493 & $5.99 \mathrm{E}-05$ & 0.001711 & 4.997957 & Up & p21 (RAC1) activated kinase 1 \\
\hline ILMN_1759613 & PNMA6A & 1.025044 & $6.03 \mathrm{E}-05$ & 0.001713 & 4.995636 & Up & PNMA family member $6 \mathrm{~A}$ \\
\hline ILMN_2241750 & $\begin{array}{c}\text { KALRN } \\
\text { ATP6V1G2- }\end{array}$ & 0.921739 & $6.12 \mathrm{E}-05$ & 0.00172 & 4.988947 & $\mathrm{Up}$ & kalirinRhoGEF kinase \\
\hline ILMN_1674778 & DDX39B & 1.358803 & $6.23 \mathrm{E}-05$ & 0.00174 & 4.981273 & Up & ATP6V1G2-DDX39B readthrough (NMD candidate) \\
\hline ILMN_2377900 & MAP1B & 1.551639 & $6.25 \mathrm{E}-05$ & 0.001741 & 4.979938 & Up & microtubule associated protein $1 \mathrm{~B}$ \\
\hline ILMN_1677439 & GLS2 & 0.985317 & $6.28 \mathrm{E}-05$ & 0.001741 & 4.978289 & Up & glutaminase 2 \\
\hline ILMN_1734695 & MAP4 & 1.805336 & $6.39 \mathrm{E}-05$ & 0.001747 & 4.970938 & Up & microtubule associated protein 4 \\
\hline ILMN_1690397 & DYNC1I1 & 1.784178 & $6.42 \mathrm{E}-05$ & 0.001751 & 4.968675 & Up & dynein cytoplasmic 1 intermediate chain 1 \\
\hline ILMN_2281786 & RTN1 & 2.860193 & $6.44 \mathrm{E}-05$ & 0.001751 & 4.967598 & Up & reticulon 1 \\
\hline ILMN_1770758 & AKAP6 & 0.91749 & $6.45 \mathrm{E}-05$ & 0.001753 & 4.966713 & Up & A-kinase anchoring protein 6 \\
\hline ILMN_1770938 & PAK5 & 1.103874 & $6.48 \mathrm{E}-05$ & 0.001753 & 4.964722 & Up & p21 (RAC1) activated kinase 5 \\
\hline ILMN_1801090 & KRT222 & 1.307607 & $6.65 \mathrm{E}-05$ & 0.001764 & 4.953809 & Up & keratin 222 \\
\hline ILMN_1758825 & ABLIM2 & 1.327023 & $6.74 \mathrm{E}-05$ & 0.001779 & 4.948065 & Up & actin binding LIM protein family member 2 \\
\hline ILMN_1751689 & CHRM1 & 1.184176 & $6.79 \mathrm{E}-05$ & 0.001784 & 4.945433 & Up & cholinergic receptor muscarinic 1 \\
\hline ILMN_1679984 & $\mathrm{ZCCHC} 12$ & 1.664142 & $6.92 \mathrm{E}-05$ & 0.001803 & 4.937304 & Up & zinc finger CCHC-type containing 12 \\
\hline ILMN_2412294 & GNB5 & 1.038777 & $6.97 \mathrm{E}-05$ & 0.001807 & 4.934222 & Up & G protein subunit beta 5 \\
\hline ILMN_1657796 & STMN1 & 1.226101 & 7.01E-05 & 0.001811 & 4.931623 & Up & stathmin 1 \\
\hline ILMN_1716507 & EPB41L1 & 1.151569 & 7.03E-05 & 0.001812 & 4.930356 & Up & erythrocyte membrane protein band 4.1 like 1 \\
\hline ILMN_1802633 & GABRA1 & 1.217609 & 7.11E-05 & 0.00182 & 4.925449 & Up & gamma-aminobutyric acid type A receptor alpha1 subunit \\
\hline ILMN_2180371 & FAM216A & 1.008591 & $7.18 \mathrm{E}-05$ & 0.001824 & 4.921628 & Up & family with sequence similarity 216 member A \\
\hline ILMN_1739812 & PRKCG & 0.959013 & $7.25 \mathrm{E}-05$ & 0.001834 & 4.917253 & Up & protein kinase $\mathrm{C}$ gamma \\
\hline ILMN_1733831 & PGBD5 & 0.968327 & 7.39E-05 & 0.00185 & 4.909418 & Up & piggyBac transposable element derived 5 \\
\hline ILMN_2165354 & DCLK1 & 2.138468 & 7.43E-05 & 0.001852 & 4.90726 & Up & doublecortin like kinase 1 \\
\hline ILMN_2104295 & TMEM178A & 1.366287 & 7.44E-05 & 0.001852 & 4.906361 & Up & transmembrane protein $178 \mathrm{~A}$ \\
\hline ILMN_1756928 & RTN1 & 2.206973 & 7.45E-05 & 0.001852 & 4.905889 & Up & reticulon 1 \\
\hline ILMN_1672022 & EPHA4 & 1.59297 & 7.6E-05 & 0.00187 & 4.897643 & Up & EPH receptor A4 \\
\hline ILMN_1737611 & VAMP1 & 1.668208 & 7.69E-05 & 0.00189 & 4.892354 & Up & vesicle associated membrane protein 1 \\
\hline ILMN_2175317 & CYP4X1 & 1.140455 & 7.73E-05 & 0.001897 & 4.890179 & Up & cytochrome P450 family 4 subfamily X member 1 \\
\hline ILMN_1675797 & EPDR1 & 1.424979 & 7.83E-05 & 0.001916 & 4.884851 & Up & ependymin related 1 \\
\hline ILMN_1789505 & ITPR1 & 1.51934 & $8.12 \mathrm{E}-05$ & 0.001955 & 4.869687 & Up & inositol 1,4,5-trisphosphate receptor type 1 \\
\hline ILMN_1798485 & ATP6V1E1 & 0.872992 & $8.29 \mathrm{E}-05$ & 0.001983 & 4.86103 & Up & ATPase $\mathrm{H}+$ transporting V1 subunit E1 \\
\hline ILMN_1779014 & TSPYL1 & 1.542033 & $8.65 \mathrm{E}-05$ & 0.002024 & 4.843112 & $\mathrm{Up}$ & TSPY like 1 \\
\hline ILMN_1693233 & KIAA0513 & 1.134577 & $8.7 \mathrm{E}-05$ & 0.002028 & 4.840586 & Up & KIAA0513 \\
\hline ILMN_2126038 & STMN2 & 1.663457 & $8.71 \mathrm{E}-05$ & 0.002028 & 4.839929 & Up & stathmin 2 \\
\hline ILMN_1796417 & ASNS & 0.895524 & $8.73 \mathrm{E}-05$ & 0.002028 & 4.839186 & $\mathrm{Up}$ & asparagine synthetase (glutamine-hydrolyzing) \\
\hline
\end{tabular}




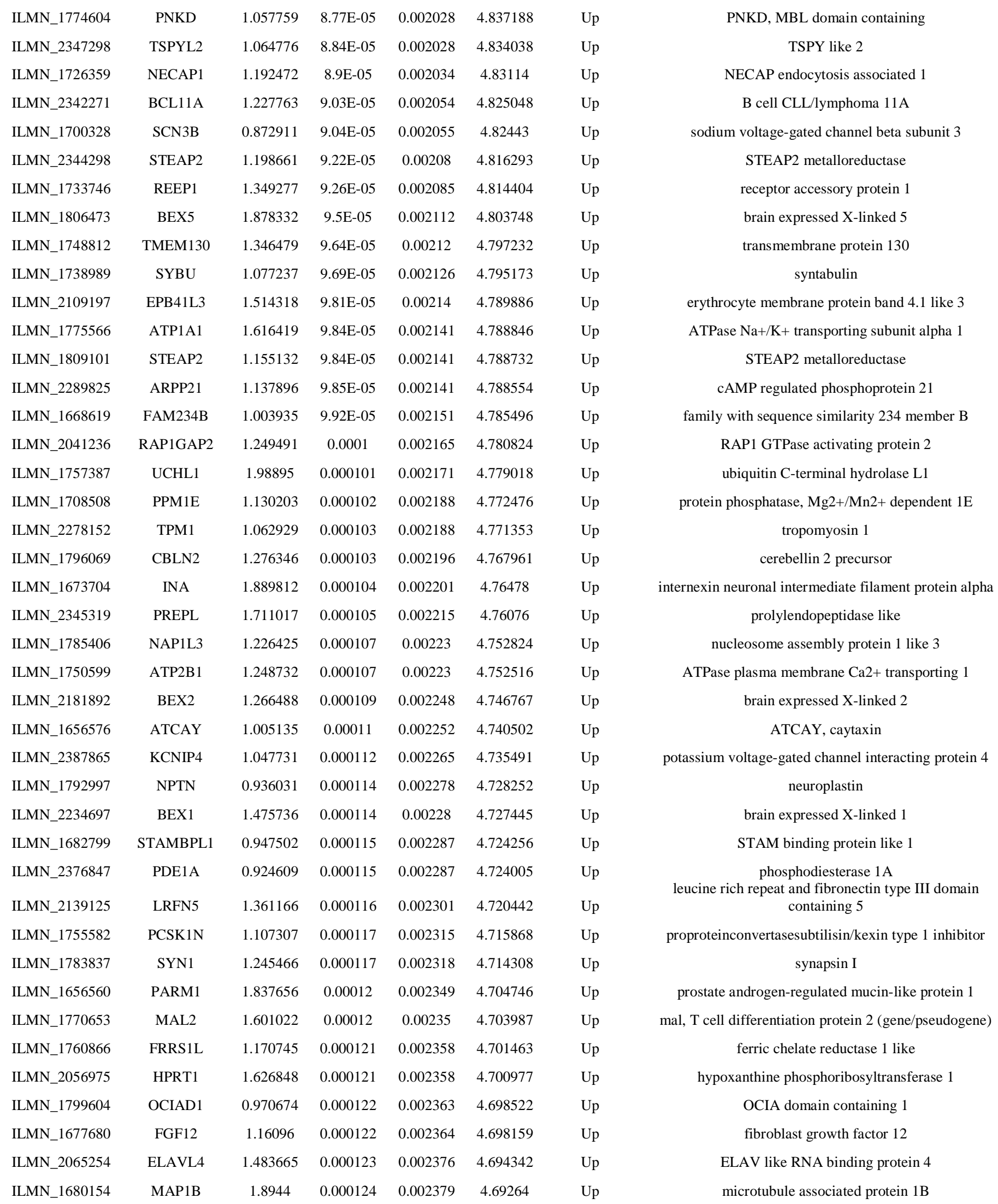




\begin{tabular}{|c|c|c|c|c|c|c|c|}
\hline ILMN_2406106 & CACNB2 & 1.029685 & 0.000125 & 0.002387 & 4.689352 & Up & calcium voltage-gated channel auxiliary subunit beta 2 \\
\hline ILMN_1759117 & $\mathrm{XK}$ & 1.015353 & 0.000125 & 0.002387 & 4.688738 & Up & $\mathrm{X}$-linked Kx blood group \\
\hline ILMN_1745817 & NELL1 & 1.650308 & 0.000128 & 0.002433 & 4.677612 & Up & neural EGFL like 1 \\
\hline ILMN_1666385 & CALM3 & 0.908609 & 0.000132 & 0.002471 & 4.664155 & Up & calmodulin 3 \\
\hline ILMN_1653762 & NRSN1 & 1.489678 & 0.000137 & 0.002515 & 4.650257 & Up & neurensin 1 \\
\hline ILMN_1717477 & PSD3 & 1.235456 & 0.000137 & 0.002515 & 4.649539 & Up & pleckstrin and Sec7 domain containing 3 \\
\hline ILMN_2408080 & SNAP25 & 2.729178 & 0.000137 & 0.002515 & 4.648781 & Up & synaptosome associated protein 25 \\
\hline ILMN_2322446 & PABPC1L2B & 0.981356 & 0.000138 & 0.002516 & 4.646219 & Up & poly(A) binding protein cytoplasmic 1 like $2 \mathrm{~B}$ \\
\hline ILMN_2100689 & MAP2K4 & 1.174395 & 0.00014 & 0.002538 & 4.641062 & Up & mitogen-activated protein kinase kinase 4 \\
\hline ILMN_1745994 & GAS7 & 0.868175 & 0.000141 & 0.002556 & 4.636857 & Up & growth arrest specific 7 \\
\hline ILMN_1705663 & DMXL2 & 0.858505 & 0.000142 & 0.002558 & 4.636103 & Up & Dmx like 2 \\
\hline ILMN_1745593 & STMN1 & 0.870251 & 0.000142 & 0.002559 & 4.63579 & Up & stathmin 1 \\
\hline ILMN_2330845 & NSF & 1.685669 & 0.000144 & 0.002584 & 4.628835 & Up & N-ethylmaleimide sensitive factor, vesicle fusing ATPase \\
\hline ILMN_1658499 & SYT13 & 1.546667 & 0.000154 & 0.002686 & 4.60051 & Up & synaptotagmin 13 \\
\hline ILMN_2373566 & PJA1 & 0.94189 & 0.000155 & 0.002692 & 4.598467 & Up & praja ring finger ubiquitin ligase 1 \\
\hline ILMN_2342174 & WASF1 & 1.000147 & 0.00016 & 0.002746 & 4.58483 & Up & WAS protein family member 1 \\
\hline ILMN_1787750 & CD200 & 0.969315 & 0.000163 & 0.002784 & 4.577323 & Up & $\begin{array}{l}\text { CD200 molecule } \\
\text { tyrosine 3-monooxygenase/tryptophan 5-monooxygenase }\end{array}$ \\
\hline ILMN_1801928 & YWHAZ & 1.041207 & 0.000165 & 0.0028 & 4.572621 & Up & activation protein zeta \\
\hline ILMN_1763491 & СКМТ1B & 0.920718 & 0.000166 & 0.002807 & 4.569837 & Up & creatine kinase, mitochondrial 1B \\
\hline ILMN_1669631 & GLRB & 1.748696 & 0.000166 & 0.002807 & 4.569747 & Up & glycine receptor beta \\
\hline ILMN_2351638 & BEX4 & 1.116916 & 0.00017 & 0.002841 & 4.55986 & Up & brain expressed X-linked 4 \\
\hline ILMN_2049274 & ARHGDIG & 1.097655 & 0.00017 & 0.002841 & 4.559562 & Up & Rho GDP dissociation inhibitor gamma \\
\hline ILMN_1781987 & CDK5 & 0.900754 & 0.000171 & 0.002854 & 4.556496 & Up & cyclin dependent kinase 5 \\
\hline ILMN_1651826 & BASP1 & 1.227162 & 0.000174 & 0.002881 & 4.549455 & Up & brain abundant membrane attached signal protein 1 \\
\hline ILMN_2080080 & MAP7D2 & 1.664082 & 0.000175 & 0.002887 & 4.547144 & Up & MAP7 domain containing 2 \\
\hline ILMN_1697512 & SLC32A1 & 1.428158 & 0.000176 & 0.002895 & 4.544283 & Up & solute carrier family 32 member 1 \\
\hline ILMN_1661940 & CAMTA1 & 1.1418 & 0.000177 & 0.002901 & 4.542908 & Up & calmodulin binding transcription activator 1 \\
\hline ILMN_1733669 & CDH18 & 1.227135 & 0.000186 & 0.002988 & 4.521046 & Up & cadherin 18 \\
\hline ILMN_1813175 & ADGRL1 & 1.07624 & 0.00019 & 0.00303 & 4.511742 & Up & adhesion G protein-coupled receptor L1 \\
\hline ILMN_1742881 & SYT1 & 2.010766 & 0.000192 & 0.003051 & 4.507944 & Up & synaptotagmin 1 \\
\hline ILMN_2179029 & LRRTM1 & 0.918806 & 0.000194 & 0.003065 & 4.504932 & Up & leucine rich repeat transmembrane neuronal 1 \\
\hline ILMN_1797277 & KIF3C & 1.085299 & 0.000197 & 0.003093 & 4.497691 & Up & kinesin family member $3 \mathrm{C}$ \\
\hline ILMN_1759097 & MLLT11 & 1.68688 & 0.0002 & 0.003117 & 4.491988 & Up & MLLT11, transcription factor 7 cofactor \\
\hline ILMN_1773307 & NAP1L5 & 1.289208 & 0.0002 & 0.003123 & 4.490932 & Up & nucleosome assembly protein 1 like 5 \\
\hline ILMN_1781060 & SYN2 & 1.331032 & 0.000201 & 0.003129 & 4.489421 & Up & synapsin II \\
\hline ILMN_1745130 & RBFOX2 & 0.884037 & 0.000202 & 0.003137 & 4.48675 & Up & RNA binding fox-1 homolog 2 \\
\hline ILMN_1775448 & PFN2 & 1.089696 & 0.000203 & 0.003149 & 4.484038 & Up & profilin 2 \\
\hline ILMN_2060086 & ADAM23 & 0.906705 & 0.000209 & 0.003196 & 4.47374 & Up & ADAM metallopeptidase domain 23 \\
\hline ILMN_1810604 & ELMOD1 & 1.760984 & 0.000209 & 0.003198 & 4.473179 & Up & ELMO domain containing 1 \\
\hline ILMN_1679051 & PTPRR & 0.902585 & 0.000212 & 0.003228 & 4.467051 & Up & protein tyrosine phosphatase, receptor type $\mathrm{R}$ \\
\hline ILMN_1680353 & NSF & 1.448469 & 0.000215 & 0.003244 & 4.461914 & Up & N-ethylmaleimide sensitive factor, vesicle fusing ATPase \\
\hline ILMN_1679580 & KCNIP4 & 1.166354 & 0.00022 & 0.003296 & 4.452236 & Up & potassium voltage-gated channel interacting protein 4 \\
\hline
\end{tabular}


medRxiv preprint doi: https://doi.org/10.1101/2020.12.21.20248688; this version posted December 24, 2020. The copyright holder for this preprint (which was not certified by peer review) is the author/funder, who has granted medRxiv a license to display the preprint in perpetuity. All rights reserved. No reuse allowed without permission.

\begin{tabular}{|c|c|c|c|c|c|c|c|}
\hline ILMN_1804339 & CAMK1G & 1.065073 & 0.000221 & 0.003304 & 4.448736 & Up & calcium/calmodulin dependent protein kinase IG \\
\hline ILMN_1742167 & TUBA1B & 1.299942 & 0.000221 & 0.003304 & 4.448626 & Up & tubulin alpha $1 \mathrm{~b}$ \\
\hline ILMN_1656145 & GOT1 & 1.181531 & 0.000222 & 0.003304 & 4.448432 & Up & glutamic-oxaloacetic transaminase 1 \\
\hline ILMN_1733956 & IARS & 0.931889 & 0.000229 & 0.003365 & 4.433939 & Up & isoleucyl-tRNAsynthetase \\
\hline ILMN_2306189 & MAGED1 & 0.941965 & 0.000231 & 0.003365 & 4.431465 & Up & MAGE family member D1 \\
\hline ILMN_2364700 & ENSA & 1.014412 & 0.000232 & 0.003371 & 4.428839 & Up & endosulfine alpha \\
\hline ILMN_1777261 & FAM3C & 1.265698 & 0.000235 & 0.003404 & 4.423196 & Up & family with sequence similarity 3 member $\mathrm{C}$ \\
\hline ILMN_2397842 & $\mathrm{SNCB}$ & 1.000319 & 0.000236 & 0.003415 & 4.421383 & Up & synuclein beta \\
\hline ILMN_2155816 & DIRAS2 & 1.221898 & 0.00025 & 0.003522 & 4.398712 & Up & DIRAS family GTPase 2 \\
\hline ILMN_1756755 & LINGO1 & 1.137146 & 0.000259 & 0.00359 & 4.382612 & Up & leucine rich repeat and Ig domain containing 1 \\
\hline ILMN_1677829 & SLC9A6 & 0.956883 & 0.000262 & 0.003605 & 4.378914 & Up & solute carrier family 9 member A6 \\
\hline ILMN_1770850 & PNMA1 & 0.950351 & 0.000267 & 0.00365 & 4.370158 & Up & PNMA family member 1 \\
\hline ILMN_1742968 & NMNAT2 & 0.878846 & 0.000271 & 0.003684 & 4.363652 & Up & nicotinamide nucleotide adenylyltransferase 2 \\
\hline ILMN_2223805 & CEP41 & 1.158787 & 0.000273 & 0.003698 & 4.361357 & Up & centrosomal protein 41 \\
\hline ILMN_1758067 & RGS4 & 2.188549 & 0.000278 & 0.003733 & 4.353745 & Up & regulator of $\mathrm{G}$ protein signaling 4 \\
\hline ILMN_1740231 & ELMO1 & 0.904089 & 0.000278 & 0.003733 & 4.353502 & $\mathrm{Up}$ & engulfment and cell motility 1 \\
\hline ILMN_1668411 & FHL2 & 1.200832 & 0.000279 & 0.003749 & 4.351287 & Up & four and a half LIM domains 2 \\
\hline ILMN_1683998 & CREG2 & 1.841295 & 0.000318 & 0.004052 & 4.297566 & $\mathrm{Up}$ & cellular repressor of E1A stimulated genes 2 \\
\hline ILMN_2403730 & ATP6V1H & 0.940966 & 0.000322 & 0.004083 & 4.292045 & Up & ATPase $\mathrm{H}+$ transporting $\mathrm{V} 1$ subunit $\mathrm{H}$ \\
\hline ILMN_2407824 & ATP1B1 & 1.481632 & 0.000328 & 0.004123 & 4.284957 & Up & ATPase $\mathrm{Na}+/ \mathrm{K}+$ transporting subunit beta 1 \\
\hline ILMN_2368773 & FAM3C & 1.226697 & 0.000329 & 0.004129 & 4.283343 & Up & family with sequence similarity 3 member $\mathrm{C}$ \\
\hline ILMN_1685834 & AMPH & 1.531304 & 0.000338 & 0.004185 & 4.271972 & Up & amphiphysin \\
\hline ILMN_1741133 & NME1 & 0.906309 & 0.000339 & 0.004185 & 4.271187 & Up & NME/NM23 nucleoside diphosphate kinase 1 \\
\hline ILMN_1690179 & CRYM & 0.995305 & 0.000339 & 0.004185 & 4.271119 & Up & crystallin mu \\
\hline ILMN_1810376 & C11 orf87 & 1.13745 & 0.000339 & 0.004185 & 4.270706 & Up & chromosome 11 open reading frame 87 \\
\hline ILMN_2205032 & MAGEE1 & 0.894519 & 0.00034 & 0.004189 & 4.269661 & Up & MAGE family member E1 \\
\hline ILMN_1714709 & OLFM1 & 0.961828 & 0.000341 & 0.004194 & 4.268472 & $\mathrm{Up}$ & olfactomedin 1 \\
\hline ILMN_1740500 & TSPYL4 & 1.106939 & 0.000342 & 0.004202 & 4.267048 & Up & TSPY like 4 \\
\hline ILMN_1710209 & MFSD6 & 0.887553 & 0.000343 & 0.004206 & 4.266077 & Up & major facilitator superfamily domain containing 6 \\
\hline ILMN_2171295 & CDK14 & 1.202522 & 0.000343 & 0.004212 & 4.265211 & Up & $\begin{array}{c}\text { cyclin dependent kinase } 14 \\
\text { ATPase sarcoplasmic/endoplasmic reticulum Ca2+ }\end{array}$ \\
\hline ILMN_1655884 & ATP2A2 & 0.879432 & 0.000344 & 0.00422 & 4.264014 & Up & \\
\hline
\end{tabular}




\begin{tabular}{|c|c|c|c|c|c|c|c|}
\hline ILMN_2383516 & WDR7 & 0.866859 & 0.000345 & 0.004222 & 4.263246 & Up & WD repeat domain 7 \\
\hline ILMN_1705153 & NEFH & 1.53841 & 0.000348 & 0.004246 & 4.259455 & Up & neurofilament heavy \\
\hline ILMN_1665856 & CA10 & 0.925833 & 0.00035 & 0.004253 & 4.257756 & Up & carbonic anhydrase 10 \\
\hline ILMN_1687090 & GABRB3 & 1.19619 & 0.000365 & 0.00437 & 4.239761 & Up & gamma-aminobutyric acid type A receptor beta3 subunit \\
\hline ILMN_1699768 & CBLN4 & 1.236672 & 0.00037 & 0.004407 & 4.233629 & Up & $\begin{array}{l}\text { cerebellin } 4 \text { precursor } \\
\text { family with sequence similarity } 19 \text { member A1, C-C motif }\end{array}$ \\
\hline ILMN_1702199 & FAM19A1 & 0.942571 & 0.000379 & 0.004478 & 4.224517 & Up & chemokine like \\
\hline ILMN_1729775 & OPA1 & 0.856929 & 0.000382 & 0.004509 & 4.220622 & Up & OPA1, mitochondrial dynamin like GTPase \\
\hline ILMN_1725417 & NELL2 & 2.014706 & 0.000384 & 0.004517 & 4.219036 & Up & neural EGFL like 2 \\
\hline ILMN_1684461 & CADPS2 & 0.973921 & 0.000389 & 0.00455 & 4.213363 & Up & calcium dependent secretion activator 2 \\
\hline ILMN_1761721 & VPS35 & 0.874961 & 0.000391 & 0.004566 & 4.210599 & Up & VPS35, retromer complex component \\
\hline ILMN_1730291 & ATP1B1 & 1.340271 & 0.000395 & 0.00459 & 4.207189 & Up & ATPase $\mathrm{Na}+\mathrm{K}+$ transporting subunit beta 1 \\
\hline ILMN_1805665 & FLRT3 & 1.078651 & 0.000395 & 0.004594 & 4.206621 & Up & fibronectinleucine rich transmembrane protein 3 \\
\hline ILMN_1697561 & FBXL16 & 1.16672 & 0.000397 & 0.004606 & 4.204781 & Up & F-box and leucine rich repeat protein 16 \\
\hline ILMN_1663397 & CAMK2B & 1.029691 & 0.000398 & 0.004613 & 4.203299 & Up & calcium/calmodulin dependent protein kinase II beta \\
\hline ILMN_1682428 & HENMT1 & 0.885948 & 0.000401 & 0.004622 & 4.200864 & Up & HEN methyltransferase 1 \\
\hline ILMN_1736441 & PDXP & 1.001109 & 0.000401 & 0.004622 & 4.200707 & Up & pyridoxal phosphatase \\
\hline ILMN_1690223 & CNTNAP2 & 1.225355 & 0.000401 & 0.004622 & 4.200567 & Up & contactin associated protein like 2 \\
\hline ILMN_1754570 & KCTD8 & 0.997214 & 0.000403 & 0.004636 & 4.198879 & Up & potassium channel tetramerization domain containing 8 \\
\hline ILMN_1704383 & TRIM37 & 1.475103 & 0.000406 & 0.004652 & 4.195738 & Up & tripartite motif containing 37 \\
\hline ILMN_1784207 & PITHD1 & 0.958834 & 0.000409 & 0.00467 & 4.192505 & Up & PITH domain containing 1 \\
\hline ILMN_1660718 & GABBR2 & 1.784204 & 0.000411 & 0.004688 & 4.190524 & Up & gamma-aminobutyric acid type B receptor subunit 2 \\
\hline ILMN_2129910 & SLC12A5 & 1.570048 & 0.000413 & 0.004716 & 4.187814 & Up & solute carrier family 12 member 5 \\
\hline ILMN_2359601 & CAMK2G & 0.869404 & 0.000416 & 0.00473 & 4.185576 & $\mathrm{Up}$ & calcium/calmodulin dependent protein kinase II gamma \\
\hline ILMN_2198594 & RFPL1S & 0.919892 & 0.000416 & 0.004732 & 4.184871 & Up & RFPL1 antisense RNA 1 \\
\hline ILMN_2388484 & MAP2 & 1.008756 & 0.000425 & 0.004792 & 4.176168 & Up & $\begin{array}{c}\text { microtubule associated protein } 2 \\
\text { tyrosine 3-monooxygenase/tryptophan 5-monooxygenase }\end{array}$ \\
\hline ILMN_1728512 & YWHAH & 0.990966 & 0.000427 & 0.004804 & 4.174055 & $\mathrm{Up}$ & $\begin{array}{l}\text { activation protein eta } \\
\text { gamma-aminobutyric acid type A receptor gamma2 }\end{array}$ \\
\hline ILMN_2413964 & GABRG2 & 1.817738 & 0.000428 & 0.004804 & 4.173736 & Up & subunit \\
\hline ILMN_2096623 & SLITRK1 & 1.137666 & 0.000428 & 0.004805 & 4.173471 & Up & SLIT and NTRK like family member 1 \\
\hline ILMN_1669382 & CPLX2 & 1.140671 & 0.000434 & 0.004846 & 4.167067 & $\mathrm{Up}$ & complexin 2 \\
\hline ILMN_2354547 & TUSC3 & 1.033281 & 0.000441 & 0.004907 & 4.160685 & Up & tumor suppressor candidate 3 \\
\hline ILMN_1764201 & MAP2 & 1.015209 & 0.000447 & 0.004938 & 4.155227 & Up & microtubule associated protein 2 \\
\hline ILMN_1672229 & FGF12 & 0.998556 & 0.00047 & 0.005087 & 4.134378 & $\mathrm{Up}$ & fibroblast growth factor 12 \\
\hline ILMN_1771622 & CALY & 1.054178 & 0.00047 & 0.005088 & 4.134121 & $\mathrm{Up}$ & calcyon neuron specific vesicular protein \\
\hline ILMN_1787705 & ATP6V1B2 & 0.951592 & 0.000471 & 0.00509 & 4.133231 & Up & ATPase $\mathrm{H}+$ transporting $\mathrm{V} 1$ subunit $\mathrm{B} 2$ \\
\hline ILMN_1788250 & LDOC1 & 0.887884 & 0.000474 & 0.00511 & 4.1306 & Up & LDOC1, regulator of NFKB signaling \\
\hline ILMN_2069224 & PVALB & 2.097766 & 0.000475 & 0.00511 & 4.130032 & Up & parvalbumin \\
\hline ILMN_1757384 & RAN & 0.936454 & 0.00048 & 0.005148 & 4.125198 & Up & RAN, member RAS oncogene family \\
\hline ILMN_1798254 & ACTR10 & 0.861182 & 0.000485 & 0.005175 & 4.121377 & Up & actin related protein 10 homolog \\
\hline ILMN_1806306 & SV2B & 1.422749 & 0.00049 & 0.005196 & 4.11658 & Up & synaptic vesicle glycoprotein 2B \\
\hline ILMN_1716265 & PGM2L1 & 1.695377 & 0.000493 & 0.005222 & 4.114195 & Up & phosphoglucomutase 2 like 1 \\
\hline ILMN_1678493 & CHN1 & 1.375119 & 0.000501 & 0.005259 & 4.107589 & $\mathrm{Up}$ & $\begin{array}{l}\text { chimerin } 1 \\
\text { arcoplasmic/endoplasmic reticulum }\end{array}$ \\
\hline ILMN_1687375 & ATP2A2 & 1.021708 & 0.000512 & 0.005324 & 4.098487 & $\mathrm{Up}$ & transporting 2 \\
\hline
\end{tabular}




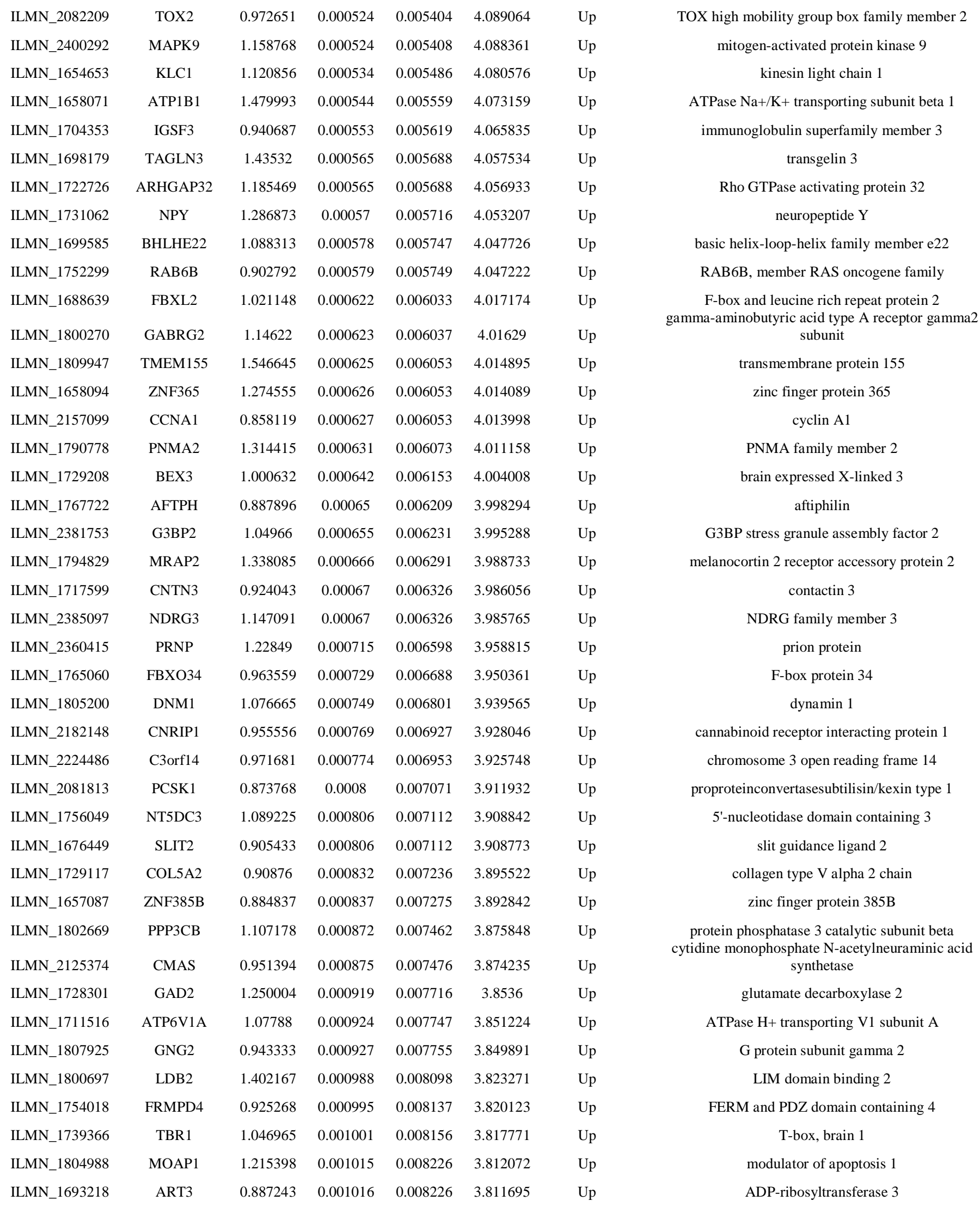




\begin{tabular}{|c|c|c|c|c|c|c|c|}
\hline ILMN_2103547 & GOLGA8B & 0.973208 & 0.001042 & 0.00836 & 3.801114 & Up & golgin A8 family member B \\
\hline ILMN_2412822 & $\mathrm{SCN} 3 \mathrm{~B}$ & 1.376592 & 0.001054 & 0.008429 & 3.796206 & Up & sodium voltage-gated channel beta subunit 3 \\
\hline ILMN_1766165 & SNCA & 1.323592 & 0.001058 & 0.008452 & 3.794361 & Up & synuclein alpha \\
\hline ILMN_1800059 & KCNIP4 & 0.937112 & 0.001076 & 0.008548 & 3.787346 & Up & potassium voltage-gated channel interacting protein 4 \\
\hline ILMN_1788538 & NCALD & 1.308467 & 0.0011 & 0.008678 & 3.778363 & Up & neurocalcin delta \\
\hline ILMN_1698885 & PTPRT & 1.187645 & 0.001119 & 0.008781 & 3.771022 & Up & protein tyrosine phosphatase, receptor type $\mathrm{T}$ \\
\hline ILMN_2292646 & GAD1 & 1.504949 & 0.001124 & 0.008811 & 3.76921 & Up & glutamate decarboxylase 1 \\
\hline ILMN_1783805 & PNMA3 & 1.057284 & 0.001139 & 0.008876 & 3.763584 & Up & PNMA family member 3 \\
\hline ILMN_1668125 & MYRIP & 0.881157 & 0.001151 & 0.008941 & 3.759166 & Up & myosin VIIA and Rab interacting protein \\
\hline ILMN_2068747 & OAT & 0.93165 & 0.001159 & 0.008988 & 3.75641 & Up & ornithine aminotransferase \\
\hline ILMN_1709809 & SNU13 & 1.062798 & 0.001208 & 0.009244 & 3.738696 & Up & small nuclear ribonucleoprotein 13 \\
\hline ILMN_1715384 & B4GAT1 & 1.216566 & 0.001274 & 0.00959 & 3.716501 & Up & beta-1,4-glucuronyltransferase 1 \\
\hline ILMN_2333367 & FKBP1A & 1.053811 & 0.00129 & 0.009661 & 3.711169 & Up & FK506 binding protein $1 \mathrm{~A}$ \\
\hline ILMN_2316173 & AP1S1 & 1.083351 & 0.001291 & 0.009661 & 3.711049 & Up & adaptor related protein complex 1 subunit sigma 1 \\
\hline ILMN_1767503 & GABRB3 & 0.997851 & 0.001301 & 0.009719 & 3.707638 & Up & gamma-aminobutyric acid type A receptor beta3 subunit \\
\hline ILMN_1797950 & EXTL2 & 0.912675 & 0.001305 & 0.009736 & 3.706276 & Up & $\begin{array}{l}\text { exostosin like glycosyltransferase } 2 \\
\text { protein kinase cAMP-dependent type I regulatory subunit }\end{array}$ \\
\hline ILMN_1674390 & PRKAR1B & 1.265797 & 0.001322 & 0.009824 & 3.700847 & Up & beta \\
\hline ILMN_1791593 & DENND5B & 0.878818 & 0.001337 & 0.009891 & 3.6963 & Up & DENN domain containing 5B \\
\hline ILMN_1728803 & NSG2 & 0.988385 & 0.001392 & 0.010185 & 3.679377 & Up & neuronal vesicle trafficking associated 2 \\
\hline ILMN_1757081 & SYN2 & 1.519785 & 0.001413 & 0.010306 & 3.672987 & Up & synapsin II \\
\hline ILMN_1794490 & PNMA8A & 0.870046 & 0.001424 & 0.010365 & 3.669552 & Up & PNMA family member $8 \mathrm{~A}$ \\
\hline ILMN_1661491 & SH3GL2 & 1.214223 & 0.001473 & 0.010608 & 3.65528 & Up & SH3 domain containing GRB2 like 2, endophilin A1 \\
\hline ILMN_1671149 & MEG3 & 0.991892 & 0.001486 & 0.010678 & 3.651581 & Up & maternally expressed 3 \\
\hline ILMN_1684771 & PGRMC1 & 0.869092 & 0.001492 & 0.010702 & 3.650146 & Up & progesterone receptor membrane component 1 \\
\hline ILMN_1716988 & OPN3 & 1.017428 & 0.001503 & 0.010749 & 3.646806 & Up & opsin 3 \\
\hline ILMN_1756715 & RUNDC3A & 1.0861 & 0.001555 & 0.01096 & 3.63251 & Up & RUN domain containing $3 \mathrm{~A}$ \\
\hline ILMN_1711327 & TRIM37 & 0.866207 & 0.00157 & 0.011041 & 3.62844 & $\mathrm{Up}$ & tripartite motif containing 37 \\
\hline ILMN_2065022 & ARHGAP44 & 0.880778 & 0.001725 & 0.011814 & 3.588849 & Up & Rho GTPase activating protein 44 \\
\hline ILMN_1687768 & NCOA7 & 0.901733 & 0.001733 & 0.011851 & 3.586802 & Up & nuclear receptor coactivator 7 \\
\hline ILMN_1748983 & RTN4 & 1.414516 & 0.001738 & 0.011874 & 3.585694 & Up & $\begin{array}{c}\text { reticulon } 4 \\
\text { family with sequence similarity } 19 \text { member A2, C-C motif }\end{array}$ \\
\hline ILMN_2172318 & FAM19A2 & 0.958634 & 0.001846 & 0.012418 & 3.560162 & Up & \\
\hline ILMN_1698323 & PLEKHB2 & 1.009521 & 0.001865 & 0.012493 & 3.555748 & Up & pleckstrin homology domain containing B2 \\
\hline ILMN_1802082 & PRDM8 & 0.876177 & 0.001886 & 0.012588 & 3.551142 & $\mathrm{Up}$ & PR/SET domain 8 \\
\hline ILMN_2384409 & TAC1 & 0.85989 & 0.00194 & 0.012787 & 3.539145 & Up & tachykinin precursor 1 \\
\hline ILMN_1762899 & EGR1 & 0.92929 & 0.00209 & 0.013475 & 3.507603 & Up & early growth response 1 \\
\hline ILMN_1701933 & SNCA & 1.414152 & 0.002248 & 0.014163 & 3.476811 & Up & synuclein alpha \\
\hline ILMN_1698846 & SLC8A2 & 1.119861 & 0.002336 & 0.014532 & 3.460477 & Up & solute carrier family 8 member A2 \\
\hline ILMN_2148469 & RASL11B & 1.260365 & 0.002343 & 0.014547 & 3.459144 & $\mathrm{Up}$ & RAS like family 11 member B \\
\hline ILMN_1672094 & DLX1 & 0.898442 & 0.002363 & 0.014636 & 3.45553 & Up & distal-less homeobox 1 \\
\hline ILMN_2112417 & PGAM1 & 0.892946 & 0.002462 & 0.015025 & 3.43812 & Up & phosphoglyceratemutase 1 \\
\hline ILMN_1812824 & SST & 1.543958 & 0.002582 & 0.01553 & 3.417852 & $\mathrm{Up}$ & somatostatin \\
\hline ILMN_1694240 & MAP2K1 & 1.007904 & 0.002585 & 0.015536 & 3.417495 & $\mathrm{Up}$ & mitogen-activated protein kinase kinase 1 \\
\hline
\end{tabular}




\begin{tabular}{|c|c|c|c|c|c|c|c|}
\hline ILMN_1737462 & OXR1 & 0.925948 & 0.002631 & 0.015708 & 3.409883 & Up & oxidation resistance 1 \\
\hline ILMN_1713561 & LAMP5 & 1.16465 & 0.002686 & 0.015932 & 3.401186 & Up & lysosomal associated membrane protein family member 5 \\
\hline ILMN_1785191 & TMEM14A & 1.043406 & 0.002696 & 0.015972 & 3.399558 & Up & transmembrane protein $14 \mathrm{~A}$ \\
\hline ILMN_1755428 & CABP1 & 0.860897 & 0.002941 & 0.017008 & 3.362521 & Up & calcium binding protein 1 \\
\hline ILMN_2188722 & GLS & 1.038939 & 0.003007 & 0.01733 & 3.35295 & Up & glutaminase \\
\hline ILMN_1736700 & ALDOA & 1.070599 & 0.003373 & 0.018834 & 3.303849 & Up & aldolase, fructose-bisphosphate A \\
\hline ILMN_1769195 & ERC2 & 0.886673 & 0.003646 & 0.019933 & 3.27057 & Up & ELKS/RAB6-interacting/CAST family member 2 \\
\hline ILMN_2320906 & RTN3 & 0.861172 & 0.003678 & 0.020036 & 3.266831 & Up & reticulon 3 \\
\hline ILMN_2065773 & SCG5 & 0.877681 & 0.003838 & 0.02062 & 3.248563 & Up & secretogranin $\mathrm{V}$ \\
\hline ILMN_1691237 & CAP2 & 1.107805 & 0.003973 & 0.021136 & 3.233725 & Up & cyclase associated actin cytoskeleton regulatory protein 2 \\
\hline ILMN_1801302 & SCN1B & 1.133305 & 0.004066 & 0.021487 & 3.223746 & Up & sodium voltage-gated channel beta subunit 1 \\
\hline ILMN_2322552 & NCKAP1 & 0.898556 & 0.004172 & 0.021856 & 3.212655 & Up & NCK associated protein 1 \\
\hline ILMN_1656537 & SNRPN & 1.026159 & 0.004316 & 0.022429 & 3.198027 & Up & small nuclear ribonucleoprotein polypeptide $\mathrm{N}$ \\
\hline ILMN_2172497 & PLPPR4 & 0.911827 & 0.00438 & 0.022663 & 3.191732 & Up & phospholipid phosphatase related 4 \\
\hline ILMN_1814770 & CPNE4 & 1.017515 & 0.004448 & 0.022948 & 3.185067 & Up & copine 4 \\
\hline ILMN_1669410 & CHGA & 1.166899 & 0.004682 & 0.023808 & 3.16298 & Up & chromogranin A \\
\hline ILMN_1800179 & $\mathrm{KCNJ} 4$ & 1.043876 & 0.0047 & 0.023852 & 3.161273 & Up & potassium voltage-gated channel subfamily J member 4 \\
\hline ILMN_2151114 & VSNL1 & 0.884021 & 0.004768 & 0.024087 & 3.155094 & Up & visinin like 1 \\
\hline ILMN_1779147 & ENC1 & 1.764661 & 0.004801 & 0.024192 & 3.15214 & Up & ectodermal-neural cortex 1 \\
\hline ILMN_1659086 & NEFL & 1.036968 & 0.004808 & 0.024209 & 3.151492 & Up & neurofilament light \\
\hline ILMN_1691413 & NNAT & 1.004985 & 0.004822 & 0.024267 & 3.15019 & Up & neuronatin \\
\hline ILMN_1705686 & NRGN & 1.410225 & 0.005315 & 0.026071 & 3.108125 & Up & neurogranin \\
\hline ILMN_2218758 & NECAB1 & 1.077346 & 0.005426 & 0.026446 & 3.099143 & Up & N-terminal EF-hand calcium binding protein 1 \\
\hline ILMN_2387712 & AK5 & 1.241329 & 0.0058 & 0.027719 & 3.070203 & Up & adenylate kinase 5 \\
\hline ILMN_1815033 & HPCA & 0.883788 & 0.006283 & 0.029465 & 3.035337 & Up & hippocalcin \\
\hline ILMN_1805807 & SLC30A3 & 0.945231 & 0.006471 & 0.030088 & 3.022478 & Up & solute carrier family 30 member 3 \\
\hline ILMN_1757497 & VGF & 1.293038 & 0.006572 & 0.030442 & 3.015722 & Up & VGF nerve growth factor inducible \\
\hline ILMN_1703178 & SCG2 & 1.073561 & 0.006878 & 0.031403 & 2.995818 & Up & secretogranin II \\
\hline ILMN_1712943 & $\mathrm{CCK}$ & 1.284335 & 0.0072 & 0.032517 & 2.975772 & Up & cholecystokinin \\
\hline ILMN_1776121 & KIAA1211L & 1.011563 & 0.007417 & 0.033207 & 2.962798 & Up & KIAA1211 like \\
\hline ILMN_2404063 & APP & 1.103321 & 0.007971 & 0.035055 & 2.931134 & Up & amyloid beta precursor protein \\
\hline ILMN_1666904 & SLC17A6 & 0.933499 & 0.010996 & 0.044237 & 2.788597 & Up & solute carrier family 17 member 6 \\
\hline ILMN_2404065 & APP & 1.04849 & 0.011234 & 0.04489 & 2.779017 & Up & amyloid beta precursor protein \\
\hline ILMN_1768758 & CACNG3 & 1.100505 & 0.012521 & 0.048569 & 2.73043 & Up & calcium voltage-gated channel auxiliary subunit gamma 3 \\
\hline ILMN_1671489 & $\mathrm{PC}$ & -0.87051 & $1.49 \mathrm{E}-08$ & 0.000122 & -8.87107 & Down & pyruvate carboxylase \\
\hline ILMN_1774836 & PLOD3 & -0.9444 & 4.67E-08 & 0.000191 & -8.2785 & Down & procollagen-lysine,2-oxoglutarate 5-dioxygenase 3 \\
\hline ILMN_1781400 & SLC7A2 & -2.01982 & $8.29 \mathrm{E}-08$ & 0.000192 & -7.99016 & Down & solute carrier family 7 member 2 \\
\hline ILMN_1729216 & CRYAB & -1.18385 & 2.03E-07 & 0.000266 & -7.55019 & Down & crystallin alpha B \\
\hline ILMN_1684391 & PLOD1 & -1.10563 & $2.28 \mathrm{E}-07$ & 0.000266 & -7.49528 & Down & procollagen-lysine,2-oxoglutarate 5-dioxygenase 1 \\
\hline ILMN_1793770 & DNAJB6 & -0.92558 & $3.17 \mathrm{E}-07$ & 0.000305 & -7.33637 & Down & DnaJ heat shock protein family (Hsp40) member B6 \\
\hline ILMN_1758315 & SLC9A9 & -0.86852 & $3.23 \mathrm{E}-07$ & 0.000305 & -7.327 & Down & solute carrier family 9 member A9 \\
\hline ILMN_1702487 & SGK1 & -0.95517 & 4.11E-07 & 0.000337 & -7.21293 & Down & serum/glucocorticoid regulated kinase 1 \\
\hline ILMN_2071937 & ATP6V0E1 & -1.21139 & $4.18 \mathrm{E}-07$ & 0.000337 & -7.20474 & Down & ATPase $\mathrm{H}+$ transporting V0 subunit e1 \\
\hline
\end{tabular}




\begin{tabular}{|c|c|c|c|c|c|c|c|}
\hline ILMN_2149494 & NPL & -1.23256 & $4.39 \mathrm{E}-07$ & 0.000337 & -7.18162 & Down & $\mathrm{N}$-acetylneuraminate pyruvate lyase \\
\hline ILMN_1769091 & PRCP & -0.9903 & $5.47 \mathrm{E}-07$ & 0.000354 & -7.07861 & Down & prolylcarboxypeptidase \\
\hline ILMN_1810559 & RHOQ & -1.31603 & 7.04E-07 & 0.000381 & -6.96075 & Down & ras homolog family member $\mathrm{Q}$ \\
\hline ILMN_1681679 & TSPO & -1.65071 & $7.62 \mathrm{E}-07$ & 0.000381 & -6.92385 & Down & translocator protein \\
\hline ILMN_2305544 & DBI & -0.88496 & $9.59 \mathrm{E}-07$ & 0.000408 & -6.81698 & Down & diazepam binding inhibitor, acyl-CoA binding protein \\
\hline ILMN_1801441 & RFTN2 & -1.14454 & $9.86 \mathrm{E}-07$ & 0.000408 & -6.804 & Down & raftlin family member 2 \\
\hline ILMN_2064725 & METTL7B & -1.57323 & $1.08 \mathrm{E}-06$ & 0.000422 & -6.76357 & Down & methyltransferase like 7B \\
\hline ILMN_1783231 & PLEKHB1 & -1.22052 & $1.11 \mathrm{E}-06$ & 0.000427 & -6.74766 & Down & pleckstrin homology domain containing B1 \\
\hline ILMN_1655612 & ARRDC2 & -0.92216 & $1.2 \mathrm{E}-06$ & 0.000453 & -6.71366 & Down & arrestin domain containing 2 \\
\hline ILMN_2352303 & RASSF2 & -1.05568 & $1.63 \mathrm{E}-06$ & 0.000503 & -6.57449 & Down & Ras association domain family member 2 \\
\hline ILMN_1779448 & EFHD1 & -1.00984 & $1.68 \mathrm{E}-06$ & 0.000503 & -6.56108 & Down & EF-hand domain family member D1 \\
\hline ILMN_1696537 & DDIT4L & -1.36563 & $1.68 \mathrm{E}-06$ & 0.000503 & -6.55984 & Down & DNA damage inducible transcript 4 like \\
\hline ILMN_1758731 & CYP2J2 & -1.01095 & $1.74 \mathrm{E}-06$ & 0.000504 & -6.54309 & Down & cytochrome P450 family 2 subfamily J member 2 \\
\hline ILMN_1707503 & SZRD1 & -0.97749 & $1.75 \mathrm{E}-06$ & 0.000504 & -6.54202 & Down & SUZ RNA binding domain containing 1 \\
\hline ILMN_1661599 & DDIT4 & -1.58688 & $1.82 \mathrm{E}-06$ & 0.000517 & -6.52333 & Down & DNA damage inducible transcript 4 \\
\hline ILMN_1778977 & TYROBP & -2.11065 & $1.83 \mathrm{E}-06$ & 0.000517 & -6.52037 & Down & TYRO protein tyrosine kinase binding protein \\
\hline ILMN_1811921 & CSRP1 & -1.08353 & $1.97 \mathrm{E}-06$ & 0.000539 & -6.48767 & Down & cysteine and glycine rich protein 1 \\
\hline ILMN_1803423 & ARHGEF6 & -1.03039 & $2.49 \mathrm{E}-06$ & 0.000574 & -6.38139 & Down & Rac/Cdc42 guanine nucleotide exchange factor 6 \\
\hline ILMN_1749011 & NECAP2 & -0.99525 & $2.51 \mathrm{E}-06$ & 0.000574 & -6.37824 & Down & NECAP endocytosis associated 2 \\
\hline ILMN_2349658 & TSPO & -1.05416 & $2.7 \mathrm{E}-06$ & 0.000584 & -6.34512 & Down & translocator protein \\
\hline ILMN_1660021 & PLIN3 & -1.05105 & 2.77E-06 & 0.000584 & -6.33357 & Down & perilipin 3 \\
\hline ILMN_1767523 & IL17RB & -1.06383 & $2.8 \mathrm{E}-06$ & 0.000584 & -6.32947 & Down & interleukin 17 receptor B \\
\hline ILMN_2390853 & CTSH & -1.25338 & $2.9 \mathrm{E}-06$ & 0.000584 & -6.31411 & Down & cathepsin $\mathrm{H}$ \\
\hline ILMN_1720282 & NQO1 & -1.8993 & $2.9 \mathrm{E}-06$ & 0.000584 & -6.31404 & Down & $\mathrm{NAD}(\mathrm{P}) \mathrm{H}$ quinone dehydrogenase 1 \\
\hline ILMN_1812262 & DDR1 & -1.14337 & $3.18 \mathrm{E}-06$ & 0.000599 & -6.27213 & Down & discoidin domain receptor tyrosine kinase 1 \\
\hline ILMN_1692938 & PSAT1 & -1.09161 & $3.18 \mathrm{E}-06$ & 0.000599 & -6.27147 & Down & phosphoserine aminotransferase 1 \\
\hline ILMN_1695851 & PARVG & -1.71728 & $3.23 \mathrm{E}-06$ & 0.000599 & -6.26522 & Down & parvin gamma \\
\hline ILMN_1698732 & PALLD & -1.27866 & $3.63 \mathrm{E}-06$ & 0.000613 & -6.21341 & Down & palladin, cytoskeletal associated protein \\
\hline ILMN_1713124 & AKR1C3 & -1.22642 & 3.67E-06 & 0.000613 & -6.20794 & Down & aldo-ketoreductase family 1 member $\mathrm{C} 3$ \\
\hline ILMN_1785926 & ZNF621 & -0.90867 & $3.82 \mathrm{E}-06$ & 0.000613 & -6.18986 & Down & zinc finger protein 621 \\
\hline ILMN_2407389 & GPNMB & -0.86527 & $4.18 \mathrm{E}-06$ & 0.000613 & -6.14984 & Down & glycoprotein nmb \\
\hline ILMN_1719599 & SYTL4 & -1.47637 & 4.23E-06 & 0.000613 & -6.14469 & Down & synaptotagmin like 4 \\
\hline
\end{tabular}




\begin{tabular}{|c|c|c|c|c|c|c|c|}
\hline ILMN_1673232 & CERS1 & -0.90409 & $4.28 \mathrm{E}-06$ & 0.000613 & -6.13915 & Down & ceramide synthase 1 \\
\hline ILMN_1787813 & SLC5A3 & -1.24295 & $4.42 \mathrm{E}-06$ & 0.000613 & -6.12566 & Down & solute carrier family 5 member 3 \\
\hline ILMN_1722981 & TLR5 & -1.24313 & $4.42 \mathrm{E}-06$ & 0.000613 & -6.12552 & Down & toll like receptor 5 \\
\hline ILMN_1651315 & HMG20B & -1.0812 & $4.45 \mathrm{E}-06$ & 0.000613 & -6.12236 & Down & high mobility group $20 \mathrm{~B}$ \\
\hline ILMN_1778444 & FKBP5 & -1.09012 & $4.52 \mathrm{E}-06$ & 0.000619 & -6.11539 & Down & FK506 binding protein 5 \\
\hline ILMN_1669409 & VSIG4 & -1.56578 & $4.58 \mathrm{E}-06$ & 0.000621 & -6.10966 & Down & V-set and immunoglobulin domain containing 4 \\
\hline ILMN_1772359 & LAPTM5 & -1.80203 & $4.89 \mathrm{E}-06$ & 0.000647 & -6.08027 & Down & lysosomal protein transmembrane 5 \\
\hline ILMN_1701877 & AXL & -1.42481 & $4.9 \mathrm{E}-06$ & 0.000647 & -6.07932 & Down & AXL receptor tyrosine kinase \\
\hline ILMN_2094856 & RANBP3L & -1.49822 & 4.96E-06 & 0.000647 & -6.07461 & Down & RAN binding protein 3 like \\
\hline ILMN_1682139 & RAI14 & -0.94545 & $5.16 \mathrm{E}-06$ & 0.000657 & -6.05648 & Down & retinoic acid induced 14 \\
\hline ILMN_1752478 & DHRS3 & -0.90409 & $5.19 \mathrm{E}-06$ & 0.000657 & -6.05419 & Down & dehydrogenase/reductase 3 \\
\hline ILMN_1705442 & CMTM3 & -1.19361 & $5.21 \mathrm{E}-06$ & 0.000657 & -6.05201 & Down & CKLF like MARVEL transmembrane domain containing 3 \\
\hline ILMN_1795865 & FGFRL1 & -1.77628 & $5.55 \mathrm{E}-06$ & 0.000667 & -6.0245 & Down & fibroblast growth factor receptor like 1 \\
\hline ILMN_1754660 & $\mathrm{ZCCHC} 24$ & -1.10333 & $5.55 \mathrm{E}-06$ & 0.000667 & -6.02429 & Down & zinc finger CCHC-type containing 24 \\
\hline ILMN_1727458 & HDAC1 & -0.94915 & $5.6 \mathrm{E}-06$ & 0.000667 & -6.02055 & Down & histone deacetylase 1 \\
\hline ILMN_2348367 & FGFRL1 & -1.07157 & $5.75 \mathrm{E}-06$ & 0.000668 & -6.00916 & Down & fibroblast growth factor receptor like 1 \\
\hline ILMN_2260991 & TSPO & -0.84244 & $5.87 \mathrm{E}-06$ & 0.000676 & -5.99955 & Down & translocator protein \\
\hline ILMN_1657683 & C1orf198 & -0.94546 & $5.92 \mathrm{E}-06$ & 0.000676 & -5.99591 & Down & chromosome 1 open reading frame 198 \\
\hline ILMN_1799725 & DOCK2 & -1.12419 & $6.15 \mathrm{E}-06$ & 0.000692 & -5.97925 & Down & dedicator of cytokinesis 2 \\
\hline ILMN_1809477 & CARHSP1 & -0.90202 & $6.31 \mathrm{E}-06$ & 0.0007 & -5.96783 & Down & calcium regulated heat stable protein 1 \\
\hline ILMN_1810560 & NUPR1 & -1.12935 & $6.47 \mathrm{E}-06$ & 0.0007 & -5.95699 & Down & nuclear protein 1 , transcriptional regulator \\
\hline ILMN_1732452 & MAPKAPK3 & -1.22959 & $6.47 \mathrm{E}-06$ & 0.0007 & -5.95662 & Down & mitogen-activated protein kinase-activated protein kinase 3 \\
\hline ILMN_1800512 & HMOX1 & -1.04217 & $6.81 \mathrm{E}-06$ & 0.000708 & -5.93414 & Down & hemeoxygenase 1 \\
\hline ILMN_1718769 & ITSN1 & -1.13479 & $6.93 \mathrm{E}-06$ & 0.000715 & -5.92645 & Down & intersectin 1 \\
\hline ILMN_1809522 & NACC2 & -1.10616 & 7.03E-06 & 0.000716 & -5.92038 & Down & NACC family member 2 \\
\hline ILMN_1752075 & MYBPC1 & -1.60214 & 7.49E-06 & 0.000729 & -5.89251 & Down & myosin binding protein $\mathrm{C}$, slow type \\
\hline ILMN_2123743 & FCER1G & -1.95198 & $7.53 \mathrm{E}-06$ & 0.000729 & -5.89041 & Down & $\mathrm{Fc}$ fragment of $\mathrm{IgE}$ receptor Ig \\
\hline ILMN_1714527 & VAMP3 & -0.87899 & $7.65 \mathrm{E}-06$ & 0.000733 & -5.88337 & Down & vesicle associated membrane protein 3 \\
\hline ILMN_1668374 & ITGB5 & -1.20897 & $8.05 \mathrm{E}-06$ & 0.000756 & -5.86065 & Down & integrin subunit beta 5 \\
\hline ILMN_1791280 & HSPB8 & -0.85424 & $8.39 \mathrm{E}-06$ & 0.000765 & -5.84272 & Down & heat shock protein family B (small) member 8 \\
\hline ILMN_1796409 & C1QB & -2.50406 & 8.57E-06 & 0.000776 & -5.83334 & Down & complement C1q B chain \\
\hline ILMN_1716678 & NPC2 & -1.28946 & $8.8 \mathrm{E}-06$ & 0.000781 & -5.82191 & Down & NPC intracellular cholesterol transporter 2 \\
\hline ILMN_2360784 & RRBP1 & -1.04661 & $8.92 \mathrm{E}-06$ & 0.000785 & -5.81591 & Down & ribosome binding protein 1 \\
\hline ILMN_1693826 & HAVCR2 & -2.10807 & $9.21 \mathrm{E}-06$ & 0.000785 & -5.80185 & Down & hepatitis A virus cellular receptor 2 \\
\hline ILMN_1789007 & APOC1 & -2.32 & $9.28 \mathrm{E}-06$ & 0.000785 & -5.7987 & Down & apolipoprotein $\mathrm{C} 1$ \\
\hline ILMN_2190084 & VAMP8 & -1.60976 & $9.38 \mathrm{E}-06$ & 0.000788 & -5.79414 & Down & vesicle associated membrane protein 8 \\
\hline ILMN_1684158 & GPT2 & -1.0484 & $9.69 \mathrm{E}-06$ & 0.000798 & -5.77969 & Down & glutamic--pyruvic transaminase 2 \\
\hline ILMN_1776861 & HAP1 & -1.06409 & $9.83 \mathrm{E}-06$ & 0.000802 & -5.77355 & Down & huntingtin associated protein 1 \\
\hline ILMN_1668345 & $\mathrm{OAF}$ & -1.61698 & $1 \mathrm{E}-05$ & 0.000809 & -5.76587 & Down & out at first homolog \\
\hline ILMN_1660871 & NEK6 & -1.10464 & $1.01 \mathrm{E}-05$ & 0.00081 & -5.76135 & Down & NIMA related kinase 6 \\
\hline ILMN_1662932 & LCP1 & -1.86774 & $1.01 \mathrm{E}-05$ & 0.00081 & -5.76056 & Down & lymphocyte cytosolic protein 1 \\
\hline ILMN_2364521 & AXL & -1.33863 & $1.03 \mathrm{E}-05$ & 0.00081 & -5.7527 & Down & AXL receptor tyrosine kinase \\
\hline ILMN_2044453 & LPAR5 & -1.53377 & $1.05 \mathrm{E}-05$ & 0.00081 & -5.74464 & Down & lysophosphatidic acid receptor 5 \\
\hline
\end{tabular}




\begin{tabular}{|c|c|c|c|c|c|c|c|}
\hline ILMN_1698934 & CMTM7 & -1.27691 & $1.07 \mathrm{E}-05$ & 0.000812 & -5.73688 & Down & CKLF like MARVEL transmembrane domain containing 7 \\
\hline ILMN_1651354 & SPP1 & -1.98642 & $1.08 \mathrm{E}-05$ & 0.000814 & -5.73153 & Down & secreted phosphoprotein 1 \\
\hline ILMN_2282641 & TBXAS1 & -1.29648 & $1.12 \mathrm{E}-05$ & 0.000823 & -5.71518 & Down & thromboxane A synthase 1 \\
\hline ILMN_2216582 & LYL1 & -1.27072 & $1.22 \mathrm{E}-05$ & 0.000864 & -5.68136 & Down & LYL1, basic helix-loop-helix family member \\
\hline ILMN_1796455 & RYR3 & -1.28249 & $1.22 \mathrm{E}-05$ & 0.000864 & -5.67995 & Down & ryanodine receptor 3 \\
\hline ILMN_1693014 & СЕВPB & -1.07582 & $1.27 \mathrm{E}-05$ & 0.000877 & -5.66128 & Down & CCAAT enhancer binding protein beta \\
\hline ILMN_2175912 & ITGB2 & -2.16115 & $1.27 \mathrm{E}-05$ & 0.000877 & -5.66103 & Down & integrin subunit beta 2 \\
\hline ILMN_1709683 & RASSF2 & -1.05164 & $1.27 \mathrm{E}-05$ & 0.000877 & -5.661 & Down & Ras association domain family member 2 \\
\hline ILMN_2059549 & SYK & -1.14395 & $1.29 \mathrm{E}-05$ & 0.000884 & -5.65538 & Down & spleen associated tyrosine kinase \\
\hline ILMN_1730201 & DTNA & -1.81488 & $1.31 \mathrm{E}-05$ & 0.000889 & -5.64772 & Down & dystrobrevin alpha \\
\hline ILMN_1684982 & PDK4 & -1.25527 & $1.34 \mathrm{E}-05$ & 0.000894 & -5.63735 & Down & pyruvate dehydrogenase kinase 4 \\
\hline ILMN_1791771 & HCK & -0.95686 & $1.35 \mathrm{E}-05$ & 0.000894 & -5.63453 & Down & HCK proto-oncogene, Src family tyrosine kinase \\
\hline ILMN_1712095 & FOXO4 & -0.88154 & $1.36 \mathrm{E}-05$ & 0.000894 & -5.63402 & Down & forkhead box $\mathrm{O} 4$ \\
\hline ILMN_1786034 & GPRC5B & -0.88305 & $1.37 \mathrm{E}-05$ & 0.000898 & -5.63046 & Down & G protein-coupled receptor class $\mathrm{C}$ group 5 member $\mathrm{B}$ \\
\hline ILMN_2216157 & GNA12 & -1.29598 & $1.46 \mathrm{E}-05$ & 0.000933 & -5.60298 & Down & G protein subunit alpha 12 \\
\hline ILMN_1662963 & PSD2 & -1.00118 & $1.47 \mathrm{E}-05$ & 0.000935 & -5.59891 & Down & pleckstrin and Sec7 domain containing 2 \\
\hline ILMN_1769546 & RIN2 & -0.88732 & $1.48 \mathrm{E}-05$ & 0.000935 & -5.59713 & Down & Ras and Rabinteractor 2 \\
\hline ILMN_2128750 & PTTG1IP & -1.12882 & $1.49 \mathrm{E}-05$ & 0.000935 & -5.59436 & Down & PTTG1 interacting protein \\
\hline ILMN_1810275 & SLC7A7 & -1.3872 & $1.5 \mathrm{E}-05$ & 0.000936 & -5.58985 & Down & solute carrier family 7 member 7 \\
\hline ILMN_1658494 & RGCC & -0.85383 & $1.51 \mathrm{E}-05$ & 0.000936 & -5.58791 & Down & regulator of cell cycle \\
\hline ILMN_1675268 & LRP4 & -1.02072 & $1.55 \mathrm{E}-05$ & 0.00095 & -5.5763 & Down & LDL receptor related protein 4 \\
\hline ILMN_1733259 & TMIGD3 & -1.80185 & $1.61 \mathrm{E}-05$ & 0.000962 & -5.55814 & Down & transmembrane and immunoglobulin domain containing 3 \\
\hline ILMN_1765446 & EMP3 & -1.07395 & $1.62 \mathrm{E}-05$ & 0.000962 & -5.55795 & Down & epithelial membrane protein 3 \\
\hline ILMN_2138589 & MERTK & -1.04084 & $1.66 \mathrm{E}-05$ & 0.000967 & -5.54649 & Down & MER proto-oncogene, tyrosine kinase \\
\hline ILMN_2065690 & GRAMD2B & -1.06582 & $1.68 \mathrm{E}-05$ & 0.000972 & -5.54127 & Down & GRAM domain containing 2B \\
\hline ILMN_1815086 & NINJ1 & -1.03546 & $1.68 \mathrm{E}-05$ & 0.000972 & -5.54114 & Down & ninjurin 1 \\
\hline ILMN_1807825 & LY86 & -1.49474 & $1.78 \mathrm{E}-05$ & 0.001009 & -5.51595 & Down & lymphocyte antigen 86 \\
\hline ILMN_1695590 & ADRB2 & -0.92086 & $1.84 \mathrm{E}-05$ & 0.001031 & -5.50231 & Down & adrenoceptor beta 2 \\
\hline ILMN_1723467 & ITGB1 & -1.03672 & $1.85 \mathrm{E}-05$ & 0.001033 & -5.49885 & Down & integrin subunit beta 1 \\
\hline ILMN_1714523 & HEPH & -0.98444 & $1.9 \mathrm{E}-05$ & 0.001048 & -5.48874 & Down & hephaestin \\
\hline ILMN_1659075 & HLA-DOA & -1.06043 & $1.91 \mathrm{E}-05$ & 0.001052 & -5.48585 & Down & major histocompatibility complex, class II, DO alpha \\
\hline ILMN_1670881 & CHST6 & -1.32059 & $1.97 \mathrm{E}-05$ & 0.001056 & -5.47139 & Down & carbohydrate sulfotransferase 6 \\
\hline ILMN_1723481 & CHST3 & -1.13652 & $1.99 \mathrm{E}-05$ & 0.001056 & -5.46859 & Down & carbohydrate sulfotransferase 3 \\
\hline ILMN_1666594 & IRF8 & -1.17618 & $2.05 \mathrm{E}-05$ & 0.001073 & -5.45573 & Down & interferon regulatory factor 8 \\
\hline ILMN_1659895 & MSN & -1.31384 & $2.13 \mathrm{E}-05$ & 0.001089 & -5.43883 & Down & moesin \\
\hline ILMN_2309848 & FXYD5 & -1.13186 & $2.22 \mathrm{E}-05$ & 0.0011 & -5.42098 & Down & FXYD domain containing ion transport regulator 5 \\
\hline ILMN_1744795 & TBL1X & -1.28825 & $2.23 \mathrm{E}-05$ & 0.0011 & -5.42007 & Down & transducin beta like $1 \mathrm{X}$-linked \\
\hline ILMN_1695290 & FERMT2 & -1.25677 & 2.23E-05 & 0.0011 & -5.41852 & Down & fermitin family member 2 \\
\hline ILMN_1682332 & GYPC & -1.35288 & 2.27E-05 & 0.001103 & -5.41156 & Down & glycophorin C (Gerbich blood group) \\
\hline ILMN_1781155 & LYN & -1.40204 & $2.36 \mathrm{E}-05$ & 0.001115 & -5.39504 & Down & LYN proto-oncogene, Src family tyrosine kinase \\
\hline ILMN_2187746 & EMX2 & -1.11174 & $2.36 \mathrm{E}-05$ & 0.001115 & -5.39485 & Down & empty spiracles homeobox 2 \\
\hline ILMN_1786176 & CD37 & -0.87384 & 2.37E-05 & 0.001115 & -5.39366 & Down & CD37 molecule \\
\hline ILMN_1714602 & CD86 & -1.11793 & $2.41 \mathrm{E}-05$ & 0.001124 & -5.386 & Down & CD86 molecule \\
\hline
\end{tabular}




\begin{tabular}{|c|c|c|c|c|c|c|}
\hline ILMN_1752199 & LHPP & -0.88175 & $2.67 \mathrm{E}-05$ & 0.001179 & -5.34225 & Down \\
\hline ILMN_1680652 & SELENBP1 & -0.92212 & $2.69 \mathrm{E}-05$ & 0.001185 & -5.33852 & Down \\
\hline ILMN_1742330 & PLXNB1 & -1.33814 & $2.72 \mathrm{E}-05$ & 0.001192 & -5.3345 & Down \\
\hline ILMN_1682930 & SIPA1 & -1.28477 & $2.74 \mathrm{E}-05$ & 0.001195 & -5.3316 & Down \\
\hline ILMN_1777397 & MSX1 & -1.06783 & $2.78 \mathrm{E}-05$ & 0.001208 & -5.32442 & Down \\
\hline ILMN_1785902 & C1QC & -2.30605 & $2.82 \mathrm{E}-05$ & 0.001211 & -5.31819 & Down \\
\hline ILMN_1660364 & CYTH4 & -1.19865 & $2.82 \mathrm{E}-05$ & 0.001211 & -5.31788 & Down \\
\hline ILMN_2385220 & DFFA & -0.88215 & $2.87 \mathrm{E}-05$ & 0.001222 & -5.31044 & Down \\
\hline ILMN_1659564 & SEC61A1 & -0.87072 & $2.9 \mathrm{E}-05$ & 0.001231 & -5.30663 & Down \\
\hline ILMN_1671565 & RNASET2 & -1.46987 & $2.93 \mathrm{E}-05$ & 0.001236 & -5.30191 & Down \\
\hline ILMN_1738116 & TMEM119 & -1.79973 & $2.99 \mathrm{E}-05$ & 0.001246 & -5.29403 & Down \\
\hline ILMN_1798288 & MOB3C & -1.18371 & $2.99 \mathrm{E}-05$ & 0.001247 & -5.29288 & Down \\
\hline ILMN_1729749 & HERC5 & -1.00142 & $3.02 \mathrm{E}-05$ & 0.00125 & -5.2888 & Down \\
\hline ILMN_1666453 & STK3 & -0.86473 & $3.07 \mathrm{E}-05$ & 0.001256 & -5.28259 & Down \\
\hline ILMN_1754121 & CSK & -0.92241 & $3.13 \mathrm{E}-05$ & 0.001265 & -5.27421 & Down \\
\hline ILMN_2402600 & GLIS3 & -0.89411 & $3.18 \mathrm{E}-05$ & 0.001279 & -5.26738 & Down \\
\hline ILMN_1704537 & PHGDH & -0.93098 & $3.18 \mathrm{E}-05$ & 0.001279 & -5.2673 & Down \\
\hline ILMN_1700432 & ITPKB & -1.63456 & 3.2E-05 & 0.00128 & -5.26388 & Down \\
\hline ILMN_1728426 & INPPL1 & -1.22821 & $3.23 \mathrm{E}-05$ & 0.001287 & -5.26046 & Down \\
\hline ILMN_2330170 & MYBPC1 & -0.95106 & $3.24 \mathrm{E}-05$ & 0.001287 & -5.25976 & Down \\
\hline ILMN_1691572 & TST & -1.0133 & $3.24 \mathrm{E}-05$ & 0.001287 & -5.25867 & Down \\
\hline ILMN_1765578 & TIPARP & -1.09379 & $3.31 \mathrm{E}-05$ & 0.001287 & -5.24996 & Down \\
\hline ILMN_1680925 & SLC9A3R1 & -1.33146 & $3.33 \mathrm{E}-05$ & 0.001293 & -5.24725 & Down \\
\hline ILMN_1749662 & GPX1 & -0.91289 & $3.34 \mathrm{E}-05$ & 0.001295 & -5.24608 & Down \\
\hline ILMN_1660691 & RAB31 & -1.13134 & $3.39 \mathrm{E}-05$ & 0.001307 & -5.23996 & Down \\
\hline ILMN_2242463 & CTSC & -1.0194 & $3.44 \mathrm{E}-05$ & 0.001312 & -5.23389 & Down \\
\hline ILMN_1813561 & SCIN & -1.70847 & $3.5 \mathrm{E}-05$ & 0.001324 & -5.22653 & Down \\
\hline ILMN_1744604 & CYBA & -1.64975 & $3.51 \mathrm{E}-05$ & 0.001324 & -5.22537 & Down \\
\hline ILMN_1668039 & GYPC & -1.42623 & $3.58 \mathrm{E}-05$ & 0.001341 & -5.217 & Down \\
\hline ILMN_1701613 & RARRES3 & -1.41669 & $3.61 \mathrm{E}-05$ & 0.001342 & -5.21315 & Down \\
\hline ILMN_1753547 & STAT5A & -0.89784 & $4.04 \mathrm{E}-05$ & 0.001432 & -5.16499 & Down \\
\hline ILMN_2135258 & RAPGEF3 & -0.98324 & 4.05E-05 & 0.001432 & -5.16378 & Down \\
\hline ILMN_2243687 & LAMP2 & -0.89465 & 4.06E-05 & 0.001432 & -5.16339 & Down \\
\hline ILMN_1731418 & SP110 & -0.85174 & 4.06E-05 & 0.001432 & -5.16328 & Down \\
\hline ILMN_1758672 & FAM107B & -0.97374 & $4.11 \mathrm{E}-05$ & 0.001444 & -5.15776 & Down \\
\hline ILMN_1699931 & HCST & -1.58721 & $4.16 \mathrm{E}-05$ & 0.001444 & -5.15308 & Down \\
\hline ILMN_2366330 & FERMT3 & -1.08457 & 4.17E-05 & 0.001444 & -5.15152 & Down \\
\hline ILMN_1715715 & CEBPA & -1.49784 & 4.17E-05 & 0.001444 & -5.15133 & Down \\
\hline ILMN_1803560 & LAT2 & -1.18665 & 4.27E-05 & 0.001459 & -5.14176 & Down \\
\hline ILMN_1727043 & COLGALT1 & -0.8402 & $4.31 \mathrm{E}-05$ & 0.001465 & -5.13784 & Do \\
\hline ILMN_1741165 & SLC11A1 & -1.59253 & $4.41 \mathrm{E}-05$ & 0.001483 & -5.12822 & Down \\
\hline ILMN_1747744 & LHFPL2 & -0.99133 & 4.42E-05 & 0.001483 & -5.12745 & Down \\
\hline
\end{tabular}

phospholysinephosphohistidine inorganic pyrophosphate phosphatase

selenium binding protein 1 plexin B1

signal-induced proliferation-associated 1 mshhomeobox 1 complement $\mathrm{C} 1 \mathrm{q} \mathrm{C}$ chain cytohesin 4

DNA fragmentation factor subunit alpha

Sec61 translocon alpha 1 subunit ribonuclease $\mathrm{T} 2$

transmembrane protein 119

MOB kinase activator $3 \mathrm{C}$

HECT and RLD domain containing E3 ubiquitin protein ligase 5

serine/threonine kinase 3

C-terminal Src kinase

GLIS family zinc finger 3

\section{phosphoglycerate dehydrogenase}

inositol-trisphosphate 3-kinase B

inositol polyphosphate phosphatase like 1

myosin binding protein $\mathrm{C}$, slow type

thiosulfate sulfurtransferase

TCDD inducible poly(ADP-ribose) polymerase

SLC9A3 regulator 1

glutathione peroxidase 1

RAB31, member RAS oncogene family

$$
\text { cathepsin C }
$$

scinderin

cytochrome b-245 alpha chain

glycophorin C (Gerbich blood group)

retinoic acid receptor responder 3

signal transducer and activator of transcription $5 \mathrm{~A}$

Rap guanine nucleotide exchange factor 3

lysosomal associated membrane protein 2

SP110 nuclear body protein

family with sequence similarity 107 member B

hematopoietic cell signal transducer

fermitin family member 3

CCAAT enhancer binding protein alpha

linker for activation of $\mathrm{T}$ cells family member 2

collagen beta(1-O)galactosyltransferase 1

solute carrier family 11 member 1

LHFPL tetraspan subfamily member 2 
medRxiv preprint doi: https://doi.org/10.1101/2020.12.21.20248688; this version posted December 24, 2020. The copyright holder for this preprint (which was not certified by peer review) is the author/funder, who has granted medRxiv a license to display the preprint in perpetuity. All rights reserved. No reuse allowed without permission.

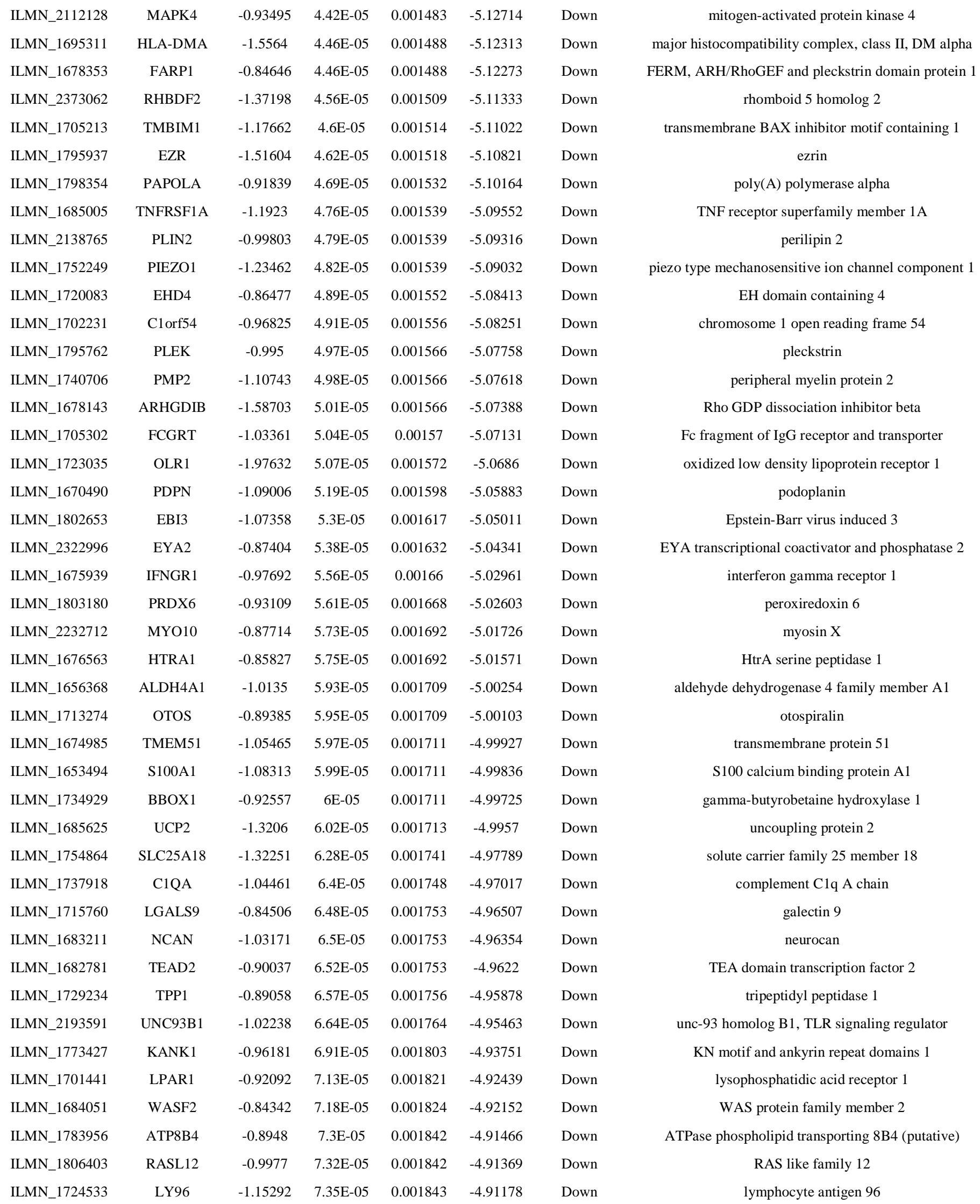




\begin{tabular}{|c|c|c|c|c|c|c|c|}
\hline ILMN_1697554 & SASH3 & -0.84241 & $7.51 \mathrm{E}-05$ & 0.001857 & -4.90272 & Down & SAM and SH3 domain containing 3 \\
\hline ILMN_1698766 & PYCARD & -1.12399 & $7.8 \mathrm{E}-05$ & 0.00191 & -4.88655 & Down & $\begin{array}{l}\text { PYD and CARD domain containing } \\
\text { amyloid beta precursor protein binding family B member } 1\end{array}$ \\
\hline ILMN_1801710 & APBB1IP & -1.24443 & $7.86 \mathrm{E}-05$ & 0.001916 & -4.88355 & Down & interacting protein \\
\hline ILMN_1802167 & ALDH1L1 & -1.50879 & 8.03E-05 & 0.001942 & -4.87455 & Down & aldehyde dehydrogenase 1 family member L1 \\
\hline ILMN_1675848 & MYL12A & -0.94028 & $8.14 \mathrm{E}-05$ & 0.001955 & -4.86849 & Down & myosin light chain $12 \mathrm{~A}$ \\
\hline ILMN_1797875 & ALOX5AP & -1.38325 & $8.29 \mathrm{E}-05$ & 0.001983 & -4.86085 & Down & arachidonate 5-lipoxygenase activating protein \\
\hline ILMN_1655549 & SIGLEC10 & -1.30448 & $8.38 \mathrm{E}-05$ & 0.001992 & -4.85644 & Down & sialic acid binding Ig like lectin 10 \\
\hline ILMN_1794017 & SERTAD1 & -0.85735 & $8.56 \mathrm{E}-05$ & 0.002011 & -4.84748 & Down & SERTA domain containing 1 \\
\hline ILMN_1723480 & BST2 & -1.27529 & $8.56 \mathrm{E}-05$ & 0.002011 & -4.84748 & Down & bone marrow stromal cell antigen 2 \\
\hline ILMN_1727402 & HCLS1 & -1.37672 & $8.6 \mathrm{E}-05$ & 0.002017 & -4.84541 & Down & hematopoietic cell-specific Lyn substrate 1 \\
\hline ILMN_1694589 & PAQR8 & -1.11775 & $8.65 \mathrm{E}-05$ & 0.002024 & -4.84325 & Down & progestin and adipoQ receptor family member 8 \\
\hline ILMN_1720850 & BAZ2B & -0.89055 & $8.71 \mathrm{E}-05$ & 0.002028 & -4.84011 & Down & bromodomain adjacent to zinc finger domain $2 \mathrm{~B}$ \\
\hline ILMN_2085862 & SLC15A3 & -1.32691 & $8.75 \mathrm{E}-05$ & 0.002028 & -4.83815 & Down & solute carrier family 15 member 3 \\
\hline ILMN_1756595 & SH3TC1 & -0.89823 & $8.8 \mathrm{E}-05$ & 0.002028 & -4.83596 & Down & SH3 domain and tetratricopeptide repeats 1 \\
\hline ILMN_1763000 & ADAP2 & -1.03912 & 8.83E-05 & 0.002028 & -4.83418 & Down & ArfGAP with dual PH domains 2 \\
\hline ILMN_2404688 & NUPR1 & -0.89176 & 8.88E-05 & 0.002034 & -4.83207 & Down & nuclear protein 1 , transcriptional regulator \\
\hline ILMN_1726981 & VEGFB & -0.93446 & $9.06 \mathrm{E}-05$ & 0.002056 & -4.82346 & Down & vascular endothelial growth factor B \\
\hline ILMN_2412214 & LGALS9 & -0.84754 & $9.31 \mathrm{E}-05$ & 0.002091 & -4.81221 & Down & galectin 9 \\
\hline ILMN_1693552 & CD300A & -1.04773 & $9.34 \mathrm{E}-05$ & 0.002091 & -4.81093 & Down & CD300a molecule \\
\hline ILMN_1663640 & MAOA & -0.97927 & $9.36 \mathrm{E}-05$ & 0.002092 & -4.80993 & Down & monoamine oxidase $\mathrm{A}$ \\
\hline ILMN_1670379 & ANTXR1 & -1.21747 & $9.45 \mathrm{E}-05$ & 0.002106 & -4.80568 & Down & ANTXR cell adhesion molecule 1 \\
\hline ILMN_2157441 & HLA-DRA & -1.86831 & $9.59 \mathrm{E}-05$ & 0.00212 & -4.79956 & Down & major histocompatibility complex, class II, DR alpha \\
\hline ILMN_2244841 & ALDH4A1 & -0.91264 & $9.59 \mathrm{E}-05$ & 0.00212 & -4.79947 & Down & aldehyde dehydrogenase 4 family member A1 \\
\hline ILMN_1671337 & SLC2A5 & -1.38535 & $9.61 \mathrm{E}-05$ & 0.00212 & -4.79851 & Down & solute carrier family 2 member 5 \\
\hline ILMN_1691717 & RHBDF2 & -1.34499 & $9.88 \mathrm{E}-05$ & 0.002147 & -4.78703 & Down & rhomboid 5 homolog 2 \\
\hline ILMN_1738675 & PTPN6 & -1.10539 & 0.000103 & 0.002193 & -4.77006 & Down & protein tyrosine phosphatase, non-receptor type 6 \\
\hline ILMN_2129234 & TMEM47 & -1.17592 & 0.000103 & 0.002196 & -4.76807 & Down & transmembrane protein 47 \\
\hline ILMN_1756982 & CLIC1 & -1.06088 & 0.000104 & 0.002196 & -4.76713 & Down & chloride intracellular channel 1 \\
\hline ILMN_1782439 & CNN3 & -1.31016 & 0.000104 & 0.0022 & -4.76528 & Down & calponin 3 \\
\hline ILMN_2241775 & TROVE2 & -0.93526 & 0.000106 & 0.002226 & -4.75674 & Down & TROVE domain family member 2 \\
\hline ILMN_2359907 & CD68 & -1.40686 & 0.000106 & 0.002229 & -4.75586 & Down & CD68 molecule \\
\hline ILMN_1677432 & SRGAP1 & -1.03784 & 0.000107 & 0.002229 & -4.75483 & Down & SLIT-ROBO Rho GTPase activating protein 1 \\
\hline ILMN_1655987 & STAB1 & -1.01047 & 0.000108 & 0.002248 & -4.74782 & Down & stabilin 1 \\
\hline ILMN_1687840 & ABCB7 & -0.91909 & 0.00011 & 0.002248 & -4.74282 & Down & ATP binding cassette subfamily B member 7 \\
\hline ILMN_2297765 & KCNMA1 & -0.90411 & 0.00011 & 0.002248 & -4.74232 & Down & potassium calcium-activated channel subfamily $\mathrm{M}$ alpha 1 \\
\hline ILMN_1752269 & ACSS1 & -1.08814 & 0.000111 & 0.002257 & -4.73795 & Down & acyl-CoA synthetase short chain family member 1 \\
\hline ILMN_1768505 & IL13RA1 & -1.11483 & 0.000113 & 0.002272 & -4.73071 & Down & interleukin 13 receptor subunit alpha 1 \\
\hline ILMN_1736178 & AEBP1 & -1.10127 & 0.000113 & 0.002278 & -4.72896 & Down & AE binding protein 1 \\
\hline ILMN_1738552 & SLC1A3 & -1.38109 & 0.000114 & 0.002278 & -4.72806 & Down & solute carrier family 1 member 3 \\
\hline ILMN_1655154 & PTBP1 & -1.12111 & 0.000115 & 0.002297 & -4.72199 & Down & polypyrimidine tract binding protein 1 \\
\hline ILMN_1687508 & ALDH7A1 & -0.90537 & 0.000116 & 0.002308 & -4.71848 & Down & aldehyde dehydrogenase 7 family member A1 \\
\hline ILMN_1809467 & VAMP5 & -0.95918 & 0.000118 & 0.002327 & -4.71238 & Down & vesicle associated membrane protein 5 \\
\hline
\end{tabular}




\begin{tabular}{|c|c|c|c|c|c|c|c|}
\hline ILMN_2364022 & SLC16A3 & -1.12445 & 0.00012 & 0.002342 & -4.70661 & Down & solute carrier family 16 member 3 \\
\hline ILMN_1761733 & HLA-DMB & -1.80048 & 0.000121 & 0.002358 & -4.7008 & Down & major histocompatibility complex, class II, DM beta \\
\hline ILMN_1703330 & FEM1C & -0.84819 & 0.000122 & 0.002369 & -4.6967 & Down & $\begin{array}{c}\text { fem-1 homolog C } \\
\text { ArfGAP with SH3 domain, ankyrin repeat and PH domain }\end{array}$ \\
\hline ILMN_1685441 & ASAP3 & -0.89215 & 0.000123 & 0.002376 & -4.69383 & Down & 3 \\
\hline ILMN_1686623 & CSF1R & -1.66221 & 0.000125 & 0.002387 & -4.68834 & Down & colony stimulating factor 1 receptor \\
\hline ILMN_1712400 & SERPINB6 & -0.98311 & 0.000129 & 0.002447 & -4.67353 & Down & serpin family B member 6 \\
\hline ILMN_1701025 & EPHX1 & -1.10209 & 0.000129 & 0.002447 & -4.67347 & Down & epoxide hydrolase 1 \\
\hline ILMN_1751851 & $\mathrm{ADA} 2$ & -1.02455 & 0.00013 & 0.002456 & -4.67157 & Down & adenosine deaminase 2 \\
\hline ILMN_1808587 & ZFHX3 & -0.86205 & 0.000131 & 0.002459 & -4.66911 & Down & zinc finger homeobox 3 \\
\hline ILMN_2179717 & FAM189A2 & -0.94927 & 0.000136 & 0.002505 & -4.65429 & Down & family with sequence similarity 189 member A2 \\
\hline ILMN_1659766 & BAG3 & -1.48902 & 0.000136 & 0.002511 & -4.65208 & Down & BCL2 associated athanogene 3 \\
\hline ILMN_1709747 & EXOG & -1.22546 & 0.000137 & 0.002515 & -4.64942 & Down & exo/endonuclease $\mathrm{G}$ \\
\hline ILMN_2374449 & SPP1 & -1.66792 & 0.000137 & 0.002515 & -4.64851 & Down & secreted phosphoprotein 1 \\
\hline ILMN_1747499 & EMID1 & -0.86351 & 0.000151 & 0.002654 & -4.60875 & Down & EMI domain containing 1 \\
\hline ILMN_2302757 & FCGBP & -2.34218 & 0.000151 & 0.002654 & -4.60857 & Down & $\mathrm{Fc}$ fragment of $\operatorname{IgG}$ binding protein \\
\hline ILMN_1741632 & RAB3IL1 & -0.84802 & 0.000152 & 0.002655 & -4.60682 & Down & RAB3A interacting protein like 1 \\
\hline ILMN_1751079 & TAP1 & -0.93331 & 0.000155 & 0.002691 & -4.59882 & Down & transporter 1 , ATP binding cassette subfamily B member \\
\hline ILMN_1744949 & RHOBTB3 & -1.01304 & 0.000159 & 0.00274 & -4.58655 & Down & Rho related BTB domain containing 3 \\
\hline ILMN_1794677 & TMC6 & -0.86609 & 0.000161 & 0.002766 & -4.5808 & Down & transmembrane channel like 6 \\
\hline ILMN_1701195 & PLA2G7 & -0.89751 & 0.000165 & 0.002801 & -4.5721 & Down & phospholipase A2 group VII \\
\hline ILMN_1789244 & SOX8 & -0.86327 & 0.000168 & 0.002821 & -4.56529 & Down & SRY-box 8 \\
\hline ILMN_2205963 & VSIR & -1.21173 & 0.000168 & 0.002825 & -4.56423 & Down & V-set immunoregulatory receptor \\
\hline ILMN_1737314 & BCL6 & -1.03335 & 0.000171 & 0.002854 & -4.55684 & Down & B cell CLL/lymphoma 6 \\
\hline ILMN_1704398 & FZD9 & -0.93759 & 0.000175 & 0.002887 & -4.54601 & Down & frizzled class receptor 9 \\
\hline ILMN_1740938 & APOE & -1.39818 & 0.000176 & 0.002892 & -4.545 & Down & apolipoprotein E \\
\hline ILMN_1766657 & STOM & -1.07331 & 0.000179 & 0.002926 & -4.53871 & Down & stomatin \\
\hline ILMN_2146389 & MEGF10 & -0.95815 & 0.000182 & 0.002955 & -4.53048 & Down & multiple EGF like domains 10 \\
\hline ILMN_1712708 & TRIM47 & -1.01888 & 0.000188 & 0.003007 & -4.51674 & Down & tripartite motif containing 47 \\
\hline ILMN_1732410 & SLC16A9 & -1.25039 & 0.000197 & 0.003095 & -4.4972 & Down & solute carrier family 16 member 9 \\
\hline ILMN_1739792 & RHOG & -0.86338 & 0.000198 & 0.003103 & -4.49448 & Down & ras homolog family member $\mathrm{G}$ \\
\hline ILMN_1736567 & CD74 & -1.62418 & 0.000198 & 0.003103 & -4.49443 & Down & CD74 molecule \\
\hline ILMN_1759023 & WFS1 & -0.97166 & 0.0002 & 0.003123 & -4.49039 & Down & wolframin ER transmembrane glycoprotein \\
\hline ILMN_1766054 & ABCA1 & -1.55785 & 0.000203 & 0.003144 & -4.48516 & Down & ATP binding cassette subfamily A member 1 \\
\hline ILMN_1672536 & FBLN1 & -0.88096 & 0.000206 & 0.003173 & -4.47915 & Down & fibulin 1 \\
\hline ILMN_2326713 & CD151 & -0.91821 & 0.000206 & 0.003173 & -4.47873 & Down & CD151 molecule (Raph blood group) \\
\hline ILMN_2379644 & CD74 & -1.46615 & 0.000212 & 0.003228 & -4.46606 & Down & CD74 molecule \\
\hline ILMN_1660125 & SFMBT2 & -1.42268 & 0.000215 & 0.00325 & -4.46069 & Down & Scm like with four mbt domains 2 \\
\hline ILMN_2352097 & ADGRG1 & -1.12033 & 0.00022 & 0.003296 & -4.45227 & Down & adhesion $\mathrm{G}$ protein-coupled receptor $\mathrm{G} 1$ \\
\hline ILMN_1780465 & CLEC5A & -0.85234 & 0.00022 & 0.003301 & -4.45062 & Down & C-type lectin domain containing 5A \\
\hline ILMN_1688886 & GPC5 & -1.08779 & 0.000221 & 0.003304 & -4.44895 & Down & glypican 5 \\
\hline ILMN_1782050 & CEBPD & -1.1621 & 0.000228 & 0.003364 & -4.43557 & Down & CCAAT enhancer binding protein delta \\
\hline ILMN_1680344 & MYOM1 & -1.00101 & 0.000229 & 0.003364 & -4.43513 & Down & myomesin 1 \\
\hline
\end{tabular}




\begin{tabular}{|c|c|c|c|c|c|c|c|}
\hline ILMN_2333319 & PTBP1 & -1.01501 & 0.000229 & 0.003364 & -4.43502 & Down & polypyrimidine tract binding protein 1 \\
\hline ILMN_2384122 & ADGRG1 & -1.11724 & 0.00023 & 0.003365 & -4.43314 & Down & adhesion G protein-coupled receptor G1 \\
\hline ILMN_1762696 & FAM181A & -1.19022 & 0.00023 & 0.003365 & -4.43293 & Down & family with sequence similarity 181 member A \\
\hline ILMN_1788874 & SERPINA3 & -3.16742 & 0.000231 & 0.003365 & -4.43133 & Down & serpin family A member 3 \\
\hline ILMN_2334210 & ITGB4 & -1.06675 & 0.000232 & 0.003371 & -4.42892 & Down & integrin subunit beta 4 \\
\hline ILMN_1782944 & GPR37L1 & -1.11851 & 0.000238 & 0.003431 & -4.41783 & Down & G protein-coupled receptor 37 like 1 \\
\hline ILMN_1760027 & WAS & -1.09035 & 0.000249 & 0.003518 & -4.39997 & Down & Wiskott-Aldrich syndrome \\
\hline ILMN_1794803 & NDP & -0.88575 & 0.000249 & 0.003518 & -4.39991 & Down & NDP, norrincystine knot growth factor \\
\hline ILMN_2144426 & HIST2H2AA3 & -0.85173 & 0.000261 & 0.003605 & -4.37951 & Down & histone cluster $2 \mathrm{H} 2 \mathrm{~A}$ family member a3 \\
\hline ILMN_2176063 & FCGR1A & -1.00902 & 0.000264 & 0.003617 & -4.37548 & Down & $\mathrm{Fc}$ fragment of $\mathrm{IgG}$ receptor Ia \\
\hline ILMN_2286987 & BTBD17 & -1.03886 & 0.000264 & 0.003623 & -4.37436 & Down & BTB domain containing 17 \\
\hline ILMN_1742618 & XAF1 & -1.14564 & 0.000269 & 0.003661 & -4.3674 & Down & XIAP associated factor 1 \\
\hline ILMN_1703123 & CSRNP1 & -0.92513 & 0.000272 & 0.003685 & -4.36326 & Down & cysteine and serine rich nuclear protein 1 \\
\hline ILMN_1691156 & MT1A & -1.28131 & 0.000284 & 0.003788 & -4.34511 & Down & metallothionein $1 \mathrm{~A}$ \\
\hline ILMN_1767337 & SFXN5 & -0.93232 & 0.000291 & 0.003843 & -4.33435 & Down & sideroflexin 5 \\
\hline ILMN_1780057 & RENBP & -0.99772 & 0.000292 & 0.003854 & -4.33229 & Down & renin binding protein \\
\hline ILMN_1714067 & NTRK2 & -1.15825 & 0.000304 & 0.003941 & -4.31617 & Down & neurotrophic receptor tyrosine kinase 2 \\
\hline ILMN_1721540 & NWD1 & -1.04035 & 0.000304 & 0.003942 & -4.31569 & Down & NACHT and WD repeat domain containing 1 \\
\hline ILMN_1792473 & AIF1 & -1.32938 & 0.000309 & 0.003974 & -4.30999 & Down & allograft inflammatory factor 1 \\
\hline ILMN_1728049 & S100A16 & -0.85785 & 0.00031 & 0.003983 & -4.30845 & Down & S100 calcium binding protein A16 \\
\hline ILMN_1688480 & CCND1 & -1.03472 & 0.000313 & 0.004004 & -4.30452 & Down & cyclin D1 \\
\hline ILMN_2254635 & ITGAX & -1.38076 & 0.000314 & 0.004015 & -4.30255 & Down & integrin subunit alpha $X$ \\
\hline ILMN_1736670 & PPP1R3C & -1.32738 & 0.000325 & 0.004106 & -4.28867 & Down & protein phosphatase 1 regulatory subunit $3 \mathrm{C}$ \\
\hline ILMN_1772218 & HLA-DPA1 & -1.31052 & 0.000335 & 0.004169 & -4.27599 & Down & major histocompatibility complex, class II, DP alpha 1 \\
\hline ILMN_1813639 & NKAIN4 & -0.86855 & 0.000338 & 0.004185 & -4.27219 & Down & sodium/potassium transporting ATPase interacting 4 \\
\hline ILMN_1733538 & RGS10 & -0.86955 & 0.000339 & 0.004185 & -4.27112 & Down & regulator of $\mathrm{G}$ protein signaling 10 \\
\hline ILMN_1775330 & CCDC9B & -0.84316 & 0.000351 & 0.004256 & -4.25639 & Down & coiled-coil domain containing 9B \\
\hline ILMN_2371280 & CSF3R & -0.90393 & 0.000352 & 0.00426 & -4.25504 & Down & colony stimulating factor 3 receptor \\
\hline ILMN_1696839 & RPS27 & -0.84631 & 0.000352 & 0.00426 & -4.25489 & Down & ribosomal protein S27 \\
\hline ILMN_1686664 & MT2A & -1.35351 & 0.000353 & 0.004265 & -4.25418 & Down & metallothionein $2 \mathrm{~A}$ \\
\hline ILMN_2259119 & PRMT2 & -0.92999 & 0.000355 & 0.004284 & -4.2516 & Down & protein arginine methyltransferase 2 \\
\hline ILMN_1797693 & BRI3BP & -0.84114 & 0.000357 & 0.004304 & -4.24878 & Down & BRI3 binding protein \\
\hline ILMN_1760890 & SELENON & -0.84534 & 0.000358 & 0.00431 & -4.24753 & Down & $\begin{array}{l}\text { selenoprotein } \mathrm{N} \\
\text { potassium calcium-activated channel subfamily } \mathrm{N} \text { member }\end{array}$ \\
\hline ILMN_1744897 & KCNN3 & -0.98378 & 0.000377 & 0.004467 & -4.22597 & Down & 3 \\
\hline ILMN_1736184 & GSTM3 & -0.85687 & 0.000377 & 0.004467 & -4.22593 & Down & glutathione S-transferase mu 3 \\
\hline ILMN_1697176 & GFAP & -1.71446 & 0.00038 & 0.004489 & -4.22326 & Down & glial fibrillary acidic protein \\
\hline ILMN_1732154 & BCAN & -0.85376 & 0.000386 & 0.004527 & -4.21691 & Down & brevican \\
\hline ILMN_1663042 & SDC4 & -1.50698 & 0.000387 & 0.004539 & -4.215 & Down & syndecan 4 \\
\hline ILMN_1768101 & HOXB6 & -0.92457 & 0.000398 & 0.004613 & -4.20347 & Down & homeobox B6 \\
\hline ILMN_1802923 & APOC2 & -1.07369 & 0.000404 & 0.004645 & -4.1977 & Down & apolipoprotein $\mathrm{C} 2$ \\
\hline ILMN_1680996 & ALOX5 & -0.91327 & 0.000414 & 0.004725 & -4.18672 & Down & arachidonate 5-lipoxygenase \\
\hline ILMN_1726388 & ACBD7 & -1.0819 & 0.000425 & 0.004792 & -4.17607 & Down & acyl-CoA binding domain containing 7 \\
\hline
\end{tabular}




\begin{tabular}{|c|c|c|c|c|c|c|c|}
\hline ILMN_1769394 & PLCD1 & -0.8429 & 0.000427 & 0.004804 & -4.17378 & Down & phospholipase $\mathrm{C}$ delta 1 \\
\hline ILMN_1686679 & ZNF462 & -0.86512 & 0.000429 & 0.004812 & -4.17223 & Down & zinc finger protein 462 \\
\hline ILMN_1744487 & MFRP & -0.87517 & 0.000433 & 0.004838 & -4.16858 & Down & membrane frizzled-related protein \\
\hline ILMN_1718063 & LIPA & -0.8547 & 0.000447 & 0.004938 & -4.15557 & Down & lipase A, lysosomal acid type \\
\hline ILMN_1803811 & TRIB1 & -0.94381 & 0.000448 & 0.004942 & -4.15431 & Down & tribblespseudokinase 1 \\
\hline ILMN_1730229 & CGNL1 & -0.9493 & 0.00046 & 0.005031 & -4.14294 & Down & cingulin like 1 \\
\hline ILMN_2136089 & MT1IP & -1.0151 & 0.000465 & 0.005061 & -4.1382 & Down & metallothionein 1I, pseudogene \\
\hline ILMN_1753182 & COL20A1 & -0.84268 & 0.000488 & 0.005189 & -4.11881 & Down & collagen type XX alpha 1 chain \\
\hline ILMN_2115490 & NBPF9 & -1.26164 & 0.000504 & 0.005285 & -4.10505 & Down & NBPF member 9 \\
\hline ILMN_1727087 & GJA1 & -1.27222 & 0.000509 & 0.005312 & -4.10061 & Down & gap junction protein alpha 1 \\
\hline ILMN_2128428 & DAB2 & -1.08238 & 0.000513 & 0.005325 & -4.09779 & Down & DAB2, clathrin adaptor protein \\
\hline ILMN_1784884 & LILRB3 & -0.84783 & 0.000518 & 0.005373 & -4.09335 & Down & leukocyte immunoglobulin like receptor B3 \\
\hline ILMN_1763852 & ACACB & -0.98693 & 0.000528 & 0.005442 & -4.08517 & Down & acetyl-CoA carboxylase beta \\
\hline ILMN_1709795 & $\mathrm{RAC} 2$ & -0.8545 & 0.000544 & 0.005563 & -4.07267 & Down & Rac family small GTPase 2 \\
\hline ILMN_1702698 & LSM11 & -0.95097 & 0.000552 & 0.005614 & -4.06676 & Down & LSM11, U7 small nuclear RNA associated \\
\hline ILMN_1725696 & ATXN3 & -1.28811 & 0.000561 & 0.005671 & -4.06011 & Down & $\operatorname{ataxin} 3$ \\
\hline ILMN_1753143 & RHPN2 & -1.09171 & 0.000563 & 0.005681 & -4.05835 & Down & rhophilin Rho GTPase binding protein 2 \\
\hline ILMN_1714567 & AHNAK & -1.01719 & 0.000568 & 0.00571 & -4.05503 & Down & AHNAK nucleoprotein \\
\hline ILMN_1803429 & CD44 & -1.48233 & 0.00057 & 0.005716 & -4.05346 & Down & CD44 molecule (Indian blood group) \\
\hline ILMN_2384056 & GPER1 & -0.93709 & 0.000596 & 0.005862 & -4.03489 & Down & G protein-coupled estrogen receptor 1 \\
\hline ILMN_1779706 & ТР53ВР2 & -0.9149 & 0.000601 & 0.005894 & -4.0316 & Down & $\begin{array}{l}\text { tumor protein } \mathrm{p} 53 \text { binding protein } 2 \\
\text { LIM domain containing preferred translocation partner in }\end{array}$ \\
\hline ILMN_1651254 & LPP & -0.97791 & 0.000631 & 0.006073 & -4.0113 & Down & lipoma \\
\hline ILMN_2360291 & UGGT1 & -0.98566 & 0.000657 & 0.006231 & -3.99441 & Down & UDP-glucose glycoprotein glucosyltransferase 1 \\
\hline ILMN_1775380 & SMOX & -0.85447 & 0.000683 & 0.006406 & -3.97814 & Down & spermine oxidase \\
\hline ILMN_2206746 & BGN & -1.10183 & 0.00072 & 0.006632 & -3.95603 & Down & biglycan \\
\hline ILMN_1737650 & $\mathrm{DIO} 2$ & -1.33173 & 0.000745 & 0.006784 & -3.94172 & Down & iodothyroninedeiodinase 2 \\
\hline ILMN_1656011 & RGS1 & -1.07966 & 0.000754 & 0.006828 & -3.93674 & Down & regulator of $\mathrm{G}$ protein signaling 1 \\
\hline ILMN_2396444 & CD14 & -1.7274 & 0.000768 & 0.006918 & -3.92883 & Down & CD14 molecule \\
\hline ILMN_2227790 & GRIN2C & -1.33411 & 0.000795 & 0.007052 & -3.91424 & Down & glutamate ionotropic receptor NMDA type subunit 2C \\
\hline ILMN_2128795 & LRIG1 & -1.02318 & 0.000814 & 0.007156 & -3.90424 & Down & leucine rich repeats and immunoglobulin like domains 1 \\
\hline ILMN_1697793 & SYNJ2BP & -0.88542 & 0.000817 & 0.00717 & -3.9028 & Down & synaptojanin 2 binding protein \\
\hline ILMN_2379599 & CD163 & -1.28796 & 0.000853 & 0.007373 & -3.88498 & Down & CD163 molecule \\
\hline ILMN_1740015 & $\mathrm{CD} 14$ & -1.44122 & 0.00087 & 0.007454 & -3.87653 & Down & $\begin{array}{l}\text { CD14 molecule } \\
\text { solute carrier organic anion transporter family member }\end{array}$ \\
\hline ILMN_2087656 & SLCO2B1 & -1.08271 & 0.000923 & 0.007739 & -3.85189 & Down & $2 \mathrm{~B} 1$ \\
\hline ILMN_1814526 & ADD3 & -0.91359 & 0.000931 & 0.007774 & -3.84818 & Down & adducin 3 \\
\hline ILMN_2306540 & PDE9A & -0.91804 & 0.000981 & 0.008072 & -3.82612 & Down & phosphodiesterase $9 \mathrm{~A}$ \\
\hline ILMN_1772644 & EML3 & -0.95437 & 0.001028 & 0.008302 & -3.80649 & Down & echinoderm microtubule associated protein like 3 \\
\hline ILMN_1707727 & ANGPTL4 & -1.57156 & 0.001041 & 0.00836 & -3.80145 & Down & angiopoietin like 4 \\
\hline ILMN_2121068 & ADAM17 & -0.88252 & 0.00105 & 0.008417 & -3.79783 & Down & ADAM metallopeptidase domain 17 \\
\hline ILMN_1747271 & ATP1B2 & -0.93591 & 0.001093 & 0.008639 & -3.78095 & Down & ATPase $\mathrm{Na}+/ \mathrm{K}+$ transporting subunit beta 2 \\
\hline ILMN_1754894 & C1orf162 & -1.14419 & 0.001117 & 0.008772 & -3.77161 & Down & chromosome 1 open reading frame 162 \\
\hline ILMN_2227011 & ACSBG1 & -1.22586 & 0.00121 & 0.009246 & -3.73812 & Down & acyl-CoA synthetasebubblegum family member 1 \\
\hline
\end{tabular}




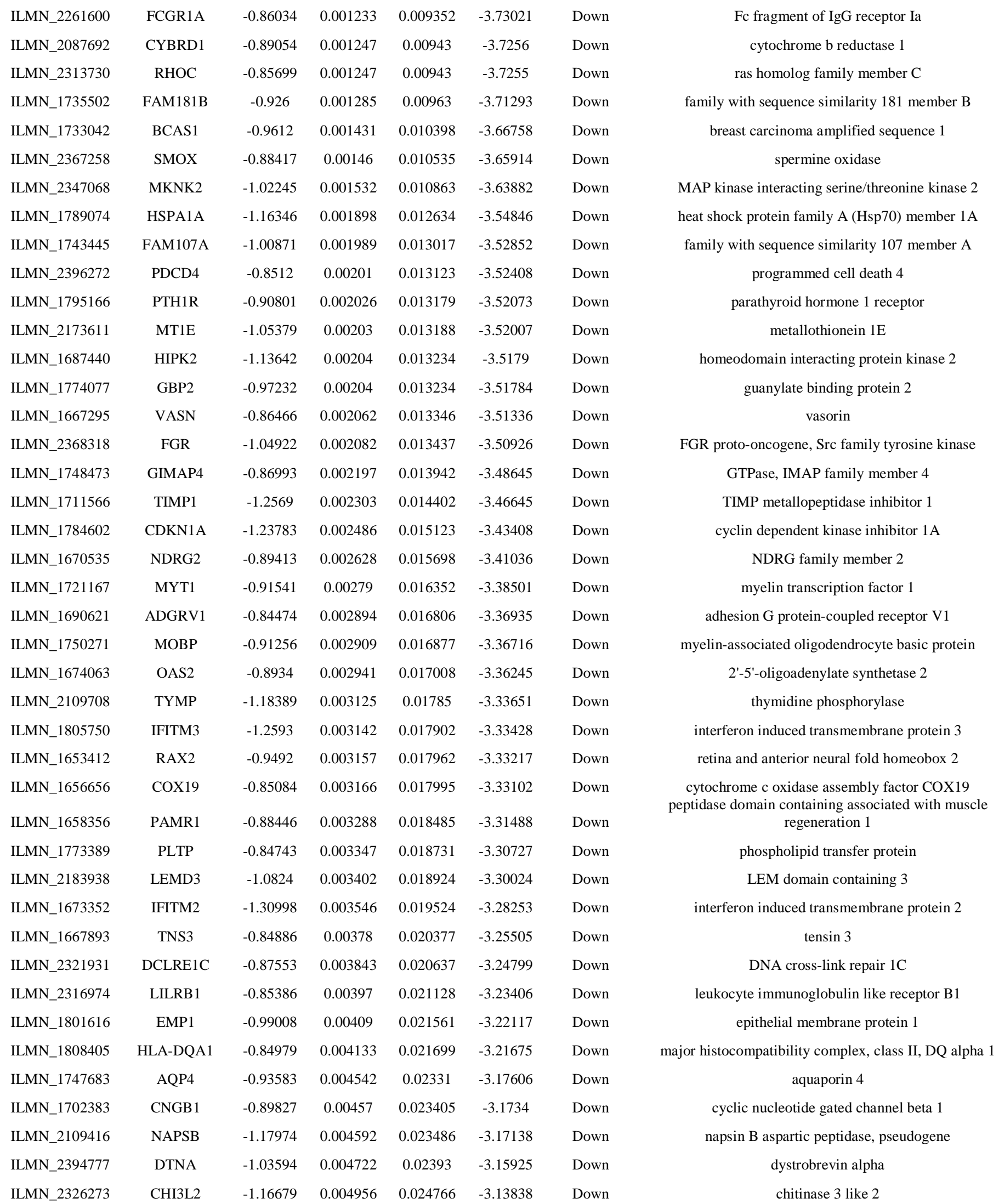


medRxiv preprint doi: https://doi.org/10.1101/2020.12.21.20248688; this version posted December 24, 2020. The copyright holder for this preprint (which was not certified by peer review) is the author/funder, who has granted medRxiv a license to display the preprint in perpetuity.

All rights reserved. No reuse allowed without permission.

$\begin{array}{lccccccc}\text { ILMN_1775170 } & \text { MT1X } & -1.19236 & 0.004958 & 0.024767 & -3.13818 & \text { Down } & \text { metallothionein 1X } \\ \text { ILMN_1652549 } & \text { DTNA } & -1.00054 & 0.005029 & 0.025005 & -3.13204 & \text { Down } & \text { dystrobrevin alpha } \\ \text { ILMN_1680110 } & \text { ADIRF } & -1.15131 & 0.005636 & 0.027171 & -3.08264 & \text { Down } & \text { adipogenesis regulatory factor } \\ \text { ILMN_2376458 } & \text { CSF2RA } & -0.93258 & 0.005641 & 0.027181 & -3.08227 & \text { Down } & \text { colony stimulating factor 2 receptor alpha subunit } \\ \text { ILMN_2201580 } & \text { \#N/A } & -0.94087 & 0.005782 & 0.027669 & -3.07156 & \text { Down } & \text { spectrin beta, non-erythrocytic 1 } \\ \text { ILMN_1661335 } & \text { SPTBN1 } & -0.86298 & 0.006971 & 0.031749 & -2.98998 & \text { Down } & \text { TNF superfamily member 14 } \\ \text { ILMN_2363392 } & \text { TNFSF14 } & -0.85388 & 0.007499 & 0.033469 & -2.95798 & \text { Down } & \text { fibroblast growth factor receptor 3 } \\ \text { ILMN_1723123 } & \text { FGFR3 } & -0.8616 & 0.007561 & 0.03368 & -2.95435 & \text { Down } & \text { metallothionein 1M } \\ \text { ILMN_1657435 } & \text { MT1M } & -0.87928 & 0.010473 & 0.042682 & -2.81032 & \text { Down } & \text { apelin receptor } \\ \text { ILMN_1700183 } & \text { APLNR } & -1.08822 & 0.011405 & 0.045366 & -2.77227 & \text { Down } & \end{array}$

Table 2 The enriched pathway terms of the up regulated differentially expressed genes

BIOCYC

\begin{tabular}{|c|c|c|c|c|c|c|c|}
\hline Pathway ID & Pathway Name & P-value & $\begin{array}{l}\text { FDR } \\
\text { B\&H }\end{array}$ & $\begin{array}{l}\text { FDR } \\
\text { B\&Y }\end{array}$ & $\begin{array}{c}\text { Bonferr } \\
\text { oni }\end{array}$ & $\begin{array}{c}\text { Gen } \\
\text { e } \\
\text { Cou } \\
\text { nt }\end{array}$ & Gene \\
\hline 547502 & $\begin{array}{c}\text { glutamine } \\
\text { degradation/glutam } \\
\text { ate biosynthesis }\end{array}$ & $4.54 \mathrm{E}-04$ & $\begin{array}{c}1.16 \mathrm{E}- \\
02\end{array}$ & $\begin{array}{c}5.24 \mathrm{E}- \\
02\end{array}$ & $\begin{array}{l}2.32 \mathrm{E}- \\
02\end{array}$ & 2 & GLS2,GLS \\
\hline 142134 & $\begin{array}{c}\text { asparagine } \\
\text { biosynthesis }\end{array}$ & $4.54 \mathrm{E}-04$ & $\begin{array}{c}1.16 \mathrm{E}- \\
02\end{array}$ & $\begin{array}{c}5.24 \mathrm{E}- \\
02\end{array}$ & $\begin{array}{c}2.32 \mathrm{E}- \\
02\end{array}$ & 2 & ASNS,GOT1 \\
\hline 782395 & $\begin{array}{c}\text { purine } \\
\text { deoxyribonucleosid } \\
\text { es salvage }\end{array}$ & $1.78 \mathrm{E}-03$ & $\begin{array}{c}3.03 \mathrm{E}- \\
02\end{array}$ & $\begin{array}{c}1.37 \mathrm{E}- \\
01\end{array}$ & $\begin{array}{c}9.08 \mathrm{E}- \\
02\end{array}$ & 3 & $\begin{array}{c}\text { NME5,AK5,N } \\
\text { ME1 }\end{array}$ \\
\hline 142344 & $\begin{array}{c}\text { malate-aspartate } \\
\text { shuttle }\end{array}$ & $2.59 E-03$ & $\begin{array}{c}3.31 \mathrm{E}- \\
02\end{array}$ & $\begin{array}{c}1.49 \mathrm{E}- \\
01\end{array}$ & $\begin{array}{c}1.32 \mathrm{E}- \\
01\end{array}$ & 2 & MDH1,GOT1 \\
\hline 782382 & gluconeogenesis & $3.51 \mathrm{E}-03$ & $\begin{array}{c}3.58 \mathrm{E}- \\
02\end{array}$ & $\begin{array}{c}1.62 \mathrm{E}- \\
01\end{array}$ & $\begin{array}{c}1.79 E- \\
01\end{array}$ & 3 & $\begin{array}{c}\text { MDH1,ALDO } \\
\text { A,ENO2 }\end{array}$ \\
\hline 907942 & $\begin{array}{l}\text { superpathway of } \\
\text { purine nucleotide }\end{array}$ & $1.07 \mathrm{E}-02$ & $\begin{array}{c}8.89 \mathrm{E}- \\
02\end{array}$ & $\begin{array}{c}4.02 \mathrm{E}- \\
01\end{array}$ & $\begin{array}{c}5.45 \mathrm{E}- \\
01\end{array}$ & 4 & $\begin{array}{c}\text { HPRT1,NME5 } \\
\text {,AK5,NME1 }\end{array}$ \\
\hline
\end{tabular}


medRxiv preprint doi: https://doi.org/10.1101/2020.12.21.20248688; this version posted December 24, 2020. The copyright holder for this preprint (which was not certified by peer review) is the author/funder, who has granted medRxiv a license to display the preprint in perpetuity. All rights reserved. No reuse allowed without permission.

\begin{tabular}{|c|c|c|c|c|c|c|c|}
\hline 782393 & $\begin{array}{c}\text { CMP } \\
\text { phosphorylation }\end{array}$ & $1.79 \mathrm{E}-02$ & $\begin{array}{c}8.89 \mathrm{E}- \\
02\end{array}$ & $\begin{array}{c}4.02 \mathrm{E}- \\
01\end{array}$ & $\begin{array}{c}9.12 \mathrm{E}- \\
01\end{array}$ & 2 & NME5,NME1 \\
\hline 142346 & $\begin{array}{c}\text { L-cysteine } \\
\text { degradation I }\end{array}$ & $4.18 \mathrm{E}-02$ & $\begin{array}{c}8.89 \mathrm{E}- \\
02\end{array}$ & $\begin{array}{c}4.02 \mathrm{E}- \\
01\end{array}$ & $\begin{array}{c}1.00 \mathrm{E}+0 \\
0\end{array}$ & 1 & GOT1 \\
\hline 545272 & $\begin{array}{c}\text { CMP-N- } \\
\text { acetylneuraminate } \\
\text { biosynthesis I } \\
\text { (eukaryotes) }\end{array}$ & $4.18 \mathrm{E}-02$ & $\begin{array}{c}8.89 \mathrm{E}- \\
02\end{array}$ & $\begin{array}{c}4.02 \mathrm{E}- \\
01\end{array}$ & $\begin{array}{c}1.00 \mathrm{E}+0 \\
0\end{array}$ & 1 & CMAS \\
\hline 545371 & MAP kinase cascade & $4.18 \mathrm{E}-02$ & $\begin{array}{c}8.89 \mathrm{E}- \\
02\end{array}$ & $\begin{array}{c}4.02 \mathrm{E}- \\
01\end{array}$ & $\begin{array}{c}1.00 \mathrm{E}+0 \\
0\end{array}$ & 1 & MAP2K1 \\
\hline
\end{tabular}

KEGG

$377263 \quad$ GABAergic synapse

8.38E-17 1.98E-

$1.20 \mathrm{E}-\quad 1.98 \mathrm{E}-$

21

GNG2,ADC

14

13

14

Y1,SLC12A5

,GABARAPL

1,GABBR2,S

LC32A1,GN

B5,PRKCB,P

RKCG,GAB

RA1,GABRB

3,GABRD, G

ABRG2,GA

D1,GAD2,G

LS2,SLC38A

1, GLS,GNA

O1,GNG3,N

SF

$373900 \quad$ Synaptic vesicle $\quad 8.42 \mathrm{E}-15 \quad 9.94 \mathrm{E}-\quad 6.00 \mathrm{E}-\quad 1.99 \mathrm{E}-\quad 17 \quad$ SLC32A1,AT cycle

$13 \quad 12 \quad 12$

P6V1H,SNA

P25,ATP6V1

A,ATP6V1B

2,ATP6V0C,

ATP6V1E1,

ATP6V1G2,

CPLX2,CPL

X1,STX1A,S

TXBP1,VAM

P2,SYT1,DN

M1,SLC17A 
medRxiv preprint doi: https://doi.org/10.1101/2020.12.21.20248688; this version posted December 24, 2020. The copyright holder for this preprint (which was not certified by peer review) is the author/funder, who has granted medRxiv a license to display the preprint in perpetuity. All rights reserved. No reuse allowed without permission.

\begin{tabular}{|c|c|c|c|c|c|c|c|}
\hline & & & & & & & $6, \mathrm{NSF}$ \\
\hline 537443 & $\begin{array}{c}\text { Retrograde } \\
\text { endocannabinoidsig } \\
\text { naling }\end{array}$ & $2.93 \mathrm{E}-09$ & $\begin{array}{c}2.31 \mathrm{E}- \\
07\end{array}$ & $\begin{array}{l}1.40 \mathrm{E}- \\
06\end{array}$ & $\begin{array}{c}6.93 \mathrm{E}- \\
07\end{array}$ & 15 & $\begin{array}{c}\text { GNG2,ADC } \\
\text { Y1,SLC32A1 } \\
\text {,GNB5,PRK } \\
\text { CB,PRKCG, } \\
\text { MAPK9,GA } \\
\text { BRA1,GABR } \\
\text { B3,GABRD, } \\
\text { GABRG2,IT } \\
\text { PR1,GNAO1, } \\
\text { GNG3,SLC1 } \\
\text { 7A6 }\end{array}$ \\
\hline 552665 & Morphine addiction & $6.24 \mathrm{E}-09$ & $\begin{array}{c}3.68 \mathrm{E}- \\
07\end{array}$ & $\begin{array}{l}2.23 \mathrm{E}- \\
06\end{array}$ & $\begin{array}{l}1.47 \mathrm{E}- \\
06\end{array}$ & 14 & $\begin{array}{c}\text { PDE1A,GNG } \\
\text { 2,ADCY1,G } \\
\text { ABBR2,SLC } \\
\text { 32A1,GNB5, } \\
\text { PRKCB,PRK } \\
\text { CG,GABRA1 } \\
\text {,GABRB3,G } \\
\text { ABRD,GAB } \\
\text { RG2,GNAO1 } \\
\text {,GNG3 }\end{array}$ \\
\hline 952859 & $\begin{array}{l}\text { Oxytocin signaling } \\
\text { pathway }\end{array}$ & $2.34 \mathrm{E}-08$ & $\begin{array}{c}1.11 \mathrm{E}- \\
06\end{array}$ & $\begin{array}{c}6.68 \mathrm{E}- \\
06\end{array}$ & $\begin{array}{l}5.53 \mathrm{E}- \\
06\end{array}$ & 17 & $\begin{array}{c}\text { ADCY1,CAC } \\
\text { NG3,PPP3C } \\
\text { B,PPP3R1,P } \\
\text { RKCB,PRKC } \\
\text { G,MAP2K1,I } \\
\text { TPR1,KCNJ4 } \\
\text {,KCNJ12,GN } \\
\text { AO1,CACNB } \\
\text { 2,CAMK1D, } \\
\text { CALM3,CA } \\
\text { MK2B,CAM } \\
\text { K2G,CAMK } \\
\text { 1G }\end{array}$ \\
\hline 217716 & Cholinergic synapse & $9.46 \mathrm{E}-08$ & $\begin{array}{l}3.72 \mathrm{E}- \\
06\end{array}$ & $\begin{array}{l}2.25 \mathrm{E}- \\
05\end{array}$ & $\begin{array}{l}2.23 \mathrm{E}- \\
05\end{array}$ & 14 & $\begin{array}{l}\text { GNG2,CHR } \\
\text { M1,ADCY1, } \\
\text { GNB5,PRKC } \\
\text { B,PRKCG,M } \\
\text { AP2K1,ITPR } \\
\text { 1,KCNJ4,KC }\end{array}$ \\
\hline
\end{tabular}


medRxiv preprint doi: https://doi.org/10.1101/2020.12.21.20248688; this version posted December 24, 2020. The copyright holder for this preprint (which was not certified by peer review) is the author/funder, who has granted medRxiv a license to display the preprint in perpetuity. All rights reserved. No reuse allowed without permission.

\begin{tabular}{|c|c|c|c|c|c|c|c|}
\hline & & & & & & & $\begin{array}{c}\text { NJ12,GNAO } \\
\text { 1,GNG3,CA } \\
\text { MK2B,CAM } \\
\text { K2G }\end{array}$ \\
\hline 213818 & $\begin{array}{c}\text { Glutamatergic } \\
\text { synapse }\end{array}$ & $1.19 \mathrm{E}-07$ & $\begin{array}{l}4.00 \mathrm{E}- \\
06\end{array}$ & $\begin{array}{l}2.41 \mathrm{E}- \\
05\end{array}$ & $\begin{array}{l}2.80 \mathrm{E}- \\
05\end{array}$ & 14 & $\begin{array}{c}\text { GNG2,ADC } \\
\text { Y1,PPP3CB, } \\
\text { PPP3R1,GN } \\
\text { B5,PRKCB,P } \\
\text { RKCG,GLS2 } \\
\text {,ITPR1,SLC3 } \\
\text { 8A1,GLS,GN } \\
\text { AO1,GNG3, } \\
\text { SLC17A6 }\end{array}$ \\
\hline 83050 & $\begin{array}{c}\text { Calcium signaling } \\
\text { pathway }\end{array}$ & $3.01 \mathrm{E}-07$ & $\begin{array}{c}8.89 \mathrm{E}- \\
06\end{array}$ & $\begin{array}{l}5.37 \mathrm{E}- \\
05\end{array}$ & $\begin{array}{c}7.11 \mathrm{E}- \\
05\end{array}$ & 17 & $\begin{array}{c}\text { PDE1A,CHR } \\
\text { M1,ADCY1, } \\
\text { SLC8A2,PPP } \\
\text { 3CB,PPP3R1 } \\
\text {,PRKCB,PR } \\
\text { KCG,ATP2A } \\
\text { 2,ATP2B1,A } \\
\text { TP2B2,ATP2 } \\
\text { B3,ITPR1,C } \\
\text { ALM3,CAM } \\
\text { K2B,CAMK2 } \\
\text { G,CCKBR }\end{array}$ \\
\hline 1017634 & $\begin{array}{l}\text { cAMPsignaling } \\
\text { pathway }\end{array}$ & $4.60 \mathrm{E}-06$ & $\begin{array}{c}7.23 \mathrm{E}- \\
05\end{array}$ & $\begin{array}{c}4.37 \mathrm{E}- \\
04\end{array}$ & $\begin{array}{c}1.09 \mathrm{E}- \\
03\end{array}$ & 16 & $\begin{array}{c}\text { CHRM1,AD } \\
\text { CY1,GABBR } \\
\text { 2,ATP1A1,A } \\
\text { TP1B1,MAP } \\
\text { K9,MAP2K1, } \\
\text { ATP2A2,AT } \\
\text { P2B1,ATP2B } \\
\text { 2,ATP2B3,N } \\
\text { PY,CALM3, } \\
\text { CAMK2B,C } \\
\text { AMK2G,PA } \\
\text { K1 }\end{array}$ \\
\hline 983748 & $\begin{array}{c}\text { cGMP-PKG } \\
\text { signaling pathway }\end{array}$ & $9.10 \mathrm{E}-06$ & $\begin{array}{c}1.13 \mathrm{E}- \\
04\end{array}$ & $\begin{array}{c}6.83 \mathrm{E}- \\
04\end{array}$ & $\begin{array}{c}2.15 \mathrm{E}- \\
03\end{array}$ & 14 & $\begin{array}{c}\text { ADCY1,SLC } \\
\text { 8A2,PPP3CB } \\
\text {,PPP3R1,PR } \\
\text { KCE,ATP1A }\end{array}$ \\
\hline
\end{tabular}


1,ATP1B1,M

AP2K1,ATP2

A2,ATP2B1,

ATP2B2,AT

P2B3,ITPR1,

CALM3

\begin{tabular}{|c|c|c|c|c|c|c|c|}
\hline \multicolumn{8}{|c|}{ Pathway Interaction Database } \\
\hline 138028 & $\begin{array}{c}\text { Effects of } \\
\text { Botulinum toxin }\end{array}$ & $4.76 \mathrm{E}-07$ & $\begin{array}{l}6.10 \mathrm{E}- \\
05\end{array}$ & $\begin{array}{c}3.31 \mathrm{E}- \\
04\end{array}$ & $\begin{array}{c}6.10 \mathrm{E}- \\
05\end{array}$ & 5 & $\begin{array}{c}\text { SNAP25,ST } \\
\text { X1A,STXBP } \\
\text { 1,VAMP2,S } \\
\text { YT1 }\end{array}$ \\
\hline 137953 & $\begin{array}{c}\text { Role of Calcineurin- } \\
\text { dependent NFAT } \\
\text { signaling in } \\
\text { lymphocytes }\end{array}$ & $1.02 \mathrm{E}-06$ & $\begin{array}{l}6.55 \mathrm{E}- \\
05\end{array}$ & $\begin{array}{l}3.56 \mathrm{E}- \\
04\end{array}$ & $\begin{array}{c}1.31 \mathrm{E}- \\
04\end{array}$ & 9 & $\begin{array}{c}\text { FKBP1A,YW } \\
\text { HAH,YWHA } \\
\text { Z,PRKCE,PR } \\
\text { KCG,MAPK } \\
\text { 9,RAN,CAL } \\
\text { M3,RCAN2 }\end{array}$ \\
\hline 138018 & $\begin{array}{c}\text { Downstream } \\
\text { signaling in naive } \\
\text { CD8+ T cells }\end{array}$ & $7.97 \mathrm{E}-05$ & $\begin{array}{c}3.40 \mathrm{E}- \\
03\end{array}$ & $\begin{array}{c}1.85 \mathrm{E}- \\
02\end{array}$ & $\begin{array}{l}1.02 \mathrm{E}- \\
02\end{array}$ & 7 & $\begin{array}{c}\text { PRKCB,PRK } \\
\text { CE,MAPK9, } \\
\text { MAP2K1,ST } \\
\text { AT4,CALM3 } \\
\text {,EGR1 }\end{array}$ \\
\hline 138057 & $\begin{array}{l}\text { ErbB1 downstream } \\
\text { signaling }\end{array}$ & $3.02 \mathrm{E}-04$ & $\begin{array}{c}9.66 \mathrm{E}- \\
03\end{array}$ & $\begin{array}{l}5.25 \mathrm{E}- \\
02\end{array}$ & $\begin{array}{c}3.86 \mathrm{E}- \\
02\end{array}$ & 9 & $\begin{array}{c}\text { MAP2K4,Y } \\
\text { WHAH,YW } \\
\text { HAZ,CYFIP2 } \\
\text {,MAPK9,MA } \\
\text { P2K1,NCKA } \\
\text { P1,CALM3,E } \\
\text { GR1 }\end{array}$ \\
\hline 169355 & $\begin{array}{l}\text { RAC1 signaling } \\
\text { pathway }\end{array}$ & $9.27 \mathrm{E}-04$ & $\begin{array}{l}2.37 \mathrm{E}- \\
02\end{array}$ & $\begin{array}{c}1.29 \mathrm{E}- \\
01\end{array}$ & $\begin{array}{c}1.19 \mathrm{E}- \\
01\end{array}$ & 6 & $\begin{array}{l}\text { MAP2K4,CY } \\
\text { FIP2,MAPK9 } \\
\text {,NCKAP1,W } \\
\text { ASF1,PAK1 }\end{array}$ \\
\hline 137977 & $\begin{array}{l}\text { Neurotrophic factor- } \\
\text { mediated Trk } \\
\text { receptor signaling }\end{array}$ & $1.13 \mathrm{E}-03$ & $\begin{array}{l}2.37 \mathrm{E}- \\
02\end{array}$ & $\begin{array}{c}1.29 \mathrm{E}- \\
01\end{array}$ & $\begin{array}{c}1.44 \mathrm{E}- \\
01\end{array}$ & 6 & $\begin{array}{c}\text { MAGED1,M } \\
\text { AP2K1,ELM } \\
\text { O1,DNM1,RI } \\
\text { T2,PAK1 }\end{array}$ \\
\hline 169346 & Regulation of RAC1 & $1.30 \mathrm{E}-03$ & $2.37 \mathrm{E}-$ & $1.29 \mathrm{E}-$ & $1.66 \mathrm{E}-$ & 5 & $\begin{array}{l}\text { CHN1,NGEF } \\
\text {,ELMO1,NM }\end{array}$ \\
\hline
\end{tabular}


medRxiv preprint doi: https://doi.org/10.1101/2020.12.21.20248688; this version posted December 24, 2020. The copyright holder for this preprint (which was not certified by peer review) is the author/funder, who has granted medRxiv a license to display the preprint in perpetuity. All rights reserved. No reuse allowed without permission.

\begin{tabular}{|c|c|c|c|c|c|c|c|}
\hline & activity & & 02 & 01 & 01 & & E1,KALRN \\
\hline 138019 & $\begin{array}{l}\text { p75(NTR)-mediated } \\
\text { signaling }\end{array}$ & $1.76 \mathrm{E}-03$ & $\begin{array}{c}2.82 \mathrm{E}- \\
02\end{array}$ & $\begin{array}{c}1.53 \mathrm{E}- \\
01\end{array}$ & $\begin{array}{c}2.26 \mathrm{E}- \\
01\end{array}$ & 6 & $\begin{array}{c}\text { MAGED1,A } \\
\text { PP,BEX3,M } \\
\text { APK9,BEX1, } \\
\text { RTN4 }\end{array}$ \\
\hline 138060 & $\begin{array}{l}\text { Thromboxane A2 } \\
\text { receptor signaling }\end{array}$ & $2.48 \mathrm{E}-03$ & $\begin{array}{l}3.53 \mathrm{E}- \\
02\end{array}$ & $\begin{array}{c}1.92 \mathrm{E}- \\
01\end{array}$ & $\begin{array}{c}3.18 \mathrm{E}- \\
01\end{array}$ & 5 & $\begin{array}{c}\text { GNG2,GNB5 } \\
\text {,PRKCE,PR } \\
\text { KCG,DNM1 }\end{array}$ \\
\hline 138044 & $\begin{array}{c}\text { Trk receptor } \\
\text { signaling mediated } \\
\text { by the MAPK } \\
\text { pathway }\end{array}$ & $3.05 \mathrm{E}-03$ & $\begin{array}{l}3.55 \mathrm{E}- \\
02\end{array}$ & $\begin{array}{c}1.93 \mathrm{E}- \\
01\end{array}$ & $\begin{array}{c}3.90 \mathrm{E}- \\
01\end{array}$ & 4 & $\begin{array}{c}\text { MAP2K1,RI } \\
\text { T2,EGR1,CD } \\
\text { K5 }\end{array}$ \\
\hline
\end{tabular}

REACTOME

\begin{tabular}{|c|c|c|c|c|c|c|c|}
\hline 1268766 & $\begin{array}{l}\text { Transmission across } \\
\text { Chemical Synapses }\end{array}$ & $2.81 \mathrm{E}-23$ & $\begin{array}{l}1.94 \mathrm{E}- \\
20\end{array}$ & $\begin{array}{c}1.38 \mathrm{E}- \\
19\end{array}$ & $\begin{array}{l}1.94 \mathrm{E}- \\
20\end{array}$ & 37 & $\begin{array}{c}\text { NCALD,GN } \\
\text { G2,ADCY1, } \\
\text { CACNG3,G } \\
\text { ABBR2,SLC } \\
\text { 32A1,NPTN, } \\
\text { GNB5,PRKC } \\
\text { B,PRKCG,S } \\
\text { NAP25,GAB } \\
\text { RA1,GABRB } \\
\text { 3,GABRG2, } \\
\text { GAD1,GAD2 } \\
\text {,GLS2,CPLX } \\
\text { 1,SLC38A1, } \\
\text { LIN7B,NEFL } \\
\text {,STX1A,STX } \\
\text { BP1,KCNJ4, } \\
\text { KCNJ12,GLS } \\
\text {,VAMP2,SY } \\
\text { N1,SYN2,SY } \\
\text { T1,GNG3,C } \\
\text { ACNB2,CAL } \\
\text { M3,NSF,CA } \\
\text { MK2B,CAM } \\
\text { K2G,EPB41L } \\
1\end{array}$ \\
\hline 1268763 & Neuronal System & $1.97 \mathrm{E}-19$ & $6.82 \mathrm{E}-$ & $4.85 \mathrm{E}-$ & $1.36 \mathrm{E}-$ & 41 & $\begin{array}{c}\text { NCALD,GN } \\
\text { G2,RTN3,A }\end{array}$ \\
\hline
\end{tabular}


medRxiv preprint doi: https://doi.org/10.1101/2020.12.21.20248688; this version posted December 24, 2020. The copyright holder for this preprint (which was not certified by peer review) is the author/funder, who has granted medRxiv a license to display the preprint in perpetuity. All rights reserved. No reuse allowed without permission.

17

$16 \quad 16$

DCY1,CACN

G3,NRXN1,

GABBR2,SL

C32A1,NPT

N,GNB5,PR

KCB,PRKCG

,SNAP25,GA

BRA1,GABR

B3,GABRG2

,GAD1,GAD

2,GLS2,CPL

X1,LRRTM1

,EPB41L3,SL

C38A1,LIN7

B,NEFL,STX

1A,STXBP1,

$\mathrm{KCNJ} 4, \mathrm{KCN}$

J12,GLS,VA

MP2,SYN1,S

YN2,SYT1,G

NG3,CACNB

2,CALM3,N

SF,CAMK2B

,CAMK2G,E

PB41L1

$1339115 \quad$ Cardiac conduction

1.99E-12

$3.43 \mathrm{E}$

$2.44 \mathrm{E}$

$1.37 \mathrm{E}-$

21

CACNG3,SC

N1B,SCN2B, SCN4B,FGF 12,SLC8A2, KCNIP4,ATP 1A1,ATP1B1 ,ATP2A2,AT P2B1,ATP2B

2,ATP2B3,S

CN3B,ITPR1

,KCNJ4,KCN

J12,CACNB2

,CALM3,CA

MK2B,CAM

$\mathrm{K} 2 \mathrm{G}$

$1268786 \quad$ Neurotransmitter

3.95E-12

$5.46 \mathrm{E}$

3.89E- $2.73 \mathrm{E}-$

21

NCALD, GN

Receptor Binding

10

09

09

G2,ADCY1, CACNG3,G 
medRxiv preprint doi: https://doi.org/10.1101/2020.12.21.20248688; this version posted December 24, 2020. The copyright holder for this preprint (which was not certified by peer review) is the author/funder, who has granted medRxiv a license to display the preprint in perpetuity. All rights reserved. No reuse allowed without permission.

Transmission In The

Postsynaptic Cell
ABBR2,NPT

N,GNB5,PR

KCB,PRKCG

,GABRA1,G

ABRB3,GAB

RG2,NEFL,K

$\mathrm{CNJ} 4, \mathrm{KCNJ} 1$

2,GNG3,CA

LM3,NSF,C

AMK2B,CA

MK2G,EPB4

1L1

1269868

Muscle contraction

3.37E-10

$3.32 \mathrm{E}$

2.37E-

2.33E-

07

08

07

22

TPM1,CACN

G3,SCN1B,S

CN2B,SCN4

B,FGF12,SL

C8A2,KCNIP

4,ATP1A1,A

TP1B1,ATP2

A2,ATP2B1, ATP2B2,AT

P2B3,SCN3B

,ITPR1,KCN

$\mathrm{J} 4, \mathrm{KCNJ} 12, \mathrm{C}$

ACNB2,CAL

M3,CAMK2

B,CAMK2G

1269903

Transmembrane

8.90E-07

3.24E-

$2.30 \mathrm{E}$

$6.15 \mathrm{E}-$

05

04

04
GNG2,ADC

Y1,SLC22A1

7,SLC12A5,S

LC9A6,SLC3

2A1,SLC8A2

,ATP6V1H,G

NB5,PRKAR

1B,ATP1A1,

ATP1B1,AT

P2A2,ATP2B

1,ATP2B2,A

TP2B3, GAB

RA1,GABRB

3,GABRG2,

ATP6V1A,A

TP6V1B2,AT 
medRxiv preprint doi: https://doi.org/10.1101/2020.12.21.20248688; this version posted December 24, 2020. The copyright holder for this preprint (which was not certified by peer review) is the author/funder, who has granted medRxiv a license to display the preprint in perpetuity. All rights reserved. No reuse allowed without permission.

P6V0C,ATP6

V1E1,ATP6

V1G2,STEA

P2,SLC30A3,

SLC38A1,GL

$\mathrm{RB}, \mathrm{GNG} 3, \mathrm{~S}$

LC17A6,CA

LM3,CAMK

2B,CAMK2G

,TUSC3,SLC

6A15

$1269443 \quad$ Signalling by NGF $\quad 5.92 \mathrm{E}-04 \quad 6.02 \mathrm{E}-\quad 4.28 \mathrm{E}-\quad 4.09 \mathrm{E}-$

$03 \quad 02 \quad 01$

22 PDE1A,ADC

Y1,ADCYAP

1,NGEF,FGF

9,MAGED1,

SH3GL2,BE

X3,PRKAR1

B,PRKCE,PR

KCG,MAP2

K1,ITPR1,N

EFL,DNM1,

KALRN,CA

LM3,CAMK

2B,CAMK2G

,RTN4,RIT2,

LINGO1

1269340

Hemostasis

4.60E-03

$3.31 \mathrm{E}$

$2.36 \mathrm{E}-1.00 \mathrm{E}+0$

24

PDE1A,GNG

0

2,FAM3C,A

LDOA,APP,

YWHAZ,SL

C8A2,GNB5,

PRKAR1B,P

RKCB,PRKC

E,PRKCG,A

TP1B1,ATP2

A2,ATP2B1, ATP2B2,AT

P2B3,ITPR1, KIF3C,GNG

3,KLC1,DOC

K3,CALM3, CDK5 
medRxiv preprint doi: https://doi.org/10.1101/2020.12.21.20248688; this version posted December 24, 2020. The copyright holder for this preprint (which was not certified by peer review) is the author/funder, who has granted medRxiv a license to display the preprint in perpetuity. All rights reserved. No reuse allowed without permission.

$1269203 \quad$ Innate Immune

System

\subsection{E-02}

1269543

\begin{abstract}
Signaling by GPCR
\end{abstract}
3.99E-02

$1.77 \mathrm{E}$

01

7.19

$01 \quad 0$

\section{9}

PDE1A,CHG
A,PGAM1,A
DCY1,UBE2
N,FGF9,ALD
OA,MAP2K4

,APP,CYFIP

2,ATP6V1H, PPP3CB,PPP

3R1,PRKAR

1B,PRKCE,P

RKCG,SNAP

25,MAPK9,

MAP2K1,AT

P6V1A,PRSS

3,ATP6V1B2

,ATP6V0C,A

TP6V1E1,AT

P6V1G2,NC

KAP1,ACTR

10,PGRMC1,

ELMO1,ITP

R1,NEFL,PT

PRN2,DNM1

,WASF1,CA

LM3,CAMK

2B,CAMK2G

,SYNGR1,P

AK1

$01+00 \quad 0$
PDE1A,OPN

3, GNG2,CH

RM1,ADCY1

,ADCYAP1,

NGEF,FGF9, $\mathrm{XK}, \mathrm{APP}, \mathrm{GA}$

BBR2,PPP3C

B,PPP3R1,G NB5,PRKAR

1B,PRKCB,P

RKCE,PRKC

G,MAP2K1, REEP1,SST,I TPR1,NEFL, PLPPR4,TA 
C1,GNAO1, GNG3,NPY,

KALRN,FZD

3,CALM3,C

AMK2B,CA

MK2G,RGS4

,RGS7,CCK,

CCKBR,CD

K5

\begin{tabular}{|c|c|c|c|c|c|c|c|}
\hline \multicolumn{8}{|c|}{ Gen MAPP } \\
\hline MAP03070 & $\begin{array}{c}\text { Type III secretion } \\
\text { system }\end{array}$ & 3.69E-05 & $\begin{array}{l}5.00 \mathrm{E}- \\
04\end{array}$ & $\begin{array}{l}2.02 \mathrm{E}- \\
03\end{array}$ & $\begin{array}{c}1.15 \mathrm{E}- \\
03\end{array}$ & 5 & $\begin{array}{c}\text { ATP6V1A,A } \\
\text { TP6V1B2,AT } \\
\text { P6V0C,ATP6 } \\
\text { V1E1,ATP6 } \\
\text { V1G2 }\end{array}$ \\
\hline MAP00193 & ATP synthesis & 3.69E-05 & $\begin{array}{c}5.00 \mathrm{E}- \\
04\end{array}$ & $\begin{array}{c}2.02 \mathrm{E}- \\
03\end{array}$ & $\begin{array}{c}1.15 \mathrm{E}- \\
03\end{array}$ & 5 & $\begin{array}{c}\text { ATP6V1A,A } \\
\text { TP6V1B2,AT } \\
\text { P6V0C,ATP6 } \\
\text { V1E1,ATP6 } \\
\text { V1G2 }\end{array}$ \\
\hline MAP00195 & Photosynthesis & $4.84 \mathrm{E}-05$ & $\begin{array}{c}5.00 \mathrm{E}- \\
04\end{array}$ & $\begin{array}{c}2.02 \mathrm{E}- \\
03\end{array}$ & $\begin{array}{l}1.50 \mathrm{E}- \\
03\end{array}$ & 5 & $\begin{array}{c}\text { ATP6V1A,A } \\
\text { TP6V1B2,AT } \\
\text { P6V0C,ATP6 } \\
\text { V1E1,ATP6 } \\
\text { V1G2 }\end{array}$ \\
\hline MAP00252 & $\begin{array}{l}\text { Alanine and } \\
\text { aspartate } \\
\text { metabolism }\end{array}$ & 8.77E-04 & $\begin{array}{c}6.80 \mathrm{E}- \\
03\end{array}$ & $\begin{array}{l}2.74 \mathrm{E}- \\
02\end{array}$ & $\begin{array}{l}2.72 \mathrm{E}- \\
02\end{array}$ & 4 & $\begin{array}{c}\text { ASNS,GAD1 } \\
\text {,GAD2,GOT } \\
1\end{array}$ \\
\hline MAP00190 & $\begin{array}{c}\text { Oxidative } \\
\text { phosphorylation }\end{array}$ & $2.24 \mathrm{E}-03$ & $\begin{array}{c}1.34 \mathrm{E}- \\
02\end{array}$ & $\begin{array}{l}5.40 \mathrm{E}- \\
02\end{array}$ & $\begin{array}{c}6.96 \mathrm{E}- \\
02\end{array}$ & 5 & $\begin{array}{c}\text { ATP6V1A,A } \\
\text { TP6V1B2,AT } \\
\text { P6V0C,ATP6 } \\
\text { V1E1,ATP6 } \\
\text { V1G2 }\end{array}$ \\
\hline MAP00430 & $\begin{array}{l}\text { Taurine and } \\
\text { hypotaurine } \\
\text { metabolism }\end{array}$ & $2.59 \mathrm{E}-03$ & $\begin{array}{c}1.34 \mathrm{E}- \\
02\end{array}$ & $\begin{array}{l}5.40 \mathrm{E}- \\
02\end{array}$ & $\begin{array}{c}8.04 \mathrm{E}- \\
02\end{array}$ & 2 & GAD1,GAD2 \\
\hline MAP00710 & Carbon fixation & $4.26 \mathrm{E}-03$ & $\begin{array}{c}1.89 \mathrm{E}- \\
02\end{array}$ & $\begin{array}{l}7.59 \mathrm{E}- \\
02\end{array}$ & $\begin{array}{l}1.32 \mathrm{E}- \\
01\end{array}$ & 3 & $\begin{array}{c}\text { MDH1,ALD } \\
\text { OA,GOT1 }\end{array}$ \\
\hline
\end{tabular}


medRxiv preprint doi: https://doi.org/10.1101/2020.12.21.20248688; this version posted December 24, 2020. The copyright holder for this preprint (which was not certified by peer review) is the author/funder, who has granted medRxiv a license to display the preprint in perpetuity. All rights reserved. No reuse allowed without permission.

\begin{tabular}{|c|c|c|c|c|c|c|c|}
\hline MAP00251 & $\begin{array}{l}\text { Glutamate } \\
\text { metabolism }\end{array}$ & $8.14 \mathrm{E}-03$ & $\begin{array}{c}3.16 \mathrm{E}- \\
02\end{array}$ & $\begin{array}{c}1.27 \mathrm{E}- \\
01\end{array}$ & $\begin{array}{c}2.52 \mathrm{E}- \\
01\end{array}$ & 3 & $\begin{array}{c}\text { GAD1,GAD2 } \\
\text {,GOT1 }\end{array}$ \\
\hline MAP00400 & $\begin{array}{c}\text { Phenylalanine } \\
\text { tyrosine and } \\
\text { tryptophan } \\
\text { biosynthesis }\end{array}$ & $1.14 \mathrm{E}-02$ & $\begin{array}{c}3.94 \mathrm{E}- \\
02\end{array}$ & $\begin{array}{c}1.59 \mathrm{E}- \\
01\end{array}$ & $\begin{array}{c}3.55 \mathrm{E}- \\
01\end{array}$ & 2 & GOT1,ENO2 \\
\hline MAP00410 & $\begin{array}{l}\text { beta Alanine } \\
\text { metabolism }\end{array}$ & 4.91E-02 & $\begin{array}{c}1.52 \mathrm{E}- \\
01\end{array}$ & $\begin{array}{c}6.13 \mathrm{E}- \\
01\end{array}$ & $\begin{array}{c}1.00 \mathrm{E}+0 \\
0\end{array}$ & 2 & GAD1,GAD2 \\
\hline
\end{tabular}

MSigDB C2 BIOCARTA (v6.0)

\begin{tabular}{|c|c|c|c|c|c|c|c|}
\hline M15181 & $\begin{array}{l}\text { Regulation of PGC- } \\
1 \mathrm{a}\end{array}$ & $8.21 \mathrm{E}-06$ & $\begin{array}{l}1.31 \mathrm{E}- \\
03\end{array}$ & $\begin{array}{c}7.43 \mathrm{E}- \\
03\end{array}$ & $\begin{array}{c}1.31 \mathrm{E}- \\
03\end{array}$ & 6 & $\begin{array}{c}\text { YWHAH,PP } \\
\text { P3CB,CALM } \\
\text { 3,CAMK2B, } \\
\text { CAMK2G,C } \\
\text { AMK1G }\end{array}$ \\
\hline M13494 & $\begin{array}{c}\text { Bioactive Peptide } \\
\text { Induced Signaling } \\
\text { Pathway }\end{array}$ & 2.92E-05 & $\begin{array}{l}1.97 \mathrm{E}- \\
03\end{array}$ & $\begin{array}{l}1.11 \mathrm{E}- \\
02\end{array}$ & $\begin{array}{c}4.66 \mathrm{E}- \\
03\end{array}$ & 7 & $\begin{array}{c}\text { PRKCB,MA } \\
\text { P2K1,STAT4 } \\
\text {,CALM3,CA } \\
\text { MK2B,CAM } \\
\text { K2G,CDK5 }\end{array}$ \\
\hline M5940 & $\begin{array}{l}\text { Endocytotic role of } \\
\text { NDK, Phosphins } \\
\text { and Dynamin }\end{array}$ & $3.69 \mathrm{E}-05$ & $\begin{array}{l}1.97 \mathrm{E}- \\
03\end{array}$ & $\begin{array}{l}1.11 \mathrm{E}- \\
02\end{array}$ & $\begin{array}{c}5.91 \mathrm{E}- \\
03\end{array}$ & 5 & $\begin{array}{c}\text { AMPH,PPP3 } \\
\text { CB,NME1,D } \\
\text { NM1,CALM } \\
3\end{array}$ \\
\hline M9664 & $\begin{array}{l}\text { Signaling Pathway } \\
\text { from G-Protein } \\
\text { Families }\end{array}$ & $8.14 \mathrm{E}-05$ & $\begin{array}{c}3.26 \mathrm{E}- \\
03\end{array}$ & $\begin{array}{c}1.84 \mathrm{E}- \\
02\end{array}$ & $\begin{array}{l}1.30 \mathrm{E}- \\
02\end{array}$ & 6 & $\begin{array}{c}\text { ADCY1,PPP } \\
\text { 3CB,PRKAR } \\
\text { 1B,PRKCB, } \\
\text { MAP2K1,CA } \\
\text { LM3 }\end{array}$ \\
\hline M3412 & $\begin{array}{l}\mathrm{Ca}++/ \text { Calmodulin- } \\
\text { dependent Protein } \\
\text { Kinase Activation }\end{array}$ & $1.65 \mathrm{E}-04$ & $\begin{array}{c}5.28 \mathrm{E}- \\
03\end{array}$ & $\begin{array}{c}2.98 \mathrm{E}- \\
02\end{array}$ & $\begin{array}{c}2.64 \mathrm{E}- \\
02\end{array}$ & 4 & $\begin{array}{c}\text { CALM3,CA } \\
\text { MK2B,CAM } \\
\text { K2G,CAMK } \\
\text { 1G }\end{array}$ \\
\hline M9070 & $\begin{array}{l}\text { Transcription factor } \\
\text { CREB and its } \\
\text { extracellular signals }\end{array}$ & $2.23 \mathrm{E}-04$ & $\begin{array}{c}5.96 \mathrm{E}- \\
03\end{array}$ & $\begin{array}{c}3.37 \mathrm{E}- \\
02\end{array}$ & $\begin{array}{c}3.57 \mathrm{E}- \\
02\end{array}$ & 5 & $\begin{array}{c}\text { ADCY1,PRK } \\
\text { AR1B,PRKC } \\
\text { B,CAMK2B, } \\
\text { CAMK2G }\end{array}$ \\
\hline
\end{tabular}


medRxiv preprint doi: https://doi.org/10.1101/2020.12.21.20248688; this version posted December 24, 2020. The copyright holder for this preprint (which was not certified by peer review) is the author/funder, who has granted medRxiv a license to display the preprint in perpetuity. All rights reserved. No reuse allowed without permission.

\begin{tabular}{|c|c|c|c|c|c|c|c|}
\hline M7739 & $\begin{array}{c}\text { Links between Pyk2 } \\
\text { and Map Kinases }\end{array}$ & $3.17 \mathrm{E}-04$ & $\begin{array}{c}7.26 \mathrm{E}- \\
03\end{array}$ & $\begin{array}{l}4.10 \mathrm{E}- \\
02\end{array}$ & $\begin{array}{c}5.08 \mathrm{E}- \\
02\end{array}$ & 5 & $\begin{array}{c}\text { MAP2K4,PR } \\
\text { KCB,MAP2 } \\
\text { K1,CALM3, } \\
\text { PAK1 }\end{array}$ \\
\hline M19118 & $\begin{array}{c}\text { Keratinocyte } \\
\text { Differentiation }\end{array}$ & $3.87 \mathrm{E}-04$ & $\begin{array}{c}7.74 \mathrm{E}- \\
03\end{array}$ & $\begin{array}{c}4.38 \mathrm{E}- \\
02\end{array}$ & $\begin{array}{c}6.19 \mathrm{E}- \\
02\end{array}$ & 6 & $\begin{array}{c}\text { ETS2,MAP2 } \\
\text { K4,PRKCB,P } \\
\text { RKCE,PRKC } \\
\text { G,MAP2K1 }\end{array}$ \\
\hline M14899 & $\begin{array}{l}\text { Angiotensin II } \\
\text { mediated activation } \\
\text { of JNK Pathway via } \\
\text { Pyk2 dependent } \\
\text { signaling }\end{array}$ & $6.82 \mathrm{E}-04$ & $\begin{array}{c}1.21 \mathrm{E}- \\
02\end{array}$ & $\begin{array}{c}6.86 \mathrm{E}- \\
02\end{array}$ & $\begin{array}{c}1.09 \mathrm{E}- \\
01\end{array}$ & 5 & $\begin{array}{c}\text { MAP2K4,PR } \\
\text { KCB,MAP2 } \\
\text { K1,CALM3, } \\
\text { PAK1 }\end{array}$ \\
\hline M2288 & $\begin{array}{l}\text { NFAT and } \\
\text { Hypertrophy of the } \\
\text { heart (Transcription } \\
\text { in the broken heart) }\end{array}$ & $9.27 \mathrm{E}-04$ & $\begin{array}{c}1.36 \mathrm{E}- \\
02\end{array}$ & $\begin{array}{c}7.67 \mathrm{E}- \\
02\end{array}$ & $\begin{array}{c}1.48 \mathrm{E}- \\
01\end{array}$ & 6 & $\begin{array}{c}\text { FKBP1A,PPP } \\
\text { 3CB,PRKAR } \\
\text { 1B,MAP2K1, } \\
\text { CALM3,CA } \\
\text { MK1G }\end{array}$ \\
\hline
\end{tabular}

Panther DB

\begin{tabular}{|c|c|c|c|c|c|c|c|}
\hline P00042 & $\begin{array}{c}\text { Muscarinic } \\
\text { acetylcholine } \\
\text { receptor } 1 \text { and } 3 \\
\text { signaling pathway }\end{array}$ & 1.60E-08 & $\begin{array}{c}1.28 \mathrm{E}- \\
06\end{array}$ & $\begin{array}{c}6.34 \mathrm{E}- \\
06\end{array}$ & $\begin{array}{c}1.28 \mathrm{E}- \\
06\end{array}$ & 11 & $\begin{array}{c}\text { CHRM1,GN } \\
\text { B5,PRKCB,P } \\
\text { RKCE,PRKC } \\
\text { G,SNAP25,I } \\
\text { TPR1,STX1 } \\
\text { A,VAMP1,V } \\
\text { AMP2,GNG3 }\end{array}$ \\
\hline P04391 & $\begin{array}{l}\text { Oxytocin receptor } \\
\text { mediated signaling } \\
\text { pathway }\end{array}$ & $1.89 \mathrm{E}-07$ & $\begin{array}{c}6.38 \mathrm{E}- \\
06\end{array}$ & $\begin{array}{c}3.17 \mathrm{E}- \\
05\end{array}$ & $\begin{array}{c}1.51 \mathrm{E}- \\
05\end{array}$ & 10 & $\begin{array}{c}\text { GNG2,GNB5 } \\
\text {,PRKCB,PR } \\
\text { KCE,PRKCG } \\
\text {,SNAP25,VA } \\
\text { MP1,VAMP2 } \\
\text {,GNG3,CAC } \\
\text { NB2 }\end{array}$ \\
\hline P05734 & $\begin{array}{l}\text { Synaptic vesicle } \\
\text { trafficking }\end{array}$ & $3.18 \mathrm{E}-07$ & $\begin{array}{c}6.38 \mathrm{E}- \\
06\end{array}$ & $\begin{array}{c}3.17 \mathrm{E}- \\
05\end{array}$ & $\begin{array}{c}2.55 \mathrm{E}- \\
05\end{array}$ & 7 & $\begin{array}{c}\text { STX1A,STX } \\
\text { BP1,VAMP1, } \\
\text { SYN1,SYP,S } \\
\text { YT1,NSF }\end{array}$ \\
\hline P00040 & $\begin{array}{c}\text { Metabotropic } \\
\text { glutamate receptor }\end{array}$ & $3.27 \mathrm{E}-06$ & $3.30 \mathrm{E}-$ & $1.64 \mathrm{E}-$ & $2.62 \mathrm{E}-$ & 8 & $\begin{array}{l}\text { GNB5,PRKA } \\
\text { R1B,SNAP25 }\end{array}$ \\
\hline
\end{tabular}


medRxiv preprint doi: https://doi.org/10.1101/2020.12.21.20248688; this version posted December 24, 2020. The copyright holder for this preprint (which was not certified by peer review) is the author/funder, who has granted medRxiv a license to display the preprint in perpetuity. All rights reserved. No reuse allowed without permission.

group II pathway

P00003

P00021

P00018

P00018

P00029

Huntington disease

Heterotrimeric Gprotein signaling pathway-Gq alpha and Go alpha mediated pathway

FGF signaling pathway

EGF receptor
ignaling pathway

\begin{abstract}
Alzheimer diseaseamyloid secretase pathway
\end{abstract}

05

$$
04
$$

04

$\begin{array}{ccc}6.65 \mathrm{E}- & 3.30 \mathrm{E}- & 6.65 \mathrm{E}- \\ 05 & 04 & 04\end{array}$

1.18E-04

3.02E-04$$
\text { . }
$$
signaling pathway

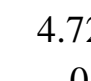

4.7

$2.34 \mathrm{E}$

03

03

$1.01 \mathrm{E}-$ 03

$5.00 \mathrm{E}$

03

$2.42 \mathrm{E}$

02
5.65E-04
$1.74 \mathrm{E}-$ 03
8.63E- 4.52E-
03
02

\begin{abstract}
1.18E-03
\end{abstract}
3.15E-

03
$1.57 \mathrm{E}$

02

02
,STX1A,VA

MP1,VAMP2

,GNAO1,GN

G3

PCSK1,CHR

M1,APP,PR

KCB,PRKCE

,PRKCG,MA

PK9,KLC1,C

ACNB2

GNG2,CHR

M1,PRKCB,

PRKCE,PRK

CG,ITPR1,G

NAO1,GNG3

,RGS4,RGS7

FGF9,FGF12

,MAP2K4,Y

WHAZ,PRK

CB,PRKCE,P

RKCG,MAP

K9,MAP2K1

MAP2K4,Y

WHAH,YW

HAZ,PRKCB

,PRKCE,PR

$\mathrm{KCG}$,

MAPK9,MA

P2K1,STAT4

9 TUBB2A,TU

BB3,MAP2K

4,PACSIN1, CYFIP2,ARF

3,

MAPK9,DY

NC1I1,KAL

RN

P00031
Inflammation mediated by chemokine and
2.02E-02
$3.95 \mathrm{E}$

02

$1.96 \mathrm{E}$

01

.00E

9

GNG2,PRKC

0 PR1,GNG3, 
cytokine signaling

AMK2G,PA

pathway

K5,RGS4,PA

$\mathrm{K} 1$

\begin{tabular}{|c|c|c|c|c|c|c|c|}
\hline \multicolumn{8}{|c|}{ Pathway Ontology } \\
\hline PW:0000027 & glutamate metabolic & $1.20 \mathrm{E}-04$ & $\begin{array}{l}7.90 \mathrm{E}- \\
03\end{array}$ & $\begin{array}{l}3.77 \mathrm{E}- \\
02\end{array}$ & $\begin{array}{l}7.90 \mathrm{E}- \\
03\end{array}$ & 4 & $\begin{array}{c}\text { GAD1,GAD2 } \\
\text {,GLS,GOT1 }\end{array}$ \\
\hline PW:0000674 & $\begin{array}{c}\text { insulin secretion } \\
\text { pathway }\end{array}$ & 4.72E-04 & $\begin{array}{c}1.56 \mathrm{E}- \\
02\end{array}$ & $\begin{array}{c}7.43 \mathrm{E}- \\
02\end{array}$ & $\begin{array}{c}3.11 \mathrm{E}- \\
02\end{array}$ & 4 & $\begin{array}{c}\text { SNAP25,ST } \\
\text { X1A,STXBP } \\
\text { 1,VAMP2 }\end{array}$ \\
\hline PW:0000028 & $\begin{array}{c}\text { alanine and aspartate } \\
\text { metabolic }\end{array}$ & $1.00 \mathrm{E}-03$ & $\begin{array}{l}2.20 \mathrm{E}- \\
02\end{array}$ & $\begin{array}{c}1.05 \mathrm{E}- \\
01\end{array}$ & $\begin{array}{c}6.61 \mathrm{E}- \\
02\end{array}$ & 3 & $\begin{array}{c}\text { GAD1,GAD2 } \\
\text {,GOT1 }\end{array}$ \\
\hline PW:0000057 & carbon fixation & $2.28 \mathrm{E}-03$ & $\begin{array}{c}3.42 \mathrm{E}- \\
02\end{array}$ & $\begin{array}{c}1.63 \mathrm{E}- \\
01\end{array}$ & $\begin{array}{c}1.50 \mathrm{E}- \\
01\end{array}$ & 3 & $\begin{array}{c}\text { MDH1,ALD } \\
\text { OA,GOT1 }\end{array}$ \\
\hline PW:0000274 & $\begin{array}{l}\text { neuron-to-neuron } \\
\text { signaling via the } \\
\text { chemical synapse }\end{array}$ & $2.59 \mathrm{E}-03$ & $\begin{array}{c}3.42 \mathrm{E}- \\
02\end{array}$ & $\begin{array}{c}1.63 \mathrm{E}- \\
01\end{array}$ & $\begin{array}{c}1.71 \mathrm{E}- \\
01\end{array}$ & 2 & $\begin{array}{c}\mathrm{KCNJ} 4, \mathrm{KCN} \\
\mathrm{J} 12\end{array}$ \\
\hline PW:0000018 & Parkinson disease & $4.26 \mathrm{E}-03$ & $\begin{array}{l}3.52 \mathrm{E}- \\
02\end{array}$ & $\begin{array}{c}1.68 \mathrm{E}- \\
01\end{array}$ & $\begin{array}{c}2.81 \mathrm{E}- \\
01\end{array}$ & 3 & $\begin{array}{c}\text { UCHL1,SNC } \\
\text { A,MAPK9 }\end{array}$ \\
\hline PW:0000640 & glycolysis pathway & $9.35 \mathrm{E}-03$ & $\begin{array}{l}5.61 \mathrm{E}- \\
02\end{array}$ & $\begin{array}{c}2.68 \mathrm{E}- \\
01\end{array}$ & $\begin{array}{c}6.17 \mathrm{E}- \\
01\end{array}$ & 3 & $\begin{array}{l}\text { PGAM1,AL } \\
\text { DOA,ENO2 }\end{array}$ \\
\hline PW:0000015 & Alzheimer disease & $1.36 \mathrm{E}-02$ & $\begin{array}{l}7.37 \mathrm{E}- \\
02\end{array}$ & $\begin{array}{c}3.52 \mathrm{E}- \\
01\end{array}$ & $\begin{array}{c}8.97 \mathrm{E}- \\
01\end{array}$ & 3 & $\begin{array}{c}\text { APP,SNCA, } \\
\text { GNAO1 }\end{array}$ \\
\hline PW:0000543 & $\begin{array}{l}\text { protein kinase A } \\
\text { (PKA) signaling }\end{array}$ & $1.71 \mathrm{E}-02$ & $\begin{array}{c}8.04 \mathrm{E}- \\
02\end{array}$ & $\begin{array}{c}3.84 \mathrm{E}- \\
01\end{array}$ & $\begin{array}{c}1.00 \mathrm{E}+0 \\
0\end{array}$ & 4 & $\begin{array}{c}\text { PDE1A,ADC } \\
\text { Y1,AKAP6,P } \\
\text { RKAR1B }\end{array}$ \\
\hline PW:0000448 & $\begin{array}{c}\text { neuropeptide } Y \\
\text { metabolic }\end{array}$ & $2.16 \mathrm{E}-02$ & $\begin{array}{c}9.50 \mathrm{E}- \\
02\end{array}$ & $\begin{array}{c}4.54 \mathrm{E}- \\
01\end{array}$ & $\begin{array}{c}1.00 \mathrm{E}+0 \\
0\end{array}$ & 1 & NPY \\
\hline
\end{tabular}

\section{SMPDB}

\begin{tabular}{|c|c|c|c|c|c|c|c|}
\hline SMP00232 & Cimetidine Pathway & $2.04 \mathrm{E}-05$ & $\begin{array}{c}3.32 \mathrm{E}- \\
04\end{array}$ & $\begin{array}{c}1.78 \mathrm{E}- \\
03\end{array}$ & $\begin{array}{c}2.44 \mathrm{E}- \\
03\end{array}$ & 5 & $\begin{array}{l}\text { SNAP25,SST } \\
\text { „STX1A,VA } \\
\text { MP2,CCKBR }\end{array}$ \\
\hline SMP00129 & $\begin{array}{c}\text { Malate-Aspartate } \\
\text { Shuttle }\end{array}$ & $4.26 \mathrm{E}-03$ & $\begin{array}{c}4.65 \mathrm{E}- \\
02\end{array}$ & $\begin{array}{c}2.50 \mathrm{E}- \\
01\end{array}$ & $\begin{array}{c}5.12 \mathrm{E}- \\
01\end{array}$ & 2 & $\begin{array}{c}\text { MDH1,SLC2 } \\
5 \mathrm{~A} 12\end{array}$ \\
\hline
\end{tabular}


medRxiv preprint doi: https://doi.org/10.1101/2020.12.21.20248688; this version posted December 24, 2020. The copyright holder for this preprint (which was not certified by peer review) is the author/funder, who has granted medRxiv a license to display the preprint in perpetuity.

All rights reserved. No reuse allowed without permission.

\begin{tabular}{|c|c|c|c|c|c|c|c|}
\hline SMP00072 & $\begin{array}{c}\text { Glutamate } \\
\text { Metabolism }\end{array}$ & $5.09 \mathrm{E}-03$ & $\begin{array}{l}5.09 \mathrm{E}- \\
02\end{array}$ & $\begin{array}{c}2.73 \mathrm{E}- \\
01\end{array}$ & $\begin{array}{c}6.11 \mathrm{E}- \\
01\end{array}$ & 3 & $\begin{array}{c}\text { GAD1,GLS2, } \\
\text { GOT1 }\end{array}$ \\
\hline SMP00128 & Gluconeogenesis & 7.03E-03 & $\begin{array}{c}6.17 \mathrm{E}- \\
02\end{array}$ & $\begin{array}{c}3.31 \mathrm{E}- \\
01\end{array}$ & $\begin{array}{c}8.44 \mathrm{E}- \\
01\end{array}$ & 3 & $\begin{array}{l}\text { MDH1,PGA } \\
\text { M1,ALDOA }\end{array}$ \\
\hline SMP00009 & Ammonia Recycling & $1.14 \mathrm{E}-02$ & $\begin{array}{c}6.17 \mathrm{E}- \\
02\end{array}$ & $\begin{array}{c}3.31 \mathrm{E}- \\
01\end{array}$ & $\begin{array}{c}1.00 \mathrm{E}+0 \\
0\end{array}$ & 2 & ASNS,GLS2 \\
\hline SMP00392 & Benzocaine Pathway & $1.79 \mathrm{E}-02$ & $\begin{array}{c}6.17 \mathrm{E}- \\
02\end{array}$ & $\begin{array}{c}3.31 \mathrm{E}- \\
01\end{array}$ & $\begin{array}{c}1.00 \mathrm{E}+0 \\
0\end{array}$ & 2 & $\begin{array}{c}\text { SCN1B,ATP } \\
\text { 1A1 }\end{array}$ \\
\hline SMP00103 & $\begin{array}{c}\text { Cyclothiazide } \\
\text { Pathway }\end{array}$ & $2.16 \mathrm{E}-02$ & $\begin{array}{c}6.17 \mathrm{E}- \\
02\end{array}$ & $\begin{array}{c}3.31 \mathrm{E}- \\
01\end{array}$ & $\begin{array}{c}1.00 \mathrm{E}+0 \\
0\end{array}$ & 2 & $\begin{array}{c}\text { ATP1A1,AT } \\
\text { P1B1 }\end{array}$ \\
\hline SMP00365 & $\begin{array}{c}\text { Gout or Kelley- } \\
\text { Seegmiller } \\
\text { Syndrome }\end{array}$ & $2.16 \mathrm{E}-02$ & $\begin{array}{c}6.17 \mathrm{E}- \\
02\end{array}$ & $\begin{array}{c}3.31 \mathrm{E}- \\
01\end{array}$ & $\begin{array}{c}1.00 \mathrm{E}+0 \\
0\end{array}$ & 1 & HPRT1 \\
\hline SMP00363 & $\begin{array}{c}\text { Ornithine } \\
\text { Aminotransferase } \\
\text { Deficiency (OAT } \\
\text { Deficiency) }\end{array}$ & $2.16 \mathrm{E}-02$ & $\begin{array}{c}6.17 \mathrm{E}- \\
02\end{array}$ & $\begin{array}{c}3.31 \mathrm{E}- \\
01\end{array}$ & $\begin{array}{c}1.00 \mathrm{E}+0 \\
0\end{array}$ & 1 & OAT \\
\hline SMP00375 & Verapamil Pathway & $3.21 \mathrm{E}-02$ & $\begin{array}{c}6.17 \mathrm{E}- \\
02\end{array}$ & $\begin{array}{c}3.31 \mathrm{E}- \\
01\end{array}$ & $\begin{array}{c}1.00 \mathrm{E}+0 \\
0\end{array}$ & 4 & $\begin{array}{c}\text { TPM1,ATP1 } \\
\text { A1,ATP2A2, } \\
\text { KCNJ4 }\end{array}$ \\
\hline
\end{tabular}

Table 3 The enriched pathway terms of the down regulated differentially expressed genes

BIOCYC

\begin{tabular}{cccccccc}
\hline Pathway ID & Pathway Name & P-value & $\begin{array}{c}\text { FDR } \\
\text { B\&H }\end{array}$ & $\begin{array}{c}\text { FDR } \\
\text { B\&Y }\end{array}$ & $\begin{array}{c}\text { Bonferr } \\
\text { oni }\end{array}$ & $\begin{array}{c}\text { Gene } \\
\text { Count }\end{array}$ & Gene \\
\hline 545357 & melatonin & $2.64 \mathrm{E}-$ & $2.54 \mathrm{E}-$ & $1.00 \mathrm{E}+$ & $1.00 \mathrm{E}+0$ & 1 & MAOA \\
& degradation II & 02 & 01 & 00 & 0 & & \\
142377 & proline & $2.64 \mathrm{E}-$ & $2.54 \mathrm{E}-$ & $1.00 \mathrm{E}+$ & $1.00 \mathrm{E}+0$ & 1 & ALDH4A1 \\
& degradation & 02 & 01 & 00 & 0 & & \\
& & & & & & & \\
\hline
\end{tabular}


medRxiv preprint doi: https://doi.org/10.1101/2020.12.21.20248688; this version posted December 24, 2020. The copyright holder for this preprint (which was not certified by peer review) is the author/funder, who has granted medRxiv a license to display the preprint in perpetuity. All rights reserved. No reuse allowed without permission.

\begin{tabular}{|c|c|c|c|c|c|c|c|}
\hline 144181 & Leishmaniasis & $\begin{array}{c}3.61 \mathrm{E}- \\
08\end{array}$ & $\begin{array}{c}8.96 \mathrm{E}- \\
06\end{array}$ & $\begin{array}{l}5.46 \mathrm{E}- \\
05\end{array}$ & $\begin{array}{c}8.96 \mathrm{E}- \\
06\end{array}$ & 13 & $\begin{array}{c}\text { HLA-DMA,HLA- } \\
\text { DMB,HLA- } \\
\text { DOA,HLA- } \\
\text { DPA1,HLA- } \\
\text { DQA1,HLA- } \\
\text { DRA,FCGR1A,IF } \\
\text { NGR1,CYBA,ITG } \\
\text { B1,ITGB2,PTPN6 } \\
\text {,TGFB3 }\end{array}$ \\
\hline 153910 & Phagosome & $\begin{array}{c}8.28 \mathrm{E}- \\
08\end{array}$ & $\begin{array}{c}1.03 \mathrm{E}- \\
05\end{array}$ & $\begin{array}{c}6.26 \mathrm{E}- \\
05\end{array}$ & $\begin{array}{l}2.05 \mathrm{E}- \\
05\end{array}$ & 18 & $\begin{array}{c}\text { HLA-DMA,HLA- } \\
\text { DMB,HLA- } \\
\text { DOA,HLA- } \\
\text { DPA1,HLA- } \\
\text { DQA1,HLA- } \\
\text { DRA,VAMP3,FC } \\
\text { GR1A,SEC61A1, } \\
\text { CYBA,ITGB1,IT } \\
\text { GB2,ITGB5,TAP1 } \\
\text {,ATP6V0E1,LAM } \\
\text { P2,OLR1,CD14 }\end{array}$ \\
\hline 213780 & Tuberculosis & $\begin{array}{c}8.06 \mathrm{E}- \\
07\end{array}$ & $\begin{array}{l}5.00 \mathrm{E}- \\
05\end{array}$ & $\begin{array}{l}3.05 \mathrm{E}- \\
04\end{array}$ & $\begin{array}{c}2.00 \mathrm{E}- \\
04\end{array}$ & 18 & $\begin{array}{c}\text { CEBPB,HLA- } \\
\text { DMA,HLA- } \\
\text { DMB,HLA- } \\
\text { DOA,HLA- } \\
\text { DPA1,HLA- } \\
\text { DQA1,HLA- } \\
\text { DRA,FCER1G,FC } \\
\text { GR1A,IFNGR1,IT } \\
\text { GAX,ITGB2,SYK } \\
\text {,LAMP2,TGFB3, } \\
\text { CD14,CD74,TNF } \\
\text { RSF1A }\end{array}$ \\
\hline 83078 & $\begin{array}{l}\text { Hematopoietic } \\
\text { cell lineage }\end{array}$ & $\begin{array}{c}1.12 \mathrm{E}- \\
06\end{array}$ & $\begin{array}{c}5.56 \mathrm{E}- \\
05\end{array}$ & $\begin{array}{c}3.39 \mathrm{E}- \\
04\end{array}$ & $\begin{array}{c}2.78 \mathrm{E}- \\
04\end{array}$ & 13 & $\begin{array}{c}\text { HLA-DMA,HLA- } \\
\text { DMB,HLA- } \\
\text { DOA,HLA- } \\
\text { DPA1,HLA- } \\
\text { DQA1,HLA- } \\
\text { DRA,FCGR1A,CS } \\
\text { F1R,CSF2RA,CSF } \\
\text { 3R,CD14,CD37,C } \\
\text { D44 }\end{array}$ \\
\hline
\end{tabular}


medRxiv preprint doi: https://doi.org/10.1101/2020.12.21.20248688; this version posted December 24, 2020. The copyright holder for this preprint (which was not certified by peer review) is the author/funder, who has granted medRxiv a license to display the preprint in perpetuity. All rights reserved. No reuse allowed without permission.

\begin{tabular}{|c|c|c|c|c|c|c|c|}
\hline 114228 & $\begin{array}{l}\text { Fc gamma R- } \\
\text { mediated } \\
\text { phagocytosis }\end{array}$ & $\begin{array}{c}3.46 \mathrm{E}- \\
06\end{array}$ & $\begin{array}{c}1.23 \mathrm{E}- \\
04\end{array}$ & $\begin{array}{c}7.48 \mathrm{E}- \\
04\end{array}$ & $\begin{array}{c}8.59 \mathrm{E}- \\
04\end{array}$ & 12 & $\begin{array}{c}\text { FCGR1A,WAS,A } \\
\text { SAP3,SCIN,MYO } \\
\text { 10,INPPL1,SYK, } \\
\text { RAC2,DOCK2,W } \\
\text { ASF2,LYN,HCK }\end{array}$ \\
\hline 169642 & Toxoplasmosis & $\begin{array}{c}6.39 \mathrm{E}- \\
06\end{array}$ & $\begin{array}{c}1.76 \mathrm{E}- \\
04\end{array}$ & $\begin{array}{c}1.07 \mathrm{E}- \\
03\end{array}$ & $\begin{array}{c}1.58 \mathrm{E}- \\
03\end{array}$ & 13 & $\begin{array}{c}\text { HLA-DMA,HLA- } \\
\text { DMB,HLA- } \\
\text { DOA,HLA- } \\
\text { DPA1,HLA- } \\
\text { DQA1,HLA- } \\
\text { DRA,LY96,HSPA } \\
\text { 1A,ALOX5,IFNG } \\
\text { R1,ITGB1,TGFB3 } \\
\text {,TNFRSF1A }\end{array}$ \\
\hline 83122 & $\begin{array}{l}\text { Systemic lupus } \\
\text { erythematosus }\end{array}$ & $\begin{array}{l}3.75 \mathrm{E}- \\
05\end{array}$ & $\begin{array}{c}8.15 \mathrm{E}- \\
04\end{array}$ & $\begin{array}{c}4.96 \mathrm{E}- \\
03\end{array}$ & $\begin{array}{l}9.30 \mathrm{E}- \\
03\end{array}$ & 13 & $\begin{array}{c}\text { HLA-DMA,HLA- } \\
\text { DMB,HLA- } \\
\text { DOA,HLA- } \\
\text { DPA1,HLA- } \\
\text { DQA1,HLA- } \\
\text { DRA,HIST2H2A } \\
\text { A3,FCGR1A,RO6 } \\
\text { 0,C1QA,C1QB,C1 } \\
\text { QC,CD86 }\end{array}$ \\
\hline 83089 & $\begin{array}{l}\text { Regulation of } \\
\text { actin } \\
\text { cytoskeleton }\end{array}$ & $\begin{array}{c}1.17 \mathrm{E}- \\
04\end{array}$ & $\begin{array}{c}1.71 \mathrm{E}- \\
03\end{array}$ & $\begin{array}{c}1.04 \mathrm{E}- \\
02\end{array}$ & $\begin{array}{l}2.91 \mathrm{E}- \\
02\end{array}$ & 16 & $\begin{array}{c}\text { FGFR3,ARHGEF } \\
\text { 6,EZR,WAS,MSN } \\
\text {,MYL12A,SCIN,I } \\
\text { TGAX,ITGB1,IT } \\
\text { GB2,ITGB4,ITGB } \\
\text { 5,GNA12,RAC2,C } \\
\text { D14,WASF2 }\end{array}$ \\
\hline 373901 & $\begin{array}{l}\text { HTLV-I } \\
\text { infection }\end{array}$ & $\begin{array}{c}2.58 \mathrm{E}- \\
03\end{array}$ & $\begin{array}{c}2.55 \mathrm{E}- \\
02\end{array}$ & $\begin{array}{c}1.55 \mathrm{E}- \\
01\end{array}$ & $\begin{array}{c}6.40 \mathrm{E}- \\
01\end{array}$ & 15 & $\begin{array}{c}\text { CDKN1A,HLA- } \\
\text { DMA,HLA- } \\
\text { DMB,HLA- } \\
\text { DOA,HLA- } \\
\text { DPA1,HLA- } \\
\text { DQA1,HLA- } \\
\text { DRA,FZD9,MSX1 } \\
\text {,CCND1,ITGB2,S } \\
\text { TAT5A,TSPO,TG } \\
\text { FB3,TNFRSF1A }\end{array}$ \\
\hline
\end{tabular}


medRxiv preprint doi: https://doi.org/10.1101/2020.12.21.20248688; this version posted December 24, 2020. The copyright holder for this preprint (which was not certified by peer review) is the author/funder, who has granted medRxiv a license to display the preprint in perpetuity. All rights reserved. No reuse allowed without permission.

$782000 \quad$ Proteoglycans in

$6.27 \mathrm{E}$

$4.51 \mathrm{E}$

$2.75 \mathrm{E}$

$1.00 \mathrm{E}+0$

12

CDKN1A,FZD9,S cancer 03

02

01

0

DC4,EZR,MSN,C

CND1,ITGB1,ITG

B5,PDCD4,PTPN

6,CD44,HCLS1

\section{Pathway Interaction Database}

\begin{tabular}{|c|c|c|c|c|c|c|c|}
\hline 169353 & $\begin{array}{l}\text { Validated } \\
\text { targets of C- } \\
\text { MYC } \\
\text { transcriptional } \\
\text { repression }\end{array}$ & $\begin{array}{l}3.62 \mathrm{E}- \\
07\end{array}$ & $\begin{array}{l}4.56 \mathrm{E}- \\
05\end{array}$ & $\begin{array}{l}2.47 \mathrm{E}- \\
04\end{array}$ & $\begin{array}{c}4.56 \mathrm{E}- \\
05\end{array}$ & 11 & $\begin{array}{c}\text { CDKN1A,CEBPA } \\
\text {,CEBPD,NDRG2, } \\
\text { NDRG1,IRF8,SL } \\
\text { C11A1,CCND1,IT } \\
\text { GB1,ITGB4,HDA } \\
\text { C1 }\end{array}$ \\
\hline 137910 & $\begin{array}{c}\text { CXCR4- } \\
\text { mediated } \\
\text { signaling events }\end{array}$ & $\begin{array}{c}1.37 \mathrm{E}- \\
04\end{array}$ & $\begin{array}{c}8.64 \mathrm{E}- \\
03\end{array}$ & $\begin{array}{l}4.68 \mathrm{E}- \\
02\end{array}$ & $\begin{array}{c}1.73 \mathrm{E}- \\
02\end{array}$ & 9 & $\begin{array}{c}\text { HLA- } \\
\text { DRA,FGR,CSK,I } \\
\text { TGB1,STAT5A,P } \\
\text { TPN6,RGS1,LYN, } \\
\text { HCK }\end{array}$ \\
\hline 138022 & $\begin{array}{c}\text { Class I PI3K } \\
\text { signaling events }\end{array}$ & $\begin{array}{l}5.79 \mathrm{E}- \\
04\end{array}$ & $\begin{array}{c}2.43 \mathrm{E}- \\
02\end{array}$ & $\begin{array}{c}1.32 \mathrm{E}- \\
01\end{array}$ & $\begin{array}{l}7.30 \mathrm{E}- \\
02\end{array}$ & 6 & $\begin{array}{c}\text { FGR,ARHGEF6,S } \\
\text { GK1,INPPL1,LY } \\
\text { N,HCK }\end{array}$ \\
\hline 138017 & $\begin{array}{l}\text { Signaling events } \\
\text { mediated by } \\
\text { PTP1B }\end{array}$ & $\begin{array}{c}1.69 \mathrm{E}- \\
03\end{array}$ & $\begin{array}{l}5.28 \mathrm{E}- \\
02\end{array}$ & $\begin{array}{c}2.86 \mathrm{E}- \\
01\end{array}$ & $\begin{array}{c}2.13 \mathrm{E}- \\
01\end{array}$ & 6 & $\begin{array}{c}\text { FGR,CSF1R,CSK, } \\
\text { STAT5A,LYN,HC } \\
\text { K }\end{array}$ \\
\hline 137939 & $\begin{array}{l}\text { Direct p53 } \\
\text { effectors }\end{array}$ & $\begin{array}{l}2.14 \mathrm{E}- \\
03\end{array}$ & $\begin{array}{l}5.28 \mathrm{E}- \\
02\end{array}$ & $\begin{array}{c}2.86 \mathrm{E}- \\
01\end{array}$ & $\begin{array}{c}2.70 \mathrm{E}- \\
01\end{array}$ & 10 & $\begin{array}{c}\text { CDKN1A,NDRG1 } \\
\text {,DDIT4,RGCC,PY } \\
\text { CARD,SPP1,BCL } \\
\text { 6,TAP1,GPX1,TP } \\
\text { 53BP2 }\end{array}$ \\
\hline 138071 & $\begin{array}{l}\text { PDGFR-beta } \\
\text { signaling } \\
\text { pathway }\end{array}$ & $\begin{array}{l}2.52 \mathrm{E}- \\
03\end{array}$ & $\begin{array}{l}5.28 \mathrm{E}- \\
02\end{array}$ & $\begin{array}{c}2.86 \mathrm{E}- \\
01\end{array}$ & $\begin{array}{c}3.17 \mathrm{E}- \\
01\end{array}$ & 6 & $\begin{array}{c}\text { SLC9A3R1,FGR, } \\
\text { CSK,STAT5A,LY } \\
\text { N,HCK }\end{array}$ \\
\hline 138073 & $\begin{array}{c}\text { C-MYB } \\
\text { transcription } \\
\text { factor network }\end{array}$ & $\begin{array}{c}3.85 \mathrm{E}- \\
03\end{array}$ & $\begin{array}{c}6.45 \mathrm{E}- \\
02\end{array}$ & $\begin{array}{c}3.49 \mathrm{E}- \\
01\end{array}$ & $\begin{array}{c}4.85 \mathrm{E}- \\
01\end{array}$ & 7 & $\begin{array}{l}\text { CDKN1A,CEBPA } \\
\text {,CEBPB,CEBPD, } \\
\text { HIPK2,CSF1R,CC } \\
\text { ND1 }\end{array}$ \\
\hline 138060 & $\begin{array}{l}\text { Thromboxane } \\
\text { A2 receptor }\end{array}$ & $\begin{array}{l}5.72 \mathrm{E}- \\
03\end{array}$ & $\begin{array}{l}8.01 \mathrm{E}- \\
02\end{array}$ & $\begin{array}{c}4.34 \mathrm{E}- \\
01\end{array}$ & $\begin{array}{c}7.21 \mathrm{E}- \\
01\end{array}$ & 5 & $\begin{array}{l}\text { SLC9A3R1,FGR, } \\
\text { SYK,LYN,HCK }\end{array}$ \\
\hline
\end{tabular}


medRxiv preprint doi: https://doi.org/10.1101/2020.12.21.20248688; this version posted December 24, 2020. The copyright holder for this preprint (which was not certified by peer review) is the author/funder, who has granted medRxiv a license to display the preprint in perpetuity. All rights reserved. No reuse allowed without permission.

signaling

\begin{tabular}{cccccccc}
138050 & $\begin{array}{c}\text { Fc-epsilon } \\
\text { receptor I }\end{array}$ & $1.76 \mathrm{E}-$ & $1.69 \mathrm{E}-$ & $9.14 \mathrm{E}-$ & $1.00 \mathrm{E}+0$ & 5 & FCER1G,LAT2,S \\
& 02 & 01 & 01 & 0 & YK,LYN,HCLS1 \\
signaling in & & & & & \\
mast cells & & & & & \\
\multirow{2}{*}{137998} & TCR signaling & $1.88 \mathrm{E}-$ & $1.69 \mathrm{E}-$ & $9.14 \mathrm{E}-$ & $1.00 \mathrm{E}+0$ & HLA- \\
& in naive CD4+ T & 02 & 01 & 01 & 0 & DRA,WAS,CSK,P \\
& cells & & & & & TPN6,CD86
\end{tabular}

REACTOME

\begin{tabular}{|c|c|c|c|c|c|c|c|}
\hline 1457780 & $\begin{array}{c}\text { Neutrophil } \\
\text { degranulation }\end{array}$ & $\begin{array}{c}1.19 \mathrm{E}- \\
09\end{array}$ & $\begin{array}{c}8.57 \mathrm{E}- \\
07\end{array}$ & $\begin{array}{c}6.20 \mathrm{E}- \\
06\end{array}$ & $\begin{array}{c}9.26 \mathrm{E}- \\
07\end{array}$ & 38 & $\begin{array}{c}\text { SERPINA3,ATP8 } \\
\text { B4,CLEC5A,CD3 } \\
\text { 00A,CTSC,TMC6, } \\
\text { TYROBP,SERPIN } \\
\text { B6,FCER1G,FGR, } \\
\text { HSPA1A,ALOX5, } \\
\text { NPC2,PRDX6,SL } \\
\text { C2A5,RHOG,SLC } \\
\text { 11A1,PRCP,PYC } \\
\text { ARD,RNASET2, } \\
\text { VAMP8,CTSH,C } \\
\text { YBA,ITGAX,AD } \\
\text { A2,ITGB2,TMBI } \\
\text { M1,PTPN6,BST2, } \\
\text { DOCK2,LILRB3, } \\
\text { RAB31,LAMP2,O } \\
\text { LR1,CD14,CD44, } \\
\text { CD68,STOM }\end{array}$ \\
\hline 1269310 & $\begin{array}{c}\text { Cytokine } \\
\text { Signaling in } \\
\text { Immune system }\end{array}$ & $\begin{array}{c}2.19 \mathrm{E}- \\
09\end{array}$ & $\begin{array}{c}8.57 \mathrm{E}- \\
07\end{array}$ & $\begin{array}{c}6.20 \mathrm{E}- \\
06\end{array}$ & $\begin{array}{c}1.71 \mathrm{E}- \\
06\end{array}$ & 49 & $\begin{array}{c}\text { CDKN1A,CEBPD } \\
\text {,MAOA,HLA- } \\
\text { DPA1,HLA- } \\
\text { DQA1,HLA- } \\
\text { DRA,HMOX1,FC } \\
\text { GR1A,IFITM3,FG } \\
\text { FR3,ALOX5,IL17 } \\
\text { RB,APBB1IP,IRF } \\
\text { 8,IFITM2,IFNGR } \\
\text { 1,MT2A,CSF1R,C } \\
\text { SF2RA,CSF3R,CS } \\
\text { K,XAF1,IL13RA1 } \\
\text {,TNFSF14,INPPL } \\
\text { 1,SPTBN1,GBP2, }\end{array}$ \\
\hline
\end{tabular}


medRxiv preprint doi: https://doi.org/10.1101/2020.12.21.20248688; this version posted December 24, 2020. The copyright holder for this preprint (which was not certified by peer review) is the author/funder, who has granted medRxiv a license to display the preprint in perpetuity. All rights reserved. No reuse allowed without permission.

CCND1,BCL6,IT

GAX,ITGB1,ITG

B2,STAT5A,PTP

N6,BST2,SYK,A

DAM17,OAS2,G

RIN2C,LGALS9,

HAVCR2,EBI3,TI

MP1,CD86,CD44,

TNFRSF1A,LYN, HCK,HERC5

1269340 Hemostasis $1.92 \mathrm{E}-\quad 5.00 \mathrm{E}-\quad 3.62 \mathrm{E}-\quad 1.50 \mathrm{E}-$ 08

06

05

05

42

SERPINA3,PDE9

A,HMG20B,SERP

INB6,FCER1G,R

APGEF3,FGR,ME

RTK,PLEK,SDC4

,APBB1IP,VEGF

B,PDPN,RHOG,C

SF2RA,CSK,PRC

P,ATP1B2,ITGA

X,ITGB1,ITGB2,

PTPN6,SYK,KCN

MA1,GNA12,RA

C2,FERMT3,DOC

K2,RARRES2,LA

MP2,SLC7A7,OL

R1,TGFB3,SLC16

A3,TIMP1,SYTL4

,GYPC,CD44,LH

FPL2,CD74,LYN, HDAC1

$1269203 \quad$ Innate Immune $\quad 5.54 \mathrm{E}-\quad 1.08 \mathrm{E}-\quad 7.84 \mathrm{E}-\quad 4.33 \mathrm{E}-\quad 66$ System

08

05

05

05
CDKN1A,SERPI

NA3,ATP8B4,CL

EC5A,CD300A,C

TSC,TMC6,LY96, S100A1,TYROBP

,SERPINB6,FCER

1G,FCGR1A,FOX

O4,FGFR3,FGR,H

SPA1A,LY86,AL

OX5,APBB1IP,W

AS,LAT2,NPC2,P

RDX6,SLC2A5,R

HOG,SLC11A1,C 
medRxiv preprint doi: https://doi.org/10.1101/2020.12.21.20248688; this version posted December 24, 2020. The copyright holder for this preprint (which was not certified by peer review) is the author/funder, who has granted medRxiv a license to display the preprint in perpetuity. All rights reserved. No reuse allowed without permission.

1270244

1269350

1269373
Extracellular matrix organization

\begin{abstract}
Platelet
\end{abstract} activation, signaling and aggregation
$2.66 \mathrm{E}-\quad 3.84 \mathrm{E}-\quad 2.78 \mathrm{E}-\quad 2.08 \mathrm{E}-$ 06 04

03

03
SF2RA,CSK,PRC P,PYCARD,RNA SET2,VAMP8,CT SH,CYBA,MYO1 0,SPTBN1,ITGA X,ADA2,ITGB2,T MBIM1,PTPN6,B ST2,MAPKAPK3, SYK,C1QA,C1QB ,C1QC,UNC93B1, DOCK2,LILRB3, RAB31,ATP6V0E 1,LAMP2,GRIN2 C,OLR1,CD14,C D86,WASF2,TLR 5,CD44,CD68,LY N,HCK,HERC5,S TOM 23

FBLN1,PLOD1,S DC4,COL20A1,B CAN,NCAN,HTR A1,SPP1,ITGAX,I TGB1,ITGB2,ITG B4,ITGB5,BGN,A DAM17,DDR1,PL OD3,COLGALT1, TGFB3,TIMP1,C D44,LRP4,CD151

$\begin{array}{ccccc}3.70 \mathrm{E}- & 3.84 \mathrm{E}- & 2.78 \mathrm{E}- & 2.90 \mathrm{E}- & 22 \\ 06 & 04 & 03 & 03 & \end{array}$

SERPINA3,FCER 1G,RAPGEF3,PL EK,APBB1IP,VE GFB,PDPN,RHO G,CSF2RA,CSK,P TPN6,SYK,GNA1 2,RAC2,FERMT3, RARRES2,LAMP 2,TGFB3,TIMP1, SYTL4,LHFPL2,L $\mathrm{YN}$

$\begin{array}{ccccc}\text { Cell surface } & 3.99 \mathrm{E}- & 3.84 \mathrm{E}- & 2.78 \mathrm{E}- & 3.12 \mathrm{E}- \\ \text { interactions at } & 06 & 04 & 03 & 03\end{array}$

15
FCER1G,MERTK ,SDC4,ATP1B2,I TGAX,ITGB1,IT 
medRxiv preprint doi: https://doi.org/10.1101/2020.12.21.20248688; this version posted December 24, 2020. The copyright holder for this preprint (which was not certified by peer review) is the author/funder, who has granted medRxiv a license to display the preprint in perpetuity. All rights reserved. No reuse allowed without permission.

the vascular wall

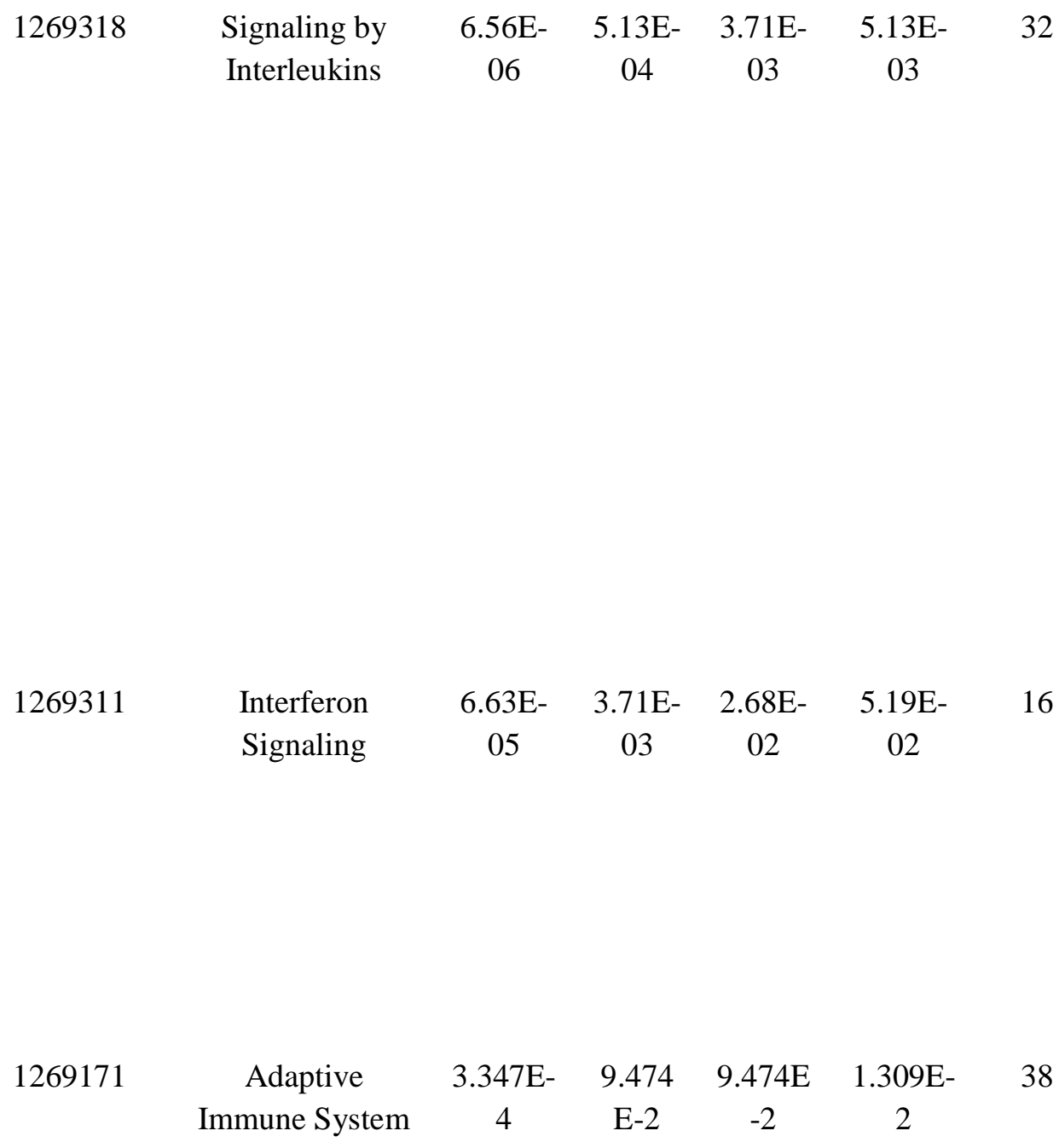

GB2,PTPN6,SLC7

A7,OLR1,SLC16

A3,GYPC,CD44, CD74,LYN

CDKN1A,CEBPD ,MAOA,HMOX1, FGFR3,ALOX5,I

L17RB,APBB1IP, CSF1R,CSF2RA, CSF3R,CSK,IL13 RA1,INPPL1,SPT BN1,CCND1,BCL 6,ITGAX,ITGB1,I TGB2,STAT5A,P TPN6,SYK,GRIN 2C,LGALS9,HAV CR2,EBI3,TIMP1, CD86,TNFRSF1A ,LYN,HCK

HLA-DPA1,HLADQA1,HLA-

DRA,FCGR1A,IFI TM3,IRF8,IFITM 2,IFNGR1,MT2A, XAF1,GBP2,PTP N6,BST2,OAS2,C D44,HERC5

CDKN1A,HLA-
DMA,HLA-
DMB,HLA-
DOA,HLA-
DPA1,HLA-
DQA1,CD300A,H
LA-
DRA,CTSC,LY96
,VAMP3,TYROB
P,FCGR1A,RAPG
EF3,FOXO4,FGF
R3,SEC61A1,WA
S,SIPA1,CSK,VA
MP8,CTSH,CYB
A,ITGB1,ITGB2,


LILRB1,ITGB5,H

CST,PTPN6,SIGL

EC10,SYK,TAP1,

LILRB3,CD14,CD

86,CD74,LYN,HE

RC5

\begin{tabular}{lccccccc}
\hline \multicolumn{7}{c}{ Gen MAPP } \\
\hline MAP00562 & Inositol & $1.20 \mathrm{E}-$ & $3.24 \mathrm{E}-$ & $1.00 \mathrm{E}+$ & $3.24 \mathrm{E}-$ & 3 & PLCD1, \\
& $\begin{array}{c}\text { phosphate } \\
\text { metabolism }\end{array}$ & 02 & 01 & 00 & 01 & TPKB \\
& & & & & & \\
\hline
\end{tabular}

\section{MSigDB C2 BIOCARTA (v6.0)}

\begin{tabular}{|c|c|c|c|c|c|c|c|}
\hline M4956 & $\begin{array}{l}\text { Monocyte and } \\
\text { its Surface } \\
\text { Molecules }\end{array}$ & $\begin{array}{l}2.38 \mathrm{E}- \\
03\end{array}$ & $\begin{array}{l}1.65 \mathrm{E}- \\
01\end{array}$ & $\begin{array}{l}9.25 \mathrm{E}- \\
01\end{array}$ & $\begin{array}{c}3.64 \mathrm{E}- \\
01\end{array}$ & 3 & $\begin{array}{c}\text { ITGB1,ITGB2,CD } \\
44\end{array}$ \\
\hline M12399 & $\begin{array}{c}\text { Cystic Fibrosis } \\
\text { Transmembrane } \\
\text { Conductance } \\
\text { Regulator And } \\
\text { Beta } 2 \\
\text { Adrenergic } \\
\text { Receptor } \\
\text { Pathway }\end{array}$ & $\begin{array}{c}3.11 \mathrm{E}- \\
03\end{array}$ & $\begin{array}{c}1.65 \mathrm{E}- \\
01\end{array}$ & $\begin{array}{c}9.25 \mathrm{E}- \\
01\end{array}$ & $\begin{array}{c}4.77 \mathrm{E}- \\
01\end{array}$ & 3 & $\begin{array}{c}\text { SLC9A3R1,ADR } \\
\text { B2,EZR }\end{array}$ \\
\hline M11106 & $\begin{array}{c}\text { Inactivation of } \\
\text { Gsk3 by AKT } \\
\text { causes } \\
\text { accumulation of } \\
\text { b-catenin in } \\
\text { Alveolar } \\
\text { Macrophages }\end{array}$ & $\begin{array}{c}4.71 \mathrm{E}- \\
03\end{array}$ & $\begin{array}{c}1.65 \mathrm{E}- \\
01\end{array}$ & $\begin{array}{l}9.25 \mathrm{E}- \\
01\end{array}$ & $\begin{array}{c}7.21 \mathrm{E}- \\
01\end{array}$ & 4 & $\begin{array}{c}\text { LY96,CCND1,GJ } \\
\text { A1,CD14 }\end{array}$ \\
\hline M7146 & $\begin{array}{c}\text { Classical } \\
\text { Complement } \\
\text { Pathway }\end{array}$ & $\begin{array}{c}4.96 \mathrm{E}- \\
03\end{array}$ & $\begin{array}{c}1.65 \mathrm{E}- \\
01\end{array}$ & $\begin{array}{c}9.25 \mathrm{E}- \\
01\end{array}$ & $\begin{array}{c}7.59 \mathrm{E}- \\
01\end{array}$ & 3 & $\begin{array}{c}\mathrm{C} 1 \mathrm{Q} A, \mathrm{C} 1 \mathrm{QB}, \mathrm{C} 1 \mathrm{Q} \\
\mathrm{C}\end{array}$ \\
\hline M648 & $\begin{array}{c}\text { Cell Cycle: } \\
\text { G1/S Check } \\
\text { Point }\end{array}$ & $\begin{array}{c}5.39 \mathrm{E}- \\
03\end{array}$ & $\begin{array}{c}1.65 \mathrm{E}- \\
01\end{array}$ & $\begin{array}{c}9.25 \mathrm{E}- \\
01\end{array}$ & $\begin{array}{c}8.24 \mathrm{E}- \\
01\end{array}$ & 4 & $\begin{array}{l}\text { CDKN1A,CCND1 } \\
\text {,TGFB3,HDAC1 }\end{array}$ \\
\hline M917 & $\begin{array}{c}\text { Complement } \\
\text { Pathway }\end{array}$ & $\begin{array}{l}1.20 \mathrm{E}- \\
02\end{array}$ & $\begin{array}{c}2.30 \mathrm{E}- \\
01\end{array}$ & $\begin{array}{c}1.00 \mathrm{E}+ \\
00\end{array}$ & $\begin{array}{c}1.00 \mathrm{E}+0 \\
0\end{array}$ & 3 & $\begin{array}{c}\mathrm{C} 1 \mathrm{QA}, \mathrm{C} 1 \mathrm{QB}, \mathrm{C} 1 \mathrm{Q} \\
\mathrm{C}\end{array}$ \\
\hline
\end{tabular}


medRxiv preprint doi: https://doi.org/10.1101/2020.12.21.20248688; this version posted December 24, 2020. The copyright holder for this preprint (which was not certified by peer review) is the author/funder, who has granted medRxiv a license to display the preprint in perpetuity. All rights reserved. No reuse allowed without permission.

\begin{tabular}{|c|c|c|c|c|c|c|c|}
\hline M6705 & $\begin{array}{c}\text { Th1/Th2 } \\
\text { Differentiation }\end{array}$ & $\begin{array}{l}1.20 \mathrm{E}- \\
02\end{array}$ & $\begin{array}{c}2.30 \mathrm{E}- \\
01\end{array}$ & $\begin{array}{c}1.00 \mathrm{E}+ \\
00\end{array}$ & $\begin{array}{c}1.00 \mathrm{E}+0 \\
0\end{array}$ & 3 & $\begin{array}{c}\text { HLA- } \\
\text { DRA,IFNGR1,CD } \\
86\end{array}$ \\
\hline M9546 & $\begin{array}{l}\text { Chaperones } \\
\text { modulate } \\
\text { interferon } \\
\text { Signaling } \\
\text { Pathway }\end{array}$ & $\begin{array}{l}1.20 \mathrm{E}- \\
02\end{array}$ & $\begin{array}{c}2.30 \mathrm{E}- \\
01\end{array}$ & $\begin{array}{c}1.00 \mathrm{E}+ \\
00\end{array}$ & $\begin{array}{c}1.00 \mathrm{E}+0 \\
0\end{array}$ & 3 & $\begin{array}{c}\text { HSPA1A,IFNGR1 } \\
\text {,TNFRSF1A }\end{array}$ \\
\hline M5880 & $\begin{array}{l}\text { Genes encoding } \\
\text { proteins } \\
\text { affiliated } \\
\text { structurally or } \\
\text { functionally to } \\
\text { extracellular } \\
\text { matrix proteins }\end{array}$ & $\begin{array}{c}3.26 \mathrm{E}- \\
02\end{array}$ & $\begin{array}{c}3.12 \mathrm{E}- \\
01\end{array}$ & $\begin{array}{c}1.00 \mathrm{E}+ \\
00\end{array}$ & $\begin{array}{c}1.00 \mathrm{E}+0 \\
0\end{array}$ & 9 & $\begin{array}{l}\text { CLEC5A,GPC5,S } \\
\text { DC4,PLXNB1,C1 } \\
\text { QA,C1QB,C1QC, } \\
\text { LGALS9,PLXDC } \\
2\end{array}$ \\
\hline M5885 & $\begin{array}{c}\text { Ensemble of } \\
\text { genes encoding } \\
\text { ECM-associated } \\
\text { proteins } \\
\text { including ECM- } \\
\text { affilaited } \\
\text { proteins, ECM } \\
\text { regulators and } \\
\text { secreted factors }\end{array}$ & $\begin{array}{c}4.99 \mathrm{E}- \\
02\end{array}$ & $\begin{array}{c}3.54 \mathrm{E}- \\
01\end{array}$ & $\begin{array}{c}1.00 \mathrm{E}+ \\
00\end{array}$ & $\begin{array}{c}1.00 \mathrm{E}+0 \\
0\end{array}$ & 27 & $\begin{array}{c}\text { SERPINA3,CLEC } \\
\text { 5A,CTSC,S100A1 } \\
\text {,SERPINB6,GPC5 } \\
\text {,PLOD1,SDC4,PL } \\
\text { XNB1,VEGFB,S1 } \\
\text { 00A16,PAMR1,C } \\
\text { TSH,MEGF10,HT } \\
\text { RA1,TNFSF14,C1 } \\
\text { QA,C1QB,C1QC, } \\
\text { ADAM17,PLOD3, } \\
\text { LGALS9,TGFB3, } \\
\text { PLXDC2,EBI3,TI } \\
\text { MP1,ANGPTL4 }\end{array}$ \\
\hline \multicolumn{8}{|c|}{ Panther DB } \\
\hline P00053 & $\mathrm{T}$ cell activation & $\begin{array}{c}7.21 \mathrm{E}- \\
04\end{array}$ & $\begin{array}{c}3.40 \mathrm{E}- \\
02\end{array}$ & $\begin{array}{c}1.64 \mathrm{E}- \\
01\end{array}$ & $\begin{array}{c}4.97 \mathrm{E}- \\
02\end{array}$ & 8 & $\begin{array}{c}\text { HLA-DOA,HLA- } \\
\text { DPA1,HLA- } \\
\text { DQA1,HLA- } \\
\text { DRA,WAS,CSK, } \\
\text { CD86,CD74 }\end{array}$ \\
\hline P04372 & $\begin{array}{l}5- \\
\text { Hydroxytryptam } \\
\text { ine degredation }\end{array}$ & $\begin{array}{c}9.85 \mathrm{E}- \\
04\end{array}$ & $\begin{array}{c}3.40 \mathrm{E}- \\
02\end{array}$ & $\begin{array}{c}1.64 \mathrm{E}- \\
01\end{array}$ & $\begin{array}{c}6.80 \mathrm{E}- \\
02\end{array}$ & 4 & $\begin{array}{c}\text { MAOA,ALDH4A } \\
\text { 1,ALDH7A1,ALD } \\
\text { H1L1 }\end{array}$ \\
\hline P02776 & $\begin{array}{l}\text { Serine glycine } \\
\text { biosynthesis }\end{array}$ & $\begin{array}{c}6.26 \mathrm{E}- \\
03\end{array}$ & $\begin{array}{c}1.44 \mathrm{E}- \\
01\end{array}$ & $\begin{array}{c}6.93 \mathrm{E}- \\
01\end{array}$ & $\begin{array}{c}4.32 \mathrm{E}- \\
01\end{array}$ & 2 & PSAT1,PHGDH \\
\hline
\end{tabular}


medRxiv preprint doi: https://doi.org/10.1101/2020.12.21.20248688; this version posted December 24, 2020. The copyright holder for this preprint (which was not certified by peer review) is the author/funder, who has granted medRxiv a license to display the preprint in perpetuity. All rights reserved. No reuse allowed without permission.

\begin{tabular}{|c|c|c|c|c|c|c|c|}
\hline P00008 & $\begin{array}{c}\text { Axon guidance } \\
\text { mediated by } \\
\text { Slit/Robo }\end{array}$ & $\begin{array}{c}1.03 \mathrm{E}- \\
02\end{array}$ & $\begin{array}{c}1.78 \mathrm{E}- \\
01\end{array}$ & $\begin{array}{c}8.57 \mathrm{E}- \\
01\end{array}$ & $\begin{array}{c}7.11 \mathrm{E}- \\
01\end{array}$ & 3 & $\begin{array}{c}\text { SRGAP1,RHOC,R } \\
\text { AC2 }\end{array}$ \\
\hline P04378 & $\begin{array}{l}\text { Beta2 } \\
\text { adrenergic } \\
\text { receptor } \\
\text { signaling } \\
\text { pathway }\end{array}$ & $\begin{array}{l}2.41 \mathrm{E}- \\
02\end{array}$ & $\begin{array}{c}2.96 \mathrm{E}- \\
01\end{array}$ & $\begin{array}{c}1.00 \mathrm{E}+ \\
00\end{array}$ & $\begin{array}{c}1.00 \mathrm{E}+0 \\
0\end{array}$ & 4 & $\begin{array}{c}\text { RYR3,VAMP3,A } \\
\text { DRB2,VAMP8 }\end{array}$ \\
\hline P00034 & $\begin{array}{l}\text { Integrin } \\
\text { signalling } \\
\text { pathway }\end{array}$ & $\begin{array}{l}2.86 \mathrm{E}- \\
02\end{array}$ & $\begin{array}{c}2.96 \mathrm{E}- \\
01\end{array}$ & $\begin{array}{c}1.00 \mathrm{E}+ \\
00\end{array}$ & $\begin{array}{c}1.00 \mathrm{E}+0 \\
0\end{array}$ & 9 & $\begin{array}{c}\text { COL20A1,RHOC, } \\
\text { CSK,ITGAX,ITG } \\
\text { B1,ITGB2,ITGB4, } \\
\text { ITGB5,RAC2 }\end{array}$ \\
\hline P00037 & $\begin{array}{l}\text { Ionotropic } \\
\text { glutamate } \\
\text { receptor } \\
\text { pathway }\end{array}$ & $\begin{array}{c}3.00 \mathrm{E}- \\
02\end{array}$ & $\begin{array}{c}2.96 \mathrm{E}- \\
01\end{array}$ & $\begin{array}{c}1.00 \mathrm{E}+ \\
00\end{array}$ & $\begin{array}{c}1.00 \mathrm{E}+0 \\
0\end{array}$ & 4 & $\begin{array}{l}\text { VAMP3,SLC1A3, } \\
\text { VAMP8,GRIN2C }\end{array}$ \\
\hline
\end{tabular}

Pathway Ontology

\begin{tabular}{|c|c|c|c|c|c|c|c|}
\hline PW:0000286 & $\begin{array}{l}\text { integrin } \\
\text { signaling }\end{array}$ & $\begin{array}{c}4.41 \mathrm{E}- \\
05\end{array}$ & $\begin{array}{c}2.87 \mathrm{E}- \\
03\end{array}$ & $\begin{array}{c}1.37 \mathrm{E}- \\
02\end{array}$ & $\begin{array}{c}2.87 \mathrm{E}- \\
03\end{array}$ & 7 & $\begin{array}{l}\text { APBB1IP,PARVG } \\
\text {,ITGAX,ITGB1,IT } \\
\text { GB2,ITGB4,ITGB } \\
5\end{array}$ \\
\hline PW:0000484 & $\begin{array}{c}\text { altered } \\
\text { lipoprotein } \\
\text { metabolic }\end{array}$ & $\begin{array}{c}5.45 \mathrm{E}- \\
04\end{array}$ & $\begin{array}{l}1.77 \mathrm{E}- \\
02\end{array}$ & $\begin{array}{c}8.43 \mathrm{E}- \\
02\end{array}$ & $\begin{array}{l}3.54 \mathrm{E}- \\
02\end{array}$ & 3 & $\begin{array}{c}\mathrm{APOC} 1, \mathrm{APOC} 2, \mathrm{~A} \\
\text { POE }\end{array}$ \\
\hline PW:0000464 & $\begin{array}{l}\text { leukotriene } \\
\text { metabolic }\end{array}$ & $\begin{array}{c}3.82 \mathrm{E}- \\
03\end{array}$ & $\begin{array}{c}6.46 \mathrm{E}- \\
02\end{array}$ & $\begin{array}{c}3.07 \mathrm{E}- \\
01\end{array}$ & $\begin{array}{c}2.48 \mathrm{E}- \\
01\end{array}$ & 2 & $\begin{array}{c}\text { ALOX5,ALOX5A } \\
\text { P }\end{array}$ \\
\hline PW:0000482 & $\begin{array}{l}\text { lipoprotein } \\
\text { metabolic }\end{array}$ & $\begin{array}{l}3.97 \mathrm{E}- \\
03\end{array}$ & $\begin{array}{c}6.46 \mathrm{E}- \\
02\end{array}$ & $\begin{array}{c}3.07 \mathrm{E}- \\
01\end{array}$ & $\begin{array}{l}2.58 \mathrm{E}- \\
01\end{array}$ & 3 & $\begin{array}{c}\text { APOC } 1, \mathrm{APOC} 2, \mathrm{~A} \\
\text { POE }\end{array}$ \\
\hline PW:0000462 & $\begin{array}{l}\text { lipoxygenase } \\
\text { mediated of } \\
\text { arachidonic acid } \\
\text { metabolism }\end{array}$ & $\begin{array}{c}1.66 \mathrm{E}- \\
02\end{array}$ & $\begin{array}{c}1.43 \mathrm{E}- \\
01\end{array}$ & $\begin{array}{c}6.81 \mathrm{E}- \\
01\end{array}$ & $\begin{array}{c}1.00 \mathrm{E}+0 \\
0\end{array}$ & 2 & $\begin{array}{c}\text { ALOX5,ALOX5A } \\
\text { P }\end{array}$ \\
\hline PW:0000228 & $\begin{array}{c}\text { G protein } \\
\text { signaling via } \\
\text { Galphai family }\end{array}$ & $\begin{array}{l}2.10 \mathrm{E}- \\
02\end{array}$ & $\begin{array}{c}1.43 \mathrm{E}- \\
01\end{array}$ & $\begin{array}{c}6.81 \mathrm{E}- \\
01\end{array}$ & $\begin{array}{c}1.00 \mathrm{E}+0 \\
0\end{array}$ & 2 & ADRB2,LPAR1 \\
\hline PW:0000498 & $\begin{array}{c}\text { reverse } \\
\text { cholesterol }\end{array}$ & $2.59 \mathrm{E}-$ & $1.43 \mathrm{E}-$ & $6.81 \mathrm{E}-$ & $1.00 \mathrm{E}+0$ & 2 & ABCA1,PLTP \\
\hline
\end{tabular}


$\begin{array}{lllll}\text { transport } & 02 & 01 & 01 & 0\end{array}$

\begin{tabular}{|c|c|c|c|c|c|c|c|}
\hline PW:0000234 & $\begin{array}{l}\text { innate immune } \\
\text { response }\end{array}$ & $\begin{array}{c}3.11 \mathrm{E}- \\
02\end{array}$ & $\begin{array}{c}1.50 \mathrm{E}- \\
01\end{array}$ & $\begin{array}{c}7.14 \mathrm{E}- \\
01\end{array}$ & $\begin{array}{c}1.00 \mathrm{E}+0 \\
0\end{array}$ & 2 & CD14,TLR5 \\
\hline PW:0000233 & $\begin{array}{l}\text { tumor necrosis } \\
\text { factor mediated } \\
\text { signaling }\end{array}$ & $\begin{array}{c}3.67 \mathrm{E}- \\
02\end{array}$ & $\begin{array}{l}1.50 \mathrm{E}- \\
01\end{array}$ & $\begin{array}{c}7.14 \mathrm{E}- \\
01\end{array}$ & $\begin{array}{c}1.00 \mathrm{E}+0 \\
0\end{array}$ & 2 & $\begin{array}{c}\text { ADAM17,TNFRS } \\
\text { F1A }\end{array}$ \\
\hline PW:0000043 & $\begin{array}{l}\text { pyruvate } \\
\text { metabolic }\end{array}$ & $\begin{array}{c}4.09 \mathrm{E}- \\
02\end{array}$ & $\begin{array}{c}1.50 \mathrm{E}- \\
01\end{array}$ & $\begin{array}{c}7.14 \mathrm{E}- \\
01\end{array}$ & $\begin{array}{c}1.00 \mathrm{E}+0 \\
0\end{array}$ & 3 & $\mathrm{ACACB}, \mathrm{PDK} 4, \mathrm{PC}$ \\
\hline
\end{tabular}

\section{SMPDB}

\begin{tabular}{|c|c|c|c|c|c|c|c|}
\hline SMP00313 & Lactic Acidemia & $\begin{array}{l}2.64 \mathrm{E}- \\
02\end{array}$ & $\begin{array}{l}4.84 \mathrm{E}- \\
01\end{array}$ & $\begin{array}{c}1.00 \mathrm{E}+ \\
00\end{array}$ & $\begin{array}{c}1.00 \mathrm{E}+0 \\
0\end{array}$ & 1 & $\mathrm{PC}$ \\
\hline SMP00319 & $\begin{array}{c}\text { Lysosomal Acid } \\
\text { Lipase } \\
\text { Deficiency } \\
\text { (Wolman } \\
\text { Disease) }\end{array}$ & $\begin{array}{l}2.64 \mathrm{E}- \\
02\end{array}$ & $\begin{array}{c}4.84 \mathrm{E}- \\
01\end{array}$ & $\begin{array}{c}1.00 \mathrm{E}+ \\
00\end{array}$ & $\begin{array}{c}1.00 \mathrm{E}+0 \\
0\end{array}$ & 1 & LIPA \\
\hline SMP00360 & $\begin{array}{c}\text { Hyperprolinemi } \\
\text { a Type II }\end{array}$ & $\begin{array}{c}2.64 \mathrm{E}- \\
02\end{array}$ & $\begin{array}{c}4.84 \mathrm{E}- \\
01\end{array}$ & $\begin{array}{c}1.00 \mathrm{E}+ \\
00\end{array}$ & $\begin{array}{c}1.00 \mathrm{E}+0 \\
0\end{array}$ & 1 & ALDH4A1 \\
\hline SMP00208 & $\begin{array}{c}\text { Prolinemia Type } \\
\text { II }\end{array}$ & $\begin{array}{c}2.64 \mathrm{E}- \\
02\end{array}$ & $\begin{array}{c}4.84 \mathrm{E}- \\
01\end{array}$ & $\begin{array}{c}1.00 \mathrm{E}+ \\
00\end{array}$ & $\begin{array}{c}1.00 \mathrm{E}+0 \\
0\end{array}$ & 1 & ALDH4A1 \\
\hline SMP00202 & $\begin{array}{c}\text { MNGIE } \\
\text { (Mitochondrial } \\
\text { Neurogastrointe } \\
\text { stinal } \\
\text { Encephalopathy) }\end{array}$ & $\begin{array}{l}2.64 \mathrm{E}- \\
02\end{array}$ & $\begin{array}{c}4.84 \mathrm{E}- \\
01\end{array}$ & $\begin{array}{c}1.00 \mathrm{E}+ \\
00\end{array}$ & $\begin{array}{c}1.00 \mathrm{E}+0 \\
0\end{array}$ & 1 & TYMP \\
\hline SMP00350 & $\begin{array}{c}\text { Pyruvate } \\
\text { Carboxylase } \\
\text { Deficiency }\end{array}$ & $\begin{array}{l}2.64 \mathrm{E}- \\
02\end{array}$ & $\begin{array}{c}4.84 \mathrm{E}- \\
01\end{array}$ & $\begin{array}{c}1.00 \mathrm{E}+ \\
00\end{array}$ & $\begin{array}{c}1.00 \mathrm{E}+0 \\
0\end{array}$ & 1 & $\mathrm{PC}$ \\
\hline
\end{tabular}

Table 4 The enriched GO terms of the up regulated differentially expressed genes

\begin{tabular}{ccccccccc} 
GO ID & $\begin{array}{c}\text { CATEGO } \\
\text { RY }\end{array}$ & $\begin{array}{c}\text { GO } \\
\text { Name }\end{array}$ & $\begin{array}{c}\text { P } \\
\text { Value }\end{array}$ & $\begin{array}{c}\text { FDR } \\
\text { B\&H }\end{array}$ & $\begin{array}{c}\text { FDR } \\
\text { B\&Y }\end{array}$ & $\begin{array}{c}\text { Bonferr } \\
\text { oni }\end{array}$ & $\begin{array}{c}\text { Gene } \\
\text { Cou } \\
\text { nt }\end{array}$ & Gene \\
\hline GO:0007 & BP & & & & & & & \\
\hline & & chemical & $8.37 \mathrm{E}-$ & $1.17 \mathrm{E}-$ & $1.04 \mathrm{E}-$ & $3.51 \mathrm{E}-$ & 69 & RTN3,PFN2,CHR \\
& & & & & & & M1,ADCYAP1,SL
\end{tabular}


medRxiv preprint doi: https://doi.org/10.1101/2020.12.21.20248688; this version posted December 24, 2020. The copyright holder for this preprint (which was not certified by peer review) is the author/funder, who has granted medRxiv a license to display the preprint in perpetuity. All rights reserved. No reuse allowed without permission.

268

GO:0031

175 transmiss

ion
24

23

24

ion

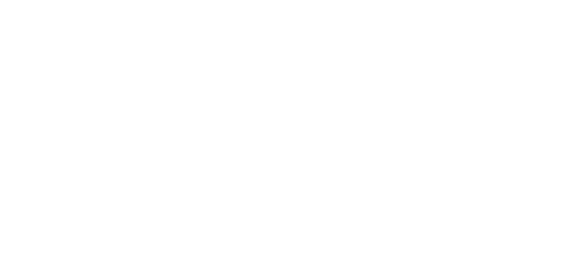

$\begin{array}{ccccc}\text { neuron } & 3.51 \mathrm{E}- & 2.94 \mathrm{E}- & 2.62 \mathrm{E}- & 1.47 \mathrm{E}- \\ \text { projectio } & 23 & 20 & 19 & 19 \\ \mathrm{n} & & & & \\ \text { develop } & & & & \\ \text { ment } & & & & \end{array}$

\section{C12A5,CACNG3, NRXN1,LRFN5,S CN1B,SCN2B,FGF 12,SYT13,VGF,A MPH,ICA1,ADGR L1,GABBR2,PNK \\ D,YWHAH,SLC32 A1,NPTN,SLC8A2 ,PPP3CB,NAPB,P RKCE,PRKCG,SN AP25,SNCB,SNCA ,CADPS2,ATP2A2, ATP2B2,GABRA1, GABRB3,GABRD, GABRG2,GAD1,G AD2,CLSTN3,CPL X2,CPLX1,LRRT M1,SST,LIN7B,ST X1A,STXBP1,PTP RN2,SV2B,GLRB, GLS,VAMP2,SYN 1,SYN2,SYP,SYT1 ,TAC1,DNM1,NPY ,CACNB2,NPTX1, NRGN,KALRN,C AMK2B,CCKBR,R IT2,EGR1,SYNGR 1,PAK1,CDK5}

PCP4,MAP1B,MA
P2,MAP4,BCL11A
,NREP,CHN1,ADC
Y1,SLITRK1,ADC
YAP1,SLC12A5,S
LIT2,TUBB3,NRX
N1,LRFN5,UCHL1
,HPRT1,SCN1B,N
GEF,OLFM1,FLR
T3,SLC9A6,PACSI
N1,ATCAY,CNTN
AP1,ENC1,GAS7,
XK,APP,YWHAH,
NPTN,PPP3CB,CA
PRIN2,CNTNAP2,
75

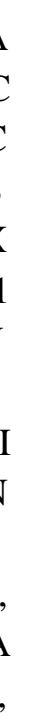


medRxiv preprint doi: https://doi.org/10.1101/2020.12.21.20248688; this version posted December 24, 2020. The copyright holder for this preprint (which was not certified by peer review) is the author/funder, who has granted medRxiv a license to display the preprint in perpetuity. All rights reserved. No reuse allowed without permission.

GO:0007

267

\begin{abstract}
BP
\end{abstract}

\begin{abstract}
cell-cell
\end{abstract}

(1)

$1.84 \mathrm{E}-\quad 1.28 \mathrm{E}-\quad 1.14 \mathrm{E}-\quad 7.70 \mathrm{E}-$

22

19
SNAP25,TBR1,M

APK9,MAP2K1,R

AP1GAP2,EPB41L

3,NEFM,NEFH,NE

FL,PRDM8,STXB

P1,PLPPR4,PTPR

M,BHLHE22,SYT

1,KIF3C,GNAO1, HERC1,NME1,NP Y,GPM6A,NPTX1, CAMK1D,B4GAT

1,KALRN,FZD3,C

AMK2B,RTN4,SLI

TRK4,STMN2,ST

MN1,OPA1,CCK,L

INGO1,NPTXR,PA

K1,ELAVL4,DCL

K1,RBFOX2,EPH A4,CDK5

85

PCSK1,RTN3,PFN

2,CHRM1,ADCYA P1,SLC12A5,CAC NG3,NRXN1,LRF N5,SCN1B,SCN2B ,SCN4B,FGF9,FGF 12,SYT13,VGF,A MPH,VSNL1,SCG 5,ICA1,MYRIP,AD GRL1,SYBU,GAB BR2,PNKD,YWH AH,YWHAZ,SLC3 2A1,NPTN,SLC8A 2,PPP3CB,NAPB, VPS35,PRKCE,PR KCG,SNAP25,SN CB,SNCA,CADPS 2,ATP2A2,ATP2B 2,SCN3B,GABRA 1,GABRB3,GABR D,GABRG2,GAD1 ,GAD2,CLSTN3,C PLX2,CPLX1,LRR TM1,SST,ITPR1,LI 
medRxiv preprint doi: https://doi.org/10.1101/2020.12.21.20248688; this version posted December 24, 2020. The copyright holder for this preprint (which was not certified by peer review) is the author/funder, who has granted medRxiv a license to display the preprint in perpetuity. All rights reserved. No reuse allowed without permission.

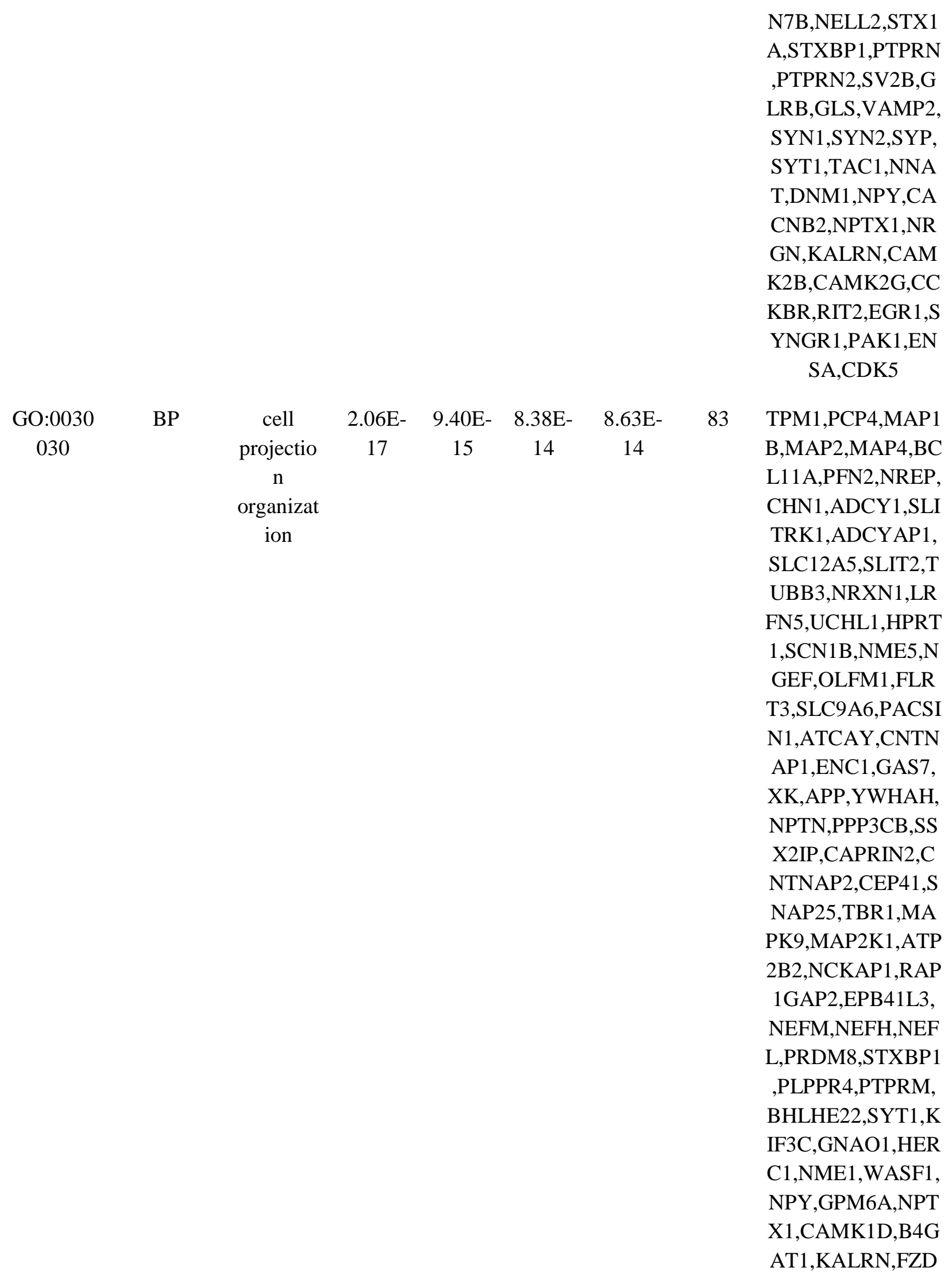


medRxiv preprint doi: https://doi.org/10.1101/2020.12.21.20248688; this version posted December 24, 2020. The copyright holder for this preprint (which was not certified by peer review) is the author/funder, who has granted medRxiv a license to display the preprint in perpetuity. All rights reserved. No reuse allowed without permission.

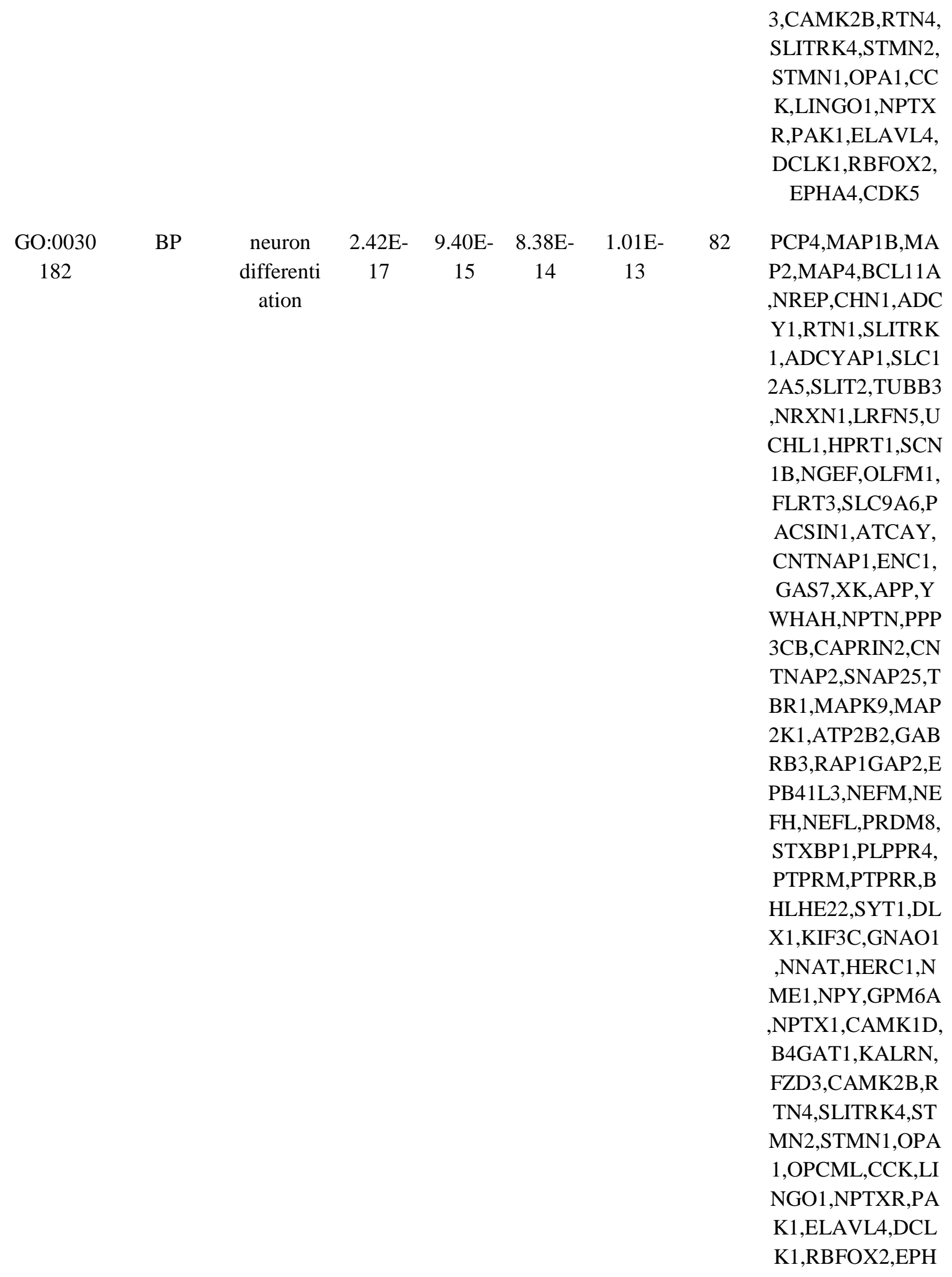


medRxiv preprint doi: https://doi.org/10.1101/2020.12.21.20248688; this version posted December 24, 2020. The copyright holder for this preprint (which was not certified by peer review) is the author/funder, who has granted medRxiv a license to display the preprint in perpetuity. All rights reserved. No reuse allowed without permission.

\begin{tabular}{|c|c|c|c|c|c|c|c|c|}
\hline & & & & & & & & $\mathrm{A} 4, \mathrm{CDK} 5$ \\
\hline $\begin{array}{c}\text { GO:0006 } \\
811\end{array}$ & BP & $\begin{array}{c}\text { ion } \\
\text { transport }\end{array}$ & $\begin{array}{c}1.02 \mathrm{E}- \\
12\end{array}$ & $\begin{array}{c}1.34 \mathrm{E}- \\
10\end{array}$ & $\begin{array}{c}1.20 \mathrm{E}- \\
09\end{array}$ & $\begin{array}{c}4.29 \mathrm{E}- \\
09\end{array}$ & 80 & $\begin{array}{c}\text { PCP4,NCALD,CH } \\
\text { RM1,SLC22A17,A } \\
\text { DCYAP1,SLC12A } \\
\text { 5,CACNG3,NRXN } \\
\text { 1,SCN1B,SCN2B,S } \\
\text { CN4B,FGF12,FKB } \\
\text { P1A,SLC9A6,AKA } \\
\text { P6,DPP10,XK,YW } \\
\text { HAH,YWHAZ,SL } \\
\text { C32A1,SLC8A2,A } \\
\text { TP6V1H,SLC25A1 } \\
\text { 2,GNB5,PRKCB,K } \\
\text { CNIP4,PRKCE,SN } \\
\text { AP25,ATP1A1,SN } \\
\text { CA,ATP1B1,MAP } \\
\text { K9,ATP2A2,ATP2 } \\
\text { B1,ATP2B2,ATP2 } \\
\text { B3,PRNP,SCN3B, } \\
\text { GABRA1,GABRB } \\
\text { 3,GABRD,GABRG } \\
\text { 2,ATP6V1A,ATP6 } \\
\text { V1B2,ATP6V0C,A } \\
\text { TP6V1E1,ATP6V1 } \\
\text { G2,GLS2,SLC6A1 } \\
\text { 7,CPLX1,STEAP2, } \\
\text { SLC30A3,ITPR1,S } \\
\text { LC38A1,STX1A,S } \\
\text { TXBP1,KCNJ4,GL } \\
\text { RB,KCNJ12,GLS, } \\
\text { VAMP2,SYT1,GN } \\
\text { AO1,NPY,SLC17A } \\
\text { 6,DENND5B,GPM } \\
\text { 6A,DPP6,CACNB2 } \\
\text {,CALM3,NSF,CA } \\
\text { MK2B,CAMK2G, } \\
\text { RTN4,TUSC3,SLC } \\
\text { 6A15,CCK,CCKB } \\
\text { RAKDK5 }\end{array}$ \\
\hline $\begin{array}{c}\text { GO:0051 } \\
049\end{array}$ & BP & $\begin{array}{l}\text { regulatio } \\
\text { n of } \\
\text { transport }\end{array}$ & $\begin{array}{c}7.88 \mathrm{E}- \\
11\end{array}$ & $\begin{array}{c}8.46 \mathrm{E}- \\
09\end{array}$ & $\begin{array}{c}7.55 \mathrm{E}- \\
08\end{array}$ & $\begin{array}{c}3.30 \mathrm{E}- \\
07\end{array}$ & 86 & $\begin{array}{l}\text { PCP4,MAP1B,CH } \\
\text { GA,PFN2,CHRM1, } \\
\text { ADCYAP1,CACN } \\
\text { G3,HPCA,NRXN1, }\end{array}$ \\
\hline
\end{tabular}


medRxiv preprint doi: https://doi.org/10.1101/2020.12.21.20248688; this version posted December 24, 2020. The copyright holder for this preprint (which was not certified by peer review) is the author/funder, who has granted medRxiv a license to display the preprint in perpetuity. All rights reserved. No reuse allowed without permission.

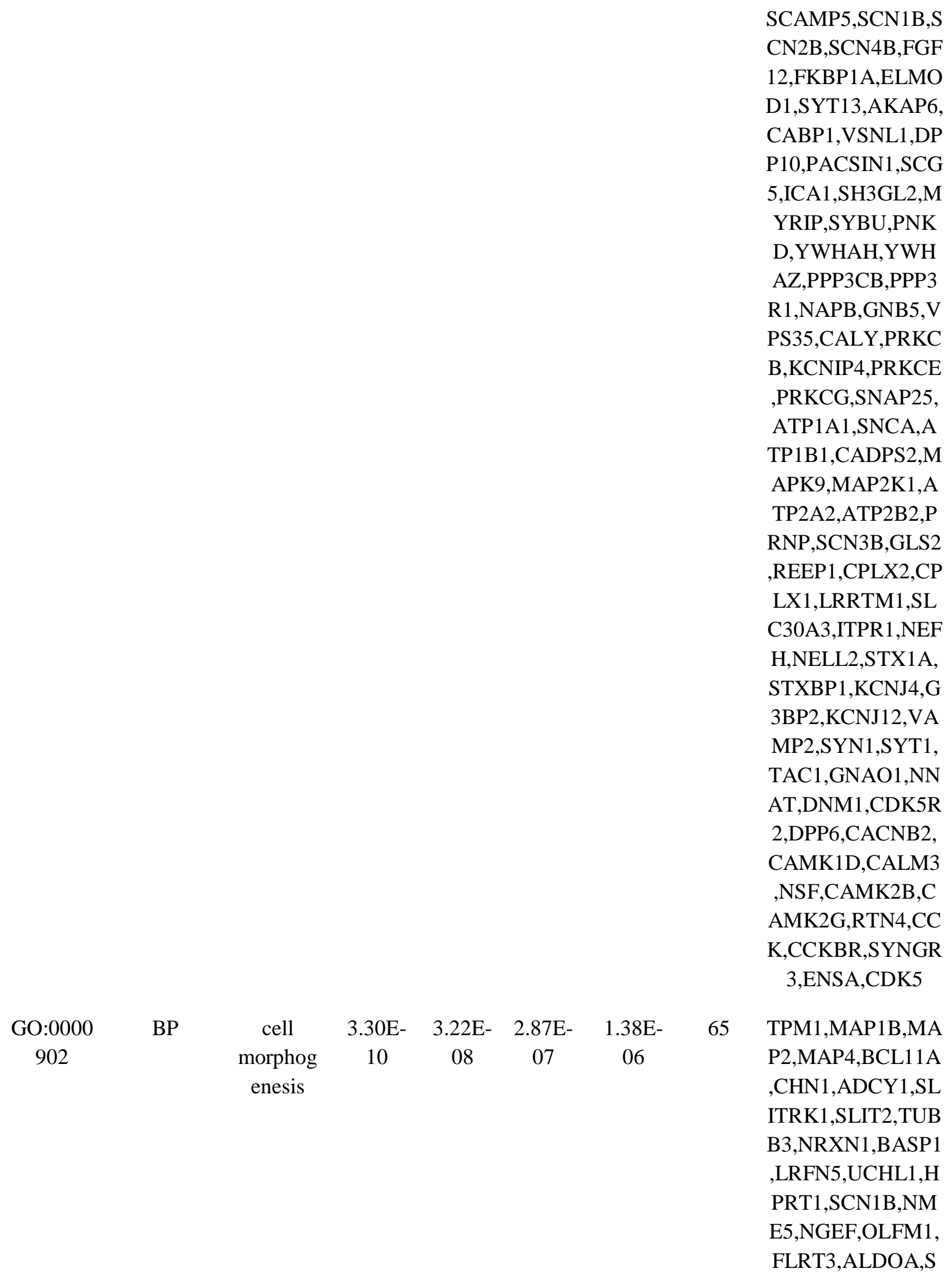


medRxiv preprint doi: https://doi.org/10.1101/2020.12.21.20248688; this version posted December 24, 2020. The copyright holder for this preprint (which was not certified by peer review) is the author/funder, who has granted medRxiv a license to display the preprint in perpetuity. All rights reserved. No reuse allowed without permission.

\begin{tabular}{|c|c|c|c|c|c|c|c|c|}
\hline $\begin{array}{c}\text { GO:0016 } \\
192\end{array}$ & BP & $\begin{array}{l}\text { vesicle- } \\
\text { mediated } \\
\text { transport }\end{array}$ & $\begin{array}{c}1.01 \mathrm{E}- \\
07\end{array}$ & $\begin{array}{c}5.00 \mathrm{E}- \\
06\end{array}$ & $\begin{array}{c}4.45 \mathrm{E}- \\
05\end{array}$ & $\begin{array}{c}4.25 \mathrm{E}- \\
04\end{array}$ & 64 & $\begin{array}{c}\text { NCALD,RTN3,CH } \\
\text { GA,PFN2,AP1S1,N } \\
\text { RXN1,SCAMP5,F } \\
\text { AM3C,ALDOA,SY } \\
\text { T13,AMPH,VSNL } \\
\text { 1,PACSIN1,SH3G } \\
\text { L2,ADGRL1,APP, } \\
\text { RAB6B,YWHAZ, } \\
\text { CYFIP2,ARF3,NE } \\
\text { CAP1,ATP6V1H,P } \\
\text { PP3CB,NSG2,NAP } \\
\text { B,NSG1,VPS35,C } \\
\text { ALY,PRKCE,PRK } \\
\text { CG,SNAP25,SNC } \\
\text { A,CADPS2,MAP2 } \\
\text { K1,ATP2A2,NCK } \\
\text { AP1,CPLX2,CPLX } \\
\text { 1,LRRTM1,STEAP } \\
\text { 2,ELMO1,LIN7B,S } \\
\text { TX1A,STXBP1,AR } \\
\text { HGAP44,VAMP1, } \\
\text { VAMP2,SYN1,SY } \\
\text { P,SYT1,KIF3C,NM }\end{array}$ \\
\hline
\end{tabular}


medRxiv preprint doi: https://doi.org/10.1101/2020.12.21.20248688; this version posted December 24, 2020. The copyright holder for this preprint (which was not certified by peer review) is the author/funder, who has granted medRxiv a license to display the preprint in perpetuity. All rights reserved. No reuse allowed without permission.

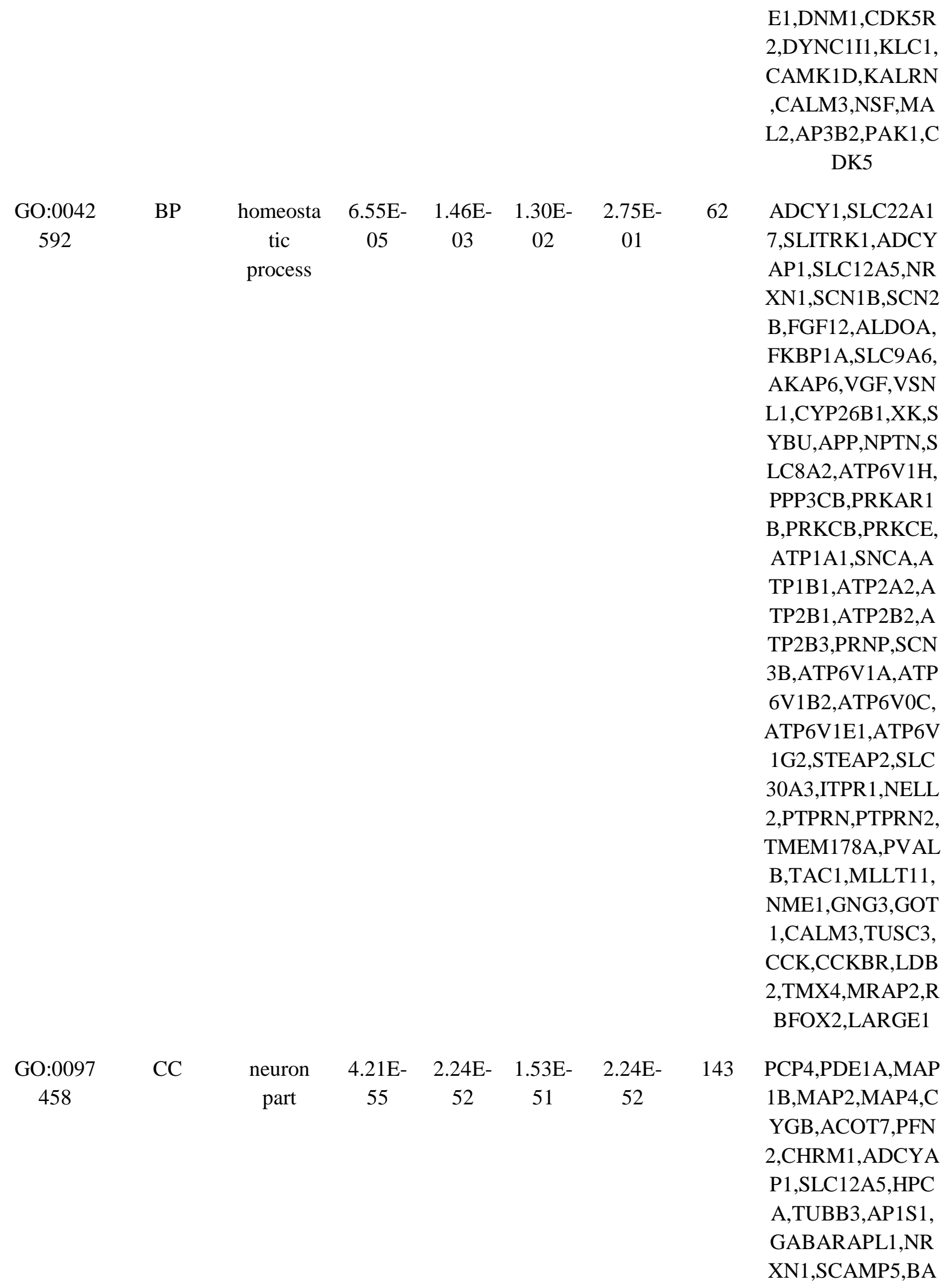


medRxiv preprint doi: https://doi.org/10.1101/2020.12.21.20248688; this version posted December 24, 2020. The copyright holder for this preprint (which was not certified by peer review) is the author/funder, who has granted medRxiv a license to display the preprint in perpetuity. All rights reserved. No reuse allowed without permission.

SP1,UCHL1,SCN1
B,NGEF,OLFM1,F
LRT3,FKBP1A,SV
OP,SLC9A6,SYT1
3,CAP2,VGF,MAP
2K4,AMPH,PACSI
N1,ATCAY,ICA1,
CNTNAP1,ENC1,
MYRIP,ADGRL1,
MAGEE1,APP,GA
BBR2,CYFIP2,PPP
1R2,SLC32A1,NP
TN,SLC8A2,GNB5
,VPS35,CNTNAP2,
ERC2,KCNIP4,PR
KCG,SNAP25,ATP
1A1,SNCB,TBR1,
NRSN1,SNCA,CA
DPS2,MAPK9,MA
P2K1,ATP2B1,AT
P2B2,GABRB3,G
ABRG2,GAD1,GA
D2,ARHGAP32,N
MNAT2,CLSTN3,
ATP6V1G2,FRMP
D4,SLC6A17,KCT
D8,CPLX2,CPLX1,
RAP1GAP2,LAMP
5,LRRTM1,SST,EP
B41L3,SLC30A3,I
TPR1,SLC38A1,LI
N7B,NEFM,NEFH,
NEFL,NELL2,STX
1A,STXBP1,PTPR
N,PTPRN2,SV2B,
KCNJ4,GLRB,KC
NJ12,PVALB,ARH
GAP44,VAMP1,V
AMP2,SYN1,SYN
2,SYP,SYT1,TAC1
,KIF3C,GNAO1,D
NM1,GNG3,NPY,
7A6,GPM6A,DPP6 
medRxiv preprint doi: https://doi.org/10.1101/2020.12.21.20248688; this version posted December 24, 2020. The copyright holder for this preprint (which was not certified by peer review) is the author/funder, who has granted medRxiv a license to display the preprint in perpetuity. All rights reserved. No reuse allowed without permission.

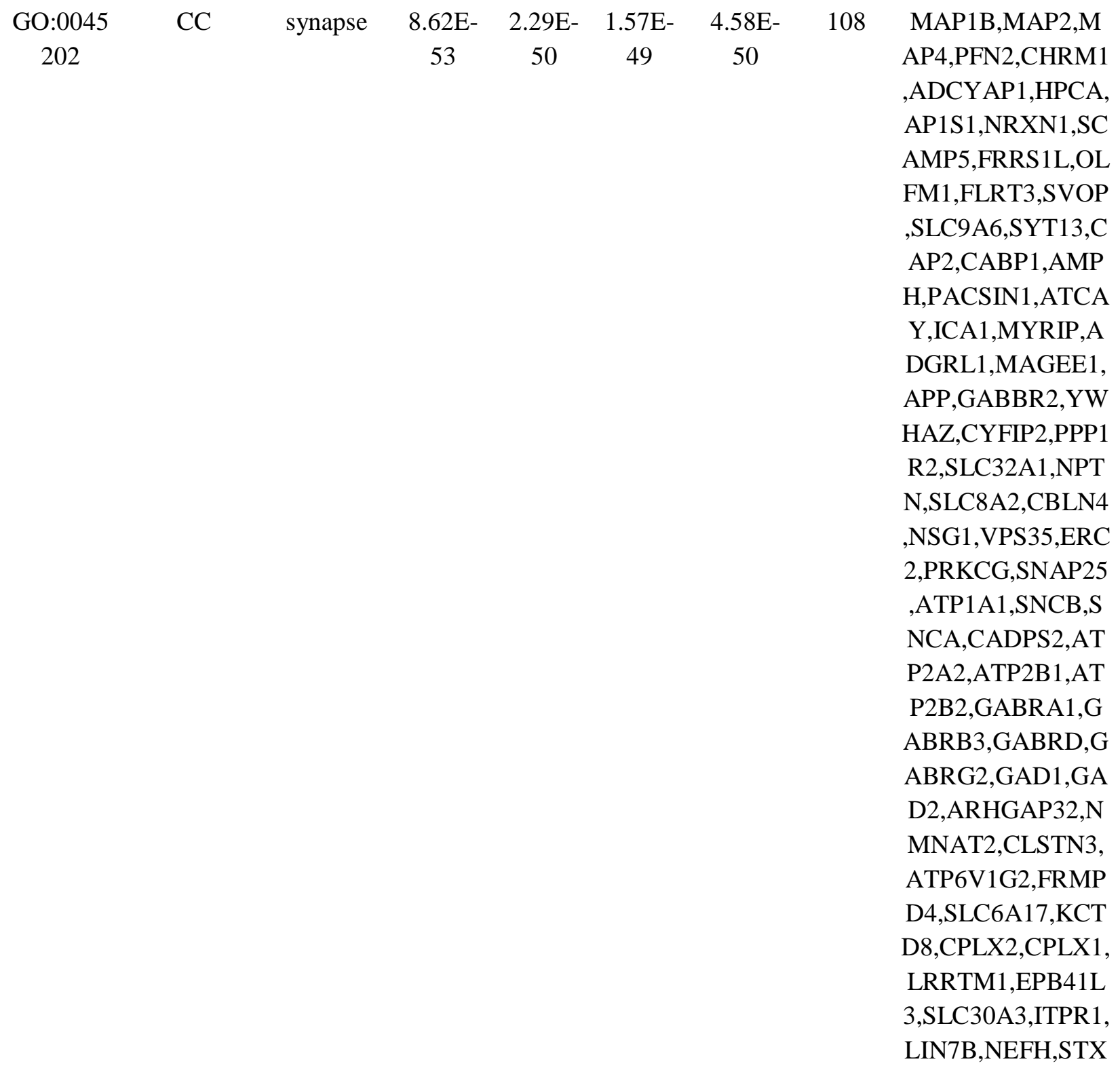


medRxiv preprint doi: https://doi.org/10.1101/2020.12.21.20248688; this version posted December 24, 2020. The copyright holder for this preprint (which was not certified by peer review) is the author/funder, who has granted medRxiv a license to display the preprint in perpetuity. All rights reserved. No reuse allowed without permission.

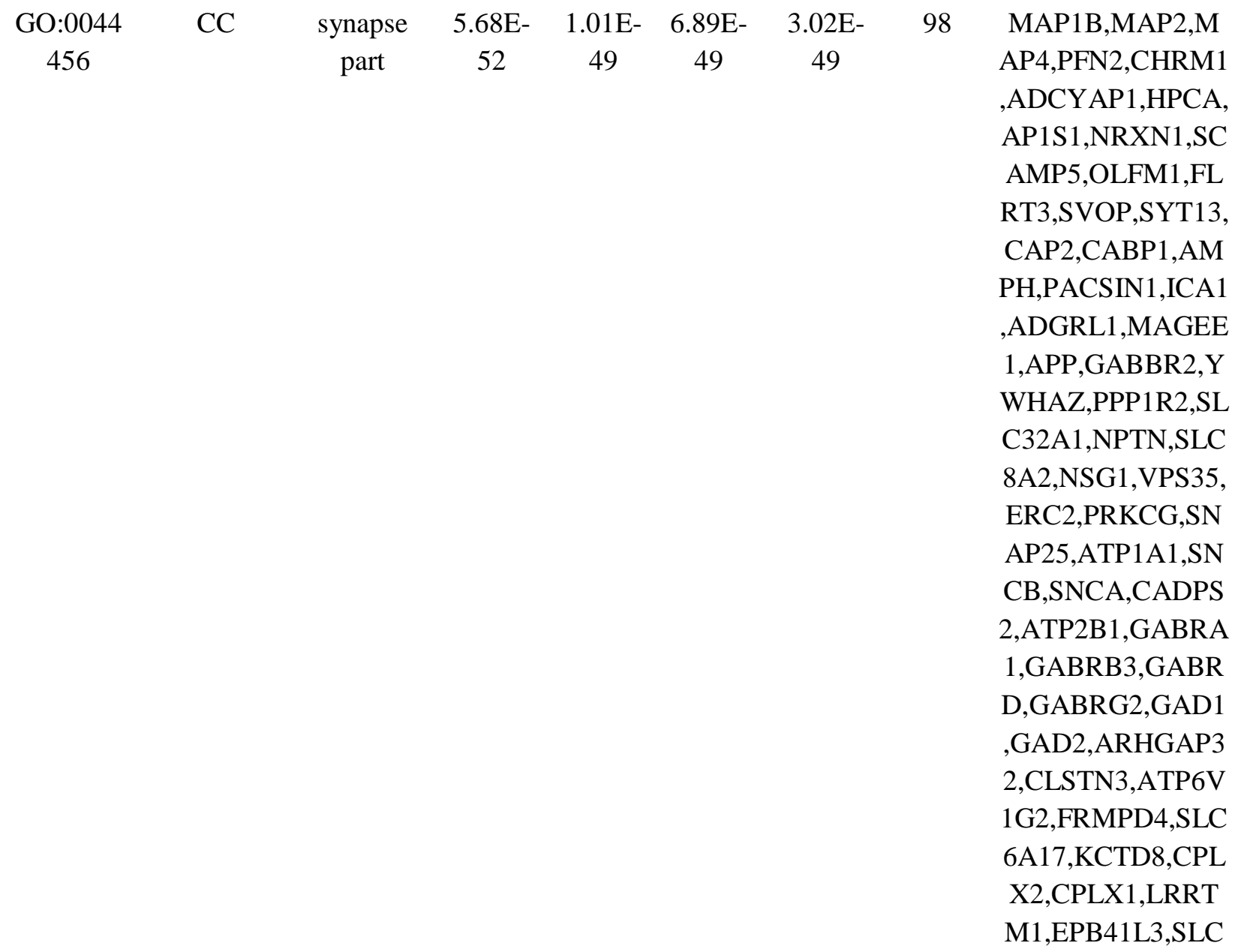


medRxiv preprint doi: https://doi.org/10.1101/2020.12.21.20248688; this version posted December 24, 2020. The copyright holder for this preprint (which was not certified by peer review) is the author/funder, who has granted medRxiv a license to display the preprint in perpetuity. All rights reserved. No reuse allowed without permission.

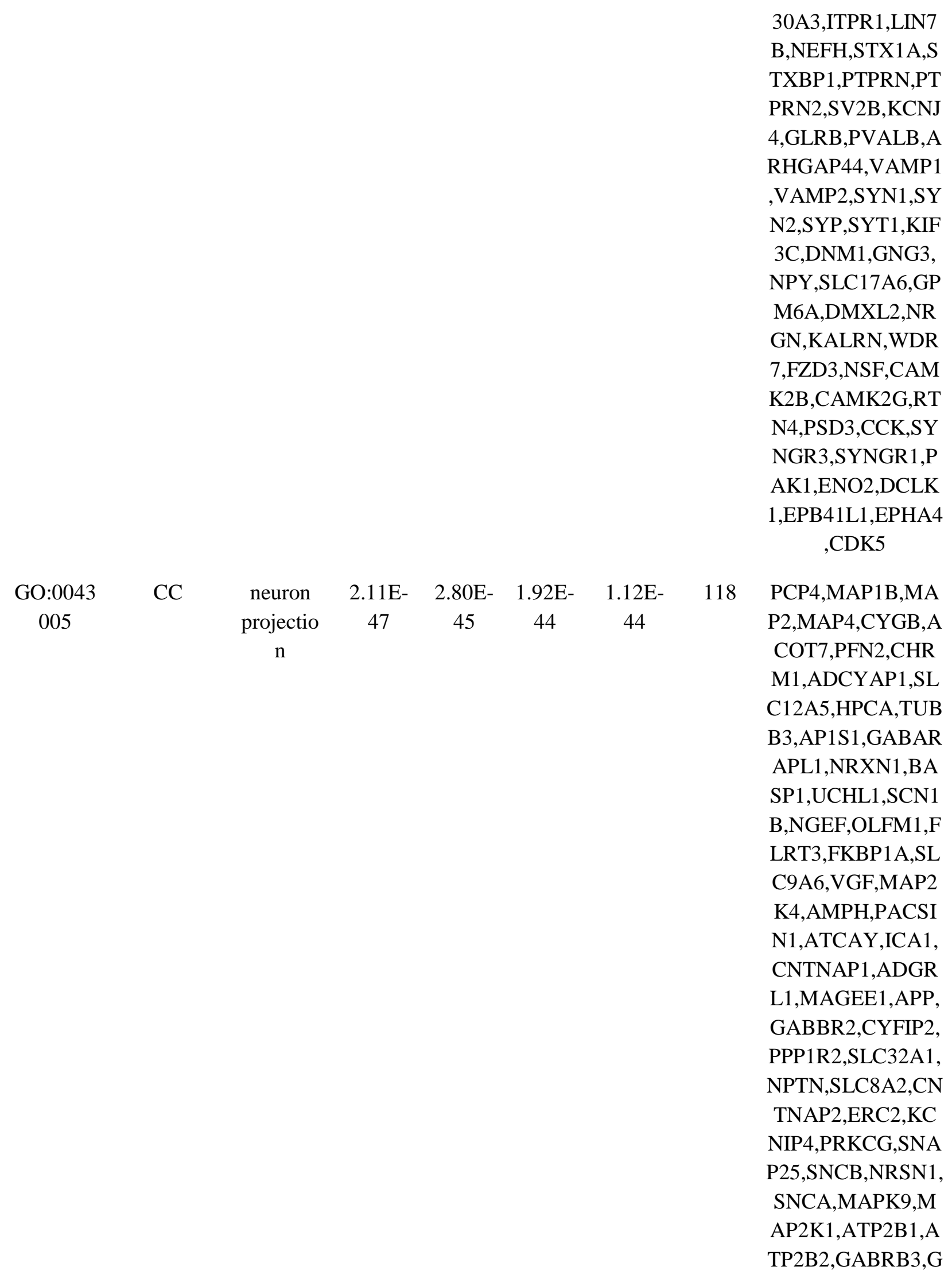


medRxiv preprint doi: https://doi.org/10.1101/2020.12.21.20248688; this version posted December 24, 2020. The copyright holder for this preprint (which was not certified by peer review) is the author/funder, who has granted medRxiv a license to display the preprint in perpetuity. All rights reserved. No reuse allowed without permission.

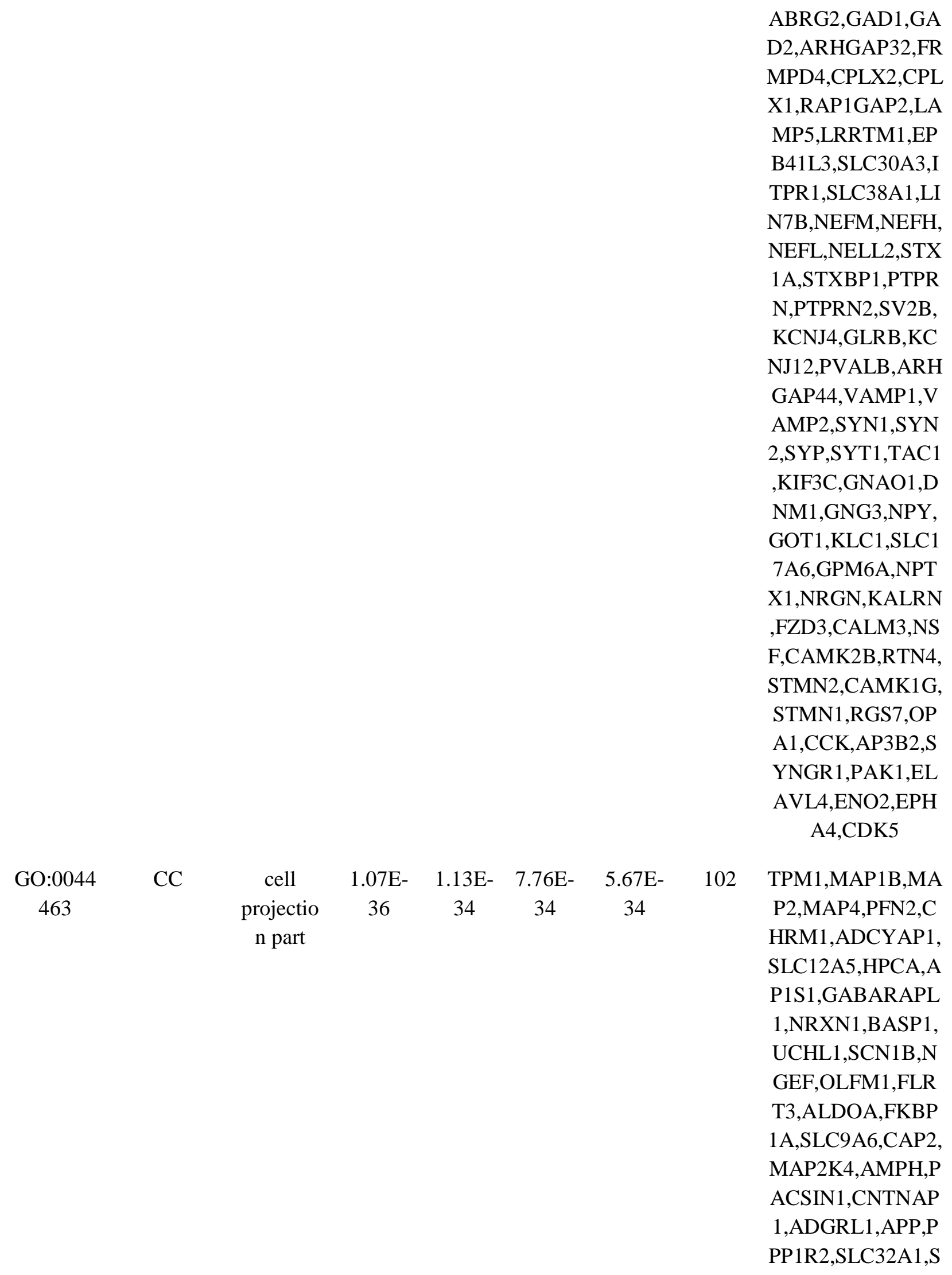


medRxiv preprint doi: https://doi.org/10.1101/2020.12.21.20248688; this version posted December 24, 2020. The copyright holder for this preprint (which was not certified by peer review) is the author/funder, who has granted medRxiv a license to display the preprint in perpetuity. All rights reserved. No reuse allowed without permission.

LC8A2,VPS35,SS

X2IP,CNTNAP2,C

EP41,PRKAR1B,P

RKCB,ERC2,PRK

CG,SNAP25,ATP1

A1,SNCB,NRSN1, SNCA,MAP2K1,A TP2B1,GABRB3,G ABRG2,GAD1,AR HGAP32,CLSTN3, FRMPD4,NCKAP1 ,CPLX2,CPLX1,L AMP5,LRRTM1,E PB41L3,NEFH,NE FL,STXBP1,PTPR N,PTPRN2,SV2B,P VALB,ARHGAP44 ,VAMP1,VAMP2,P DXP,SYN1,SYN2, SYP,SYT1,KIF3C, NME1,DNM1,GN G3,NPY,GOT1,KL C1,SLC17A6,GPM 6A,NRGN,KALRN ,FZD3,CALM3,NS F,CAMK2B,CAM K2G,RTN4,STMN 2,RGS7,OPA1,CC K,AP3B2,SYNGR1 ,PAK1,ELAVL4,D CLK1,EPB41L1,E PHA4,CDK5

GO:0030 CC 424 axon 1.84
33 $1.63 \mathrm{E}-1.12 \mathrm{E}-$ 31 30

PCP4,MAP1B,PFN 2,CHRM1,ADCYA P1,HPCA,TUBB3, AP1S1,NRXN1,UC HL1,SCN1B, OLF M1,FLRT3,FKBP1 A,SLC9A6,MAP2 K4,AMPH,PACSI N1,ATCAY,CNTN AP1,ADGRL1,APP ,SLC32A1,CNTNA 
medRxiv preprint doi: https://doi.org/10.1101/2020.12.21.20248688; this version posted December 24, 2020. The copyright holder for this preprint (which was not certified by peer review) is the author/funder, who has granted medRxiv a license to display the preprint in perpetuity. All rights reserved. No reuse allowed without permission.

\begin{tabular}{|c|c|c|c|c|c|c|c|c|}
\hline $\begin{array}{c}\text { GO:0036 } \\
477\end{array}$ & $\mathrm{CC}$ & $\begin{array}{l}\text { somatode } \\
\text { ndritic } \\
\text { compart } \\
\text { ment }\end{array}$ & $\begin{array}{c}5.47 \mathrm{E}- \\
25\end{array}$ & $\begin{array}{c}2.90 \mathrm{E}- \\
23\end{array}$ & $\begin{array}{c}1.99 \mathrm{E}- \\
22\end{array}$ & $\begin{array}{c}2.90 \mathrm{E}- \\
22\end{array}$ & 74 & $\begin{array}{c}\text { PDE1A,MAP1B,M } \\
\text { AP2,CYGB,CHRM } \\
\text { 1,SLC12A5,HPCA, } \\
\text { TUBB3,GABARA } \\
\text { PL1,NRXN1,UCH } \\
\text { L1,OLFM1,SLC9A } \\
\text { 6,VGF,MAP2K4,A } \\
\text { TCAY,ICA1,ENC1 } \\
\text {,MAGEE1,APP,PP } \\
\text { P1R2,SLC32A1,NP } \\
\text { TN,SLC8A2,CNT } \\
\text { NAP2,ERC2,KCNI } \\
\text { P4,PRKCG,SNAP2 } \\
\text { 5,SNCB,NRSN1,M } \\
\text { APK9,MAP2K1,A } \\
\text { TP2B1,ATP2B2,A } \\
\text { RHGAP32,FRMPD } \\
\text { 4,CPLX2,CPLX1,L } \\
\text { AMP5,SST,ITPR1, } \\
\text { NEFH,NELL2,PTP } \\
\text { RN,KCNJ4,GLRB, } \\
\text { KCNJ12,PVALB,A } \\
\text { RHGAP44,SYN1,T }\end{array}$ \\
\hline
\end{tabular}


medRxiv preprint doi: https://doi.org/10.1101/2020.12.21.20248688; this version posted December 24, 2020. The copyright holder for this preprint (which was not certified by peer review) is the author/funder, who has granted medRxiv a license to display the preprint in perpetuity. All rights reserved. No reuse allowed without permission.

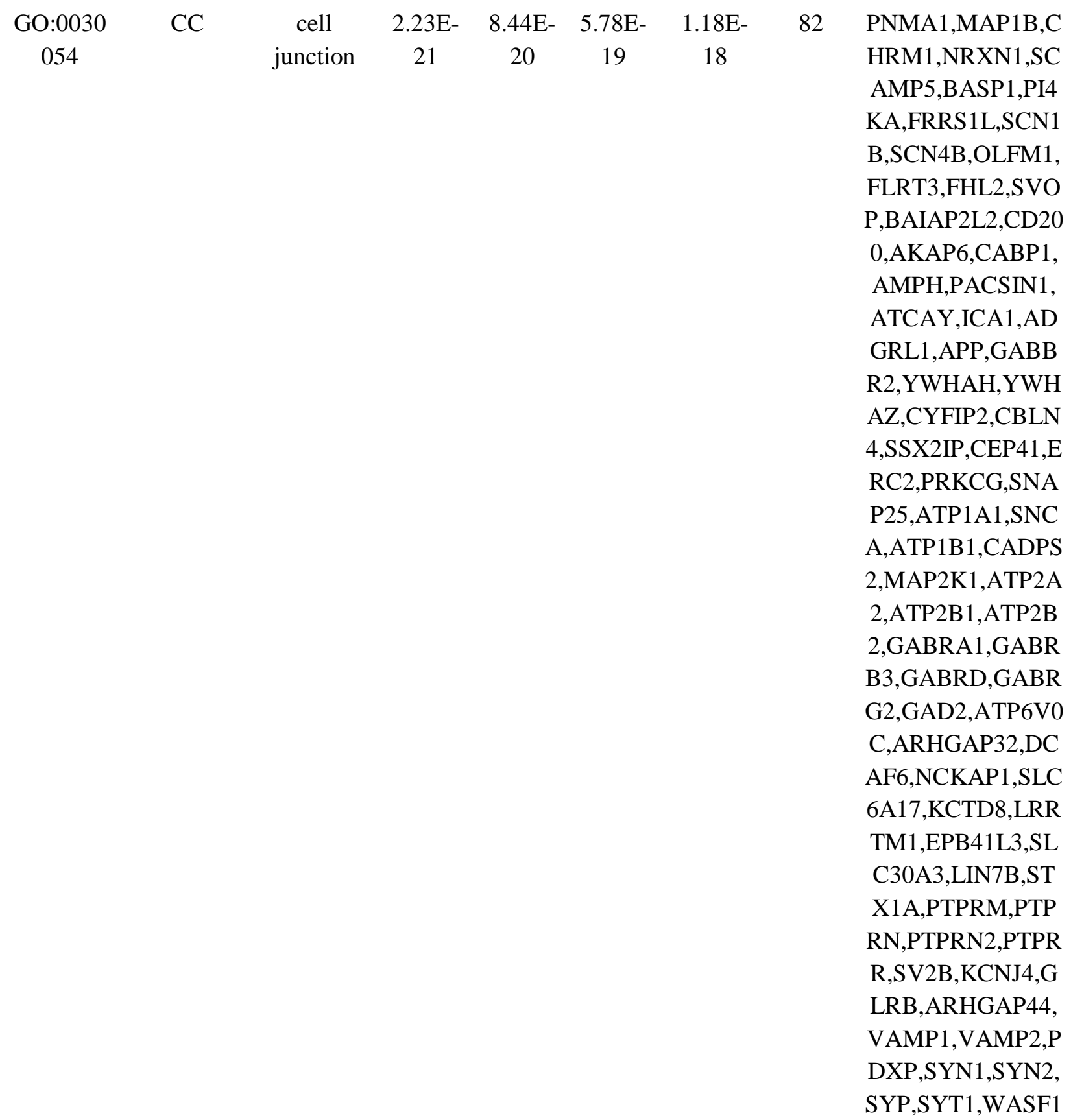


medRxiv preprint doi: https://doi.org/10.1101/2020.12.21.20248688; this version posted December 24, 2020. The copyright holder for this preprint (which was not certified by peer review) is the author/funder, who has granted medRxiv a license to display the preprint in perpetuity. All rights reserved. No reuse allowed without permission.

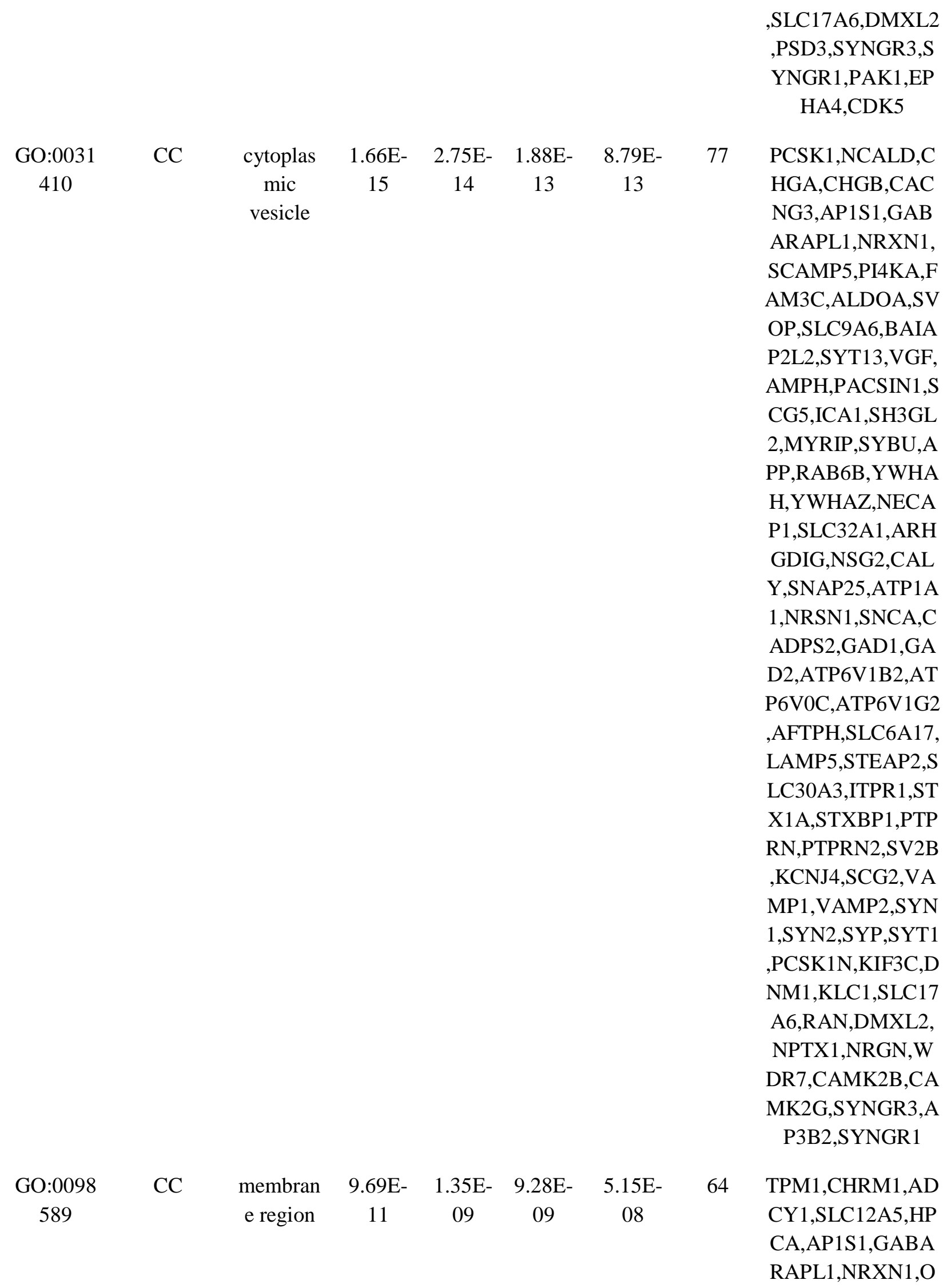


medRxiv preprint doi: https://doi.org/10.1101/2020.12.21.20248688; this version posted December 24, 2020. The copyright holder for this preprint (which was not certified by peer review) is the author/funder, who has granted medRxiv a license to display the preprint in perpetuity. All rights reserved. No reuse allowed without permission.

\begin{tabular}{|c|c|c|c|c|c|c|c|c|}
\hline $\begin{array}{c}\text { GO:0008 } \\
092\end{array}$ & MF & $\begin{array}{c}\text { cytoskele } \\
\text { tal } \\
\text { protein } \\
\text { binding }\end{array}$ & $\begin{array}{c}4.64 \mathrm{E}- \\
10\end{array}$ & $\begin{array}{c}2.58 \mathrm{E}- \\
07\end{array}$ & $\begin{array}{c}1.96 \mathrm{E}- \\
06\end{array}$ & $\begin{array}{c}5.15 \mathrm{E}- \\
07\end{array}$ & 50 & $\begin{array}{c}\text { TPM1,NCALD,M } \\
\text { AP1B,MAP2,MAP } \\
\text { 4,PFN2,HPCA,GA } \\
\text { BARAPL1,ALDO } \\
\text { A,BAIAP2L2,CAP } \\
\text { 2,PACSIN1,ATCA } \\
\text { Y,ENC1,MYRIP,G } \\
\text { AS7,SYBU,RAB6 } \\
\text { B,YWHAH,MICA } \\
\text { L2,PRKCE,SNAP2 } \\
\text { 5,ATP1A1,SNCB,S } \\
\text { NCA,ABLIM2,PR } \\
\text { NP,REEP1,EPB41 } \\
\text { L3,NEFM,NEFH,S } \\
\text { TX1A,PTPRN,VA } \\
\text { MP2,SYN1,KIF3C, } \\
\text { NME1,WASF1,CD }\end{array}$ \\
\hline
\end{tabular}


medRxiv preprint doi: https://doi.org/10.1101/2020.12.21.20248688; this version posted December 24, 2020. The copyright holder for this preprint (which was not certified by peer review) is the author/funder, who has granted medRxiv a license to display the preprint in perpetuity. All rights reserved. No reuse allowed without permission.

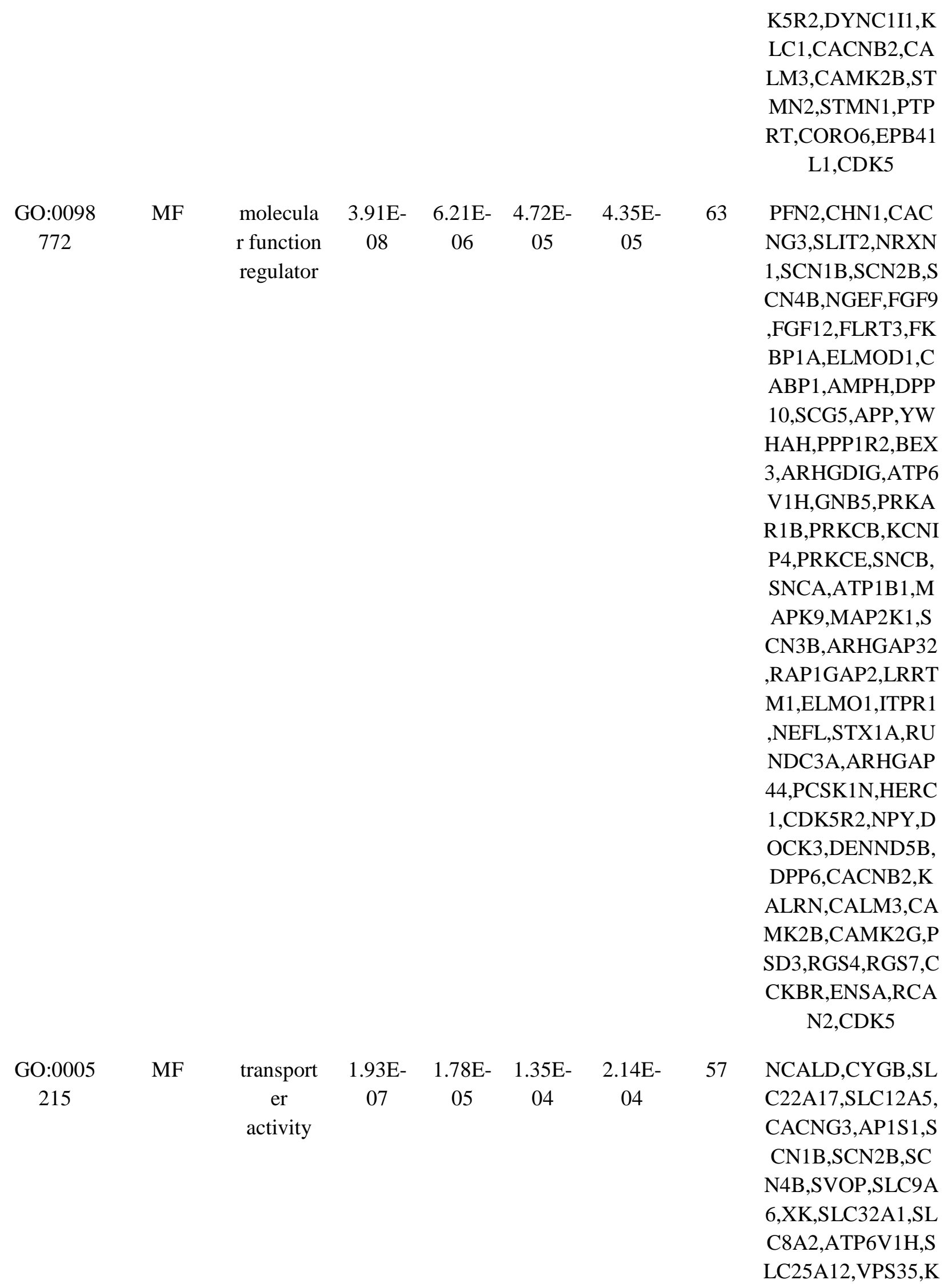


medRxiv preprint doi: https://doi.org/10.1101/2020.12.21.20248688; this version posted December 24, 2020. The copyright holder for this preprint (which was not certified by peer review) is the author/funder, who has granted medRxiv a license to display the preprint in perpetuity. All rights reserved. No reuse allowed without permission.

$\begin{array}{ccccccc}\text { GO:0005 } & \text { MF } & \begin{array}{c}\text { signaling } \\ \text { receptor }\end{array} & 3.70 \mathrm{E}- & 9.56 \mathrm{E}- & 7.25 \mathrm{E}- & 4.11 \mathrm{E}- \\ 102 & & 03 & 02 & 01 \\ & \text { binding } & & & & \end{array}$

CNIP4,SNAP25,A

TP1A1,ATP1B1,A

TP2A2,ATP2B1,A

TP2B2,ATP2B3,S

CN3B,GABRA1,G

ABRB3,GABRD,G

ABRG2,ATP6V1A

,ATP6V1B2,ATP6

V0C,ATP6V1E1,A

TP6V1G2,SLC6A1

7,CPLX1,TOMM2

0,STEAP2,SLC30

A3,ITPR1,SLC38A

1,SV2B,KCNJ4,GL

RB,KCNJ12,SYN1,

SYP,SLC17A6,DE

NND5B,GPM6A, R

AN,CACNB2,CAL

M3,TUSC3,SLC6A

15,AP3B2

ETS2,CHGB,CHN

1,ADCYAP1,SLIT

2,GABARAPL1,N

RXN1,UCHL1,NG

EF,FGF9,FAM3C,

FGF12,C1QTNF4,

FLRT3,FHL2,FKB

P1A,CD200,VGF, APP,YWHAH,BE

X3,NPTN,NSG1,C APRIN2,PRKCB,R

ASL11B,PRKCE,A TP2A2,ATP2B2, T

RIM37,REEP1,AD

AM23,SST,RUND

C3A,SCG2,SYT1,T

AC1,PCSK1N,GN

AO1,DNM1,GNG3

,NPY,RAN,CALM

3,NSF,CCK,CCKB

R,LINGO1,NCOA

7,RNF41,MRAP2,

ENSA,EPB41L1,E 
medRxiv preprint doi: https://doi.org/10.1101/2020.12.21.20248688; this version posted December 24, 2020. The copyright holder for this preprint (which was not certified by peer review) is the author/funder, who has granted medRxiv a license to display the preprint in perpetuity. All rights reserved. No reuse allowed without permission.

GO:0019 MF

899
8.69E- $1.89 \mathrm{E}-\quad 1.44 \mathrm{E}-\quad 9.65 \mathrm{E}-$

$\begin{array}{llll}04 & 02 & 01 & 01\end{array}$

enzyme

binding

$\begin{array}{ccccccc}\text { GO:0017 } & \text { MF } & \text { purine } & 3.22 \mathrm{E}- & 4.57 \mathrm{E}- & 3.47 \mathrm{E}- & 1.00 \mathrm{E}+0 \\ 076 & & \begin{array}{l}\text { nucleotid } \\ \text { e binding }\end{array} & 03 & 02 & 01 & 0\end{array}$

\section{PHA4,CDK5}

62 TSPYL1,PGAM1,S LC12A5,TUBA1B, HPCA,GABARAP L1,UBE2N,UCHL1 ,FBXL2,FKBP1A, AKAP6,MAP2K4,I ARS,MYRIP,APP, YWHAH,YWHAZ, PPP3CB,PPP3R1,C NTNAP2,PRKAR1 B,PRKCB,PRKCE, ATP1A1,SNCA,A TP1B1,MAPK9,M AP2K1,ATP2A2,T RIM37,ATP6V0C, ATP6V1E1,NCKA P1,MOAP1,SMYD 2,ITPR1,NEFH,NE LL1,NEFL,NELL2, STX1A,STXBP1,P TPRN,PTPRR,SY N1,SNU13,NME1, DNM1,WASF1,CA CNB2,DMXL2,KA LRN,CALM3,NSF, CAMK2B,LDB2,E GR1,NPTXR,PAK 1,RNF41,ENSA,C DK5

58 PDE1A,PFN2,CDK 14,ADCY1,TUBB2 A,CKMT1B,TUBA 1B,TUBB3,UBE2N ,PI4KA,MAP3K9, MAP2K4,SCG5,IA RS,RAB6B,ARF3, ASNS,AGK,PRKA R1B,PRKCB,RAS L11B,PRKCE,PRK CG,ATP1A1,ATP1 B1,MAPK9,MAP2 K1,ATP2A2,ATP2 
medRxiv preprint doi: https://doi.org/10.1101/2020.12.21.20248688; this version posted December 24, 2020. The copyright holder for this preprint (which was not certified by peer review) is the author/funder, who has granted medRxiv a license to display the preprint in perpetuity. All rights reserved. No reuse allowed without permission.

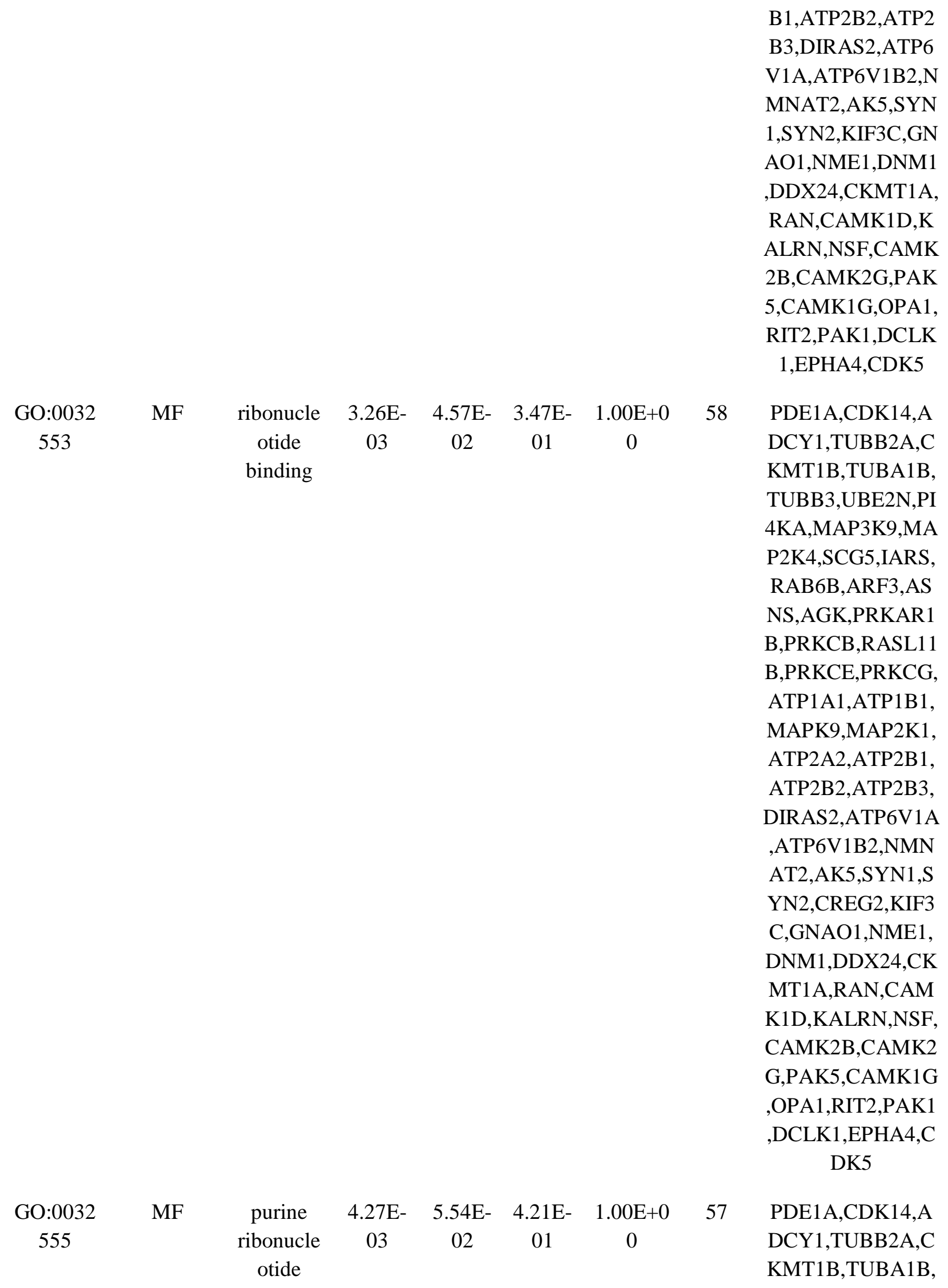


medRxiv preprint doi: https://doi.org/10.1101/2020.12.21.20248688; this version posted December 24, 2020. The copyright holder for this preprint (which was not certified by peer review) is the author/funder, who has granted medRxiv a license to display the preprint in perpetuity. All rights reserved. No reuse allowed without permission.

GO:0035 MF 639 binding

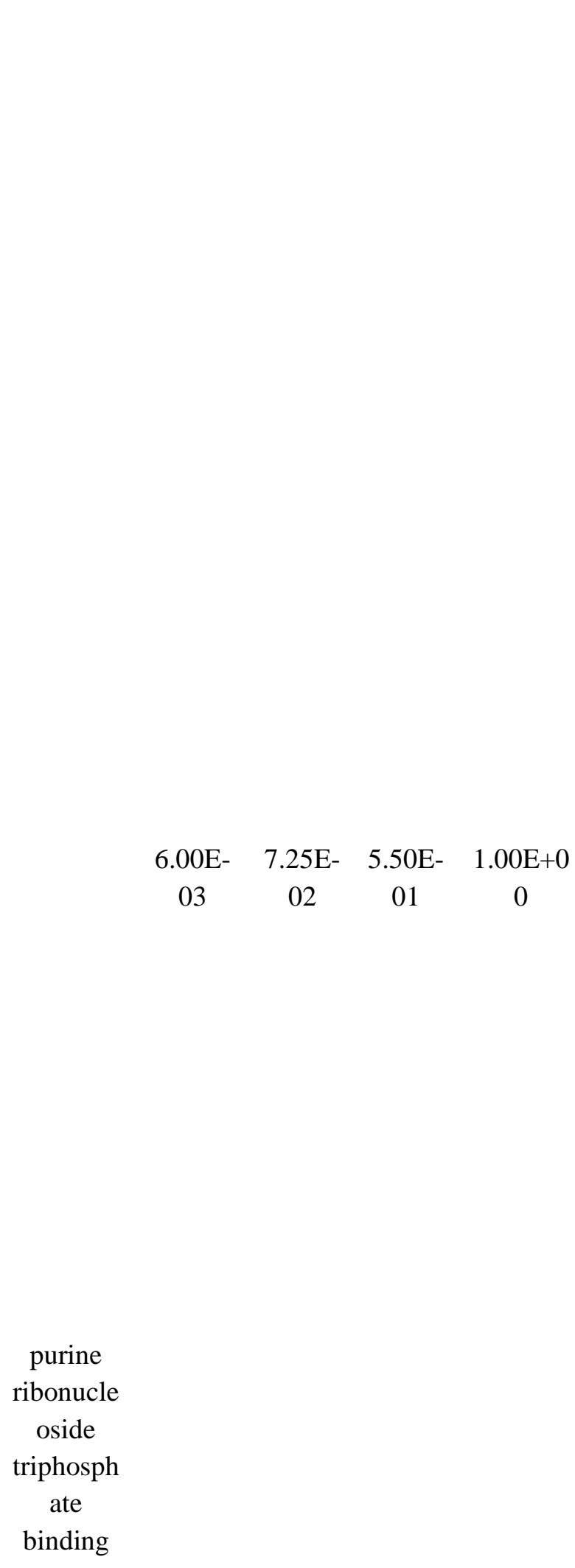

TUBB3,UBE2N,PI 4KA,MAP3K9,MA P2K4,SCG5,IARS, RAB6B,ARF3,AS NS,AGK,PRKAR1 B,PRKCB,RASL11 B,PRKCE,PRKCG, ATP1A1,ATP1B1, MAPK9,MAP2K1, ATP2A2,ATP2B1, ATP2B2,ATP2B3, DIRAS2,ATP6V1A ,ATP6V1B2,NMN AT2,AK5,SYN1,S YN2,KIF3C,GNA O1,NME1,DNM1, DDX24,CKMT1A, RAN,CAMK1D,K ALRN,NSF,CAMK 2B,CAMK2G,PAK 5,CAMK1G,OPA1, RIT2,PAK1,DCLK 1,EPHA4,CDK5

CDK14,ADCY1,T UBB2A,CKMT1B, TUBA1B,TUBB3, UBE2N,PI4KA,M AP3K9,MAP2K4,S CG5,IARS,RAB6B ,ARF3,ASNS,AGK ,PRKCB,RASL11B ,PRKCE,PRKCG,A TP1A1,ATP1B1,M APK9,MAP2K1,A TP2A2,ATP2B1,A TP2B2,ATP2B3,DI RAS2,ATP6V1A,A TP6V1B2,NMNAT 2,AK5,SYN1,SYN 2,KIF3C,GNAO1, NME1,DNM1,DD X24,CKMT1A,RA N,CAMK1D,KAL 
medRxiv preprint doi: https://doi.org/10.1101/2020.12.21.20248688; this version posted December 24, 2020. The copyright holder for this preprint (which was not certified by peer review) is the author/funder, who has granted medRxiv a license to display the preprint in perpetuity. All rights reserved. No reuse allowed without permission.

\begin{tabular}{|c|c|c|c|c|c|c|c|c|}
\hline & & & & & & & & $\begin{array}{l}\text { RN,NSF,CAMK2B } \\
\text {,CAMK2G,PAK5, } \\
\text { CAMK1G,OPA1,R } \\
\text { IT2,PAK1,DCLK1, } \\
\text { EPHA4,CDK5 }\end{array}$ \\
\hline $\begin{array}{c}\text { GO:0030 } \\
554\end{array}$ & $\mathrm{MF}$ & $\begin{array}{c}\text { adenyl } \\
\text { nucleotid } \\
\text { e binding }\end{array}$ & $\begin{array}{c}3.01 \mathrm{E}- \\
02\end{array}$ & $\begin{array}{c}1.88 \mathrm{E}- \\
01\end{array}$ & $\begin{array}{c}1.00 \mathrm{E} \\
+00\end{array}$ & $\begin{array}{c}1.00 \mathrm{E}+0 \\
0\end{array}$ & 44 & $\begin{array}{c}\text { PFN2,CDK14,ADC } \\
\text { Y1,CKMT1B,UBE } \\
\text { 2N,PI4KA,MAP3K } \\
\text { 9,MAP2K4,IARS, } \\
\text { ASNS,AGK,PRKA } \\
\text { R1B,PRKCB,PRK } \\
\text { CE,PRKCG,ATP1 } \\
\text { A1,ATP1B1,MAP } \\
\text { K9,MAP2K1,ATP2 } \\
\text { A2,ATP2B1,ATP2 } \\
\text { B2,ATP2B3,ATP6 } \\
\text { V1A,ATP6V1B2,N } \\
\text { MNAT2,AK5,SYN } \\
\text { 1,SYN2,KIF3C,N } \\
\text { ME1,DDX24,CKM } \\
\text { T1A,CAMK1D,KA } \\
\text { LRN,NSF,CAMK2 } \\
\text { B,CAMK2G,PAK5 } \\
\text {,CAMK1G,PAK1, } \\
\text { DCLK1,EPHA4,C } \\
\text { DK5 }\end{array}$ \\
\hline $\begin{array}{c}\text { GO:0042 } \\
802\end{array}$ & $\mathrm{MF}$ & $\begin{array}{c}\text { identical } \\
\text { protein } \\
\text { binding }\end{array}$ & $\begin{array}{c}4.09 \mathrm{E}- \\
02\end{array}$ & $\begin{array}{c}2.14 \mathrm{E}- \\
01\end{array}$ & $\begin{array}{c}1.00 \mathrm{E} \\
+00\end{array}$ & $\begin{array}{c}1.00 \mathrm{E}+0 \\
0\end{array}$ & 39 & $\begin{array}{c}\text { ACOT7,BCL11A, } \\
\text { ADARB1,SLIT2,H } \\
\text { PRT1,MAP3K9,FL } \\
\text { RT3,FHL2,ALDO } \\
\text { A,FKBP1A,CD200, } \\
\text { SH3GL2,APP,ZNF } \\
\text { 365,YWHAZ,CRY } \\
\text { M,ASNS,SNCA,T } \\
\text { RIM37,PRNP,NEL } \\
\text { L1,NEFL,NELL2,S } \\
\text { TXBP1,PTPRM,B } \\
\text { HLHE22,PVALB, } \\
\text { VAMP2,SYP,SYT } \\
\text { 1,NME1,DNM1,G } \\
\text { OT1,CAMK2B,CA } \\
\text { MK2G,OAT,MRA }\end{array}$ \\
\hline
\end{tabular}


medRxiv preprint doi: https://doi.org/10.1101/2020.12.21.20248688; this version posted December 24, 2020. The copyright holder for this preprint (which was not certified by peer review) is the author/funder, who has granted medRxiv a license to display the preprint in perpetuity.

All rights reserved. No reuse allowed without permission.

P2,ENO2,EPHA4

Table 5 The enriched GO terms of the down regulated differentially expressed genes

\begin{tabular}{|c|c|c|c|c|c|c|c|c|}
\hline GO ID & $\begin{array}{c}\text { CATEGO } \\
\text { RY }\end{array}$ & $\begin{array}{c}\text { GO } \\
\text { Name }\end{array}$ & $\begin{array}{c}\mathbf{P} \\
\text { Value }\end{array}$ & $\begin{array}{l}\text { FDR } \\
\text { B\&H }\end{array}$ & $\begin{array}{l}\text { FDR } \\
\text { B\&Y }\end{array}$ & $\begin{array}{c}\text { Bonferr } \\
\text { oni }\end{array}$ & $\begin{array}{c}\text { Gene } \\
\text { Cou } \\
\text { nt }\end{array}$ & Gene \\
\hline $\begin{array}{c}\text { GO:0002 } \\
682\end{array}$ & $\mathrm{BP}$ & $\begin{array}{l}\text { regulation } \\
\text { of } \\
\text { immune } \\
\text { system } \\
\text { process }\end{array}$ & $\begin{array}{c}1.44 \mathrm{E}- \\
20\end{array}$ & $\begin{array}{c}7.89 \mathrm{E}- \\
17\end{array}$ & $\begin{array}{c}7.25 \mathrm{E}- \\
16\end{array}$ & $\begin{array}{c}\text { 7.89E- } \\
17\end{array}$ & 94 & $\begin{array}{c}\text { CDKN1A,CMTM } \\
\text { 3,CEBPB,HLA- } \\
\text { DMA,HLA- } \\
\text { DMB,HLA- } \\
\text { DOA,HLA- } \\
\text { DPA1,HLA- } \\
\text { DQA1,PDK4,CD3 } \\
\text { 00A,HLA- } \\
\text { DRA,VSIG4,RFT } \\
\text { N2,HMOX1,LY96 } \\
\text {,VAMP3,TYROB } \\
\text { P,FCER1G,FCGR } \\
\text { 1A,SASH3,FCGR } \\
\text { T,AIF1,FGFR3,F } \\
\text { GR,MERTK,HSP } \\
\text { A1A,CHST3,VEG } \\
\text { FB,EZR,WAS,LA } \\
\text { T2,RGCC,SLC1A } \\
\text { 3,MSN,IFNGR1,S } \\
\text { LC7A2,SLC11A1, } \\
\text { CSF1R,CSF3R,CS } \\
\text { K,PYCARD,VAM } \\
\text { P8,SCIN,CTSH,G } \\
\text { PRC5B,CYBA,HT } \\
\text { RA1,MYOM1,TN } \\
\text { FSF14,RASSF2,M } \\
\text { YO10,AXL,BCL6 } \\
\text {,ITGB1,ITGB2,LI } \\
\text { LRB1,VSIR,HCS } \\
\text { T,STAT5A,ITPK } \\
\text { B,PTPN6,BST2,M } \\
\text { APKAPK3,SYK,C } \\
\text { 1QA,C1QB,C1QC } \\
\text {,ADAM17,UNC93 }\end{array}$ \\
\hline
\end{tabular}


medRxiv preprint doi: https://doi.org/10.1101/2020.12.21.20248688; this version posted December 24, 2020. The copyright holder for this preprint (which was not certified by peer review) is the author/funder, who has granted medRxiv a license to display the preprint in perpetuity. All rights reserved. No reuse allowed without permission.

\begin{tabular}{|c|c|c|c|c|c|c|c|c|}
\hline $\begin{array}{c}\text { GO:0001 } \\
775\end{array}$ & $\mathrm{BP}$ & $\begin{array}{c}\text { cell } \\
\text { activation }\end{array}$ & $\begin{array}{c}1.31 \mathrm{E}- \\
19\end{array}$ & $\begin{array}{c}3.58 \mathrm{E}- \\
16\end{array}$ & $\begin{array}{c}3.29 \mathrm{E}- \\
15\end{array}$ & $\begin{array}{c}7.17 \mathrm{E}- \\
16\end{array}$ & 73 & $\begin{array}{c}\text { CDKN1A,CEBPB } \\
\text {,HLA-DMA,HLA- } \\
\text { DMB,HLA- } \\
\text { DOA,HLA- } \\
\text { DPA1,HLA- } \\
\text { DQA1,CD300A,H } \\
\text { LA- } \\
\text { DRA,VSIG4,HM } \\
\text { OX1,VAMP3,FZ } \\
\text { D9,TYROBP,AD } \\
\text { RB2,NDRG1,FCE } \\
\text { R1G,SASH3,CLI } \\
\text { C1,AIF1,FGR,ME } \\
\text { RTK,PLEK,HSPB } \\
\text { 1,APBB1IP,WAS, } \\
\text { LAT2,RGCC,APO } \\
\text { E,MYL12A,PDPN } \\
\text {,RHOG,SLC7A2, } \\
\text { SLC11A1,CSK,P } \\
\text { YCARD,CSRP1,V } \\
\text { AMP8,MEGF10,T } \\
\text { NFSF14,AXL,BC } \\
\text { L6,ITGAX,ITGB1 } \\
\text {,ITGB2,LILRB1, } \\
\text { VSIR,STAT5A,IT } \\
\text { PKB,PTPN6,BST } \\
\text { 2,SYK,GNA12,A } \\
\text { DAM17,RAC2,FE } \\
\text { RMT3,DOCK2,LI } \\
\text { LRB3,GPER1,LC } \\
\text { P1,LGALS9,HAV } \\
\text { CR2,EBI3,TIMP1, }\end{array}$ \\
\hline
\end{tabular}


medRxiv preprint doi: https://doi.org/10.1101/2020.12.21.20248688; this version posted December 24, 2020. The copyright holder for this preprint (which was not certified by peer review) is the author/funder, who has granted medRxiv a license to display the preprint in perpetuity. All rights reserved. No reuse allowed without permission.

GO:0006 952

\section{BP}

defense
response

2.10

19

$3.82 \mathrm{E}-\quad 3.51 \mathrm{E}-$ 16
DCLRE1C,CD86, CD37,CD44,CD74 ,CD151,LYL1,LY

N,CMTM7

$1.15 \mathrm{E}-$ 15

\section{SERPINA3,CEBP} B,HLADPA1,HLADQA1,PDK4,CLE C5A,CD300A,HL A-

DRA,VSIG4,HM OX1,LY96,CD163 ,VAMP3,TYROB P,ADRB2,FCER1 G,FCGR1A,IFIT M3,AIF1,FGR,SE C61A1,HSPA1A, LY86,ALOX5,AL OX5AP,IL17RB,C HST3,DDIT4, WA S,IRF8,IFITM2,A POE,SP110,AQP4 ,SLC1A3,IFNGR1 ,SLC7A2,MT2A,S LC11A1,CSF1R,C SF3R,CSK,PRCP, PYCARD,XAF1, VAMP8,GPRC5B, CYBA,HTRA1,M YOM1,SPP1,AXL ,GBP2,DBI,BCL6, ITGAX,ITGB1,IT GB2,LILRB1,STA T5A,STAB1,GJA 1,PTPN6,BST2,M APKAPK3,SYK,C 1QA,C1QB,C1QC ,ADAM17,UNC93 B1,TAP1,DOCK2, PLA2G7,LILRB3, RARRES2,GPER 1,NTRK2,GPX1, OAS2,CXCL16,N 
medRxiv preprint doi: https://doi.org/10.1101/2020.12.21.20248688; this version posted December 24, 2020. The copyright holder for this preprint (which was not certified by peer review) is the author/funder, who has granted medRxiv a license to display the preprint in perpetuity. All rights reserved. No reuse allowed without permission.

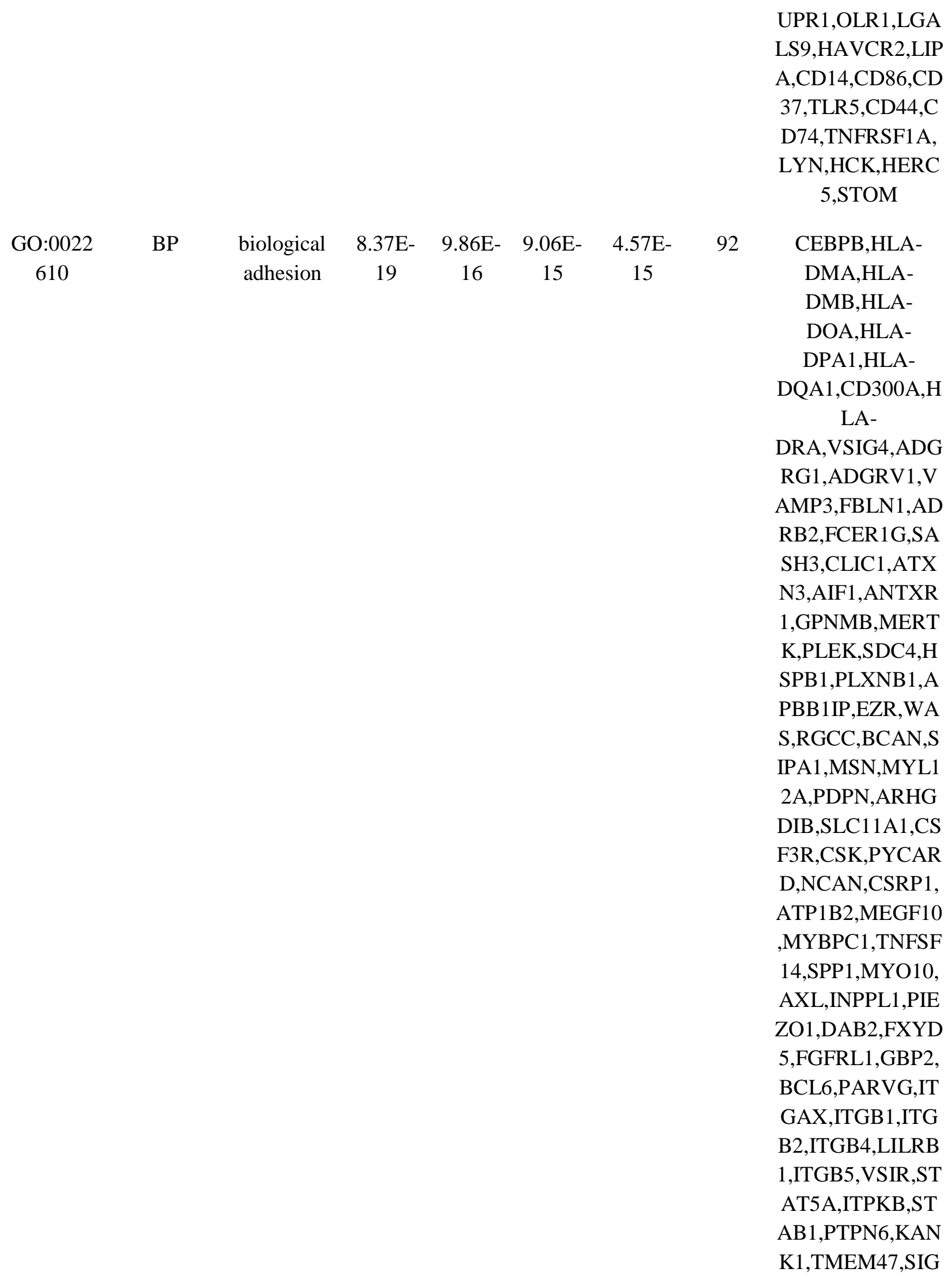


medRxiv preprint doi: https://doi.org/10.1101/2020.12.21.20248688; this version posted December 24, 2020. The copyright holder for this preprint (which was not certified by peer review) is the author/funder, who has granted medRxiv a license to display the preprint in perpetuity. All rights reserved. No reuse allowed without permission.

\begin{tabular}{|c|c|c|c|c|c|c|c|c|}
\hline $\begin{array}{c}\text { GO:0006 } \\
955\end{array}$ & $\mathrm{BP}$ & $\begin{array}{l}\text { immune } \\
\text { response }\end{array}$ & $\begin{array}{c}9.02 \mathrm{E}- \\
19\end{array}$ & $\begin{array}{c}9.86 \mathrm{E}- \\
16\end{array}$ & $\begin{array}{c}9.06 \mathrm{E}- \\
15\end{array}$ & $\begin{array}{c}4.93 \mathrm{E}- \\
15\end{array}$ & 93 & $\begin{array}{c}\text { CMTM3,CEBPB, } \\
\text { HLA-DMA,HLA- } \\
\text { DMB,HLA- } \\
\text { DOA,HLA- } \\
\text { DPA1,HLA- } \\
\text { DQA1,CLEC5A,C } \\
\text { D300A,HLA- } \\
\text { DRA,CTSC,VSIG } \\
\text { 4,RFTN2,HMOX1 } \\
\text {,LY96,VAMP3,T } \\
\text { YROBP,FCER1G, } \\
\text { FCGR1A,SASH3, } \\
\text { FCGRT,IFITM3,A } \\
\text { IF1,FGR,SEC61A } \\
\text { 1,HSPA1A,LY86, } \\
\text { APBB1IP,EZR,W } \\
\text { AS,LAT2,RGCC,I } \\
\text { RF8,IFITM2,SP11 } \\
\text { 0,AQP4,IFNGR1, } \\
\text { MT2A,SLC11A1, } \\
\text { CSF1R,CSK,PYC } \\
\text { ARD,XAF1,VAM } \\
\text { P8,CTSH,GPRC5 } \\
\text { B,CYBA,TNFSF1 } \\
\text { 4,MYO10,AXL,G } \\
\text { BP2,BCL6,ITGB1 } \\
\text {,ITGB2,LILRB1, } \\
\text { VSIR,HCST,STA } \\
\text { T5A,PTPN6,BST2 } \\
\text {,MAPKAPK3,SY } \\
\text { K,C1QA,C1QB,C } \\
\text { 1QC,ADAM17,U } \\
\text { NC93B1,TAP1,R }\end{array}$ \\
\hline
\end{tabular}


medRxiv preprint doi: https://doi.org/10.1101/2020.12.21.20248688; this version posted December 24, 2020. The copyright holder for this preprint (which was not certified by peer review) is the author/funder, who has granted medRxiv a license to display the preprint in perpetuity. All rights reserved. No reuse allowed without permission.

\begin{tabular}{|c|c|c|c|c|c|c|c|c|}
\hline $\begin{array}{c}\text { GO:0007 } \\
155\end{array}$ & BP & $\begin{array}{c}\text { cell } \\
\text { adhesion }\end{array}$ & $\begin{array}{c}1.67 \mathrm{E}- \\
18\end{array}$ & $\begin{array}{c}1.52 \mathrm{E}- \\
15\end{array}$ & $\begin{array}{c}1.40 \mathrm{E}- \\
14\end{array}$ & $\begin{array}{c}9.11 \mathrm{E}- \\
15\end{array}$ & 91 & $\begin{array}{c}\text { CEBPB,HLA- } \\
\text { DMA,HLA- } \\
\text { DMB,HLA- } \\
\text { DOA,HLA- } \\
\text { DPA1,HLA- } \\
\text { DQA1,CD300A,H } \\
\text { LA- } \\
\text { DRA,VSIG4,ADG } \\
\text { RG1,ADGRV1,V } \\
\text { AMP3,FBLN1,AD } \\
\text { RB2,FCER1G,SA } \\
\text { SH3,CLIC1,ATX } \\
\text { N3,AIF1,ANTXR } \\
\text { 1,GPNMB,MERT } \\
\text { K,PLEK,SDC4,H } \\
\text { SPB1,PLXNB1,A } \\
\text { PBB1IP,EZR,WA } \\
\text { S,RGCC,BCAN,S } \\
\text { IPA1,MSN,MYL1 } \\
\text { 2A,PDPN,ARHG } \\
\text { DIB,SLC11A1,CS } \\
\text { F3R,CSK,PYCAR } \\
\text { D,NCAN,CSRP1, } \\
\text { ATP1B2,MEGF10 } \\
\text {,MYBPC1,TNFSF } \\
\text { 14,SPP1,MYO10, } \\
\text { AXL,INPPL1,PIE } \\
\text { ZO1,DAB2,FXYD } \\
\text { 5,FGFRL1,BCL6, } \\
\text { PARVG,ITGAX,I } \\
\text { TGB1,ITGB2,ITG } \\
\text { B4,LILRB1,ITGB }\end{array}$ \\
\hline
\end{tabular}


medRxiv preprint doi: https://doi.org/10.1101/2020.12.21.20248688; this version posted December 24, 2020. The copyright holder for this preprint (which was not certified by peer review) is the author/funder, who has granted medRxiv a license to display the preprint in perpetuity. All rights reserved. No reuse allowed without permission.

GO:0040

011
BP locomotio

$\mathrm{n}$

$\begin{array}{cccc}1.94 \mathrm{E}- & 1.51 \mathrm{E}- & 1.39 \mathrm{E}- & 1.06 \mathrm{E}- \\ 17 & 14 & 13 & 13\end{array}$
5,VSIR,STAT5A,I

TPKB,STAB1,PT

PN6,KANK1,TM

EM47,SIGLEC10,

SYK,NINJ1,ADA

M17,FERMT2,RA

C2,FERMT3,DOC

K2,DDR1,DNAJB

6,LCP1,OLR1,LG

ALS9,HAVCR2,E

BI3,CD86,LPP,C

D44,CD74,CD151

,LYN,HCK

CMTM3,SYNJ2B

P,LEMD3,CLEC5

A,CD300A,ADGR

G1,HMOX1,SOX

8,FBLN1,SLC9A3

R1,FCER1G,IFIT

M3,RAPGEF3,SR

GAP1,AIF1,FOX

O4,FGR,MERTK,

HSPA1A,PLTP,S

DC4,HSPB1,PLX

NB1,TNS3,VEGF

B,EZR,DDIT4,W

AS,SGK1,RGCC,

ASAP3,IFITM2,A

POE,MSN,RHOC,

PDPN,RHOG,AR

HGDIB,CSF1R,C

SF3R,CSK,PRCP, PYCARD,VAMP

8,ATP1B2,CTSH,

PALLD,TNFSF14

,SPP1,MYO10,A

XL,SPTBN1,DAB

2,ITGAX,ITGB1,I

TGB2,ITGB4,ITG

B5,STAT5A,GJA

1,PTPN6,KANK1, BST2,TSPO,SYK, GNA12,ADAM17 
medRxiv preprint doi: https://doi.org/10.1101/2020.12.21.20248688; this version posted December 24, 2020. The copyright holder for this preprint (which was not certified by peer review) is the author/funder, who has granted medRxiv a license to display the preprint in perpetuity. All rights reserved. No reuse allowed without permission.

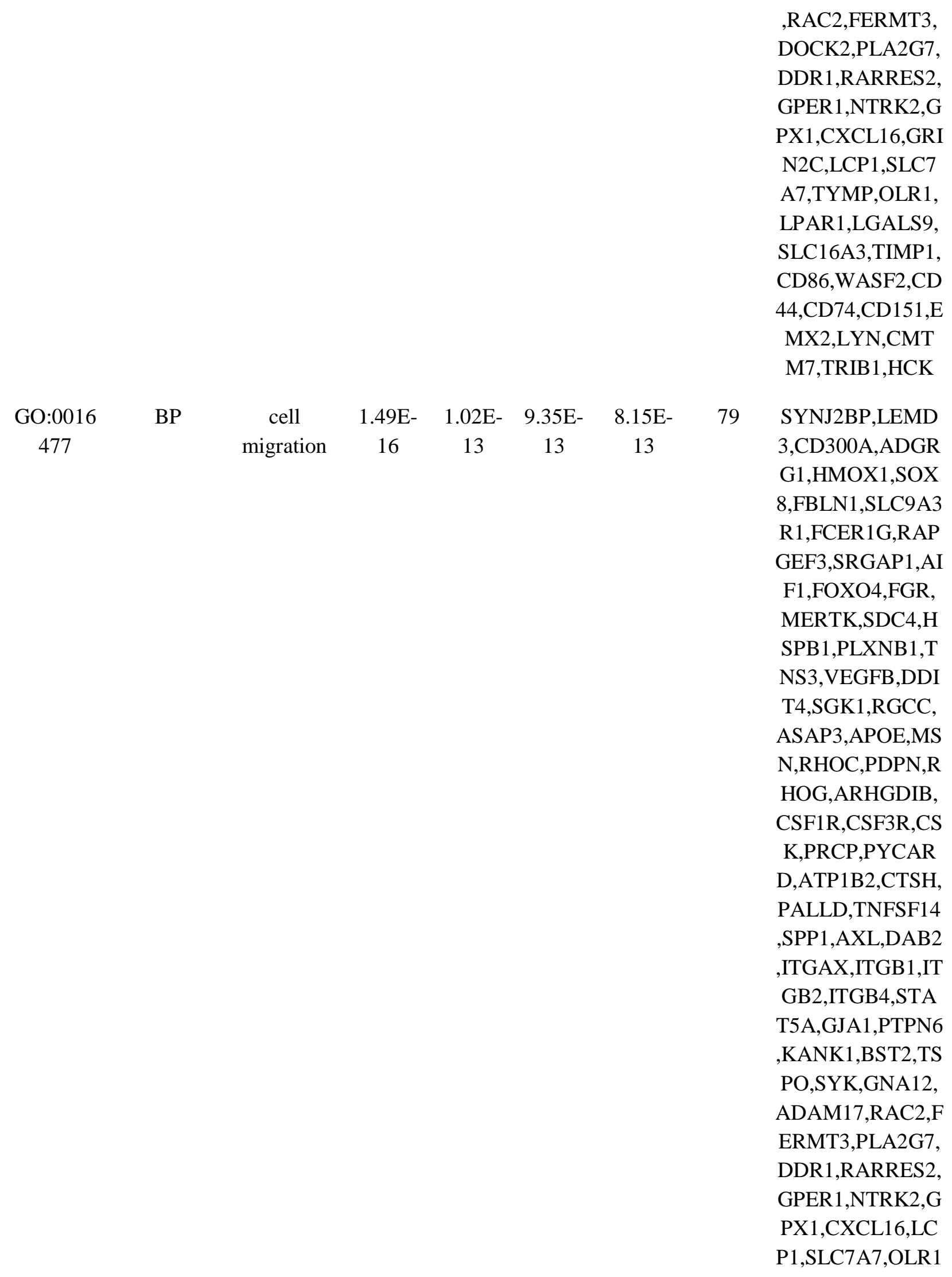


medRxiv preprint doi: https://doi.org/10.1101/2020.12.21.20248688; this version posted December 24, 2020. The copyright holder for this preprint (which was not certified by peer review) is the author/funder, who has granted medRxiv a license to display the preprint in perpetuity. All rights reserved. No reuse allowed without permission.

\begin{tabular}{|c|c|c|c|c|c|c|c|c|}
\hline $\begin{array}{c}\text { GO:0051 } \\
674\end{array}$ & $\mathrm{BP}$ & $\begin{array}{c}\text { localizatio } \\
\mathrm{n} \text { of cell }\end{array}$ & $\begin{array}{c}9.28 \mathrm{E}- \\
16\end{array}$ & $\begin{array}{c}4.23 \mathrm{E}- \\
13\end{array}$ & $\begin{array}{c}3.88 \mathrm{E}- \\
12\end{array}$ & $\begin{array}{c}5.07 \mathrm{E}- \\
12\end{array}$ & 82 & $\begin{array}{c}\text { SYNJ2BP,LEMD } \\
\text { 3,CD300A,ADGR } \\
\text { G1,HMOX1,SOX } \\
\text { 8,FBLN1,SLC9A3 } \\
\text { R1,FCER1G,RAP } \\
\text { GEF3,SRGAP1,AI } \\
\text { F1,FOXO4,FGR, } \\
\text { MERTK,PLTP,SD } \\
\text { C4,HSPB1,PLXN } \\
\text { B1,TNS3,VEGFB, } \\
\text { DDIT4,WAS,SGK } \\
\text { 1,RGCC,ASAP3, } \\
\text { APOE,MSN,RHO } \\
\text { C,PDPN,RHOG,A } \\
\text { RHGDIB,CSF1R, } \\
\text { CSF3R,CSK,PRC } \\
\text { P,PYCARD,ATP1 } \\
\text { B2,CTSH,PALLD } \\
\text {,TNFSF14,SPP1, } \\
\text { MYO10,AXL,DA } \\
\text { B2,ITGAX,ITGB1 } \\
\text {,ITGB2,ITGB4,ST } \\
\text { AT5A,GJA1,PTP } \\
\text { N6,KANK1,BST2 } \\
\text {,TSPO,SYK,GNA } \\
\text { 12,ADAM17,RAC } \\
\text { 2,FERMT3,PLA2 } \\
\text { G7,DDR1,RARRE } \\
\text { S2,GPER1,NTRK } \\
\text { 2,GPX1,CXCL16, } \\
\text { LCP1,SLC7A7,O } \\
\text { LR1,LPAR1,LGA } \\
\text { LS9,SLC16A3,TI } \\
\text { MP1,WASF2,CD4 } \\
\text { 4,CD74,CD151,E } \\
\text { MX2,LYN,TRIB1 } \\
\text {,HCK }\end{array}$ \\
\hline
\end{tabular}


medRxiv preprint doi: https://doi.org/10.1101/2020.12.21.20248688; this version posted December 24, 2020. The copyright holder for this preprint (which was not certified by peer review) is the author/funder, who has granted medRxiv a license to display the preprint in perpetuity. All rights reserved. No reuse allowed without permission.

\begin{tabular}{|c|c|c|c|c|c|c|}
\hline $\begin{array}{c}\text { GO:0042 } \\
127\end{array}$ & $\mathrm{BP}$ & $\begin{array}{c}\text { regulation } \\
\text { of cell } \\
\text { proliferati } \\
\text { on }\end{array}$ & $\begin{array}{c}2.73 \mathrm{E}- \\
14\end{array}$ & $\begin{array}{c}9.95 \mathrm{E}- \\
12\end{array}$ & $\begin{array}{c}9.14 \mathrm{E}- \\
11\end{array}$ & $\begin{array}{c}1.49 \mathrm{E}- \\
10\end{array}$ \\
\hline
\end{tabular}

87 CDKN1A,CEBPA ,CEBPB,HLADMB,SYNJ2BP,H LA-DPA1,HLADQA1,CD300A,V SIG4,GPR37L1,A DGRG1,HMOX1, SOX8,NDRG2,FB LN1,SLC9A3R1, ADRB2,NDRG1,S ASH3,IFITM3,AI F1,FOXO4,PLCD 1,FGFR3,GPNMB ,FGR,PLXNB1,T NS3,SERTAD1,V EGFB,SGK1,RGC C,HIPK2,APOE, MSX1,RHOG,CS F1R,CSK,PYCAR D,AKR1C3,SCIN, CTSH,CYBA,HT RA1,INPPL1,DA B2,FGFRL1,CCN D1,BCL6,ITGB1, LILRB1,GFAP,PT H1R,VSIR,STAT5 A,STK3,GJA1,PT PN6,TSPO,SYK, ADAM17,RAC2, DDR1,RARRES3, GPER1,NTRK2,G PX1,TMEM119,S ELENON,NUPR1, LGALS9,TGFB3, HAVCR2,EBI3,TI MP1,NACC2,CD8 6,CD37,CD44,CD 74,TNFRSF1A,E MP3,LYN,TRIB1, HCK,HCLS1,HD $\mathrm{AC} 1$ GO:0098 $\mathrm{CC}$ membrane 2.39E- $\quad 1.25 \mathrm{E}-\quad 8.57 \mathrm{E}-$ $1.25 \mathrm{E}-$ 12
ABCA1,CYBRD1 ,PDE9A,DHRS3,S 
medRxiv preprint doi: https://doi.org/10.1101/2020.12.21.20248688; this version posted December 24, 2020. The copyright holder for this preprint (which was not certified by peer review) is the author/funder, who has granted medRxiv a license to display the preprint in perpetuity. All rights reserved. No reuse allowed without permission.

LCO2B1,RFTN2, HMOX1,ADGRV

1,VAMP3,FZD9,S

LC9A3R1,ADRB

2,FCER1G,FCGR

1A,RAPGEF3,AH

NAK,AIF1,ANTX

R1,PLCD1,FGR,P

LEK,HSPA1A,CN

GB1,SDC4,HSPB

1,EZR,LAT2,ITS

N1,AQP4,SLC1A

3,SLC2A5,MSN,C

RYAB,PDPN,NE

CAP2,CSK,VAM

P8,ATP1B2,MEG

F10,GPRC5B,CY

BA,MYO10,PIEZ

O1,SPTBN1,DAB

2,MFRP,ITGB1,I

TGB2,ITGB4,PT

H1 R,HEPH,GJA1,

KANK1,BST2,KC

NMA1, GNA12,A

DAM17,FERMT2,

DDR1,GPER1,NT

RK2,LAMP2,GRI

N2C,LCP1,SLC7

A7,OLR1,RHOQ, CD14,SYTL4,CD

44,LRP4,TNFRSF

1A,LYN,HCK,ST

$\mathrm{OM}$

\begin{tabular}{|c|c|c|c|c|c|c|c|c|}
\hline $\begin{array}{c}\text { GO:0009 } \\
986\end{array}$ & $\mathrm{CC}$ & $\begin{array}{c}\text { cell } \\
\text { surface }\end{array}$ & $\begin{array}{c}5.42 \mathrm{E}- \\
13\end{array}$ & $\begin{array}{c}1.42 \mathrm{E}- \\
10\end{array}$ & $\begin{array}{c}9.72 \mathrm{E}- \\
10\end{array}$ & $\begin{array}{c}2.84 \mathrm{E}- \\
10\end{array}$ & 56 & $\begin{array}{c}\text { ABCA1,HLA- } \\
\text { DMA,SYNJ2BP,H } \\
\text { LA-DPA1,HLA- } \\
\text { DQA1,CLEC5A,H } \\
\text { LA- } \\
\text { DRA,ADGRV1,V } \\
\text { AMP3,FZD9,TYR } \\
\text { OBP,FCER1G,FC } \\
\text { GR1A,IFITM3,A } \\
\text { NTXR1,FGFR3,S }\end{array}$ \\
\hline
\end{tabular}


medRxiv preprint doi: https://doi.org/10.1101/2020.12.21.20248688; this version posted December 24, 2020. The copyright holder for this preprint (which was not certified by peer review) is the author/funder, who has granted medRxiv a license to display the preprint in perpetuity. All rights reserved. No reuse allowed without permission.

\begin{tabular}{|c|c|c|c|c|c|c|c|c|}
\hline $\begin{array}{c}\text { GO:0005 } \\
764\end{array}$ & $\mathrm{CC}$ & lysosome & $\begin{array}{c}4.78 \mathrm{E}- \\
12\end{array}$ & $\begin{array}{c}6.27 \mathrm{E}- \\
10\end{array}$ & $\begin{array}{c}4.29 \mathrm{E}- \\
09\end{array}$ & $\begin{array}{c}2.51 \mathrm{E}- \\
09\end{array}$ & 41 & $\begin{array}{c}\text { HLA-DMA,HLA- } \\
\text { DMB,HLA- } \\
\text { DOA,HLA- } \\
\text { DPA1,HLA- } \\
\text { DQA1,HLA- } \\
\text { DRA,CTSC,SLC1 } \\
\text { 5A3,ADRB2,IFIT } \\
\text { M3,TPP1,AHNA } \\
\text { K,FGFR3,GPC5,S } \\
\text { DC4,LAT2,VASN } \\
\text {,NPC2,BCAN,AP } \\
\text { OE,PRDX6,SLC1 } \\
\text { 1A1,PRCP,NCAN } \\
\text {,RNASET2,VAM } \\
\text { P8,CTSH,DAB2, } \\
\text { GFAP,TMBIM1,B } \\
\text { GN,LAPTM5,GJA } \\
\text { 1,UNC93B1,HAP } \\
\text { 1,LAMP2,LIPA,C } \\
\text { D68,CD74,LYN,H } \\
\text { CK }\end{array}$ \\
\hline $\begin{array}{c}\text { GO:0005 } \\
773\end{array}$ & $\mathrm{CC}$ & vacuole & $\begin{array}{c}7.77 \mathrm{E}- \\
11\end{array}$ & $\begin{array}{c}8.15 \mathrm{E}- \\
09\end{array}$ & $\begin{array}{c}5.57 \mathrm{E}- \\
08\end{array}$ & $\begin{array}{c}4.07 \mathrm{E}- \\
08\end{array}$ & 64 & $\begin{array}{c}\text { HLA-DMA,HLA- } \\
\text { DMB,HLA- } \\
\text { DOA,HLA- } \\
\text { DPA1,HLA- }\end{array}$ \\
\hline
\end{tabular}


medRxiv preprint doi: https://doi.org/10.1101/2020.12.21.20248688; this version posted December 24, 2020. The copyright holder for this preprint (which was not certified by peer review) is the author/funder, who has granted medRxiv a license to display the preprint in perpetuity. All rights reserved. No reuse allowed without permission.

\begin{tabular}{|c|c|c|c|c|c|c|c|c|}
\hline $\begin{array}{c}\text { GO:0098 } \\
590\end{array}$ & $\mathrm{CC}$ & $\begin{array}{c}\text { plasma } \\
\text { membrane } \\
\text { region }\end{array}$ & $\begin{array}{c}1.41 \mathrm{E}- \\
09\end{array}$ & $\begin{array}{c}8.23 \mathrm{E}- \\
08\end{array}$ & $\begin{array}{c}5.63 \mathrm{E}- \\
07\end{array}$ & $\begin{array}{c}7.41 \mathrm{E}- \\
07\end{array}$ & 55 & $\begin{array}{c}\text { CYBRD1,PDE9A, } \\
\text { DHRS3,SLCO2B1 } \\
\text { „HMOX1,ADGRV } \\
\text { 1,VAMP3,FZD9,S } \\
\text { LC9A3R1,ADRB } \\
\text { 2,RAPGEF3,AIF1 } \\
\text {,ANTXR1,FGR,P } \\
\text { LEK,HSPA1A,CN } \\
\text { GB1,HSPB1,EZR, } \\
\text { AQP4,SLC1A3,S } \\
\text { LC2A5,MSN,CR } \\
\text { YAB,PDPN,VAM } \\
\text { P8,ATP1B2,MEG } \\
\text { F10,CYBA,MYO1 } \\
\text { 0,PIEZO1,SPTBN } \\
\text { 1,DAB2,MFRP,IT }\end{array}$ \\
\hline
\end{tabular}


medRxiv preprint doi: https://doi.org/10.1101/2020.12.21.20248688; this version posted December 24, 2020. The copyright holder for this preprint (which was not certified by peer review) is the author/funder, who has granted medRxiv a license to display the preprint in perpetuity. All rights reserved. No reuse allowed without permission.

\begin{tabular}{|c|c|c|c|c|c|c|}
\hline $\begin{array}{c}\text { GO:0031 } \\
226\end{array}$ & $\mathrm{CC}$ & $\begin{array}{c}\text { intrinsic } \\
\text { componen } \\
\text { t of } \\
\text { plasma } \\
\text { membrane }\end{array}$ & $\begin{array}{c}2.79 \mathrm{E}- \\
07\end{array}$ & $\begin{array}{c}6.63 \mathrm{E}- \\
06\end{array}$ & $\begin{array}{c}4.54 \mathrm{E}- \\
05\end{array}$ & $\begin{array}{c}1.46 \mathrm{E}- \\
04\end{array}$ \\
\hline
\end{tabular}

GB1,ITGB4,PTH1

R,HEPH,GJA1,K

ANK1,BST2,KCN

MA1,GNA12,AD

AM17,FERMT2,D

DR1,GPER1,NTR

K2,GRIN2C,LCP1

,SLC7A7,SYTL4,

CD44,LRP4,HCK
ABCA1,HLA-
DPA1,SLCO2B1,
HLA-
DQA1,CLEC5A,H
LA-

DRA,ADGRG1,L

Y96,CD163,TYR

OBP,ADRB2,FCE

R1G,APLNR,FGF

R3,GPC5,GPNMB

,MERTK,CNGB1,

SDC4,IL17RB,PL

XNB1,AQP4,SLC

2A5,SLC5A3,IFN

GR1,PDPN,SLC7

A2,SLC11A1,CSF

1R,CSF2RA,CSF3

R,ATP1B2,CYBA

,IL13RA1,VAMP

5,AXL,DAB2,FX

YD5,FGFRL1,IT

GAX,ITGB1,ITG

B2,ITGB4,ITGB5,

PTH1R,LAPTM5,

STAB1,GJA1,BS

T2,KCNMA1,AD

AM17,DDR1,LIL

RB3,GPER1,NTR

K2,GRIN2C,SLC7

A7,OLR1,LPAR1,

CD14,SLC16A3,G

YPC,CD37,TLR5,

CD44,CD74,CD15

1,TNFRSF1A,LY 
medRxiv preprint doi: https://doi.org/10.1101/2020.12.21.20248688; this version posted December 24, 2020. The copyright holder for this preprint (which was not certified by peer review) is the author/funder, who has granted medRxiv a license to display the preprint in perpetuity. All rights reserved. No reuse allowed without permission.

\begin{tabular}{|c|c|c|c|c|c|c|c|c|}
\hline & & & & & & & & N,STOM \\
\hline $\begin{array}{c}\text { GO:0005 } \\
887\end{array}$ & $\mathrm{CC}$ & $\begin{array}{c}\text { integral } \\
\text { componen } \\
t \text { of } \\
\text { plasma } \\
\text { membrane }\end{array}$ & $\begin{array}{c}3.12 \mathrm{E}- \\
07\end{array}$ & $\begin{array}{c}7.11 \mathrm{E}- \\
06\end{array}$ & $\begin{array}{c}4.87 \mathrm{E}- \\
05\end{array}$ & $\begin{array}{c}1.64 \mathrm{E}- \\
04\end{array}$ & 68 & $\begin{array}{c}\text { ABCA1,HLA- } \\
\text { DPA1,SLCO2B1, } \\
\text { HLA- } \\
\text { DQA1,CLEC5A,H } \\
\text { LA- } \\
\text { DRA,ADGRG1,C } \\
\text { D163,TYROBP,A } \\
\text { DRB2,FCER1G,A } \\
\text { PLNR,FGFR3,GP } \\
\text { C5,GPNMB,MER } \\
\text { TK,CNGB1,SDC4 } \\
\text {,IL17RB,PLXNB1 } \\
\text {,AQP4,SLC2A5,S } \\
\text { LC5A3,IFNGR1,P } \\
\text { DPN,SLC7A2,SL } \\
\text { C11A1,CSF1R,CS } \\
\text { F2RA,CSF3R,AT } \\
\text { P1B2,CYBA,IL13 } \\
\text { RA1,VAMP5,AX } \\
\text { L,DAB2,FXYD5, } \\
\text { FGFRL1,ITGAX,I } \\
\text { TGB1,ITGB2,ITG } \\
\text { B4,ITGB5,PTH1R } \\
\text {,LAPTM5,STAB1 } \\
\text {,GJA1,BST2,KCN } \\
\text { MA1,ADAM17,D } \\
\text { DR1,LILRB3,GPE } \\
\text { R1,NTRK2,GRIN } \\
\text { 2C,SLC7A7,OLR } \\
\text { 1,LPAR1,SLC16A } \\
\text { 3,GYPC,CD37,TL } \\
\text { R5,CD44,CD74,C } \\
\text { D151,TNFRSF1A, } \\
\text { LYN,STOM }\end{array}$ \\
\hline $\begin{array}{c}\text { GO:0030 } \\
054\end{array}$ & $\mathrm{CC}$ & $\begin{array}{c}\text { cell } \\
\text { junction }\end{array}$ & $\begin{array}{c}4.23 \mathrm{E}- \\
07\end{array}$ & $\begin{array}{c}9.24 \mathrm{E}- \\
06\end{array}$ & $\begin{array}{c}6.32 \mathrm{E}- \\
05\end{array}$ & $\begin{array}{c}2.22 \mathrm{E}- \\
04\end{array}$ & 54 & $\begin{array}{l}\text { ADD3,VAMP3,N } \\
\text { DRG1,AHNAK,F } \\
\text { GFR3,HSPA1A,S } \\
\text { DC4,CNN3,HSPB } \\
\text { 1,ARHGEF6,APB } \\
\text { B1IP,TNS3,EZR, } \\
\text { WAS,ITSN1,ASA } \\
\text { P3,AQP4,MSN,R }\end{array}$ \\
\hline
\end{tabular}


medRxiv preprint doi: https://doi.org/10.1101/2020.12.21.20248688; this version posted December 24, 2020. The copyright holder for this preprint (which was not certified by peer review) is the author/funder, who has granted medRxiv a license to display the preprint in perpetuity. All rights reserved. No reuse allowed without permission.

$\begin{array}{cccccccc}\text { GO:0031 } & \text { CC } & \text { cytoplasm } & 7.77 \mathrm{E}- & 1.62 \mathrm{E}- & 1.11 \mathrm{E}- & 4.07 \mathrm{E}- & 58 \\ 410 & & \text { ic vesicle } & 07 & 05 & 04 & 04 & \end{array}$

HOG,CSK,CSRP1

,PALLD,CYBA,V

AMP5,DAB2,FGF

RL1,CCND1,PAR

VG,ITGB1,ITGB4

,ITGB5,VSIR,GJ

A1,PTPN6,TMEM

47,GNA12,ADA

M17,FERMT2,RA

C2,FERMT3,GPE

R1,HAP1,DTNA,

GRIN2C,LCP1,H

AVCR2,FARP1,

WASF2,LPP,CD4

4,CD151,CGNL1, LYN,HCK

ic vesicte 07
SERPINA3,ABC
A1,HLA-
DPA1,HLA-
DQA1,HLA-

DRA,CTSC,CD16

3,VAMP3,FCGR1

A,IFITM3,TPP1,F GFR3,GPNMB,C

NGB1,VEGFB,IT

SN1,APOE,SIPA1

,PRDX6,MSN,AR

HGDIB,NECAP2,

SLC11A1,VAMP

8,ARRDC2,CTSH

,GPRC5B,CYBA, VAMP5,DAB2,F

GFRL1,GBP2,DB

I,ITGB1,ITGB2,I

TGB5,BGN,STAB

1,GJA1,ACSBG1, SYK,UNC93B1,R

AC2,RAB31,RAR

RES2,ATP6V0E1, GPER1,HAP1,LA MP2,LPAR1,TGF B3,RHOQ,TIMP1, SYTL4,CD74,HC 
medRxiv preprint doi: https://doi.org/10.1101/2020.12.21.20248688; this version posted December 24, 2020. The copyright holder for this preprint (which was not certified by peer review) is the author/funder, who has granted medRxiv a license to display the preprint in perpetuity. All rights reserved. No reuse allowed without permission.

\begin{tabular}{|c|c|c|c|c|c|c|c|c|}
\hline $\begin{array}{c}\text { GO:0005 } \\
615\end{array}$ & $\mathrm{CC}$ & $\begin{array}{c}\text { extracellul } \\
\text { ar space }\end{array}$ & $\begin{array}{c}6.31 \mathrm{E}- \\
06\end{array}$ & $\begin{array}{c}1.00 \mathrm{E}- \\
04\end{array}$ & $\begin{array}{c}6.85 \mathrm{E}- \\
04\end{array}$ & $\begin{array}{c}3.31 \mathrm{E}- \\
03\end{array}$ & 58 & $\begin{array}{c}\text { SERPINA3,CMT } \\
\text { M3,ABCA1,CTSC } \\
\text {,HMOX1,LY96,C } \\
\text { HI3L2,TST,FBLN } \\
\text { 1,SERPINB6,AEB } \\
\text { P1,CLIC1,GPC5, } \\
\text { MERTK,HSPA1A } \\
\text {,LY86,ALOX5,PL } \\
\text { TP,HSPB1,VEGF } \\
\text { B,EZR,COL20A1, } \\
\text { VASN,BCAN,AP } \\
\text { OC1,APOC2,APO } \\
\text { E,PRDX6,MSN,A } \\
\text { SIP,RNASET2,CT } \\
\text { SH,GPRC5B,HTR } \\
\text { A1,TNFSF14,SPP } \\
\text { 1,AXL,NDP,DBI, } \\
\text { ADA2,C1QA,C1Q } \\
\text { B,C1QC,PLA2G7, } \\
\text { DDR1,SELENBP } \\
\text { 1,CXCL16,LAMP } \\
\text { 2,LCP1,LGALS9, } \\
\text { TGFB3,CD14,EBI } \\
\text { 3,TIMP1,ANGPT } \\
\text { L4,TNFRSF1A,C } \\
\text { MTM7,STOM }\end{array}$ \\
\hline $\begin{array}{c}\text { GO:0019 } \\
899\end{array}$ & $\mathrm{MF}$ & $\begin{array}{l}\text { enzyme } \\
\text { binding }\end{array}$ & $\begin{array}{c}1.38 \mathrm{E}- \\
06\end{array}$ & $\begin{array}{c}1.67 \mathrm{E}- \\
03\end{array}$ & $\begin{array}{c}1.28 \mathrm{E}- \\
02\end{array}$ & $\begin{array}{c}1.67 \mathrm{E}- \\
03\end{array}$ & 74 & $\begin{array}{c}\text { CDKN1A,EPHX1, } \\
\text { ABCA1,CEBPA,C } \\
\text { EBPB,CARHSP1, } \\
\text { CD300A,CTSC,H } \\
\text { MOX1,ADD3,S10 } \\
\text { 0A1,SERPINB6,S } \\
\text { LC9A3R1,ADRB } \\
\text { 2,NDRG1,SRGAP } \\
\text { 1,ATXN3,FOXO4 } \\
\text {,FGR,PLEK,HSP } \\
\text { A1A,ALOX5AP,S } \\
\text { DC4,HSPB1,EZR, } \\
\text { WAS,WFS1,SGK } \\
\text { 1,RHOBTB3,RGC } \\
\text { C,NPC2,APOC2,P } \\
\text { RDX6,MSN,PPP1 }\end{array}$ \\
\hline
\end{tabular}


medRxiv preprint doi: https://doi.org/10.1101/2020.12.21.20248688; this version posted December 24, 2020. The copyright holder for this preprint (which was not certified by peer review) is the author/funder, who has granted medRxiv a license to display the preprint in perpetuity. All rights reserved. No reuse allowed without permission.

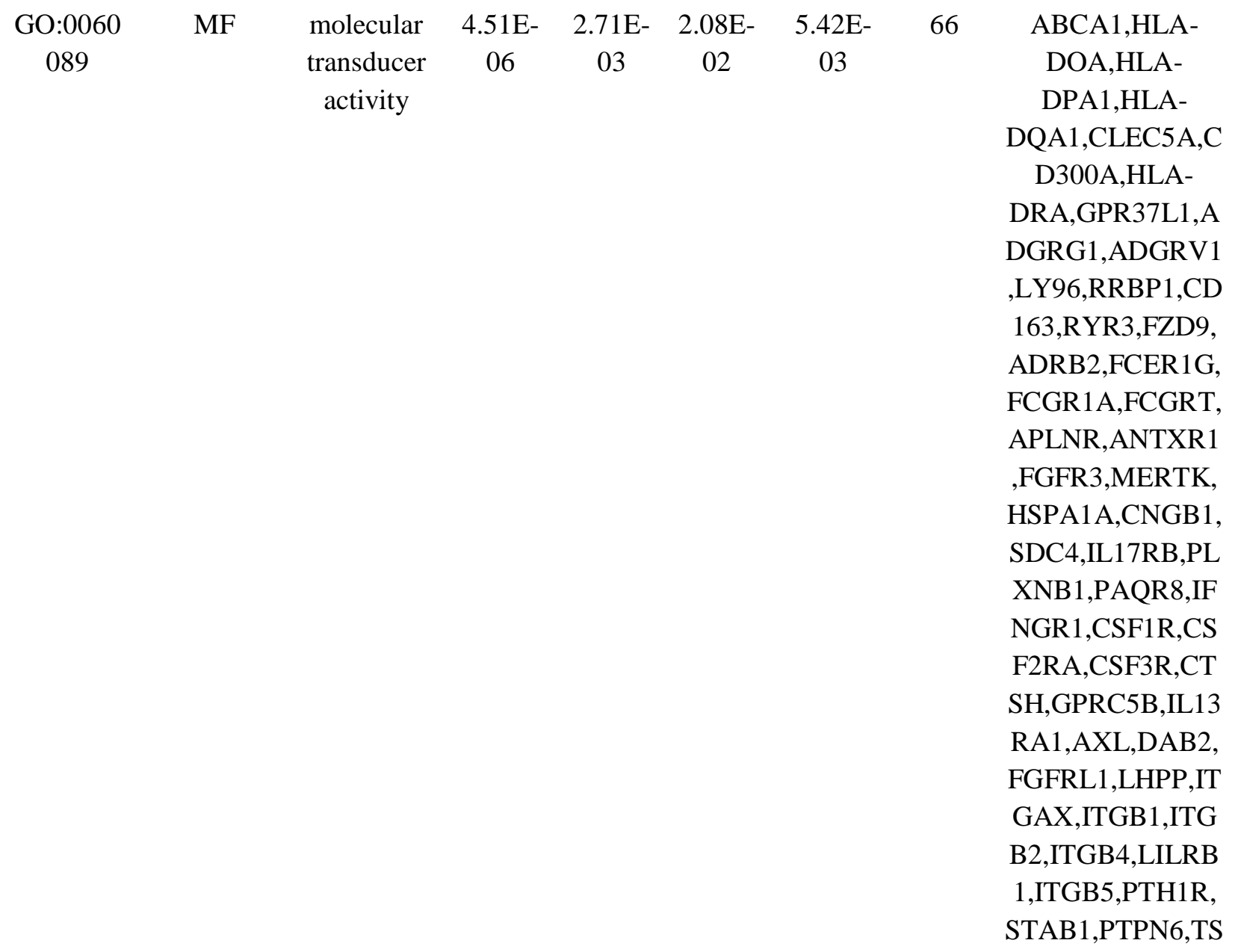


medRxiv preprint doi: https://doi.org/10.1101/2020.12.21.20248688; this version posted December 24, 2020. The copyright holder for this preprint (which was not certified by peer review) is the author/funder, who has granted medRxiv a license to display the preprint in perpetuity. All rights reserved. No reuse allowed without permission.

\begin{tabular}{|c|c|c|c|c|c|c|c|c|}
\hline $\begin{array}{c}\text { GO:0005 } \\
102\end{array}$ & $\mathrm{MF}$ & $\begin{array}{c}\text { signaling } \\
\text { receptor } \\
\text { binding }\end{array}$ & $\begin{array}{c}1.68 \mathrm{E}- \\
05\end{array}$ & $\begin{array}{c}3.63 \mathrm{E}- \\
03\end{array}$ & $\begin{array}{c}2.78 \mathrm{E}- \\
02\end{array}$ & $\begin{array}{c}2.03 \mathrm{E}- \\
02\end{array}$ & 61 & $\begin{array}{c}\text { CMTM3,ABCA1, } \\
\text { CEBPB,SYNJ2BP } \\
\text {,TYROBP,FBLN1 } \\
\text {,SLC9A3R1,ADR } \\
\text { B2,FCGR1A,PRM } \\
\text { T2,GPNMB,FGR, } \\
\text { HSPA1A,PLXNB } \\
\text { 1,VEGFB,APOE, } \\
\text { MSN,CSK,ASIP,P } \\
\text { YCARD,GPRC5B } \\
\text {,TNFSF14,SPP1,D } \\
\text { AB2,NDP,DBI,IT } \\
\text { GB1,ADA2,ITGB } \\
\text { 4,LILRB1,ITGB5, } \\
\text { GFAP,TMBIM1,H } \\
\text { CST,GJA1,PTPN6 } \\
\text {,SYK,GNA12,AD } \\
\text { AM17,UNC93B1, } \\
\text { TAP1,FERMT3,D } \\
\text { OCK2,RARRES2, } \\
\text { HAP1,NTRK2,CX } \\
\text { CL16,TYMP,TGF } \\
\text { B3,EBI3,TIMP1,S } \\
\text { YTL4,CD86,TLR } \\
\text { 5,CD44,LRP4,CD } \\
\text { 74,CD151,LYN,C } \\
\text { MTM7,HCK }\end{array}$ \\
\hline $\begin{array}{c}\text { GO:0042 } \\
802\end{array}$ & $\mathrm{MF}$ & $\begin{array}{c}\text { identical } \\
\text { protein } \\
\text { binding }\end{array}$ & $\begin{array}{c}1.74 \mathrm{E}- \\
05\end{array}$ & $\begin{array}{c}3.63 \mathrm{E}- \\
03\end{array}$ & $\begin{array}{c}2.78 \mathrm{E}- \\
02\end{array}$ & $\begin{array}{c}2.09 \mathrm{E}- \\
02\end{array}$ & 54 & $\begin{array}{c}\text { CEBPA,CEBPB,C } \\
\text { EBPD,CTSC,HM } \\
\text { OX1,PLEKHB1,S } \\
\text { 100A1,FZD9,TYR } \\
\text { OBP,FBLN1,ADR } \\
\text { B2,ATXN3,PRMT } \\
\text { 2,FOXO4,PLEK,P } \\
\text { LOD1,ALOX5AP, }\end{array}$ \\
\hline
\end{tabular}


medRxiv preprint doi: https://doi.org/10.1101/2020.12.21.20248688; this version posted December 24, 2020. The copyright holder for this preprint (which was not certified by peer review) is the author/funder, who has granted medRxiv a license to display the preprint in perpetuity. All rights reserved. No reuse allowed without permission.

$\begin{array}{cccccccc}\text { GO:0098 } & \text { MF } & \text { molecular } & 3.57 \mathrm{E}- & 5.41 \mathrm{E}- & 4.15 \mathrm{E}- & 4.29 \mathrm{E}- & 55 \\ 772 & & \text { function } & 05 & 03 & 02 & 02 & \\ & \text { regulator } & & & & \end{array}$

HSPB1,VEGFB WAS,S100A16,A POC2,BCAS1,AP OE,CRYAB,SLC1 1A1,CSF1R,CSK, PYCARD,ALDH4 A1,MAPK4,MYO M1,LHPP,NDP,A DA2,LILRB1,BST 2,NQO1,KCNMA 1,C1QB,TAP1,HS PB8,HAP1,NTRK 2,RENBP,LCP1,T GFB3,GSTM3,NA CC2,ANGPTL4,L RP4,CD74,TP53B $\mathrm{P} 2$,STOM

CDKN1A,SERPI NA3,CTSC,FBLN 1,SERPINB6,SLC 9A3R1,ADRB2,R APGEF3,SRGAP1 ,RIN2,FGFR3,AL OX5AP,HSPB1,A RHGEF6,PLXNB 1,PSD2,WAS,SG K1,ITSN1,RGCC, BAG3,ASAP3,AP OC1,APOC2,APO E,SIPA1,PPP1R3 C,ARHGDIB,CSF 2RA,PYCARD,V AMP8,ATP1B2,C TSH,GPRC5B,CY TH4,ADAP2,TNF SF14,SPTBN1,FX YD5,CCND1,BG

N,STK3,BST2,RA B3IL1,RAC2,DO CK2,DNAJB6,RE NBP,GRIN2C,RG S1,RGS10,TIMP1, FARP1,ANGPTL 
medRxiv preprint doi: https://doi.org/10.1101/2020.12.21.20248688; this version posted December 24, 2020. The copyright holder for this preprint (which was not certified by peer review) is the author/funder, who has granted medRxiv a license to display the preprint in perpetuity. All rights reserved. No reuse allowed without permission.

\begin{tabular}{|c|c|c|c|c|c|c|c|c|}
\hline & & & & & & & & 4,TRIB1 \\
\hline $\begin{array}{c}\text { GO:0038 } \\
023\end{array}$ & MF & $\begin{array}{l}\text { signaling } \\
\text { receptor } \\
\text { activity }\end{array}$ & $\begin{array}{c}7.68 \mathrm{E}- \\
05\end{array}$ & $\begin{array}{c}7.56 \mathrm{E}- \\
03\end{array}$ & $\begin{array}{c}5.80 \mathrm{E}- \\
02\end{array}$ & $\begin{array}{c}9.25 \mathrm{E}- \\
02\end{array}$ & 54 & $\begin{array}{c}\text { ABCA1,HLA- } \\
\text { DOA,HLA- } \\
\text { DPA1,HLA- } \\
\text { DQA1,CD300A,H } \\
\text { LA- } \\
\text { DRA,GPR37L1,A } \\
\text { DGRG1,ADGRV1 } \\
\text {,LY96,RYR3,FZD } \\
\text { 9,ADRB2,FCER1 } \\
\text { G,FCGR1A,FCGR } \\
\text { T,APLNR,ANTX } \\
\text { R1,FGFR3,MERT } \\
\text { K,SDC4,IL17RB, } \\
\text { PLXNB1,PAQR8, } \\
\text { IFNGR1,CSF1R,C } \\
\text { SF2RA,CSF3R,C } \\
\text { TSH,GPRC5B,IL1 } \\
\text { 3RA1,AXL,FGFR } \\
\text { L1,LHPP,ITGB1,I } \\
\text { TGB2,LILRB1,PT } \\
\text { H1R,PTPN6,TSP } \\
\text { O,DDR1,LILRB3, } \\
\text { LPAR5,GPER1,N } \\
\text { TRK2,GRIN2C,L } \\
\text { PAR1,CD14,EBI3 } \\
\text {,CD86,TLR5,CD4 } \\
\text { 4,CD74,TNFRSF1 } \\
\text { A }\end{array}$ \\
\hline $\begin{array}{c}\text { GO:0046 } \\
983\end{array}$ & $\mathrm{MF}$ & $\begin{array}{c}\text { protein } \\
\text { dimerizati } \\
\text { on activity }\end{array}$ & $\begin{array}{c}1.15 \mathrm{E}- \\
04\end{array}$ & $\begin{array}{c}9.22 \mathrm{E}- \\
03\end{array}$ & $\begin{array}{c}7.07 \mathrm{E}- \\
02\end{array}$ & $\begin{array}{c}1.38 \mathrm{E}- \\
01\end{array}$ & 50 & $\begin{array}{c}\text { CEBPA,CEBPB,C } \\
\text { EBPD,HLA- } \\
\text { DQA1,HMOX1,S } \\
\text { OX8,PLEKHB1,H } \\
\text { MG20B,S100A1,F } \\
\text { ZD9,HIST2H2AA } \\
\text { 3,ADRB2,PRMT2 } \\
\text { „PLEK,PLOD1,A } \\
\text { LOX5AP,VEGFB, } \\
\text { S100A16,APOC2, } \\
\text { BCAS1,APOE,CR } \\
\text { YAB,SLC11A1,C } \\
\text { SF1R,PYCARD, } \\
\text { MAPK4,CYBA,M }\end{array}$ \\
\hline
\end{tabular}


medRxiv preprint doi: https://doi.org/10.1101/2020.12.21.20248688; this version posted December 24, 2020. The copyright holder for this preprint (which was not certified by peer review) is the author/funder, who has granted medRxiv a license to display the preprint in perpetuity. All rights reserved. No reuse allowed without permission.

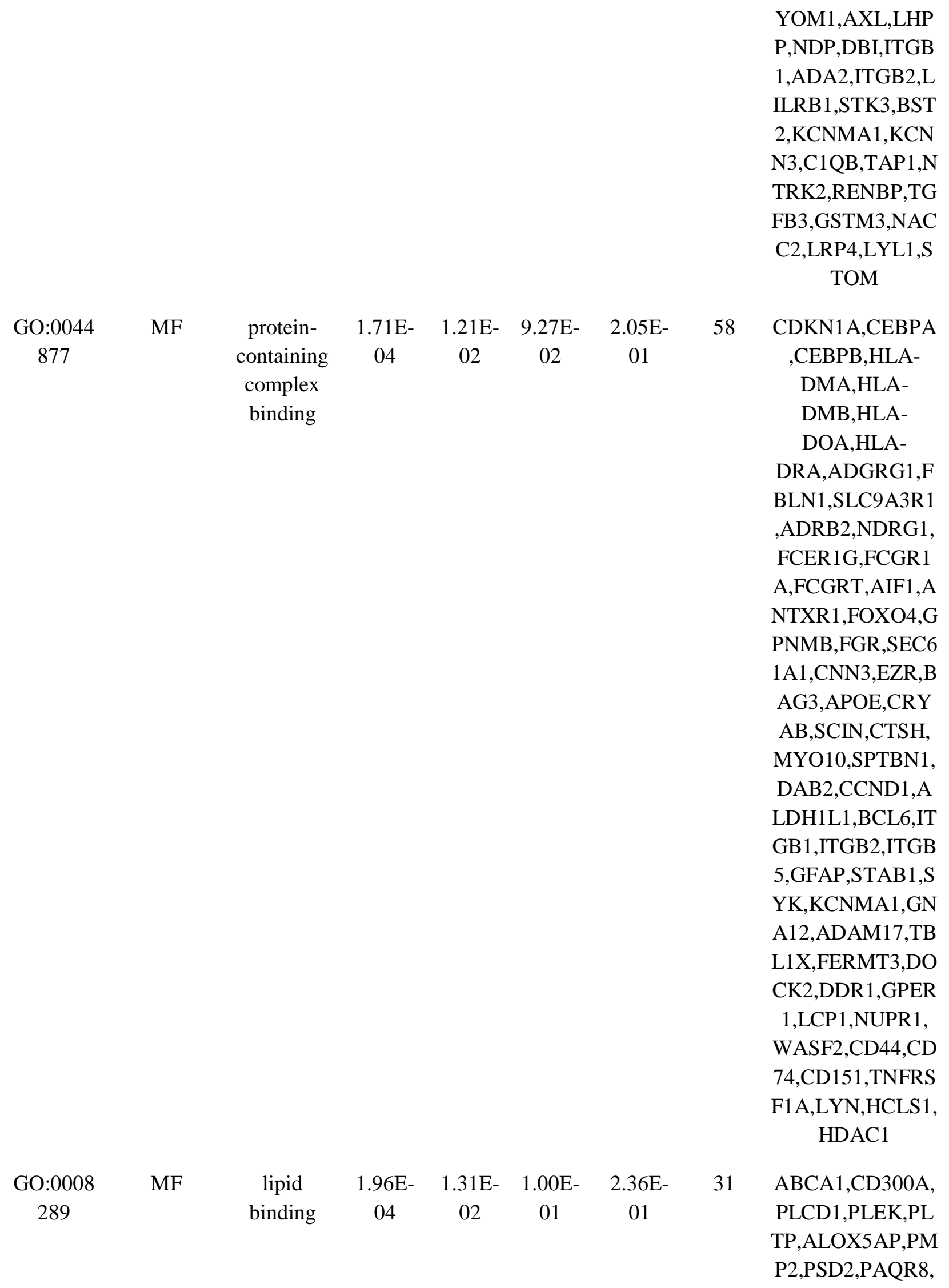


medRxiv preprint doi: https://doi.org/10.1101/2020.12.21.20248688; this version posted December 24, 2020. The copyright holder for this preprint (which was not certified by peer review) is the author/funder, who has granted medRxiv a license to display the preprint in perpetuity. All rights reserved. No reuse allowed without permission.

\begin{tabular}{|c|c|c|c|c|c|c|c|c|}
\hline $\begin{array}{c}\text { GO:0004 } \\
888\end{array}$ & $\mathrm{MF}$ & $\begin{array}{c}\text { transmem } \\
\text { branesigna } \\
\text { ling } \\
\text { receptor } \\
\text { activity }\end{array}$ & $\begin{array}{c}8.58 \mathrm{E}- \\
04\end{array}$ & $\begin{array}{c}3.82 \mathrm{E}- \\
02\end{array}$ & $\begin{array}{c}2.93 \mathrm{E}- \\
01\end{array}$ & $\begin{array}{c}1.00 \mathrm{E}+0 \\
0\end{array}$ & 47 & $\begin{array}{c}\text { ABCA1,HLA- } \\
\text { DOA,HLA- } \\
\text { DPA1,HLA- } \\
\text { DQA1,HLA- } \\
\text { DRA,GPR37L1,A } \\
\text { DGRG1,ADGRV1 } \\
\text {,RYR3,FZD9,AD } \\
\text { RB2,FCER1G,FC } \\
\text { GR1A,FCGRT,AP } \\
\text { LNR,ANTXR1,F } \\
\text { GFR3,MERTK,IL } \\
\text { 17RB,PLXNB1,IF } \\
\text { NGR1,CSF1R,CS } \\
\text { F2RA,CSF3R,CT } \\
\text { SH,GPRC5B,IL13 } \\
\text { RA1,AXL,FGFRL } \\
\text { 1,LHPP,ITGB2,LI } \\
\text { LRB1,PTH1R,PT } \\
\text { PN6,DDR1,LILR } \\
\text { B3,LPAR5,GPER } \\
\text { 1,NTRK2,GRIN2 } \\
\text { C,LPAR1,CD14,E } \\
\text { B13,TLR5,CD44, } \\
\text { CD74,TNFRSF1A }\end{array}$ \\
\hline
\end{tabular}

Table 6 Topology table for up and down regulated genes

\begin{tabular}{ccccccc}
\hline Regulation & Node & Degree & Betweenness & Stress & Closeness & $\begin{array}{c}\text { Clustering } \\
\text { Coefficient }\end{array}$ \\
\hline Up & YWHAZ & 520 & 0.303479 & 34572544 & 0.418391 & 0.003112 \\
Up & GABARAPL1 & 519 & 0.223347 & 21852052 & 0.377829 & $9.97 \mathrm{E}-04$ \\
$\mathrm{Up}$ & SSX2IP & 207 & 0.093692 & 9473730 & 0.347627 & 0.001595
\end{tabular}


medRxiv preprint doi: https://doi.org/10.1101/2020.12.21.20248688; this version posted December 24, 2020. The copyright holder for this preprint (which was not certified by peer review) is the author/funder, who has granted medRxiv a license to display the preprint in perpetuity.

All rights reserved. No reuse allowed without permission.

\begin{tabular}{|c|c|c|c|c|c|c|}
\hline Up & YWHAH & 185 & 0.049951 & 7280666 & 0.345909 & 0.007638 \\
\hline Up & UBE2N & 176 & 0.078233 & 11122364 & 0.323757 & 0.001623 \\
\hline Up & CALM3 & 148 & 0.06597 & 7100308 & 0.347196 & 0.002758 \\
\hline Up & PNMA1 & 130 & 0.057117 & 5261682 & 0.340441 & 0.002504 \\
\hline Up & MAGED1 & 127 & 0.053559 & 6137200 & 0.321214 & 0.003375 \\
\hline Up & TUBB3 & 126 & 0.047797 & 8153956 & 0.354741 & 0.01054 \\
\hline Up & USP11 & 90 & 0.03642 & 4902718 & 0.311991 & 4.99E-04 \\
\hline Up & STX1A & 88 & 0.039459 & 2972380 & 0.287452 & 0.003135 \\
\hline Up & LDOC1 & 83 & 0.025842 & 5269048 & 0.306836 & $5.88 \mathrm{E}-04$ \\
\hline Up & FHL2 & 83 & 0.035779 & 5436576 & 0.302552 & 0 \\
\hline Up & APP & 83 & 0.042781 & 4166770 & 0.341048 & 0.007346 \\
\hline $\mathrm{Up}$ & EPB41L3 & 82 & 0.033089 & 2837640 & 0.340346 & 0.012045 \\
\hline $\mathrm{Up}$ & ATP6V1A & 72 & 0.025583 & 3674416 & 0.299835 & 0.005869 \\
\hline Up & DCLK1 & 69 & 0.019955 & 6604820 & 0.28752 & 0 \\
\hline Up & SNCA & 67 & 0.022716 & 2822196 & 0.316329 & 0.003166 \\
\hline $\mathrm{Up}$ & RAN & 59 & 0.016754 & 2753448 & 0.30389 & 0 \\
\hline Up & MAPK9 & 59 & 0.022167 & 4416440 & 0.303485 & 0.001753 \\
\hline Up & $\mathrm{BEX} 2$ & 58 & 0.023183 & 1906394 & 0.302225 & 0.00363 \\
\hline Up & TRIM37 & 56 & 0.01734 & 2325384 & 0.295575 & 0.01039 \\
\hline Up & PRNP & 54 & 0.022786 & 1450206 & 0.298924 & $6.99 \mathrm{E}-04$ \\
\hline Up & CDK5 & 53 & 0.016119 & 5560370 & 0.288523 & 0 \\
\hline Up & MAP2K1 & 51 & 0.018383 & 1488872 & 0.316604 & 0.009412 \\
\hline Up & PAK1 & 50 & 0.017919 & 1287682 & 0.323699 & 0.007347 \\
\hline Up & PRKAR1B & 49 & 0.014937 & 2523534 & 0.30519 & 0 \\
\hline Up & SLC38A1 & 48 & 0.018191 & 2825680 & 0.277905 & 8.87E-04 \\
\hline Up & SH3GL2 & 48 & 0.017084 & 3543090 & 0.283976 & 0.003546 \\
\hline Up & NSF & 47 & 0.017194 & 1375530 & 0.30125 & $9.25 \mathrm{E}-04$ \\
\hline Up & DDX24 & 44 & 0.014803 & 1817222 & 0.308213 & 0.002114 \\
\hline Up & RBFOX2 & 42 & 0.009651 & 1205330 & 0.298826 & 0.026713 \\
\hline Up & CCNA1 & 42 & 0.012785 & 1332634 & 0.281603 & 0.001161 \\
\hline Up & ATP1A1 & 41 & 0.011681 & 1956120 & 0.308632 & 0.004878 \\
\hline Up & TUBB2A & 39 & 0.014824 & 1564672 & 0.333394 & 0.014845 \\
\hline $\mathrm{Up}$ & RTN4 & 36 & 0.009227 & 2635674 & 0.276428 & 0.011111 \\
\hline Up & PFN2 & 36 & 0.017354 & 1554706 & 0.31151 & 0.006349 \\
\hline Up & GPRASP2 & 35 & 0.009795 & 1454950 & 0.28549 & 0 \\
\hline $\mathrm{Up}$ & PNMA2 & 35 & 0.009209 & 1124876 & 0.325261 & 0.028571 \\
\hline Up & RNF41 & 35 & 0.013898 & 2481286 & 0.253747 & 0 \\
\hline Up & ATP2A2 & 34 & 0.007725 & 1606958 & 0.303814 & 0 \\
\hline Up & TSPYL2 & 33 & 0.011521 & 1837306 & 0.260074 & 0 \\
\hline $\mathrm{Up}$ & TPM1 & 33 & 0.007946 & 820602 & 0.316742 & 0.015152 \\
\hline Up & CHGB & 32 & 0.008919 & 1181836 & 0.285939 & 0.004032 \\
\hline Up & MAP1B & 31 & 0.00804 & 1759306 & 0.28944 & 0 \\
\hline Up & DYNC1I1 & 31 & 0.008074 & 1435618 & 0.286614 & 0 \\
\hline
\end{tabular}


medRxiv preprint doi: https://doi.org/10.1101/2020.12.21.20248688; this version posted December 24, 2020. The copyright holder for this preprint (which was not certified by peer review) is the author/funder, who has granted medRxiv a license to display the preprint in perpetuity.

All rights reserved. No reuse allowed without permission.

\begin{tabular}{|c|c|c|c|c|c|c|}
\hline Up & ALDOA & 31 & 0.008241 & 1028948 & 0.325174 & 0.023656 \\
\hline Up & STAMBPL1 & 30 & 0.010007 & 826570 & 0.295839 & 0 \\
\hline Up & HPRT1 & 29 & 0.010272 & 1194692 & 0.318823 & 0.03202 \\
\hline Up & $\mathrm{ENO} 2$ & 29 & 0.010363 & 749602 & 0.302426 & 0.019704 \\
\hline Up & CIRBP & 29 & 0.013379 & 945212 & 0.31846 & 0.007389 \\
\hline Up & SMYD2 & 28 & 0.008525 & 891396 & 0.288248 & 0.002646 \\
\hline Up & TUBA1B & 28 & 0.006355 & 679812 & 0.323613 & 0.044974 \\
\hline Up & G3BP2 & 28 & 0.010082 & 854254 & 0.292416 & 0.005291 \\
\hline Up & UCHL1 & 28 & 0.005862 & 928260 & 0.305088 & 0.002646 \\
\hline Up & $\mathrm{KLC1}$ & 27 & 0.005385 & 375808 & 0.311991 & 0.054131 \\
\hline Up & IARS & 27 & 0.004513 & 420104 & 0.318488 & 0.019943 \\
\hline Up & DNM1 & 27 & 0.010115 & 1015404 & 0.29917 & 0.022792 \\
\hline Up & VPS35 & 26 & 0.006859 & 1341624 & 0.290456 & 0 \\
\hline Up & PGRMC1 & 26 & 0.006398 & 1609490 & 0.27219 & 0 \\
\hline Up & NME1 & 26 & 0.009137 & 742508 & 0.305574 & 0.003077 \\
\hline Up & MAL2 & 25 & 0.007673 & 873010 & 0.261588 & 0 \\
\hline Up & RBFOX1 & 25 & 0.00641 & 480594 & 0.285939 & 0.04 \\
\hline Up & GNB5 & 24 & 0.007755 & 1759018 & 0.258394 & 0 \\
\hline Up & NEFL & 24 & 0.006714 & 514106 & 0.300033 & 0.007246 \\
\hline Up & BEX1 & 23 & 0.003327 & 509968 & 0.252989 & 0 \\
\hline Up & TOMM20 & 22 & 0.005795 & 846012 & 0.288089 & 0 \\
\hline Up & GAS7 & 22 & 0.004679 & 548152 & 0.274655 & 0.030303 \\
\hline Up & CAMK2B & 22 & 0.006891 & 530376 & 0.276302 & 0.012987 \\
\hline Up & PACSIN1 & 21 & 0.007754 & 550504 & 0.273581 & 0.004762 \\
\hline Up & NCKAP1 & 21 & 0.005268 & 462816 & 0.308658 & 0.104762 \\
\hline Up & SLC25A12 & 21 & 0.006082 & 850432 & 0.288591 & 0 \\
\hline Up & SNRPN & 21 & 0.006083 & 1067330 & 0.267962 & 0 \\
\hline Up & OAT & 21 & 0.005568 & 866682 & 0.286795 & 0.004762 \\
\hline Up & EGR1 & 21 & 0.006159 & 816024 & 0.259555 & 0 \\
\hline Up & WASF1 & 20 & 0.003276 & 302586 & 0.283865 & 0.042105 \\
\hline Up & ATP6V1B2 & 20 & 0.002662 & 462898 & 0.283954 & 0.057895 \\
\hline Up & RUNDC3A & 19 & 0.004563 & 1118982 & 0.242748 & 0 \\
\hline Up & BASP1 & 19 & 0.001808 & 349670 & 0.279634 & 0 \\
\hline Up & RTN3 & 19 & 0.005329 & 640152 & 0.266024 & 0.040936 \\
\hline Up & CYFIP2 & 18 & 0.004289 & 338978 & 0.314933 & 0.117647 \\
\hline Up & NEFM & 18 & 0.003956 & 565288 & 0.290155 & 0.006536 \\
\hline Up & MAP4 & 17 & 0.002527 & 559320 & 0.289786 & 0.022059 \\
\hline Up & NAPB & 16 & 0.003369 & 301488 & 0.26026 & 0.066667 \\
\hline Up & PLEKHB2 & 16 & 0.003117 & 385238 & 0.248787 & 0 \\
\hline Up & NELL1 & 16 & 0.004613 & 609270 & 0.272823 & 0 \\
\hline Up & STRBP & 15 & 0.002031 & 377222 & 0.2567 & 0 \\
\hline $\mathrm{Up}$ & ARHGAP32 & 15 & 0.003077 & 453612 & 0.27727 & 0 \\
\hline Up & TSPYL4 & 14 & 0.003989 & 305642 & 0.268913 & 0 \\
\hline
\end{tabular}


medRxiv preprint doi: https://doi.org/10.1101/2020.12.21.20248688; this version posted December 24, 2020. The copyright holder for this preprint (which was not certified by peer review) is the author/funder, who has granted medRxiv a license to display the preprint in perpetuity. All rights reserved. No reuse allowed without permission.

\begin{tabular}{|c|c|c|c|c|c|c|}
\hline Up & STXBP1 & 14 & 0.004421 & 323342 & 0.284197 & 0 \\
\hline Up & MAP2K4 & 14 & 0.003232 & 317308 & 0.273664 & 0.032967 \\
\hline Up & PI4KA & 14 & 0.002273 & 454832 & 0.27354 & 0 \\
\hline $\mathrm{Up}$ & FKBP1A & 14 & 0.003669 & 523150 & 0.249264 & 0 \\
\hline Up & ACOT7 & 13 & 0.002579 & 311680 & 0.275967 & 0.012821 \\
\hline Up & STMN2 & 13 & 0.003402 & 382796 & 0.26939 & 0 \\
\hline Up & PRKCB & 13 & 0.003077 & 583720 & 0.270471 & 0 \\
\hline Up & CDK14 & 13 & 0.001632 & 182338 & 0.282018 & 0.038462 \\
\hline Up & ETS2 & 13 & 0.005562 & 880622 & 0.219185 & 0 \\
\hline Up & ATP1B1 & 13 & 0.003131 & 202320 & 0.269131 & 0.051282 \\
\hline $\mathrm{Up}$ & NGEF & 12 & 0.003038 & 516072 & 0.244772 & 0 \\
\hline Up & РРP3СВ & 12 & 0.004548 & 671290 & 0.264132 & 0 \\
\hline Up & PPP1R2 & 12 & 0.002333 & 310394 & 0.270592 & 0 \\
\hline Up & PGAM1 & 12 & 0.002562 & 240280 & 0.316687 & 0.090909 \\
\hline Up & STMN1 & 12 & 0.001708 & 359304 & 0.266862 & 0 \\
\hline Up & ATP6V1E1 & 12 & 0.001868 & 216802 & 0.283423 & 0.060606 \\
\hline Up & ATP6V0C & 12 & 0.00329 & 332358 & 0.257663 & 0 \\
\hline Up & $\mathrm{ACP} 1$ & 12 & 0.001327 & 278778 & 0.251503 & 0 \\
\hline Up & NAP1L5 & 11 & 0.001162 & 254090 & 0.261682 & 0 \\
\hline Up & PJA1 & 11 & 0.002953 & 176012 & 0.272986 & 0 \\
\hline Up & EPDR1 & 11 & 0.003347 & 197776 & 0.288317 & 0.018182 \\
\hline Up & SYT1 & 11 & 0.002389 & 382104 & 0.261532 & 0 \\
\hline Up & SYN1 & 11 & 0.002727 & 257626 & 0.281908 & 0.036364 \\
\hline Up & VAMP2 & 11 & 0.004158 & 413696 & 0.253253 & 0.036364 \\
\hline Up & PRKCE & 11 & 0.002925 & 206314 & 0.306759 & 0.072727 \\
\hline Up & KIF3C & 11 & 0.002403 & 368746 & 0.26394 & 0 \\
\hline Up & CKMT1A & 10 & 0.001142 & 172006 & 0.313712 & 0.022222 \\
\hline $\mathrm{Up}$ & CADPS2 & 10 & 0.002927 & 328096 & 0.24794 & 0.044444 \\
\hline Up & AKAP6 & 10 & $9.40 \mathrm{E}-04$ & 284338 & 0.251712 & 0 \\
\hline Up & KALRN & 10 & 0.001181 & 171202 & 0.265132 & 0 \\
\hline Up & PTPRN & 10 & 0.002201 & 282330 & 0.254368 & 0 \\
\hline $\mathrm{Up}$ & PPP3R1 & 10 & 0.00278 & 245790 & 0.26989 & 0.022222 \\
\hline Up & MAP2 & 10 & $7.24 \mathrm{E}-04$ & 164684 & 0.264036 & 0 \\
\hline Up & ICA1 & 10 & 0.003465 & 416502 & 0.244246 & 0 \\
\hline Up & GAD1 & 10 & 0.00268 & 295646 & 0.276638 & 0 \\
\hline Up & СКМТ1B & 10 & 0.001142 & 172006 & 0.313712 & 0.022222 \\
\hline Up & ASNS & 10 & 0.002036 & 342344 & 0.277926 & 0 \\
\hline Up & AMPH & 10 & 0.002749 & 268180 & 0.250516 & 0.022222 \\
\hline Up & MOAP1 & 9 & 0.001934 & 233876 & 0.233618 & 0 \\
\hline Up & AGK & 9 & 0.001298 & 179476 & 0.269251 & 0 \\
\hline Up & BCL11A & 9 & 0.003862 & 355102 & 0.221371 & 0 \\
\hline $\mathrm{Up}$ & SLIT2 & 9 & 0.002987 & 411796 & 0.249606 & 0 \\
\hline Up & OPA1 & 9 & 0.001878 & 204586 & 0.269291 & 0 \\
\hline
\end{tabular}


medRxiv preprint doi: https://doi.org/10.1101/2020.12.21.20248688; this version posted December 24, 2020. The copyright holder for this preprint (which was not certified by peer review) is the author/funder, who has granted medRxiv a license to display the preprint in perpetuity. All rights reserved. No reuse allowed without permission.

\begin{tabular}{|c|c|c|c|c|c|c|}
\hline Up & MDH1 & 9 & 0.004987 & 785188 & 0.28752 & 0 \\
\hline Up & ENSA & 9 & 0.002265 & 245204 & 0.253165 & 0.055556 \\
\hline Up & AP1S1 & 9 & 0.002823 & 295576 & 0.243511 & 0 \\
\hline Up & CCKBR & 9 & 0.003224 & 588778 & 0.253765 & 0 \\
\hline Up & SCAMP5 & 8 & $2.25 \mathrm{E}-04$ & 33196 & 0.202887 & 0 \\
\hline Up & MRAP2 & 8 & 0.003843 & 293958 & 0.192257 & 0 \\
\hline Up & CAMK1D & 8 & 0.001706 & 334732 & 0.25081 & 0 \\
\hline Up & DCAF6 & 8 & $6.09 \mathrm{E}-04$ & 118754 & 0.265345 & 0 \\
\hline Up & ATP6V1H & 8 & 0.002212 & 204098 & 0.252235 & 0 \\
\hline Up & AK5 & 8 & 0.003542 & 207288 & 0.295551 & 0 \\
\hline $\mathrm{Up}$ & CAP2 & 8 & 0.001797 & 406950 & 0.248821 & 0 \\
\hline Up & LDB2 & 8 & 0.003865 & 399054 & 0.239285 & 0 \\
\hline Up & SCG2 & 8 & $9.38 \mathrm{E}-04$ & 152816 & 0.257991 & 0 \\
\hline Up & SNAP25 & 8 & 0.003163 & 188104 & 0.267293 & 0.071429 \\
\hline Up & DOCK3 & 8 & $6.55 \mathrm{E}-04$ & 167712 & 0.250499 & 0 \\
\hline Up & CAMK2G & 8 & 0.001384 & 261198 & 0.266608 & 0 \\
\hline Up & MAP7D2 & 7 & $2.40 \mathrm{E}-04$ & 57370 & 0.269052 & 0 \\
\hline Up & NCOA7 & 7 & 0.001189 & 101368 & 0.280388 & 0.095238 \\
\hline Up & NECAB1 & 7 & 0.001294 & 131820 & 0.256718 & 0 \\
\hline Up & LIN7B & 7 & 0.003294 & 389118 & 0.182804 & 0 \\
\hline Up & DIRAS2 & 7 & $6.20 \mathrm{E}-04$ & 135562 & 0.290017 & 0.047619 \\
\hline Up & FBXL2 & 7 & 0.001674 & 247096 & 0.249708 & 0 \\
\hline Up & NRXN1 & 7 & 0.002222 & 173864 & 0.209015 & 0 \\
\hline Up & INA & 7 & $3.53 \mathrm{E}-04$ & 53202 & 0.278245 & 0 \\
\hline Up & TARBP1 & 7 & 0.001206 & 217698 & 0.240629 & 0 \\
\hline Up & TAC1 & 7 & 0.002023 & 242786 & 0.247148 & 0 \\
\hline Up & FGF12 & 7 & 0.001831 & 218342 & 0.230686 & 0 \\
\hline Up & EPB41L1 & 7 & $5.22 \mathrm{E}-04$ & 71492 & 0.277863 & 0.047619 \\
\hline Up & ATP2B1 & 7 & 0.001202 & 106770 & 0.259611 & 0.047619 \\
\hline Up & LINGO1 & 6 & 0.001131 & 130154 & 0.215704 & 0 \\
\hline Up & ACTR10 & 6 & $5.83 \mathrm{E}-04$ & 57200 & 0.228399 & 0 \\
\hline Up & TMEM14A & 6 & $6.03 \mathrm{E}-04$ & 57288 & 0.243429 & 0 \\
\hline Up & PNKD & 6 & 0.001189 & 151034 & 0.220834 & 0 \\
\hline Up & MYRIP & 6 & 0.001137 & 192738 & 0.245614 & 0 \\
\hline Up & TBR1 & 6 & 0.00117 & 152722 & 0.266667 & 0 \\
\hline Up & ELMO1 & 6 & 0.001682 & 278946 & 0.221425 & 0 \\
\hline Up & PRSS3 & 6 & 0.001172 & 135896 & 0.266921 & 0 \\
\hline Up & PRKCG & 6 & $8.09 \mathrm{E}-04$ & 123106 & 0.251416 & 0 \\
\hline Up & NPY & 6 & 0.001415 & 255346 & 0.2221 & 0 \\
\hline Up & ITPR1 & 6 & 0.001152 & 122148 & 0.24492 & 0 \\
\hline Up & CHN1 & 6 & 0.001678 & 267066 & 0.21527 & 0 \\
\hline Up & ZCCHC12 & 5 & $9.05 \mathrm{E}-04$ & 78548 & 0.258065 & 0 \\
\hline Up & FBXO34 & 5 & $9.49 \mathrm{E}-04$ & 134314 & 0.256555 & 0 \\
\hline
\end{tabular}


medRxiv preprint doi: https://doi.org/10.1101/2020.12.21.20248688; this version posted December 24, 2020. The copyright holder for this preprint (which was not certified by peer review) is the author/funder, who has granted medRxiv a license to display the preprint in perpetuity. All rights reserved. No reuse allowed without permission.

\begin{tabular}{|c|c|c|c|c|c|c|}
\hline Up & OCIAD1 & 5 & 0.001651 & 186292 & 0.208787 & 0 \\
\hline Up & RAB6B & 5 & 0.001151 & 138186 & 0.243788 & 0 \\
\hline Up & PNMA3 & 5 & 7.09E-04 & 78626 & 0.252866 & 0 \\
\hline $\mathrm{Up}$ & SULT4A1 & 5 & $5.98 \mathrm{E}-04$ & 89296 & 0.239616 & 0 \\
\hline Up & CELF2 & 5 & $5.80 \mathrm{E}-04$ & 105312 & 0.260614 & 0 \\
\hline Up & PREPL & 5 & 0.001183 & 199254 & 0.220366 & 0 \\
\hline Up & NREP & 5 & 7.49E-04 & 74980 & 0.243088 & 0 \\
\hline Up & CNTNAP1 & 5 & $5.93 \mathrm{E}-05$ & 15268 & 0.247081 & 0 \\
\hline $\mathrm{Up}$ & VSNL1 & 5 & 0.001258 & 130854 & 0.239285 & 0 \\
\hline Up & TSPYL1 & 5 & $8.01 \mathrm{E}-04$ & 97912 & 0.252673 & 0 \\
\hline $\mathrm{Up}$ & STAT4 & 5 & 0.002197 & 229156 & 0.207561 & 0 \\
\hline Up & SCG5 & 5 & $6.39 \mathrm{E}-04$ & 86632 & 0.225066 & 0 \\
\hline Up & RGS7 & 5 & $3.10 \mathrm{E}-04$ & 46406 & 0.259518 & 0 \\
\hline Up & PTPRN2 & 5 & $9.33 \mathrm{E}-04$ & 129298 & 0.253782 & 0 \\
\hline Up & PCP4 & 5 & $6.13 \mathrm{E}-04$ & 121714 & 0.242424 & 0 \\
\hline Up & OPCML & 5 & 8.01E-04 & 88028 & 0.256971 & 0 \\
\hline Up & NELL2 & 5 & $1.19 \mathrm{E}-04$ & 18698 & 0.241299 & 0 \\
\hline Up & GNAO1 & 5 & 0.001142 & 95648 & 0.271439 & 0 \\
\hline Up & GLS & 5 & $1.54 \mathrm{E}-04$ & 23754 & 0.242796 & 0 \\
\hline Up & GAD2 & 5 & $6.64 \mathrm{E}-04$ & 47922 & 0.236456 & 0 \\
\hline Up & GABRD & 5 & 0.001134 & 155676 & 0.223203 & 0 \\
\hline Up & CHRM1 & 5 & $8.90 \mathrm{E}-04$ & 92394 & 0.253942 & 0 \\
\hline Up & ADARB1 & 5 & 0.001109 & 134272 & 0.252322 & 0 \\
\hline Up & FBXL16 & 4 & $5.65 \mathrm{E}-04$ & 50880 & 0.239175 & 0 \\
\hline Up & MFSD6 & 4 & $2.17 \mathrm{E}-06$ & 322 & 0.257499 & 0.5 \\
\hline Up & NT5DC3 & 4 & $8.11 \mathrm{E}-04$ & 83504 & 0.214711 & 0 \\
\hline Up & CNTNAP2 & 4 & 0.001108 & 322366 & 0.228042 & 0 \\
\hline Up & NECAP1 & 4 & $8.51 \mathrm{E}-04$ & 62830 & 0.243348 & 0 \\
\hline Up & FAM3C & 4 & $5.09 \mathrm{E}-04$ & 18328 & 0.226481 & 0 \\
\hline Up & GABBR2 & 4 & $3.10 \mathrm{E}-04$ & 13894 & 0.23431 & 0.166667 \\
\hline Up & HERC1 & 4 & $3.93 \mathrm{E}-05$ & 13426 & 0.263596 & 0 \\
\hline Up & SNCB & 4 & $5.75 \mathrm{E}-04$ & 51258 & 0.223587 & 0 \\
\hline Up & PTPRR & 4 & $6.02 \mathrm{E}-04$ & 39492 & 0.206513 & 0 \\
\hline Up & GOT1 & 4 & $7.09 \mathrm{E}-04$ & 79346 & 0.251069 & 0 \\
\hline Up & АTP2B2 & 4 & 0.001112 & 113994 & 0.246897 & 0 \\
\hline Up & ARF3 & 4 & $2.47 \mathrm{E}-04$ & 49364 & 0.24464 & 0 \\
\hline Up & PGM2L1 & 3 & $5.61 \mathrm{E}-04$ & 29146 & 0.199813 & 0 \\
\hline Up & CYGB & 3 & 0.001099 & 180094 & 0.176511 & 0 \\
\hline Up & NCALD & 3 & $5.84 \mathrm{E}-04$ & 74964 & 0.194496 & 0 \\
\hline Up & MAGEE1 & 3 & $5.74 \mathrm{E}-04$ & 46822 & 0.237567 & 0 \\
\hline Up & TMX4 & 3 & $5.59 \mathrm{E}-04$ & 69560 & 0.226961 & 0 \\
\hline Up & SYBU & 3 & $6.59 \mathrm{E}-04$ & 71018 & 0.237335 & 0 \\
\hline Up & CALY & 3 & 0.001099 & 72922 & 0.202639 & 0 \\
\hline
\end{tabular}


medRxiv preprint doi: https://doi.org/10.1101/2020.12.21.20248688; this version posted December 24, 2020. The copyright holder for this preprint (which was not certified by peer review) is the author/funder, who has granted medRxiv a license to display the preprint in perpetuity. All rights reserved. No reuse allowed without permission.

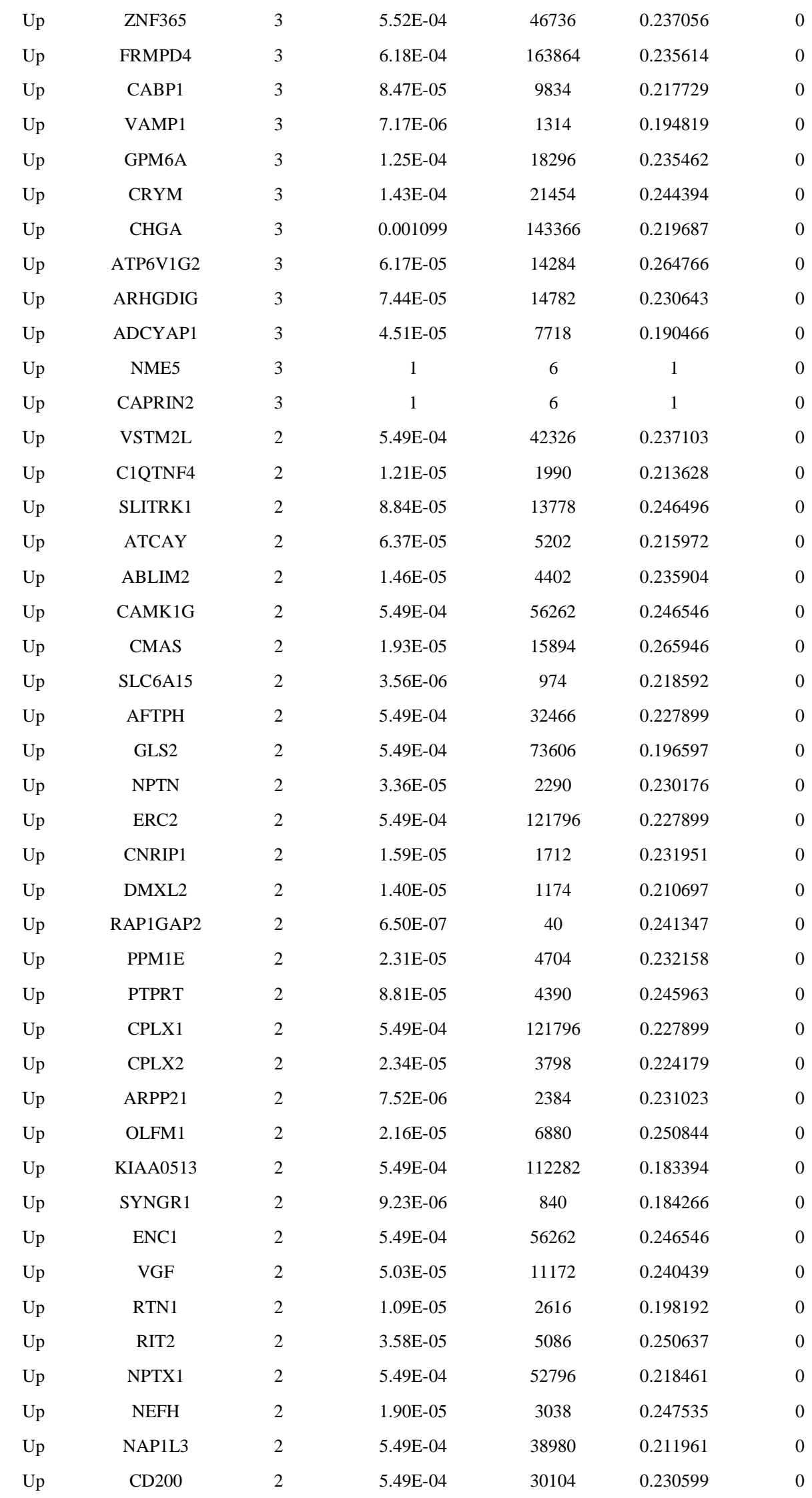


medRxiv preprint doi: https://doi.org/10.1101/2020.12.21.20248688; this version posted December 24, 2020. The copyright holder for this preprint (which was not certified by peer review) is the author/funder, who has granted medRxiv a license to display the preprint in perpetuity. All rights reserved. No reuse allowed without permission.

\begin{tabular}{|c|c|c|c|c|c|}
\hline Up & GNG3 & 2 & $2.08 \mathrm{E}-05$ & 1398 & 0.214181 \\
\hline Up & EPHA4 & 2 & $9.67 \mathrm{E}-06$ & 2466 & 0.25596 \\
\hline Up & ELAVL4 & 2 & $5.49 \mathrm{E}-04$ & 43134 & 0.220259 \\
\hline Up & ATP2B3 & 2 & $5.49 \mathrm{E}-04$ & 56550 & 0.241347 \\
\hline Up & BEX5 & 2 & 1 & 2 & 1 \\
\hline Up & SV2B & 2 & 1 & 2 & 1 \\
\hline Up & KCNIP4 & 2 & 1 & 2 & 1 \\
\hline Up & CACNB2 & 2 & 1 & 2 & 1 \\
\hline Up & ARHGAP44 & 2 & 1 & 2 & 1 \\
\hline Up & KCTD8 & 1 & 0 & 0 & 0.246512 \\
\hline Up & NRSN1 & 1 & 0 & 0 & 0.180807 \\
\hline Up & CBLN4 & 1 & 0 & 0 & 0.218055 \\
\hline Up & SLC32A1 & 1 & 0 & 0 & 0.25889 \\
\hline Up & KRT222 & 1 & 0 & 0 & 0.205105 \\
\hline Up & HENMT1 & 1 & 0 & 0 & 0.21555 \\
\hline Up & CEP41 & 1 & 0 & 0 & 0.205371 \\
\hline Up & C16orf45 & 1 & 0 & 0 & 0.232217 \\
\hline Up & PNMA6A & 1 & 0 & 0 & 0.253995 \\
\hline Up & CORO6 & 1 & 0 & 0 & 0.182557 \\
\hline Up & SPHKAP & 1 & 0 & 0 & 0.188533 \\
\hline Up & RASL11B & 1 & 0 & 0 & 0.220232 \\
\hline Up & REEP1 & 1 & 0 & 0 & 0.257026 \\
\hline Up & DPP10 & 1 & 0 & 0 & 0.217808 \\
\hline Up & PITHD1 & 1 & 0 & 0 & 0.208155 \\
\hline Up & PDXP & 1 & 0 & 0 & 0.237629 \\
\hline Up & GNG2 & 1 & 0 & 0 & 0.207561 \\
\hline Up & PCSK1N & 1 & 0 & 0 & 0.172864 \\
\hline Up & PARM1 & 1 & 0 & 0 & 0.198961 \\
\hline Up & FLRT3 & 1 & 0 & 0 & 0.188533 \\
\hline Up & CAMTA1 & 1 & 0 & 0 & 0.215691 \\
\hline Up & RCAN2 & 1 & 0 & 0 & 0.174497 \\
\hline Up & CLSTN3 & 1 & 0 & 0 & 0.202797 \\
\hline Up & ADAM23 & 1 & 0 & 0 & 0.295 \\
\hline Up & AP3B2 & 1 & 0 & 0 & 0.228945 \\
\hline Up & TUSC3 & 1 & 0 & 0 & 0.196874 \\
\hline Up & FZD3 & 1 & 0 & 0 & 0.220901 \\
\hline Up & SYP & 1 & 0 & 0 & 0.230147 \\
\hline Up & SYN2 & 1 & 0 & 0 & 0.22787 \\
\hline Up & RGS4 & 1 & 0 & 0 & 0.248821 \\
\hline Up & PDE1A & 1 & 0 & 0 & 0.295 \\
\hline Up & CNTN3 & 1 & 0 & 0 & 0.193792 \\
\hline Up & MAР3К9 & 1 & 0 & 0 & 0.246512 \\
\hline Up & $\mathrm{KCNJ} 4$ & 1 & 0 & 0 & 0.154558 \\
\hline
\end{tabular}


medRxiv preprint doi: https://doi.org/10.1101/2020.12.21.20248688; this version posted December 24, 2020. The copyright holder for this preprint (which was not certified by peer review) is the author/funder, who has granted medRxiv a license to display the preprint in perpetuity. All rights reserved. No reuse allowed without permission.

\begin{tabular}{|c|c|c|c|c|c|c|}
\hline Up & HPCA & 1 & 0 & 0 & 0.189662 & 0 \\
\hline Up & EXTL2 & 1 & 0 & 0 & 0.197848 & 0 \\
\hline Up & DPP6 & 1 & 0 & 0 & 0.217015 & 0 \\
\hline Up & DLX1 & 1 & 0 & 0 & 0.161864 & 0 \\
\hline Up & COL5A2 & 1 & 0 & 0 & 0.20391 & 0 \\
\hline Up & CCK & 1 & 0 & 0 & 0.198961 & 0 \\
\hline Down & HSPB 1 & 297 & 0.252553 & 21161044 & 0.352134 & $7.28 \mathrm{E}-04$ \\
\hline Down & HDAC1 & 234 & 0.147861 & 14084732 & 0.317228 & $3.67 \mathrm{E}-04$ \\
\hline Down & CDKN1A & 159 & 0.117762 & 9153794 & 0.318419 & 0.001274 \\
\hline Down & TNFRSF1A & 116 & 0.068603 & 4417914 & 0.302187 & $9.00 \mathrm{E}-04$ \\
\hline Down & FKBP5 & 94 & 0.059602 & 5497602 & 0.298757 & $4.58 \mathrm{E}-04$ \\
\hline Down & BCL6 & 85 & 0.04794 & 5770508 & 0.271764 & 0 \\
\hline Down & STOM & 77 & 0.042309 & 4531172 & 0.273566 & 0 \\
\hline Down & ТР53BP2 & 74 & 0.038647 & 5370676 & 0.277017 & 0 \\
\hline Down & STK3 & 71 & 0.039036 & 4265582 & 0.278838 & 0.002817 \\
\hline Down & BAG3 & 68 & 0.049404 & 2666838 & 0.307112 & 0.007463 \\
\hline Down & TEAD2 & 66 & 0.03415 & 3396934 & 0.253761 & 0 \\
\hline Down & SPTBN1 & 63 & 0.039882 & 4787616 & 0.315947 & 0.004608 \\
\hline Down & NEK6 & 61 & 0.031053 & 3457996 & 0.273077 & $5.46 \mathrm{E}-04$ \\
\hline Down & NDRG1 & 61 & 0.027742 & 3297714 & 0.274107 & 0 \\
\hline Down & GFAP & 60 & 0.032796 & 5105400 & 0.282581 & 0 \\
\hline Down & HAP1 & 58 & 0.032839 & 2490564 & 0.246408 & 0 \\
\hline Down & HCK & 53 & 0.027225 & 2847310 & 0.279246 & 0.001451 \\
\hline Down & LYN & 51 & 0.028956 & 2899660 & 0.294947 & 0.007843 \\
\hline Down & SGK1 & 49 & 0.024995 & 1409808 & 0.277394 & 0 \\
\hline Down & STAT5A & 48 & 0.023474 & 2127412 & 0.272419 & 0 \\
\hline Down & MYL12A & 47 & 0.03358 & 2898132 & 0.316471 & 0.00555 \\
\hline Down & WAS & 47 & 0.020867 & 1130420 & 0.268207 & 0.002775 \\
\hline Down & DNAJB6 & 46 & 0.026431 & 1500174 & 0.289411 & 0.01256 \\
\hline Down & TSC22D4 & 44 & 0.020359 & 2077920 & 0.244808 & 0 \\
\hline Down & PYCARD & 44 & 0.020627 & 1299182 & 0.263235 & 0 \\
\hline Down & EZR & 43 & 0.026535 & 2455568 & 0.293219 & 0.016611 \\
\hline Down & CCND1 & 42 & 0.018913 & 907250 & 0.275293 & 0.00813 \\
\hline Down & PTPN6 & 41 & 0.020258 & 1324754 & 0.279451 & 0.010976 \\
\hline Down & SYK & 40 & 0.018577 & 1676464 & 0.275517 & 0.021795 \\
\hline Down & DAB2 & 40 & 0.017683 & 3025304 & 0.271305 & 0 \\
\hline Down & ITSN1 & 38 & 0.014931 & 1398302 & 0.26233 & 0.001422 \\
\hline Down & ITGB5 & 37 & 0.017897 & 1383852 & 0.265732 & 0.001502 \\
\hline Down & CSK & 37 & 0.014855 & 1938548 & 0.261566 & 0.001502 \\
\hline Down & SERTAD1 & 36 & 0.024153 & 963806 & 0.279349 & 0.001587 \\
\hline Down & PTBP1 & 36 & 0.014763 & 2873566 & 0.274575 & 0 \\
\hline Down & ITGB1 & 36 & 0.022917 & 1918002 & 0.282345 & 0.004762 \\
\hline Down & RPS27 & 35 & 0.017116 & 1486142 & 0.281954 & 0.001681 \\
\hline
\end{tabular}


medRxiv preprint doi: https://doi.org/10.1101/2020.12.21.20248688; this version posted December 24, 2020. The copyright holder for this preprint (which was not certified by peer review) is the author/funder, who has granted medRxiv a license to display the preprint in perpetuity. All rights reserved. No reuse allowed without permission.

\begin{tabular}{|c|c|c|c|c|c|c|}
\hline Down & DOCK2 & 35 & 0.014995 & 2204944 & 0.258007 & 0 \\
\hline Down & ATXN3 & 34 & 0.024882 & 1110164 & 0.27614 & 0.003565 \\
\hline Down & CLIC1 & 32 & 0.013199 & 1852884 & 0.275666 & 0 \\
\hline Down & PHGDH & 31 & 0.014535 & 2331562 & 0.278406 & 0.002151 \\
\hline Down & SLC9A3R1 & 27 & 0.009181 & 445502 & 0.257246 & 0.019943 \\
\hline Down & TAP1 & 27 & 0.012997 & 1909902 & 0.231709 & 0 \\
\hline Down & RAB3IL1 & 27 & 0.011749 & 1816470 & 0.236667 & 0 \\
\hline Down & RASSF2 & 26 & 0.009885 & 838798 & 0.260806 & 0.018462 \\
\hline Down & FERMT3 & 25 & 0.012868 & 1434660 & 0.262895 & 0 \\
\hline Down & SEC61A1 & 25 & 0.012969 & 1088072 & 0.284264 & 0.006667 \\
\hline Down & VAMP3 & 25 & 0.008816 & 1572520 & 0.242263 & 0 \\
\hline Down & FGFR3 & 25 & 0.011955 & 1002686 & 0.253719 & 0 \\
\hline Down & MEGF10 & 24 & 0.013349 & 1078384 & 0.215592 & 0 \\
\hline Down & MAPKAPK3 & 24 & 0.010404 & 453004 & 0.289466 & 0.003623 \\
\hline Down & FBLN1 & 24 & 0.012476 & 1085908 & 0.257333 & 0 \\
\hline Down & APOE & 24 & 0.014171 & 988930 & 0.255012 & 0.007246 \\
\hline Down & UGGT1 & 23 & 0.011922 & 1500648 & 0.232574 & 0 \\
\hline Down & FAM107A & 23 & 0.008353 & 1041506 & 0.25448 & 0 \\
\hline Down & PRMT2 & 23 & 0.01039 & 1156514 & 0.24165 & 0 \\
\hline Down & CRYAB & 23 & 0.009386 & 383150 & 0.277218 & 0.023715 \\
\hline Down & ARHGEF6 & 22 & 0.007755 & 852056 & 0.254502 & 0 \\
\hline Down & PLOD3 & 22 & 0.011436 & 1746324 & 0.270824 & 0 \\
\hline Down & MSN & 22 & 0.01101 & 799588 & 0.282528 & 0.038961 \\
\hline Down & MKNK2 & 22 & 0.009468 & 812402 & 0.23781 & 0 \\
\hline Down & AHNAK & 21 & 0.005477 & 1281178 & 0.279758 & 0 \\
\hline Down & DFFA & 21 & 0.00776 & 522714 & 0.286317 & 0.02381 \\
\hline Down & PDE9A & 20 & 0.00834 & 698368 & 0.242417 & 0 \\
\hline Down & INPPL1 & 20 & 0.005406 & 894622 & 0.254502 & 0 \\
\hline Down & GBP2 & 20 & 0.008984 & 436408 & 0.252377 & 0.005263 \\
\hline Down & СЕВРВ & 20 & 0.012045 & 996912 & 0.261342 & 0.052632 \\
\hline Down & CD44 & 20 & 0.008011 & 410804 & 0.267643 & 0.026316 \\
\hline Down & PALLD & 19 & 0.004224 & 675612 & 0.241726 & 0 \\
\hline Down & ABCA1 & 19 & 0.00924 & 819436 & 0.248474 & 0 \\
\hline Down & VASN & 18 & 0.005701 & 668270 & 0.234108 & 0 \\
\hline Down & PARVG & 18 & 0.008157 & 848264 & 0.244867 & 0 \\
\hline Down & HIPK2 & 18 & 0.007062 & 683068 & 0.252439 & 0 \\
\hline Down & RAI14 & 18 & 0.002903 & 765796 & 0.252669 & 0 \\
\hline Down & HMG20B & 18 & 0.006404 & 409650 & 0.266987 & 0.019608 \\
\hline Down & PRDX6 & 18 & 0.005221 & 1081120 & 0.258422 & 0 \\
\hline Down & LAPTM5 & 18 & 0.008887 & 710260 & 0.225964 & 0 \\
\hline Down & HSPA1A & 18 & 0.006718 & 665644 & 0.249306 & 0.013072 \\
\hline Down & CARHSP1 & 17 & 0.006023 & 525748 & 0.251566 & 0 \\
\hline Down & ITGB2 & 17 & 0.007497 & 499952 & 0.265225 & 0.014706 \\
\hline
\end{tabular}


medRxiv preprint doi: https://doi.org/10.1101/2020.12.21.20248688; this version posted December 24, 2020. The copyright holder for this preprint (which was not certified by peer review) is the author/funder, who has granted medRxiv a license to display the preprint in perpetuity. All rights reserved. No reuse allowed without permission.

\begin{tabular}{|c|c|c|c|c|c|c|}
\hline Down & CD14 & 17 & 0.007843 & 534520 & 0.229808 & 0 \\
\hline Down & SYTL4 & 16 & 0.006597 & 627446 & 0.23038 & 0 \\
\hline Down & VAMP8 & 16 & 0.006795 & 782382 & 0.250863 & 0 \\
\hline Down & CEBPA & 16 & 0.00441 & 191576 & 0.239207 & 0.075 \\
\hline Down & PDCD4 & 15 & 0.005464 & 350722 & 0.246986 & 0 \\
\hline Down & MYO10 & 15 & 0.004772 & 295330 & 0.256017 & 0.009524 \\
\hline Down & MYBPC1 & 15 & 0.004639 & 259024 & 0.233553 & 0.038095 \\
\hline Down & ITGB4 & 15 & 0.005303 & 705034 & 0.241841 & 0 \\
\hline Down & ALOX5 & 15 & 0.005415 & 298364 & 0.243209 & 0 \\
\hline Down & TNS3 & 14 & 0.00206 & 322150 & 0.240451 & 0 \\
\hline Down & ANGPTL4 & 14 & 0.00522 & 234096 & 0.258051 & 0 \\
\hline Down & TRIB1 & 14 & 0.006864 & 291486 & 0.222311 & 0 \\
\hline Down & PLOD1 & 14 & 0.002897 & 716168 & 0.239978 & 0 \\
\hline Down & RHOC & 14 & 0.003801 & 635342 & 0.232645 & 0 \\
\hline Down & ADRB2 & 14 & 0.005025 & 304860 & 0.253172 & 0 \\
\hline Down & MOB3C & 13 & 0.00534 & 250762 & 0.234072 & 0 \\
\hline Down & CYBRD1 & 13 & 0.002396 & 628468 & 0.225347 & 0 \\
\hline Down & HERC5 & 13 & 0.005161 & 435588 & 0.239601 & 0 \\
\hline Down & WASF2 & 13 & 0.003836 & 257378 & 0.252941 & 0.012821 \\
\hline Down & TBL1X & 13 & 0.003935 & 391598 & 0.241268 & 0 \\
\hline Down & ITPKB & 13 & 0.00559 & 459996 & 0.230033 & 0 \\
\hline Down & C1QA & 13 & 0.004112 & 399198 & 0.223287 & 0.038462 \\
\hline Down & ADD3 & 13 & 0.00828 & 504508 & 0.294919 & 0 \\
\hline Down & HAVCR2 & 12 & 0.001633 & 96970 & 0.230921 & 0.075758 \\
\hline Down & LEMD3 & 12 & 0.006001 & 323982 & 0.247447 & 0 \\
\hline Down & SIPA1 & 12 & 0.003772 & 346372 & 0.265432 & 0 \\
\hline Down & RRBP1 & 12 & 0.003079 & 322094 & 0.243151 & 0 \\
\hline Down & MT2A & 12 & 0.005586 & 258386 & 0.203222 & 0 \\
\hline Down & FGR & 12 & 0.003722 & 318494 & 0.266638 & 0.090909 \\
\hline Down & DDIT4L & 11 & 0.004247 & 492198 & 0.22345 & 0 \\
\hline Down & HSPB8 & 11 & 0.004255 & 238968 & 0.267268 & 0.127273 \\
\hline Down & MYOT & 11 & 0.003913 & 254532 & 0.216971 & 0 \\
\hline Down & SELENBP1 & 11 & 0.003304 & 321380 & 0.234431 & 0 \\
\hline Down & MAPK4 & 11 & 0.004295 & 454710 & 0.236648 & 0 \\
\hline Down & GSTM3 & 11 & 0.00351 & 432762 & 0.234323 & 0 \\
\hline Down & DDR1 & 11 & 0.003995 & 307240 & 0.227972 & 0 \\
\hline Down & LSM11 & 10 & 0.003484 & 236152 & 0.221827 & 0 \\
\hline Down & NECAP2 & 10 & 0.002181 & 292664 & 0.253867 & 0 \\
\hline Down & EHD4 & 10 & 0.003286 & 462476 & 0.241077 & 0 \\
\hline Down & FERMT2 & 10 & 0.003104 & 240800 & 0.251503 & 0 \\
\hline Down & FAM189A2 & 10 & 0.003552 & 215364 & 0.246927 & 0 \\
\hline Down & SPP1 & 10 & 0.004672 & 230708 & 0.243189 & 0 \\
\hline Down & S100A1 & 10 & 0.003843 & 339426 & 0.244514 & 0 \\
\hline
\end{tabular}


medRxiv preprint doi: https://doi.org/10.1101/2020.12.21.20248688; this version posted December 24, 2020. The copyright holder for this preprint (which was not certified by peer review) is the author/funder, who has granted medRxiv a license to display the preprint in perpetuity. All rights reserved. No reuse allowed without permission.

\begin{tabular}{|c|c|c|c|c|c|c|}
\hline Down & RAC2 & 10 & 0.004626 & 231210 & 0.252147 & 0 \\
\hline Down & LAMP2 & 10 & 0.002846 & 149214 & 0.245004 & 0 \\
\hline Down & HLA-DQA1 & 10 & 0.003467 & 284172 & 0.241325 & 0 \\
\hline Down & SERPINA3 & 10 & 0.005255 & 351298 & 0.218759 & 0 \\
\hline Down & DDIT4 & 9 & 0.002143 & 249074 & 0.241363 & 0 \\
\hline Down & VAMP5 & 9 & 0.002253 & 536658 & 0.186968 & 0 \\
\hline Down & PLIN3 & 9 & 0.005229 & 256176 & 0.235953 & 0 \\
\hline Down & FARP1 & 9 & 0.003218 & 462100 & 0.21829 & 0 \\
\hline Down & SLC16A3 & 9 & 0.002082 & 191722 & 0.226484 & 0 \\
\hline Down & PLEK & 9 & 0.002846 & 155246 & 0.23994 & 0 \\
\hline Down & PC & 9 & 0.003632 & 350054 & 0.233463 & 0 \\
\hline Down & FOXO4 & 9 & 0.00285 & 254500 & 0.243035 & 0 \\
\hline Down & SP110 & 9 & 0.003327 & 124378 & 0.256706 & 0 \\
\hline Down & HCLS1 & 9 & 0.002394 & 123870 & 0.258291 & 0.055556 \\
\hline Down & GPX1 & 9 & 0.001298 & 152998 & 0.219214 & 0 \\
\hline Down & DBI & 9 & 0.003463 & 405680 & 0.222214 & 0 \\
\hline Down & CD74 & 9 & 0.00277 & 152896 & 0.219798 & 0 \\
\hline Down & RHPN2 & 8 & 0.002341 & 238122 & 0.212161 & 0 \\
\hline Down & ANTXR1 & 8 & 0.002541 & 393834 & 0.220784 & 0 \\
\hline Down & CSRNP1 & 8 & 0.003321 & 289950 & 0.21045 & 0 \\
\hline Down & KANK1 & 8 & 0.001946 & 154546 & 0.241497 & 0.035714 \\
\hline Down & RHOBTB3 & 8 & 0.003572 & 135278 & 0.210291 & 0 \\
\hline Down & ADAM17 & 8 & 0.002632 & 105124 & 0.241611 & 0.035714 \\
\hline Down & TROVE2 & 8 & 8.35E-04 & 186946 & 0.249143 & 0 \\
\hline Down & PLXNB1 & 8 & 0.003449 & 511002 & 0.213586 & 0 \\
\hline Down & OLR1 & 8 & 0.001344 & 264214 & 0.210843 & 0 \\
\hline Down & LPP & 8 & 0.005157 & 362612 & 0.215851 & 0 \\
\hline Down & IRF8 & 8 & 0.003931 & 211768 & 0.216065 & 0 \\
\hline Down & DTNA & 8 & 0.002923 & 246614 & 0.220084 & 0 \\
\hline Down & CTSC & 8 & 0.002792 & 321900 & 0.205202 & 0 \\
\hline Down & SDC4 & 7 & 0.002524 & 136286 & 0.212797 & 0 \\
\hline Down & NTRK2 & 7 & 0.002703 & 423896 & 0.235535 & 0 \\
\hline Down & CYBA & 7 & 0.002895 & 196730 & 0.235589 & 0 \\
\hline Down & CEBPD & 7 & $1.36 \mathrm{E}-05$ & 508 & 0.210682 & 0.47619 \\
\hline Down & AXL & 7 & 0.001037 & 248436 & 0.242475 & 0 \\
\hline Down & EML3 & 6 & 0.001231 & 80812 & 0.243714 & 0.066667 \\
\hline Down & BRI3BP & 6 & 0.001275 & 166978 & 0.232238 & 0 \\
\hline Down & SRGAP1 & 6 & 0.001078 & 109132 & 0.236483 & 0 \\
\hline Down & PSAT1 & 6 & 0.002172 & 224000 & 0.229964 & 0 \\
\hline Down & RAB31 & 6 & 0.001583 & 254760 & 0.208681 & 0 \\
\hline Down & PAPOLA & 6 & 0.002134 & 242390 & 0.220322 & 0 \\
\hline Down & MYOM1 & 6 & 0.001242 & 67972 & 0.229273 & 0.133333 \\
\hline Down & TIMP1 & 6 & 0.00266 & 138732 & 0.181715 & 0 \\
\hline
\end{tabular}


medRxiv preprint doi: https://doi.org/10.1101/2020.12.21.20248688; this version posted December 24, 2020. The copyright holder for this preprint (which was not certified by peer review) is the author/funder, who has granted medRxiv a license to display the preprint in perpetuity. All rights reserved. No reuse allowed without permission.

\begin{tabular}{|c|c|c|c|c|c|c|}
\hline Down & TGFB3 & 6 & 0.001972 & 111928 & 0.188259 & 0 \\
\hline Down & CD37 & 6 & 0.002032 & 192264 & 0.238311 & 0.066667 \\
\hline Down & BST2 & 6 & 0.00216 & 203622 & 0.209713 & 0 \\
\hline Down & SFMBT2 & 6 & 1 & 30 & 1 & 0 \\
\hline Down & СMTM3 & 5 & 0.001583 & 84622 & 0.208112 & 0 \\
\hline Down & UNC93B1 & 5 & 0.002144 & 228958 & 0.198569 & 0 \\
\hline Down & FEM1C & 5 & 0.001371 & 136164 & 0.207081 & 0 \\
\hline Down & ASAP3 & 5 & 0.001235 & 126860 & 0.207828 & 0 \\
\hline Down & TMEM51 & 5 & 0.001492 & 130816 & 0.213467 & 0 \\
\hline Down & RHOQ & 5 & 0.001021 & 46560 & 0.22386 & 0 \\
\hline Down & PIEZO1 & 5 & $8.90 \mathrm{E}-04$ & 102684 & 0.192376 & 0 \\
\hline Down & TNFSF14 & 5 & 0.002099 & 184096 & 0.190479 & 0 \\
\hline Down & AKR1C3 & 5 & 0.002619 & 152436 & 0.207899 & 0 \\
\hline Down & HIST2H2AA3 & 5 & 0.001021 & 123888 & 0.242533 & 0 \\
\hline Down & WFS1 & 5 & $9.55 \mathrm{E}-04$ & 112242 & 0.226048 & 0 \\
\hline Down & TYROBP & 5 & 0.001965 & 153540 & 0.216126 & 0.1 \\
\hline Down & RGS1 & 5 & 0.001423 & 133108 & 0.228006 & 0 \\
\hline Down & PTH1R & 5 & $2.22 \mathrm{E}-04$ & 56212 & 0.237921 & 0 \\
\hline Down & HTRA1 & 5 & 0.001778 & 145232 & 0.240318 & 0 \\
\hline Down & PPP1R3C & 5 & 0.001456 & 114348 & 0.214909 & 0 \\
\hline Down & IL13RA1 & 5 & 0.001975 & 116798 & 0.200948 & 0 \\
\hline Down & HMOX1 & 5 & 0.001107 & 112536 & 0.212827 & 0 \\
\hline Down & FCGR1A & 5 & 7.02E-04 & 41656 & 0.223516 & 0.1 \\
\hline Down & CSF2RA & 5 & 0.001279 & 206560 & 0.215562 & 0 \\
\hline Down & CNN3 & 5 & 4.03E-04 & 59632 & 0.233714 & 0 \\
\hline Down & TPP1 & 5 & 0.001439 & 104628 & 0.240716 & 0 \\
\hline Down & ALDH7A1 & 5 & 0.00266 & 181084 & 0.224717 & 0 \\
\hline Down & ARHGDIB & 5 & $8.49 \mathrm{E}-04$ & 29570 & 0.216802 & 0 \\
\hline Down & OTOS & 4 & 0.005385 & 231968 & 0.205133 & 0 \\
\hline Down & CGNL1 & 4 & $6.69 \mathrm{E}-04$ & 57352 & 0.217714 & 0 \\
\hline Down & XAF1 & 4 & 0.001344 & 75474 & 0.224783 & 0 \\
\hline Down & SOX8 & 4 & 0.001965 & 120444 & 0.192001 & 0 \\
\hline Down & BAZ2B & 4 & 0.001341 & 114986 & 0.189029 & 0 \\
\hline Down & SLC1A3 & 4 & $5.21 \mathrm{E}-04$ & 65480 & 0.224222 & 0 \\
\hline Down & NDP & 4 & 0.001352 & 114228 & 0.19042 & 0 \\
\hline Down & MT1X & 4 & 7.42E-04 & 59832 & 0.210305 & 0 \\
\hline Down & MT1M & 4 & 0.001337 & 65806 & 0.204091 & 0 \\
\hline Down & MT1A & 4 & 0.001623 & 109652 & 0.234973 & 0 \\
\hline Down & MSX1 & 4 & 8.37E-04 & 102558 & 0.214743 & 0 \\
\hline Down & LRP4 & 4 & 0.001965 & 100110 & 0.200552 & 0 \\
\hline Down & HOXB6 & 4 & $1.79 \mathrm{E}-04$ & 22804 & 0.223925 & 0 \\
\hline Down & GJA1 & 4 & 0.001084 & 44798 & 0.221891 & 0.166667 \\
\hline Down & EMP1 & 4 & 0.001398 & 132346 & 0.16694 & 0 \\
\hline
\end{tabular}


medRxiv preprint doi: https://doi.org/10.1101/2020.12.21.20248688; this version posted December 24, 2020. The copyright holder for this preprint (which was not certified by peer review) is the author/funder, who has granted medRxiv a license to display the preprint in perpetuity. All rights reserved. No reuse allowed without permission.

\begin{tabular}{|c|c|c|c|c|c|c|}
\hline Down & NQO1 & 4 & 0.00136 & 97788 & 0.237237 & 0 \\
\hline Down & CSRP1 & 4 & 2.79E-04 & 67996 & 0.252481 & 0 \\
\hline Down & CSF1R & 4 & $1.98 \mathrm{E}-04$ & 35642 & 0.23261 & 0 \\
\hline Down & CD86 & 4 & 0.00134 & 79656 & 0.179863 & 0 \\
\hline Down & C1QB & 4 & $9.27 \mathrm{E}-04$ & 62438 & 0.212649 & 0.166667 \\
\hline Down & BGN & 4 & $7.30 \mathrm{E}-04$ & 28828 & 0.187842 & 0 \\
\hline Down & RHOG & 4 & 0.001341 & 192572 & 0.184739 & 0 \\
\hline Down & ACACB & 4 & 0.001192 & 78772 & 0.229394 & 0 \\
\hline Down & NUPR1 & 4 & 1 & 12 & 1 & 0 \\
\hline Down & SLC16A9 & 3 & $1.55 \mathrm{E}-05$ & 354 & 0.209253 & 0 \\
\hline Down & GLIS3 & 3 & 0.00131 & 87034 & 0.190265 & 0 \\
\hline Down & S100A16 & 3 & 7.06E-04 & 96488 & 0.232734 & 0 \\
\hline Down & СМТМ7 & 3 & $8.88 \mathrm{E}-04$ & 101080 & 0.17962 & 0 \\
\hline Down & SCIN & 3 & $1.30 \mathrm{E}-05$ & 2728 & 0.225897 & 0 \\
\hline Down & GPT2 & 3 & $6.90 \mathrm{E}-04$ & 70280 & 0.1717 & 0 \\
\hline Down & SLC25A18 & 3 & $6.79 \mathrm{E}-04$ & 30078 & 0.207208 & 0 \\
\hline Down & EFHD1 & 3 & 0.00131 & 91334 & 0.168991 & 0 \\
\hline Down & DCLRE1C & 3 & 0.00131 & 62046 & 0.188492 & 0 \\
\hline Down & ZNF462 & 3 & 0.00131 & 101282 & 0.20356 & 0 \\
\hline Down & ADAP2 & 3 & 3.64E-04 & 40040 & 0.217357 & 0 \\
\hline Down & RIN2 & 3 & 7.47E-05 & 15764 & 0.237181 & 0 \\
\hline Down & SLC15A3 & 3 & 7.35E-05 & 7620 & 0.189193 & 0 \\
\hline Down & LY96 & 3 & $6.89 \mathrm{E}-04$ & 23444 & 0.189099 & 0 \\
\hline Down & STAB1 & 3 & 7.03E-04 & 65946 & 0.230572 & 0 \\
\hline Down & RGS10 & 3 & 7.09E-04 & 40278 & 0.218759 & 0 \\
\hline Down & PRCP & 3 & 0.00131 & 81590 & 0.208995 & 0 \\
\hline Down & PLCD1 & 3 & $9.07 \mathrm{E}-05$ & 13232 & 0.21259 & 0 \\
\hline Down & МOBP & 3 & 7.94E-05 & 11894 & 0.19274 & 0 \\
\hline Down & LCP1 & 3 & $6.79 \mathrm{E}-04$ & 74352 & 0.231235 & 0 \\
\hline Down & IFNGR1 & 3 & $6.79 \mathrm{E}-04$ & 54930 & 0.214622 & 0 \\
\hline Down & HLA-DMB & 3 & 0.00131 & 109642 & 0.208268 & 0 \\
\hline Down & GNA12 & 3 & 0.00131 & 88106 & 0.150855 & 0 \\
\hline Down & FCER1G & 3 & $8.11 \mathrm{E}-05$ & 3528 & 0.22104 & 0 \\
\hline Down & EYA2 & 3 & 7.67E-04 & 53910 & 0.182345 & 0 \\
\hline Down & EMP3 & 3 & 0.001318 & 98464 & 0.198028 & 0 \\
\hline Down & PTTG1IP & 3 & 0.00131 & 95918 & 0.163131 & 0 \\
\hline Down & C1QC & 3 & $3.33 \mathrm{E}-05$ & 4288 & 0.201186 & 0.666667 \\
\hline Down & ZFHX3 & 3 & $8.00 \mathrm{E}-04$ & 63208 & 0.232009 & 0 \\
\hline Down & APOC2 & 3 & $2.67 \mathrm{E}-05$ & 4132 & 0.214246 & 0.666667 \\
\hline Down & APOC1 & 3 & $1.27 \mathrm{E}-04$ & 11920 & 0.203168 & 0 \\
\hline Down & AIF1 & 3 & 0.00131 & 137650 & 0.176944 & 0 \\
\hline Down & TRIM47 & 3 & 1 & 6 & 1 & 0 \\
\hline Down & RAPGEF3 & 3 & 1 & 6 & 1 & 0 \\
\hline
\end{tabular}


medRxiv preprint doi: https://doi.org/10.1101/2020.12.21.20248688; this version posted December 24, 2020. The copyright holder for this preprint (which was not certified by peer review) is the author/funder, who has granted medRxiv a license to display the preprint in perpetuity. All rights reserved. No reuse allowed without permission.

\begin{tabular}{|c|c|c|c|c|c|}
\hline Down & RANBP3L & 2 & $2.96 \mathrm{E}-05$ & 4290 & 0.20283 \\
\hline Down & NACC2 & 2 & $6.55 \mathrm{E}-04$ & 38996 & 0.176047 \\
\hline Down & EMID1 & 2 & $6.55 \mathrm{E}-04$ & 60420 & 0.225497 \\
\hline Down & ACSS1 & 2 & 0.003271 & 137820 & 0.145624 \\
\hline Down & TMBIM1 & 2 & $6.55 \mathrm{E}-04$ & 21546 & 0.184739 \\
\hline Down & PLEKHB1 & 2 & $6.55 \mathrm{E}-04$ & 18776 & 0.191267 \\
\hline Down & SYNJ2BP & 2 & $4.84 \mathrm{E}-05$ & 7940 & 0.207631 \\
\hline Down & APBB1IP & 2 & $2.17 \mathrm{E}-04$ & 18666 & 0.228535 \\
\hline Down & ACSBG1 & 2 & $5.44 \mathrm{E}-05$ & 3048 & 0.195354 \\
\hline Down & LILRB1 & 2 & $6.55 \mathrm{E}-04$ & 92454 & 0.207377 \\
\hline Down & CERS1 & 2 & $6.48 \mathrm{E}-05$ & 8282 & 0.202911 \\
\hline Down & EBI3 & 2 & $1.48 \mathrm{E}-04$ & 21114 & 0.211207 \\
\hline Down & FCGBP & 2 & $2.07 \mathrm{E}-04$ & 13900 & 0.200921 \\
\hline Down & ALDH4A1 & 2 & $6.55 \mathrm{E}-04$ & 27572 & 0.112828 \\
\hline Down & BCAS1 & 2 & $7.29 \mathrm{E}-06$ & 1156 & 0.191159 \\
\hline Down & VEGFB & 2 & $6.55 \mathrm{E}-04$ & 77838 & 0.176148 \\
\hline Down & TST & 2 & $6.55 \mathrm{E}-04$ & 27572 & 0.112828 \\
\hline Down & SLC2A5 & 2 & $6.55 \mathrm{E}-04$ & 33090 & 0.206731 \\
\hline Down & NINJ1 & 2 & $6.37 \mathrm{E}-05$ & 3454 & 0.205313 \\
\hline Down & MAOA & 2 & $1.98 \mathrm{E}-04$ & 2692 & 0.226517 \\
\hline Down & KCNMA1 & 2 & $6.55 \mathrm{E}-04$ & 60118 & 0.19263 \\
\hline Down & HLA-DRA & 2 & $6.55 \mathrm{E}-04$ & 35690 & 0.180224 \\
\hline Down & GYPC & 2 & $1.99 \mathrm{E}-05$ & 2214 & 0.189533 \\
\hline Down & CSF3R & 2 & $9.84 \mathrm{E}-06$ & 1098 & 0.215973 \\
\hline Down & ABCB7 & 2 & $6.55 \mathrm{E}-04$ & 37292 & 0.227615 \\
\hline Down & MS4A7 & 2 & 1 & 2 & 1 \\
\hline Down & CD151 & 2 & 1 & 2 & 1 \\
\hline Down & HCST & 2 & 1 & 2 & 1 \\
\hline Down & IFITM2 & 2 & 1 & 2 & 1 \\
\hline Down & HLA-DMA & 2 & 1 & 2 & 1 \\
\hline Down & EMX2 & 2 & 1 & 2 & 1 \\
\hline Down & CYP2J2 & 2 & 1 & 2 & 1 \\
\hline Down & SLC9A9 & 1 & 0 & 0 & 0.187473 \\
\hline Down & NWD1 & 1 & 0 & 0 & 0.18706 \\
\hline Down & RFTN2 & 1 & 0 & 0 & 0.166494 \\
\hline Down & SIGLEC10 & 1 & 0 & 0 & 0.21843 \\
\hline Down & NPL & 1 & 0 & 0 & 0.180587 \\
\hline Down & LHPP & 1 & 0 & 0 & 0.159835 \\
\hline Down & COL20A1 & 1 & 0 & 0 & 0.197746 \\
\hline Down & NDRG2 & 1 & 0 & 0 & 0.221168 \\
\hline Down & IL17RB & 1 & 0 & 0 & 0.172097 \\
\hline Down & SH3TC1 & 1 & 0 & 0 & 0.188399 \\
\hline Down & ARRDC2 & 1 & 0 & 0 & 0.14806 \\
\hline
\end{tabular}




\begin{tabular}{|c|c|c|c|c|c|c|}
\hline Down & TMC6 & 1 & 0 & 0 & 0.187473 & 0 \\
\hline Down & $\mathrm{CD} 300 \mathrm{~A}$ & 1 & 0 & 0 & 0.174089 & 0 \\
\hline Down & ALDH1L1 & 1 & 0 & 0 & 0.165081 & 0 \\
\hline Down & MERTK & 1 & 0 & 0 & 0.222928 & 0 \\
\hline Down & GPNMB & 1 & 0 & 0 & 0.200249 & 0 \\
\hline Down & CHST3 & 1 & 0 & 0 & 0.188027 & 0 \\
\hline Down & LY86 & 1 & 0 & 0 & 0.158367 & 0 \\
\hline Down & GPR37L1 & 1 & 0 & 0 & 0.172877 & 0 \\
\hline Down & BBOX1 & 1 & 0 & 0 & 0.174208 & 0 \\
\hline Down & PLA2G7 & 1 & 0 & 0 & 0.221168 & 0 \\
\hline Down & LAT2 & 1 & 0 & 0 & 0.163647 & 0 \\
\hline Down & $\mathrm{UCP} 2$ & 1 & 0 & 0 & 0.167453 & 0 \\
\hline Down & SLC7A2 & 1 & 0 & 0 & 0.212279 & 0 \\
\hline Down & RYR3 & 1 & 0 & 0 & 0.178423 & 0 \\
\hline Down & RARRES3 & 1 & 0 & 0 & 0.192218 & 0 \\
\hline Down & PMP2 & 1 & 0 & 0 & 0.212842 & 0 \\
\hline Down & OAS2 & 1 & 0 & 0 & 0.188306 & 0 \\
\hline Down & MYT1 & 1 & 0 & 0 & 0.197108 & 0 \\
\hline Down & ITGAX & 1 & 0 & 0 & 0.209641 & 0 \\
\hline Down & HLA-DPA1 & 1 & 0 & 0 & 0.180203 & 0 \\
\hline Down & HLA-DOA & 1 & 0 & 0 & 0.194918 & 0 \\
\hline Down & FCGRT & 1 & 0 & 0 & 0.158367 & 0 \\
\hline Down & LPAR1 & 1 & 0 & 0 & 0.173594 & 0 \\
\hline Down & CTSH & 1 & 0 & 0 & 0.227547 & 0 \\
\hline Down & TSPO & 1 & 0 & 0 & 0.142152 & 0 \\
\hline Down & ALOX5AP & 1 & 0 & 0 & 0.216111 & 0 \\
\hline Down & PLIN2 & 1 & 0 & 0 & 0.18812 & 0 \\
\hline
\end{tabular}

Table 7 miRNA - target gene interaction table

\begin{tabular}{|c|c|c|c|c|c|c|c|}
\hline $\begin{array}{c}\text { Regulatio } \\
\mathbf{n}\end{array}$ & $\begin{array}{l}\text { Target } \\
\text { Genes }\end{array}$ & $\begin{array}{c}\text { Degre } \\
\text { e }\end{array}$ & $\begin{array}{c}\text { MicroRN } \\
\text { A }\end{array}$ & $\begin{array}{c}\text { Regulatio } \\
\mathbf{n}\end{array}$ & $\begin{array}{l}\text { Target } \\
\text { Genes }\end{array}$ & $\begin{array}{c}\text { Degre } \\
\text { e }\end{array}$ & $\begin{array}{c}\text { MicroRN } \\
\text { A }\end{array}$ \\
\hline Up & TUBB2A & 183 & $\begin{array}{c}\text { hsa-mir- } \\
\text { 5681a }\end{array}$ & Down & CCND1 & 197 & $\begin{array}{c}\text { hsa-mir- } \\
3973\end{array}$ \\
\hline $\mathrm{Up}$ & YWHAZ & 156 & $\begin{array}{l}\text { hsa-mir- } \\
3189-5 p\end{array}$ & Down & MKNK2 & 153 & $\begin{array}{l}\text { hsa-mir- } \\
6515-5 p\end{array}$ \\
\hline Up & MAP3K9 & 121 & $\begin{array}{l}\text { hsa-mir- } \\
6791-3 p\end{array}$ & Down & CDKN1A & 131 & $\begin{array}{l}\text { hsa-mir- } \\
6886-3 p\end{array}$ \\
\hline $\mathrm{Up}$ & PGM2L1 & 117 & $\begin{array}{l}\text { hsa-mir- } \\
6764-3 p\end{array}$ & Down & SYNJ2BP & 120 & $\begin{array}{l}\text { hsa-mir- } \\
4659 a-3 p\end{array}$ \\
\hline
\end{tabular}




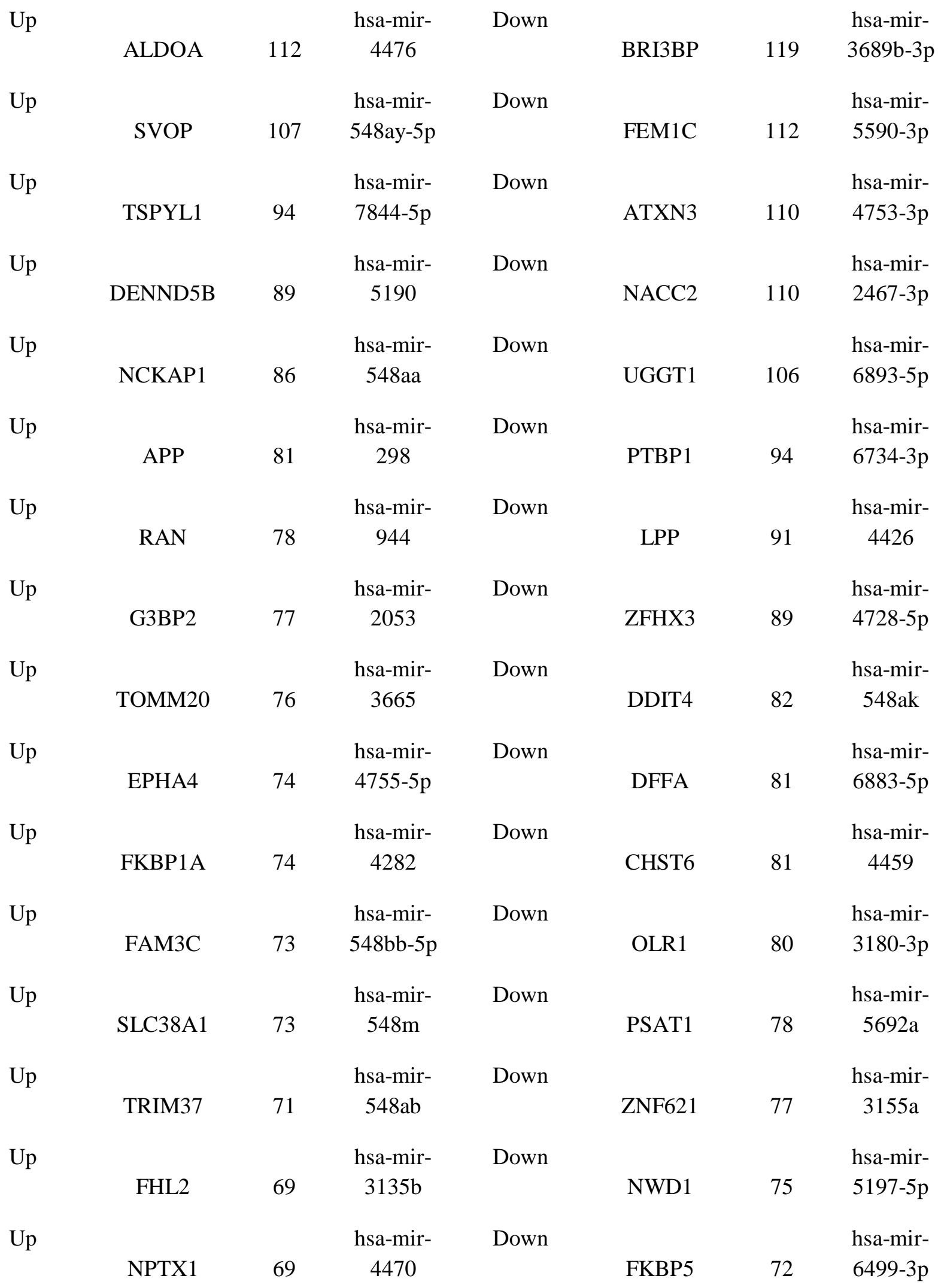




\begin{tabular}{|c|c|c|c|c|c|c|c|}
\hline Up & MAP1B & 68 & $\begin{array}{l}\text { hsa-mir- } \\
4499\end{array}$ & Down & TRIB1 & 72 & $\begin{array}{l}\text { hsa-mir- } \\
6779-5 p\end{array}$ \\
\hline Up & SYNGR1 & 64 & $\begin{array}{l}\text { hsa-mir- } \\
5189-5 p\end{array}$ & Down & COX19 & 72 & $\begin{array}{l}\text { hsa-mir- } \\
6802-3 p\end{array}$ \\
\hline Up & KIAA0513 & 63 & $\begin{array}{l}\text { hsa-mir- } \\
548 x-5 p\end{array}$ & Down & SLC16A9 & 70 & $\begin{array}{l}\text { hsa-mir- } \\
2114-5 p\end{array}$ \\
\hline Up & RNF41 & 63 & $\begin{array}{c}\text { hsa-mir- } \\
1200\end{array}$ & Down & FZD9 & 69 & $\begin{array}{c}\text { hsa-mir- } \\
5700\end{array}$ \\
\hline Up & NT5DC3 & 63 & $\begin{array}{l}\text { hsa-mir- } \\
548 \mathrm{az}-3 \mathrm{p}\end{array}$ & Down & SLC5A3 & 68 & $\begin{array}{c}\text { hsa-mir- } \\
4465\end{array}$ \\
\hline Up & CAPRIN2 & 61 & $\begin{array}{c}\text { hsa-mir- } \\
3666\end{array}$ & Down & KCNN3 & 66 & $\begin{array}{c}\text { hsa-mir- } \\
5100\end{array}$ \\
\hline Up & CALM3 & 60 & $\begin{array}{l}\text { hsa-mir- } \\
6760-3 p\end{array}$ & Down & MYBPC1 & 65 & $\begin{array}{c}\text { hsa-mir- } \\
4424\end{array}$ \\
\hline Up & RBFOX2 & 59 & $\begin{array}{c}\text { hsa-mir- } \\
3133\end{array}$ & Down & LHFPL2 & 65 & $\begin{array}{r}\text { hsa-mir- } \\
1260 \mathrm{a}\end{array}$ \\
\hline $\mathrm{Up}$ & STMN1 & 58 & $\begin{array}{l}\text { hsa-mir- } \\
4433 a-3 p\end{array}$ & Down & PDCD4 & 65 & $\begin{array}{c}\text { hsa-mir- } \\
5697\end{array}$ \\
\hline Up & MAP2K4 & 58 & $\begin{array}{l}\text { hsa-mir- } \\
548 \text { as-5p }\end{array}$ & Down & ADAM17 & 64 & $\begin{array}{c}\text { hsa-mir- } \\
4505\end{array}$ \\
\hline Up & PRNP & 57 & $\begin{array}{l}\text { hsa-mir- } \\
520 \mathrm{~g}-3 \mathrm{p}\end{array}$ & Down & RHOBTB3 & 64 & $\begin{array}{l}\text { hsa-mir- } \\
892 c-3 p\end{array}$ \\
\hline Up & PLEKHB2 & 57 & $\begin{array}{c}\text { hsa-mir- } \\
4478\end{array}$ & Down & MYO10 & 58 & $\begin{array}{l}\text { hsa-mir- } \\
6792-3 p\end{array}$ \\
\hline Up & PSD3 & 56 & $\begin{array}{l}\text { hsa-mir- } \\
\text { 548ap-3p }\end{array}$ & Down & TROVE2 & 58 & $\begin{array}{l}\text { hsa-mir- } \\
2682-5 p\end{array}$ \\
\hline Up & ATP2A2 & 55 & $\begin{array}{l}\text { hsa-mir- } \\
548 \text { ar-3p }\end{array}$ & Down & SGK1 & 55 & $\begin{array}{l}\text { hsa-mir- } \\
5571-5 \mathrm{p}\end{array}$ \\
\hline Up & YWHAH & 55 & $\begin{array}{c}\text { hsa-mir- } \\
5087\end{array}$ & Down & PLXDC2 & 54 & $\begin{array}{c}\text { hsa-mir- } \\
3924\end{array}$ \\
\hline Up & FBXL2 & 55 & $\begin{array}{l}\text { hsa-mir- } \\
6843-3 p\end{array}$ & Down & MSN & 53 & $\begin{array}{c}\text { hsa-mir- } \\
\text { 3689d }\end{array}$ \\
\hline
\end{tabular}




\begin{tabular}{|c|c|c|c|c|c|c|}
\hline OCIAD1 & 55 & $\begin{array}{l}\text { hsa-mir- } \\
5095\end{array}$ & Down & LIPA & 52 & $\begin{array}{l}\text { hsa-mir- } \\
\text { 548ay-5p }\end{array}$ \\
\hline GOLGA8B & 54 & $\begin{array}{c}\text { hsa-mir- } \\
4319\end{array}$ & Down & NDRG1 & 52 & $\begin{array}{l}\text { hsa-mir- } \\
548 a z-3 p\end{array}$ \\
\hline PACSIN1 & 53 & $\begin{array}{l}\text { hsa-mir- } \\
\text { 3190-3p }\end{array}$ & Down & ACSBG1 & 51 & $\begin{array}{l}\text { hsa-mir- } \\
6511 a-5 p\end{array}$ \\
\hline MAP2K1 & 52 & $\begin{array}{c}\text { hsa-mir- } \\
\text { 548ak }\end{array}$ & Down & CARHSP1 & 51 & $\begin{array}{c}\text { hsa-mir- } \\
3909\end{array}$ \\
\hline SLC25A12 & 52 & $\begin{array}{c}\text { hsa-mir- } \\
1267\end{array}$ & Down & ITGB1 & 50 & $\begin{array}{l}\text { hsa-mir- } \\
\text { 3622b-5p }\end{array}$ \\
\hline PNMA2 & 52 & $\begin{array}{l}\text { hsa-mir- } \\
3681-5 p\end{array}$ & Down & SRGAP1 & 50 & $\begin{array}{c}\text { hsa-mir- } \\
4293\end{array}$ \\
\hline HPRT1 & 50 & $\begin{array}{l}\text { hsa-mir- } \\
548 y\end{array}$ & Down & RHOC & 49 & $\begin{array}{l}\text { hsa-mir- } \\
\text { 3124-3p }\end{array}$ \\
\hline GNB5 & 50 & $\begin{array}{l}\text { hsa-mir- } \\
520 \mathrm{e}\end{array}$ & Down & CD44 & 49 & $\begin{array}{l}\text { hsa-mir- } \\
\text { 4776-3p }\end{array}$ \\
\hline ATP6V1A & 48 & $\begin{array}{l}\text { hsa-mir- } \\
1273 \mathrm{~h}-5 \mathrm{p}\end{array}$ & Down & DNAJB6 & 48 & $\begin{array}{l}\text { hsa-mir- } \\
3152-3 p\end{array}$ \\
\hline PPP3R1 & 48 & $\begin{array}{l}\text { hsa-mir- } \\
548 \mathrm{ac}\end{array}$ & Down & ZCCHC24 & 48 & $\begin{array}{c}\text { hsa-mir- } \\
5096\end{array}$ \\
\hline UBE2N & 48 & $\begin{array}{l}\text { hsa-mir- } \\
6729-3 p\end{array}$ & Down & HMOX1 & 47 & $\begin{array}{l}\text { hsa-mir- } \\
\text { 5008-5p }\end{array}$ \\
\hline NPTXR & 48 & $\begin{array}{l}\text { hsa-mir- } \\
7156-3 p\end{array}$ & Down & $\begin{array}{c}\text { HIST2H2AA } \\
3\end{array}$ & 47 & $\begin{array}{l}\text { hsa-mir- } \\
2277-5 p\end{array}$ \\
\hline PRKCB & 47 & $\begin{array}{l}\text { hsa-mir- } \\
6074\end{array}$ & Down & TNFSF14 & 46 & $\begin{array}{c}\text { hsa-mir- } \\
4775\end{array}$ \\
\hline SCN2B & 46 & $\begin{array}{l}\text { hsa-mir- } \\
4770\end{array}$ & Down & PAPOLA & 46 & $\begin{array}{c}\text { hsa-mir- } \\
4327\end{array}$ \\
\hline MFSD6 & 45 & $\begin{array}{l}\text { hsa-mir- } \\
4722-3 p\end{array}$ & Down & SFMBT2 & 46 & $\begin{array}{l}\text { hsa-mir- } \\
4524 a-3 p\end{array}$ \\
\hline ENSA & 44 & $\begin{array}{l}\text { hsa-mir- } \\
4757-5 p\end{array}$ & Down & RFTN2 & 46 & $\begin{array}{l}\text { hsa-mir- } \\
\text { 30c-2-3p }\end{array}$ \\
\hline
\end{tabular}




\begin{tabular}{|c|c|c|c|c|c|c|}
\hline SV2B & 44 & $\begin{array}{l}\text { hsa-mir- } \\
4441\end{array}$ & Down & ACBD7 & 46 & $\begin{array}{c}\text { hsa-mir- } \\
5688\end{array}$ \\
\hline ATCAY & 42 & $\begin{array}{l}\text { hsa-mir- } \\
\text { 3934-5p }\end{array}$ & Down & SLC7A2 & 45 & $\begin{array}{l}\text { hsa-mir- } \\
6853-3 p\end{array}$ \\
\hline ATP2B1 & 41 & $\begin{array}{c}\text { hsa-mir- } \\
\text { 2276-5p }\end{array}$ & Down & PLIN3 & 45 & $\begin{array}{l}\text { hsa-mir- } \\
6759-5 p\end{array}$ \\
\hline AP1S1 & 41 & $\begin{array}{l}\text { hsa-mir- } \\
6499-3 p\end{array}$ & Down & CERS1 & 42 & $\begin{array}{c}\text { hsa-mir- } \\
3918\end{array}$ \\
\hline EPB41L1 & 40 & $\begin{array}{c}\text { hsa-mir- } \\
4510\end{array}$ & Down & CYBRD1 & 42 & $\begin{array}{l}\text { hsa-mir- } \\
138-1-3 p\end{array}$ \\
\hline TUBA1B & 40 & $\begin{array}{c}\text { hsa-mir- } \\
4643\end{array}$ & Down & EMP1 & 41 & $\begin{array}{c}\text { hsa-mir- } \\
4721\end{array}$ \\
\hline TSPYL4 & 40 & $\begin{array}{c}\text { hsa-mir- } \\
4779\end{array}$ & Down & MT1A & 41 & $\begin{array}{c}\text { hsa-mir- } \\
1299\end{array}$ \\
\hline $\begin{array}{c}\text { GABARAPL } \\
1\end{array}$ & 40 & $\begin{array}{l}\text { hsa-mir- } \\
9500\end{array}$ & Down & PYCARD & 40 & $\begin{array}{l}\text { hsa-mir- } \\
6749-3 p\end{array}$ \\
\hline NPTN & 40 & $\begin{array}{l}\text { hsa-mir- } \\
3617-3 p\end{array}$ & Down & EHD4 & 40 & $\begin{array}{l}\text { hsa-mir- } \\
1273 \mathrm{~g}-3 \mathrm{p}\end{array}$ \\
\hline STRBP & 40 & $\begin{array}{l}\text { hsa-mir- } \\
7977\end{array}$ & Down & LYN & 39 & $\begin{array}{c}\text { hsa-mir- } \\
548 v\end{array}$ \\
\hline ATP6V1B2 & 38 & $\begin{array}{c}\text { hsa-mir- } \\
3133\end{array}$ & Down & BAZ2B & 39 & $\begin{array}{c}\text { hsa-mir- } \\
7977\end{array}$ \\
\hline SLC12A5 & 38 & $\begin{array}{l}\text { hsa-mir- } \\
2277-3 p\end{array}$ & Down & FXYD5 & 39 & $\begin{array}{c}\text { hsa-mir- } \\
4322\end{array}$ \\
\hline VPS35 & 37 & $\begin{array}{l}\text { hsa-mir- } \\
6893-5 p\end{array}$ & Down & ITPKB & 38 & $\begin{array}{l}\text { hsa-mir- } \\
\text { 548as-3p }\end{array}$ \\
\hline ARF3 & 36 & $\begin{array}{l}\text { hsa-mir- } \\
6760-5 p\end{array}$ & Down & SLC2A5 & 38 & $\begin{array}{c}\text { hsa-mir- } \\
3650\end{array}$ \\
\hline PNKD & 36 & $\begin{array}{l}\text { hsa-mir- } \\
\text { 380-5p }\end{array}$ & Down & SP110 & 37 & $\begin{array}{l}\text { hsa-mir- } \\
6716-5 p\end{array}$ \\
\hline CDK14 & 35 & $\begin{array}{l}\text { hsa-mir- } \\
5196-5 p\end{array}$ & Down & GIMAP4 & 37 & $\begin{array}{l}\text { hsa-mir- } \\
\text { 548ad-5p }\end{array}$ \\
\hline
\end{tabular}




\begin{tabular}{|c|c|c|c|c|c|c|c|}
\hline $\mathrm{Up}$ & BASP1 & 35 & $\begin{array}{l}\text { hsa-mir- } \\
1255 b-5 p\end{array}$ & Down & TMEM119 & 37 & $\begin{array}{c}\text { hsa-mir- } \\
4698\end{array}$ \\
\hline $\mathrm{Up}$ & CACNB2 & 34 & $\begin{array}{l}\text { hsa-mir- } \\
450 a-2-3 p\end{array}$ & Down & GPC5 & 36 & $\begin{array}{c}\text { hsa-mir- } \\
548 \mathrm{z}\end{array}$ \\
\hline $\mathrm{Up}$ & CYGB & 34 & $\begin{array}{c}\text { hsa-mir- } \\
2392\end{array}$ & Down & MFRP & 36 & $\begin{array}{l}\text { hsa-mir- } \\
6825-5 p\end{array}$ \\
\hline $\mathrm{Up}$ & SLITRK4 & 34 & $\begin{array}{l}\text { hsa-mir- } \\
5580-3 p\end{array}$ & Down & SCIN & 35 & $\begin{array}{c}\text { hsa-mir- } \\
4734\end{array}$ \\
\hline Up & FBXL16 & 34 & $\begin{array}{l}\text { hsa-mir- } \\
1185-1-3 p\end{array}$ & Down & CSRP1 & 34 & $\begin{array}{l}\text { hsa-mir- } \\
7159-3 p\end{array}$ \\
\hline Up & PTPRT & 32 & $\begin{array}{l}\text { hsa-mir- } \\
5006-3 p\end{array}$ & Down & LRIG1 & 34 & $\begin{array}{l}\text { hsa-mir- } \\
3140-3 p\end{array}$ \\
\hline Up & PABPC1L2B & 32 & $\begin{array}{c}\text { hsa-mir- } \\
4306\end{array}$ & Down & EMX2 & 33 & $\begin{array}{l}\text { hsa-mir- } \\
6809-3 p\end{array}$ \\
\hline Up & ETS2 & 31 & $\begin{array}{l}\text { hsa-mir- } \\
\text { 199a-3p }\end{array}$ & Down & NINJ1 & 33 & $\begin{array}{c}\text { hsa-mir- } \\
5095\end{array}$ \\
\hline Up & GABRB3 & 31 & $\begin{array}{l}\text { hsa-mir- } \\
548 \mathrm{j}-3 \mathrm{p}\end{array}$ & Down & SDC4 & 33 & $\begin{array}{l}\text { hsa-mir- } \\
548 b b-3 p\end{array}$ \\
\hline Up & RCAN2 & 31 & $\begin{array}{l}\text { hsa-mir- } \\
513 \mathrm{~b}-3 \mathrm{p}\end{array}$ & Down & SYK & 33 & $\begin{array}{l}\text { hsa-mir- } \\
\text { 203a-3p }\end{array}$ \\
\hline Up & NMNAT2 & 31 & $\begin{array}{l}\text { hsa-mir- } \\
1304-5 p\end{array}$ & Down & WASF2 & 33 & $\begin{array}{l}\text { hsa-mir- } \\
3667-3 p\end{array}$ \\
\hline $\mathrm{Up}$ & RTN4 & 31 & $\begin{array}{l}\text { hsa-mir- } \\
376 c-3 p\end{array}$ & Down & SYTL4 & 33 & $\begin{array}{l}\text { hsa-mir- } \\
\text { 4659b-3p }\end{array}$ \\
\hline $\mathrm{Up}$ & NDRG3 & 31 & $\begin{array}{l}\text { hsa-mir- } \\
2467-3 p\end{array}$ & Down & STAT5A & 32 & $\begin{array}{l}\text { hsa-mir- } \\
7114-3 p\end{array}$ \\
\hline Up & MOAP1 & 31 & $\begin{array}{c}\text { hsa-mir- } \\
4280\end{array}$ & Down & VAMP3 & 32 & $\begin{array}{l}\text { hsa-mir- } \\
1273 \mathrm{~g}-5 \mathrm{p}\end{array}$ \\
\hline Up & PGRMC1 & 30 & $\begin{array}{l}\text { hsa-mir- } \\
6866-5 p\end{array}$ & Down & RASSF2 & 32 & $\begin{array}{c}\text { hsa-mir- } \\
4480\end{array}$ \\
\hline Up & FGF9 & 29 & $\begin{array}{l}\text { hsa-mir- } \\
6817-3 p\end{array}$ & Down & ABCB7 & 30 & $\begin{array}{l}\text { hsa-mir- } \\
\text { 548ai }\end{array}$ \\
\hline
\end{tabular}




\begin{tabular}{|c|c|c|c|c|c|c|c|}
\hline Up & RAP1GAP2 & 28 & $\begin{array}{l}\text { hsa-mir- } \\
7111-5 p\end{array}$ & Down & TPP1 & 30 & $\begin{array}{l}\text { hsa-mir- } \\
\text { 219a-2-3p }\end{array}$ \\
\hline Up & & & & Down & & & hsa-mir- \\
\hline & CELF2 & 27 & $\begin{array}{l}\text { hsa-mir- } \\
\text { 548d-3p }\end{array}$ & & RAB31 & 30 & $\begin{array}{c}1255 b-2- \\
3 p\end{array}$ \\
\hline Up & CREG2 & 27 & $\begin{array}{l}\text { hsa-mir- } \\
4423-5 p\end{array}$ & Down & HAVCR2 & 30 & $\begin{array}{l}\text { hsa-mir- } \\
520 f-3 p\end{array}$ \\
\hline Up & ITPR1 & 26 & $\begin{array}{l}\text { hsa-mir- } \\
449 a\end{array}$ & Down & SIGLEC10 & 30 & $\begin{array}{l}\text { hsa-mir- } \\
6792-3 p\end{array}$ \\
\hline Up & VAMP1 & 26 & $\begin{array}{l}\text { hsa-mir- } \\
3677-3 p\end{array}$ & Down & HLA-DOA & 29 & $\begin{array}{c}\text { hsa-mir- } \\
4329\end{array}$ \\
\hline $\mathrm{Up}$ & AKAP6 & 26 & $\begin{array}{l}\text { hsa-mir- } \\
4701-5 p\end{array}$ & Down & PDPN & 29 & $\begin{array}{l}\text { hsa-mir- } \\
4649-3 p\end{array}$ \\
\hline Up & MICAL2 & 26 & $\begin{array}{c}\text { hsa-mir- } \\
\text { 4436a }\end{array}$ & Down & CNN3 & 28 & $\begin{array}{l}\text { hsa-mir- } \\
4255\end{array}$ \\
\hline Up & RTN3 & 26 & $\begin{array}{l}\text { hsa-mir- } \\
4694-3 p\end{array}$ & Down & DAB2 & 28 & $\begin{array}{l}\text { hsa-mir- } \\
7515\end{array}$ \\
\hline Up & ADCYAP1 & 25 & $\begin{array}{c}\text { hsa-mir- } \\
1284\end{array}$ & Down & MYL12A & 28 & $\begin{array}{l}\text { hsa-mir- } \\
6803-5 p\end{array}$ \\
\hline $\mathrm{Up}$ & IGSF3 & 25 & $\begin{array}{c}\text { hsa-mir- } \\
107\end{array}$ & Down & RHOG & 27 & $\begin{array}{l}\text { hsa-mir- } \\
6728-3 p\end{array}$ \\
\hline Up & VSNL1 & 25 & $\begin{array}{l}\text { hsa-mir- } \\
548 a v-5 p\end{array}$ & Down & LAMP2 & 27 & $\begin{array}{l}\text { hsa-mir- } \\
4537\end{array}$ \\
\hline Up & AGK & 25 & $\begin{array}{c}\text { hsa-mir- } \\
6130\end{array}$ & Down & CEBPD & 26 & $\begin{array}{l}\text { hsa-mir- } \\
\text { 4999-3p }\end{array}$ \\
\hline Up & TMX4 & 25 & $\begin{array}{l}\text { hsa-mir- } \\
4778-3 p\end{array}$ & Down & SIPA1 & 26 & $\begin{array}{l}\text { hsa-mir- } \\
7704\end{array}$ \\
\hline Up & ATP1A1 & 24 & $\begin{array}{l}\text { hsa-mir- } \\
\text { 340-5p }\end{array}$ & Down & VAMP8 & 26 & $\begin{array}{l}\text { hsa-mir- } \\
6832-5 p\end{array}$ \\
\hline $\mathrm{Up}$ & CAMK2G & 24 & $\begin{array}{l}\text { hsa-mir- } \\
4753-3 p\end{array}$ & Down & FAM107A & 26 & $\begin{array}{l}\text { hsa-mir- } \\
6826-3 p\end{array}$ \\
\hline Up & KCNJ12 & 24 & $\begin{array}{l}\text { hsa-mir- } \\
6754-3 p\end{array}$ & Down & LSM11 & 26 & $\begin{array}{l}\text { hsa-mir- } \\
550 a-3-5 p\end{array}$ \\
\hline
\end{tabular}




\begin{tabular}{|c|c|c|c|c|c|c|c|}
\hline $\mathrm{Up}$ & NRGN & 24 & $\begin{array}{c}\text { hsa-mir- } \\
5698\end{array}$ & Down & CEBPB & 25 & $\begin{array}{l}\text { hsa-mir- } \\
548 \mathrm{t}-5 \mathrm{p}\end{array}$ \\
\hline Up & SNAP25 & 24 & $\begin{array}{l}\text { hsa-mir- } \\
\text { 3925-3p }\end{array}$ & Down & RHOQ & 25 & $\begin{array}{c}\text { hsa-mir- } \\
4310\end{array}$ \\
\hline $\mathrm{Up}$ & FRMPD4 & 24 & $\begin{array}{l}\text { hsa-mir- } \\
4279\end{array}$ & Down & CXCL16 & 25 & $\begin{array}{c}\text { hsa-mir- } \\
5694\end{array}$ \\
\hline $\mathrm{Up}$ & STAMBPL1 & 24 & $\begin{array}{l}\text { hsa-mir- } \\
6868-3 p\end{array}$ & Down & RPS27 & 24 & $\begin{array}{c}\text { hsa-mir- } \\
1260 \mathrm{~b}\end{array}$ \\
\hline $\mathrm{Up}$ & SSX2IP & 24 & $\begin{array}{l}\text { hsa-mir- } \\
5189-3 p\end{array}$ & Down & ATP6V0E1 & 24 & $\begin{array}{c}\text { hsa-mir- } \\
5739\end{array}$ \\
\hline $\mathrm{Up}$ & GABRG2 & 23 & $\begin{array}{c}\text { hsa-mir- } \\
1470\end{array}$ & Down & PALLD & 24 & $\begin{array}{l}\text { hsa-mir- } \\
1250-3 p\end{array}$ \\
\hline $\mathrm{Up}$ & CD200 & 23 & $\begin{array}{c}\text { hsa-mir- } \\
1260 b\end{array}$ & Down & HOXB6 & 23 & $\begin{array}{c}\text { hsa-mir- } \\
4688\end{array}$ \\
\hline Up & PCSK1 & 23 & $\begin{array}{l}\text { hsa-mir- } \\
1185-2-3 p\end{array}$ & Down & EZR & 23 & $\begin{array}{c}\text { hsa-mir- } \\
3660\end{array}$ \\
\hline Up & AP3B2 & 23 & $\begin{array}{l}\text { hsa-mir- } \\
4781-3 p\end{array}$ & Down & FERMT2 & 23 & $\begin{array}{c}\text { hsa-mir- } \\
4299\end{array}$ \\
\hline $\mathrm{Up}$ & ACP1 & 22 & $\begin{array}{c}\text { hsa-mir- } \\
5680\end{array}$ & Down & VASN & 23 & $\begin{array}{l}\text { hsa-mir- } \\
4690-5 p\end{array}$ \\
\hline $\mathrm{Up}$ & ELAVL4 & 22 & $\begin{array}{c}\text { hsa-mir- } \\
4635\end{array}$ & Down & SERPINA3 & 22 & $\begin{array}{l}\text { hsa-mir- } \\
4776-3 p\end{array}$ \\
\hline $\mathrm{Up}$ & PPP1R2 & 22 & $\begin{array}{l}\text { hsa-mir- } \\
\text { 190a-3p }\end{array}$ & Down & LAT2 & 22 & $\begin{array}{l}\text { hsa-mir- } \\
4667-5 p\end{array}$ \\
\hline $\mathrm{Up}$ & TPM1 & 22 & $\begin{array}{c}\text { hsa-mir- } \\
6071\end{array}$ & Down & HAP1 & 22 & $\begin{array}{l}\text { hsa-mir- } \\
6797-5 p\end{array}$ \\
\hline $\mathrm{Up}$ & NRXN1 & 22 & $\begin{array}{l}\text { hsa-mir- } \\
1537-5 p\end{array}$ & Down & $\mathrm{DIO} 2$ & 21 & $\begin{array}{c}\text { hsa-mir- } \\
4453\end{array}$ \\
\hline $\mathrm{Up}$ & DIRAS2 & 22 & $\begin{array}{l}\text { hsa-mir- } \\
5010-5 p\end{array}$ & Down & ARHGEF6 & 21 & $\begin{array}{l}\text { hsa-mir- } \\
5197-3 p\end{array}$ \\
\hline Up & OXR1 & 22 & $\begin{array}{c}\text { hsa-mir- } \\
586\end{array}$ & Down & LHPP & 21 & $\begin{array}{l}\text { hsa-mir- } \\
4763-3 p\end{array}$ \\
\hline
\end{tabular}




\begin{tabular}{|c|c|c|c|c|c|c|c|}
\hline $\mathrm{Up}$ & NECAP1 & 21 & $\begin{array}{c}\text { hsa-mir- } \\
6132\end{array}$ & Down & ANTXR1 & 21 & $\begin{array}{c}\text { hsa-mir- } \\
4469\end{array}$ \\
\hline Up & RAB6B & 21 & $\begin{array}{c}\text { hsa-mir- } \\
8085\end{array}$ & Down & EMID1 & 21 & $\begin{array}{l}\text { hsa-mir- } \\
6777-5 p\end{array}$ \\
\hline $\mathrm{Up}$ & CYP26B1 & 21 & $\begin{array}{c}\text { hsa-mir- } \\
5697\end{array}$ & Down & CD86 & 20 & $\begin{array}{c}\text { hsa-mir- } \\
8055\end{array}$ \\
\hline Up & NECAB1 & 21 & $\begin{array}{l}\text { hsa-mir- } \\
\text { 3074-3p }\end{array}$ & Down & GSTM3 & 20 & $\begin{array}{l}\text { hsa-mir- } \\
6778-3 p\end{array}$ \\
\hline Up & NELL2 & 20 & $\begin{array}{l}\text { hsa-mir- } \\
6810-3 p\end{array}$ & Down & UCP2 & 20 & $\begin{array}{c}\text { hsa-mir- } \\
1321\end{array}$ \\
\hline Up & PTPRN2 & 20 & $\begin{array}{l}\text { hsa-mir- } \\
371 b-5 p\end{array}$ & Down & KANK1 & 20 & $\begin{array}{l}\text { hsa-mir- } \\
3622 b-5 p\end{array}$ \\
\hline $\mathrm{Up}$ & SLC30A3 & 20 & $\begin{array}{l}\text { hsa-mir- } \\
7846-3 p\end{array}$ & Down & HSPB8 & 20 & $\begin{array}{c}\text { hsa-mir- } \\
4491\end{array}$ \\
\hline Up & EPB41L3 & 20 & $\begin{array}{l}\text { hsa-mir- } \\
7161-5 p\end{array}$ & Down & SEC61A1 & 20 & $\begin{array}{c}\text { hsa-mir- } \\
1253\end{array}$ \\
\hline Up & PPP3CB & 19 & $\begin{array}{l}\text { hsa-mir- } \\
\text { 181a-2-3p }\end{array}$ & Down & OAS2 & 19 & $\begin{array}{c}\text { hsa-mir- } \\
4635\end{array}$ \\
\hline Up & MAPK9 & 19 & $\begin{array}{l}\text { hsa-mir- } \\
1178-3 p\end{array}$ & Down & SLC11A1 & 19 & $\begin{array}{c}\text { hsa-mir- } \\
4419 \mathrm{a}\end{array}$ \\
\hline $\mathrm{Up}$ & LDOC1 & 19 & $\begin{array}{c}\text { hsa-mir- } \\
8073\end{array}$ & Down & FGFRL1 & 19 & $\begin{array}{l}\text { hsa-mir- } \\
\text { 3130-3p }\end{array}$ \\
\hline Up & EPDR1 & 19 & $\begin{array}{l}\text { hsa-mir- } \\
5692 \mathrm{~b}\end{array}$ & Down & GPT2 & 19 & $\begin{array}{c}\text { hsa-mir- } \\
5093\end{array}$ \\
\hline Up & ZNF25 & 19 & $\begin{array}{c}\text { hsa-mir- } \\
3660\end{array}$ & Down & GLIS3 & 19 & $\begin{array}{l}\text { hsa-mir- } \\
138-2-3 p\end{array}$ \\
\hline Up & PFN2 & 18 & $\begin{array}{l}\text { hsa-mir- } \\
3200-5 p\end{array}$ & Down & ADD3 & 18 & $\begin{array}{c}\text { hsa-mir- } \\
5692 \mathrm{c}\end{array}$ \\
\hline Up & GAS7 & 18 & $\begin{array}{l}\text { hsa-mir- } \\
\text { 548aw }\end{array}$ & Down & AXL & 18 & $\begin{array}{l}\text { hsa-mir- } \\
5699-3 p\end{array}$ \\
\hline Up & CLSTN3 & 17 & $\begin{array}{l}\text { hsa-mir- } \\
4789-3 p\end{array}$ & Down & CD68 & 18 & $\begin{array}{c}\text { hsa-mir- } \\
4469\end{array}$ \\
\hline
\end{tabular}




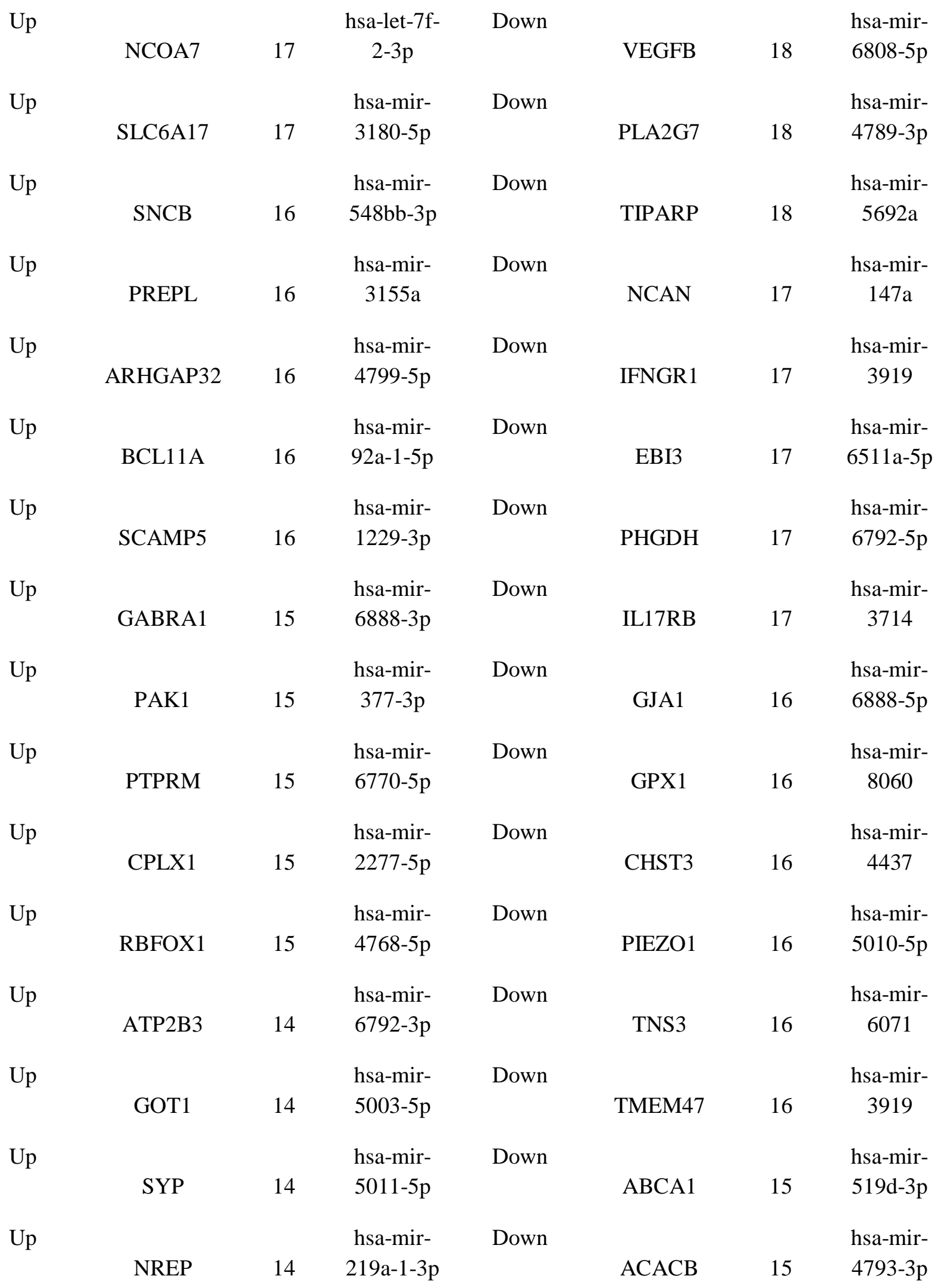




\begin{tabular}{|c|c|c|c|c|c|c|c|}
\hline $\mathrm{Up}$ & CAMTA1 & 14 & $\begin{array}{l}\text { hsa-mir- } \\
\text { 20a-5p }\end{array}$ & Down & CNGB1 & 15 & $\begin{array}{l}\text { hsa-mir- } \\
4680-5 p\end{array}$ \\
\hline Up & CNTNAP2 & 14 & $\begin{array}{c}\text { hsa-mir- } \\
4444\end{array}$ & Down & SPTBN1 & 15 & $\begin{array}{l}\text { hsa-mir- } \\
1301-3 p\end{array}$ \\
\hline $\mathrm{Up}$ & FAM216A & 14 & $\begin{array}{l}\text { hsa-mir- } \\
4735-5 p\end{array}$ & Down & GPR37L1 & 15 & $\begin{array}{l}\text { hsa-mir- } \\
642 a-5 p\end{array}$ \\
\hline Up & SMYD2 & 14 & $\begin{array}{l}\text { hsa-mir- } \\
5588-3 \text { p }\end{array}$ & Down & EXOG & 15 & $\begin{array}{l}\text { hsa-mir- } \\
642 b-3 p\end{array}$ \\
\hline Up & GNG3 & 13 & $\begin{array}{l}\text { hsa-mir- } \\
7106-5 p\end{array}$ & Down & ARRDC2 & 15 & $\begin{array}{l}\text { hsa-mir- } \\
411-3 p\end{array}$ \\
\hline Up & IARS & 13 & $\begin{array}{c}\text { hsa-mir- } \\
100-5 \mathrm{p}\end{array}$ & Down & SMOX & 15 & $\begin{array}{c}\text { hsa-mir- } \\
\text { 3689d }\end{array}$ \\
\hline $\mathrm{Up}$ & MAP4 & 13 & $\begin{array}{l}\text { hsa-mir- } \\
551 \mathrm{~b}-3 \mathrm{p}\end{array}$ & Down & BCAN & 15 & $\begin{array}{l}\text { hsa-mir- } \\
6780 \mathrm{~b}-3 \mathrm{p}\end{array}$ \\
\hline Up & NSF & 13 & $\begin{array}{l}\text { hsa-mir- } \\
103 a-3 p\end{array}$ & Down & FAM107B & 15 & $\begin{array}{c}\text { hsa-mir- } \\
759\end{array}$ \\
\hline Up & PGAM1 & 13 & $\begin{array}{c}\text { hsa-mir- } \\
589-5 p\end{array}$ & Down & GNA12 & 14 & $\begin{array}{c}\text { hsa-mir- } \\
4309\end{array}$ \\
\hline Up & MLLT11 & 13 & $\begin{array}{c}\text { hsa-mir- } \\
3202\end{array}$ & Down & HLA-DRA & 14 & $\begin{array}{l}\text { hsa-mir- } \\
\text { 3928-3p }\end{array}$ \\
\hline Up & CERS6 & 13 & $\begin{array}{l}\text { hsa-mir- } \\
5088-3 \text { p }\end{array}$ & Down & PLEK & 14 & $\begin{array}{c}\text { hsa-mir- } \\
579-3 \text { p }\end{array}$ \\
\hline Up & VGF & 12 & $\begin{array}{l}\text { hsa-mir- } \\
1908-5 p\end{array}$ & Down & LILRB1 & 14 & $\begin{array}{l}\text { hsa-mir- } \\
4789-3 p\end{array}$ \\
\hline Up & KALRN & 12 & $\begin{array}{l}\text { hsa-mir- } \\
5000-5 p\end{array}$ & Down & GPRC5B & 14 & $\begin{array}{c}\text { hsa-mir- } \\
548 \mathrm{~s}\end{array}$ \\
\hline Up & SLC9A6 & 12 & $\begin{array}{c}\text { hsa-mir- } \\
19 a-3 p\end{array}$ & Down & ALDH7A1 & 13 & $\begin{array}{l}\text { hsa-mir- } \\
5571-5 p\end{array}$ \\
\hline Up & PNMA3 & 12 & $\begin{array}{l}\text { hsa-mir- } \\
22-5 p\end{array}$ & Down & PRCP & 13 & $\begin{array}{c}\text { hsa-mir- } \\
4669\end{array}$ \\
\hline Up & SCN3B & 12 & $\begin{array}{l}\text { hsa-mir- } \\
1910-3 p\end{array}$ & Down & TBL1X & 13 & $\begin{array}{c}\text { hsa-mir- } \\
4330\end{array}$ \\
\hline
\end{tabular}




\begin{tabular}{|c|c|c|c|c|c|c|c|}
\hline Up & BEX4 & 12 & $\begin{array}{c}\text { hsa-mir- } \\
3941\end{array}$ & Down & TLR5 & 13 & $\begin{array}{l}\text { hsa-mir- } \\
6780 b-5 p\end{array}$ \\
\hline $\mathrm{Up}$ & PITHD1 & 12 & $\begin{array}{c}\text { hsa-mir- } \\
4511\end{array}$ & Down & HSPA1A & 12 & $\begin{array}{c}\text { hsa-mir- } \\
4424\end{array}$ \\
\hline Up & PDE1A & 11 & $\begin{array}{l}\text { hsa-mir- } \\
5587-5 p\end{array}$ & Down & SLC7A7 & 12 & $\begin{array}{c}\text { hsa-mir- } \\
\text { 3689f }\end{array}$ \\
\hline Up & SCN1B & 11 & $\begin{array}{l}\text { hsa-mir- } \\
4755-3 p\end{array}$ & Down & ADAP2 & 12 & $\begin{array}{c}\text { hsa-mir- } \\
3199\end{array}$ \\
\hline Up & PARM1 & 11 & $\begin{array}{c}\text { hsa-mir- } \\
4422\end{array}$ & Down & PARVG & 12 & $\begin{array}{l}\text { hsa-mir- } \\
4802-5 p\end{array}$ \\
\hline Up & ADARB1 & 10 & $\begin{array}{l}\text { hsa-mir- } \\
218-5 p\end{array}$ & Down & SFXN5 & 12 & $\begin{array}{c}\text { hsa-mir- } \\
4801\end{array}$ \\
\hline Up & ENC1 & 10 & $\begin{array}{l}\text { hsa-mir- } \\
505-5 \mathrm{p}\end{array}$ & Down & BGN & 11 & $\begin{array}{c}\text { hsa-mir- } \\
8485\end{array}$ \\
\hline Up & ELMO1 & 10 & $\begin{array}{c}\text { hsa-mir- } \\
1323\end{array}$ & Down & STOM & 11 & $\begin{array}{l}\text { hsa-mir- } \\
7162-5 p\end{array}$ \\
\hline Up & MAL2 & 10 & $\begin{array}{l}\text { hsa-mir- } \\
6715 a-3 p\end{array}$ & Down & MT1E & 11 & $\begin{array}{l}\text { hsa-mir- } \\
4668-3 p\end{array}$ \\
\hline Up & TMEM130 & 10 & $\begin{array}{c}\text { hsa-mir- } \\
3650\end{array}$ & Down & MT2A & 11 & $\begin{array}{c}\text { hsa-let-7g- } \\
3 \mathrm{p}\end{array}$ \\
\hline Up & OPCML & 9 & $\begin{array}{c}\text { hsa-mir- } \\
3149\end{array}$ & Down & HIPK2 & 11 & $\begin{array}{l}\text { hsa-mir- } \\
760\end{array}$ \\
\hline Up & GNG2 & 9 & $\begin{array}{l}\text { hsa-mir- } \\
16-1-3 p\end{array}$ & Down & MOB3C & 11 & $\begin{array}{l}\text { hsa-mir- } \\
4707-5 p\end{array}$ \\
\hline Up & PDXP & 9 & $\begin{array}{l}\text { hsa-mir- } \\
4662 a-5 p\end{array}$ & Down & ALOX5AP & 10 & $\begin{array}{c}\text { hsa-mir- } \\
4775\end{array}$ \\
\hline Up & KCNJ4 & 8 & $\begin{array}{l}\text { hsa-mir- } \\
3667-3 p\end{array}$ & Down & HDAC1 & 10 & $\begin{array}{l}\text { hsa-mir- } \\
520 \mathrm{~h}\end{array}$ \\
\hline Up & PRKCE & 8 & $\begin{array}{l}\text { hsa-mir- } \\
205-5 p\end{array}$ & Down & MOBP & 10 & $\begin{array}{c}\text { hsa-mir- } \\
3941\end{array}$ \\
\hline Up & BHLHE22 & 8 & $\begin{array}{l}\text { hsa-mir- } \\
4733-5 p\end{array}$ & Down & ATP8B4 & 10 & $\begin{array}{l}\text { hsa-mir- } \\
\text { 208b-5p }\end{array}$ \\
\hline
\end{tabular}




\begin{tabular}{|c|c|c|c|c|c|c|}
\hline REEP1 & 8 & $\begin{array}{l}\text { hsa-mir- } \\
\text { 3680-3p }\end{array}$ & Down & PAQR8 & 10 & $\begin{array}{c}\text { hsa-mir- } \\
557\end{array}$ \\
\hline KRT222 & 8 & $\begin{array}{l}\text { hsa-mir- } \\
654-3 \mathrm{p}\end{array}$ & Down & BCL6 & 9 & $\begin{array}{c}\text { hsa-mir- } \\
339-5 p\end{array}$ \\
\hline FAM19A1 & 8 & $\begin{array}{c}\text { hsa-mir- } \\
607\end{array}$ & Down & FGFR3 & 9 & $\begin{array}{c}\text { hsa-mir- } \\
100-5 p\end{array}$ \\
\hline ASNS & 7 & $\begin{array}{c}\text { hsa-mir- } \\
17-5 p\end{array}$ & Down & LAPTM5 & 9 & $\begin{array}{c}\text { hsa-mir- } \\
1273 \mathrm{e}\end{array}$ \\
\hline COL5A2 & 7 & $\begin{array}{l}\text { hsa-mir- } \\
\text { 29b-3p }\end{array}$ & Down & BCAS1 & 9 & $\begin{array}{c}\text { hsa-mir- } \\
933\end{array}$ \\
\hline DPP6 & 7 & $\begin{array}{l}\text { hsa-mir- } \\
6733-5 p\end{array}$ & Down & DHRS3 & 9 & $\begin{array}{l}\text { hsa-mir- } \\
130 b-5 p\end{array}$ \\
\hline EGR1 & 7 & $\begin{array}{l}\text { hsa-mir- } \\
\text { 377-3p }\end{array}$ & Down & TSC22D4 & 9 & $\begin{array}{l}\text { hsa-mir- } \\
5699-5 p\end{array}$ \\
\hline WASF1 & 7 & $\begin{array}{c}\text { hsa-mir- } \\
411-5 p\end{array}$ & Down & OAF & 9 & $\begin{array}{c}\text { hsa-mir- } \\
1248\end{array}$ \\
\hline TUBB3 & 7 & $\begin{array}{l}\text { hsa-mir- } \\
\text { 200c-3p }\end{array}$ & Down & LCP1 & 8 & $\begin{array}{l}\text { hsa-mir- } \\
520 a-3 p\end{array}$ \\
\hline TBR1 & 7 & $\begin{array}{l}\text { hsa-mir- } \\
7151-5 p\end{array}$ & Down & DCLRE1C & 8 & $\begin{array}{c}\text { hsa-mir- } \\
548 \mathrm{n}\end{array}$ \\
\hline CAMK1D & 7 & $\begin{array}{c}\text { hsa-mir- } \\
5697\end{array}$ & Down & CEBPA & 7 & $\begin{array}{c}\text { hsa-mir- } \\
744-5 p\end{array}$ \\
\hline RASL11B & 7 & $\begin{array}{l}\text { hsa-mir- } \\
4743-3 p\end{array}$ & Down & CLIC1 & 7 & $\begin{array}{c}\text { hsa-mir- } \\
106 b-3 p\end{array}$ \\
\hline NCALD & 7 & $\begin{array}{c}\text { hsa-mir- } \\
\text { 32-3p }\end{array}$ & Down & INPPL1 & 7 & $\begin{array}{c}\text { hsa-mir- } \\
671-5 p\end{array}$ \\
\hline ATP6V1E1 & 6 & $\begin{array}{l}\text { hsa-mir- } \\
221-3 p\end{array}$ & Down & PLOD1 & 7 & $\begin{array}{c}\text { hsa-mir- } \\
23 b-3 p\end{array}$ \\
\hline GPM6A & 6 & $\begin{array}{l}\text { hsa-mir- } \\
\text { 548e-5p }\end{array}$ & Down & RRBP1 & 7 & $\begin{array}{c}\text { hsa-mir- } \\
331-3 p\end{array}$ \\
\hline XK & 6 & $\begin{array}{c}\text { hsa-mir- } \\
31-3 p\end{array}$ & Down & LEMD3 & 7 & $\begin{array}{c}\text { hsa-mir- } \\
10 \mathrm{~b}-5 \mathrm{p}\end{array}$ \\
\hline
\end{tabular}




\begin{tabular}{|c|c|c|c|c|c|c|c|}
\hline Up & GABBR2 & 6 & $\begin{array}{l}\text { hsa-mir- } \\
450 \mathrm{~b}-5 \mathrm{p}\end{array}$ & Down & TMEM51 & 7 & $\begin{array}{c}\text { hsa-mir- } \\
4672\end{array}$ \\
\hline $\mathrm{Up}$ & CBLN2 & 6 & $\begin{array}{l}\text { hsa-mir- } \\
4753-3 p\end{array}$ & Down & ASAP3 & 7 & $\begin{array}{l}\text { hsa-mir- } \\
499 a-5 p\end{array}$ \\
\hline Up & CKMT1A & 6 & $\begin{array}{l}\text { hsa-mir- } \\
451 b\end{array}$ & Down & CGNL1 & 7 & $\begin{array}{l}\text { hsa-mir- } \\
125 a-5 p\end{array}$ \\
\hline Up & OPA1 & 5 & $\begin{array}{l}\text { hsa-mir- } \\
\text { 320a }\end{array}$ & Down & CD151 & 6 & $\begin{array}{l}\text { hsa-mir- } \\
22-3 p\end{array}$ \\
\hline Up & RGS4 & 5 & $\begin{array}{l}\text { hsa-mir- } \\
6867-5 p\end{array}$ & Down & CTSC & 6 & $\begin{array}{l}\text { hsa-mir- } \\
\text { 199a-5p }\end{array}$ \\
\hline Up & UCHL1 & 5 & $\begin{array}{l}\text { hsa-mir- } \\
181 a-5 p\end{array}$ & Down & GBP2 & 6 & $\begin{array}{l}\text { hsa-mir- } \\
4709-5 p\end{array}$ \\
\hline Up & HERC1 & 5 & $\begin{array}{l}\text { hsa-mir- } \\
455-3 p\end{array}$ & Down & ITGB4 & 6 & $\begin{array}{c}\text { hsa-mir- } \\
16-5 \mathrm{p}\end{array}$ \\
\hline Up & INA & 5 & $\begin{array}{l}\text { hsa-mir- } \\
101-3 p\end{array}$ & Down & HTRA1 & 6 & $\begin{array}{l}\text { hsa-mir- } \\
635\end{array}$ \\
\hline Up & PNMA1 & 5 & $\begin{array}{l}\text { hsa-mir- } \\
\text { 30a-5p }\end{array}$ & Down & STK3 & 6 & $\begin{array}{c}\text { hsa-mir- } \\
133 b\end{array}$ \\
\hline Up & CPLX2 & 5 & $\begin{array}{l}\text { hsa-mir- } \\
6765-3 p\end{array}$ & Down & RAPGEF3 & 6 & $\begin{array}{c}\text { hsa-mir- } \\
4286\end{array}$ \\
\hline Up & TMEM14A & 5 & $\begin{array}{l}\text { hsa-mir- } \\
\text { 148a-3p }\end{array}$ & Down & RAI14 & 6 & $\begin{array}{l}\text { hsa-mir- } \\
122-5 p\end{array}$ \\
\hline Up & AFTPH & 5 & $\begin{array}{l}\text { hsa-mir- } \\
\text { 374b-5p }\end{array}$ & Down & FOXO4 & 5 & $\begin{array}{c}\text { hsa-mir- } \\
1274 \mathrm{a}\end{array}$ \\
\hline Up & ACTR 10 & 5 & $\begin{array}{l}\text { hsa-mir- } \\
4668-5 p\end{array}$ & Down & NTRK2 & 5 & $\begin{array}{l}\text { hsa-mir- } \\
\text { 200c-3p }\end{array}$ \\
\hline Up & CMAS & 5 & $\begin{array}{l}\text { hsa-mir- } \\
\text { 320b }\end{array}$ & Down & HEPH & 5 & $\begin{array}{l}\text { hsa-mir- } \\
4786-3 p\end{array}$ \\
\hline Up & DDX24 & 5 & $\begin{array}{l}\text { hsa-mir- } \\
769-5 p\end{array}$ & Down & FARP1 & 5 & $\begin{array}{c}\text { hsa-let-7f- } \\
5 \mathrm{p}\end{array}$ \\
\hline Up & SYT13 & 5 & $\begin{array}{c}\text { hsa-mir- } \\
4688\end{array}$ & Down & NEK6 & 5 & $\begin{array}{l}\text { hsa-mir- } \\
1307-3 p\end{array}$ \\
\hline
\end{tabular}




\begin{tabular}{|c|c|c|c|c|c|c|c|}
\hline Up & ADCY1 & 4 & $\begin{array}{c}\text { hsa-mir- } \\
331-3 p\end{array}$ & Down & SASH3 & 5 & $\begin{array}{l}\text { hsa-mir- } \\
374 c-3 p\end{array}$ \\
\hline Up & DOCK3 & 4 & $\begin{array}{c}\text { hsa-mir- } \\
186-5 \mathrm{p}\end{array}$ & Down & RIN2 & 5 & $\begin{array}{l}\text { hsa-mir- } \\
\text { 200b-3p }\end{array}$ \\
\hline $\mathrm{Up}$ & FGF12 & 4 & $\begin{array}{c}\text { hsa-mir- } \\
590-5 p\end{array}$ & Down & PLEKHB1 & 5 & $\begin{array}{c}\text { hsa-mir-7 } \\
5 \mathrm{p}\end{array}$ \\
\hline Up & OAT & 4 & $\begin{array}{c}\text { hsa-mir- } \\
99 \mathrm{~b}-5 \mathrm{p}\end{array}$ & Down & ZNF462 & 5 & $\begin{array}{c}\text { hsa-mir- } \\
320 \mathrm{a}\end{array}$ \\
\hline Up & STXBP1 & 4 & $\begin{array}{c}\text { hsa-mir- } \\
544 a\end{array}$ & Down & AHNAK & 5 & $\begin{array}{c}\text { hsa-mir- } \\
421\end{array}$ \\
\hline Up & SYT1 & 4 & $\begin{array}{c}\text { hsa-mir- } \\
8064\end{array}$ & Down & FAM181B & 5 & $\begin{array}{l}\text { hsa-mir- } \\
4753-3 p\end{array}$ \\
\hline Up & TAC1 & 4 & $\begin{array}{c}\text { hsa-mir- } \\
206\end{array}$ & Down & DDR1 & 4 & $\begin{array}{c}\text { hsa-mir- } \\
199 a-5 p\end{array}$ \\
\hline Up & TUSC3 & 4 & $\begin{array}{c}\text { hsa-mir- } \\
25-3 p\end{array}$ & Down & HLA-DQA1 & 4 & $\begin{array}{l}\text { hsa-mir- } \\
6798-3 p\end{array}$ \\
\hline Up & CCNA1 & 4 & $\begin{array}{c}\text { hsa-mir- } \\
372-3 p\end{array}$ & Down & PDK4 & 4 & $\begin{array}{l}\text { hsa-mir- } \\
103 a-3 p\end{array}$ \\
\hline Up & LDB2 & 4 & $\begin{array}{c}\text { hsa-mir- } \\
3169\end{array}$ & Down & S100A1 & 4 & $\begin{array}{c}\text { hsa-mir- } \\
138-5 p\end{array}$ \\
\hline Up & MAGED1 & 4 & $\begin{array}{c}\text { hsa-mir- } \\
760\end{array}$ & Down & TIMP1 & 4 & $\begin{array}{l}\text { hsa-mir- } \\
\text { 519a-3p }\end{array}$ \\
\hline Up & ACOT7 & 4 & $\begin{array}{l}\text { hsa-mir- } \\
1229-3 p\end{array}$ & Down & TP53BP2 & 4 & $\begin{array}{l}\text { hsa-mir- } \\
\text { 222-3p }\end{array}$ \\
\hline Up & DMXL2 & 4 & $\begin{array}{c}\text { hsa-mir- } \\
19 b-3 p\end{array}$ & Down & BBOX1 & 4 & $\begin{array}{c}\text { hsa-mir- } \\
4477 \mathrm{a}\end{array}$ \\
\hline Up & SYBU & 4 & $\begin{array}{c}\text { hsa-mir- } \\
93-5 p\end{array}$ & Down & SELENBP1 & 4 & $\begin{array}{c}\text { hsa-mir- } \\
20 a-5 p\end{array}$ \\
\hline Up & LINGO1 & 4 & $\begin{array}{c}\text { hsa-mir- } \\
6077\end{array}$ & Down & SLC9A3R1 & 4 & $\begin{array}{c}\text { hsa-mir- } \\
877-3 p\end{array}$ \\
\hline Up & CADPS2 & 4 & $\begin{array}{l}\text { hsa-mir- } \\
\text { 30c-5p }\end{array}$ & Down & IFITM3 & 4 & $\begin{array}{c}\text { hsa-mir- } \\
3674\end{array}$ \\
\hline
\end{tabular}




\begin{tabular}{|c|c|c|c|c|c|c|c|}
\hline Up & CCKBR & 3 & $\begin{array}{l}\text { hsa-mir- } \\
148 b-3 p\end{array}$ & Down & ANGPTL4 & 4 & $\begin{array}{c}\text { hsa-mir-1- } \\
1\end{array}$ \\
\hline $\mathrm{Up}$ & ENO2 & 3 & $\begin{array}{l}\text { hsa-mir- } \\
\text { 382-3p }\end{array}$ & Down & RHBDF2 & 4 & $\begin{array}{c}\text { hsa-mir- } \\
98-5 p\end{array}$ \\
\hline Up & MDH1 & 3 & $\begin{array}{c}\text { hsa-mir- } \\
15 \mathrm{~b}-5 \mathrm{p}\end{array}$ & Down & UNC93B1 & 4 & $\begin{array}{c}\text { hsa-mir-1- } \\
3 \mathrm{p}\end{array}$ \\
\hline Up & $\mathrm{NEFH}$ & 3 & $\begin{array}{l}\text { hsa-mir- } \\
106 b-5 p\end{array}$ & Down & ACSS1 & 4 & $\begin{array}{c}\text { hsa-mir- } \\
191-5 p\end{array}$ \\
\hline Up & PI4KA & 3 & $\begin{array}{c}\text { hsa-mir- } \\
484\end{array}$ & Down & СMTM3 & 4 & $\begin{array}{c}\text { hsa-mir- } \\
132-3 p\end{array}$ \\
\hline $\mathrm{Up}$ & STX1A & 3 & $\begin{array}{l}\text { hsa-mir- } \\
34 a-5 p\end{array}$ & Down & APOE & 3 & $\begin{array}{l}\text { hsa-mir- } \\
1908-5 p\end{array}$ \\
\hline Up & VAMP2 & 3 & $\begin{array}{c}\text { hsa-mir- } \\
206\end{array}$ & Down & CSF1R & 3 & $\begin{array}{c}\text { hsa-mir- } \\
34 a-5 p\end{array}$ \\
\hline Up & ZNF365 & 3 & $\begin{array}{l}\text { hsa-mir-7- } \\
5 \mathrm{p}\end{array}$ & Down & CSK & 3 & $\begin{array}{l}\text { hsa-mir- } \\
1180-3 p\end{array}$ \\
\hline Up & CYFIP2 & 3 & $\begin{array}{l}\text { hsa-mir-9- } \\
5 \mathrm{p}\end{array}$ & Down & EMP3 & 3 & $\begin{array}{c}\text { hsa-mir-1- } \\
1\end{array}$ \\
\hline Up & FBXO34 & 3 & $\begin{array}{l}\text { hsa-mir- } \\
181 a-5 p\end{array}$ & Down & ITGB2 & 3 & $\begin{array}{l}\text { hsa-mir- } \\
146 a-5 p\end{array}$ \\
\hline Up & SLC6A15 & 3 & $\begin{array}{l}\text { hsa-mir- } \\
23 a-3 p\end{array}$ & Down & ITGB5 & 3 & $\begin{array}{c}\text { hsa-mir- } \\
155-5 \mathrm{p}\end{array}$ \\
\hline $\mathrm{Up}$ & BEX1 & 3 & $\begin{array}{c}\text { hsa-mir- } \\
124-3 p\end{array}$ & Down & LRP4 & 3 & $\begin{array}{c}\text { hsa-mir- } \\
197-3 p\end{array}$ \\
\hline $\mathrm{Up}$ & TSPYL2 & 3 & $\begin{array}{c}\text { hsa-mir- } \\
15 a-5 p\end{array}$ & Down & SPP1 & 3 & $\begin{array}{l}\text { hsa-mir- } \\
299-5 p\end{array}$ \\
\hline Up & CPNE4 & 3 & $\begin{array}{l}\text { hsa-mir- } \\
148 b-3 p\end{array}$ & Down & TGFB3 & 3 & $\begin{array}{c}\text { hsa-mir- } \\
29 a-3 p\end{array}$ \\
\hline Up & STEAP2 & 3 & $\begin{array}{c}\text { hsa-mir- } \\
940\end{array}$ & Down & TST & 3 & $\begin{array}{c}\text { hsa-let-7d- } \\
5 \mathrm{p}\end{array}$ \\
\hline Up & ART3 & 2 & $\begin{array}{c}\text { hsa-mir- } \\
122-5 p\end{array}$ & Down & TEAD2 & 3 & $\begin{array}{l}\text { hsa-mir- } \\
346\end{array}$ \\
\hline
\end{tabular}




\begin{tabular}{|c|c|c|c|c|c|c|c|}
\hline Up & ATP1B1 & 2 & $\begin{array}{l}\text { hsa-mir- } \\
218-5 p\end{array}$ & Down & PLOD3 & 3 & $\begin{array}{l}\text { hsa-mir- } \\
21-5 p\end{array}$ \\
\hline Up & CHN1 & 2 & $\begin{array}{l}\text { hsa-mir- } \\
1301-3 p\end{array}$ & Down & MERTK & 3 & $\begin{array}{c}\text { hsa-mir- } \\
126-3 p\end{array}$ \\
\hline Up & CIRBP & 2 & $\begin{array}{c}\text { hsa-mir- } \\
\text { 30a-5p }\end{array}$ & Down & SLCO2B1 & 3 & $\begin{array}{l}\text { hsa-mir- } \\
640\end{array}$ \\
\hline Up & СКМТ1B & 2 & $\begin{array}{l}\text { hsa-mir- } \\
\text { 92a-3p }\end{array}$ & Down & CLEC5A & 3 & $\begin{array}{l}\text { hsa-mir- } \\
125 a-5 p\end{array}$ \\
\hline $\mathrm{Up}$ & DLX1 & 2 & $\begin{array}{l}\text { hsa-mir- } \\
193 b-3 p\end{array}$ & Down & NUPR1 & 3 & $\begin{array}{c}\text { hsa-mir- } \\
615-3 p\end{array}$ \\
\hline Up & EXTL2 & 2 & $\begin{array}{l}\text { hsa-mir- } \\
215-5 p\end{array}$ & Down & HERC5 & 3 & $\begin{array}{l}\text { hsa-mir- } \\
3529-3 p\end{array}$ \\
\hline Up & GLS & 2 & $\begin{array}{c}\text { hsa-mir-7- } \\
5 \mathrm{p}\end{array}$ & Down & S100A16 & 3 & $\begin{array}{l}\text { hsa-mir- } \\
193 b-3 p\end{array}$ \\
\hline $\mathrm{Up}$ & KLC1 & 2 & $\begin{array}{c}\text { hsa-mir- } \\
197-3 p\end{array}$ & Down & APLNR & 2 & $\begin{array}{l}\text { hsa-mir- } \\
\text { 10b-5p }\end{array}$ \\
\hline $\mathrm{Up}$ & MAP2 & 2 & $\begin{array}{c}\text { hsa-mir- } \\
382-3 p\end{array}$ & Down & PTTG1IP & 2 & $\begin{array}{c}\text { hsa-mir- } \\
124-3 p\end{array}$ \\
\hline Up & NEFM & 2 & $\begin{array}{l}\text { hsa-let-7a- } \\
5 \mathrm{p}\end{array}$ & Down & CYBA & 2 & $\begin{array}{c}\text { hsa-mir- } \\
15 \mathrm{~b}-5 \mathrm{p}\end{array}$ \\
\hline $\mathrm{Up}$ & NNAT & 2 & $\begin{array}{l}\text { hsa-mir- } \\
708-5 p\end{array}$ & Down & LPAR1 & 2 & $\begin{array}{l}\text { hsa-mir- } \\
200 c-3 p\end{array}$ \\
\hline Up & SH3GL2 & 2 & $\begin{array}{l}\text { hsa-mir- } \\
\text { 330-3p }\end{array}$ & Down & EPHX1 & 2 & $\begin{array}{l}\text { hsa-mir- } \\
193 b-3 p\end{array}$ \\
\hline Up & SNCA & 2 & $\begin{array}{l}\text { hsa-mir- } \\
153-3 p\end{array}$ & Down & HLA-DMB & 2 & $\begin{array}{c}\text { hsa-mir-9- } \\
5 \mathrm{p}\end{array}$ \\
\hline Up & FZD3 & 2 & $\begin{array}{c}\text { hsa-mir- } \\
31-5 p\end{array}$ & Down & IRF8 & 2 & $\begin{array}{l}\text { hsa-mir- } \\
646\end{array}$ \\
\hline Up & NME5 & 2 & $\begin{array}{l}\text { hsa-mir- } \\
148 b-3 p\end{array}$ & Down & IL13RA1 & 2 & $\begin{array}{c}\text { hsa-mir- } \\
143-3 p\end{array}$ \\
\hline Up & CABP1 & 2 & $\begin{array}{l}\text { hsa-mir- } \\
124-3 p\end{array}$ & Down & KCNMA1 & 2 & $\begin{array}{l}\text { hsa-mir- } \\
211-5 p\end{array}$ \\
\hline
\end{tabular}




\begin{tabular}{|c|c|c|c|c|c|c|c|}
\hline Up & ARHGAP44 & 2 & $\begin{array}{l}\text { hsa-mir- } \\
30 c-5 p\end{array}$ & Down & LYL1 & 2 & $\begin{array}{c}\text { hsa-mir- } \\
10 \mathrm{~b}-5 \mathrm{p}\end{array}$ \\
\hline Up & WDR7 & 2 & $\begin{array}{c}\text { hsa-mir- } \\
18 \mathrm{a}-3 \mathrm{p}\end{array}$ & Down & MT1X & 2 & $\begin{array}{l}\text { hsa-mir- } \\
1225-3 p\end{array}$ \\
\hline Up & SLC22A17 & 2 & $\begin{array}{l}\text { hsa-mir- } \\
3529-3 p\end{array}$ & Down & $\mathrm{PC}$ & 2 & $\begin{array}{l}\text { hsa-mir- } \\
125 a-5 p\end{array}$ \\
\hline Up & ATP6V1H & 2 & $\begin{array}{l}\text { hsa-mir- } \\
155-5 p\end{array}$ & Down & SERPINB6 & 2 & $\begin{array}{l}\text { hsa-mir- } \\
124-3 p\end{array}$ \\
\hline $\mathrm{Up}$ & DCAF6 & 2 & $\begin{array}{c}\text { hsa-let-7e- } \\
5 \mathrm{p}\end{array}$ & Down & MAPK4 & 2 & $\begin{array}{c}\text { hsa-mir- } \\
127-3 p\end{array}$ \\
\hline $\mathrm{Up}$ & NAPB & 2 & $\begin{array}{l}\text { hsa-mir- } \\
19 b-3 p\end{array}$ & Down & RYR3 & 2 & $\begin{array}{l}\text { hsa-mir- } \\
124-3 p\end{array}$ \\
\hline Up & DHRS11 & 2 & $\begin{array}{l}\text { hsa-mir- } \\
\text { 296-3p }\end{array}$ & Down & ITSN1 & 2 & $\begin{array}{l}\text { hsa-mir- } \\
\text { 296-3p }\end{array}$ \\
\hline $\mathrm{Up}$ & BEX2 & 2 & $\begin{array}{l}\text { hsa-mir- } \\
204-5 p\end{array}$ & Down & TBXAS1 & 2 & $\begin{array}{l}\text { hsa-mir- } \\
\text { 34b-3p }\end{array}$ \\
\hline $\mathrm{Up}$ & CEP41 & 2 & $\begin{array}{l}\text { hsa-mir- } \\
193 b-3 p\end{array}$ & Down & WFS1 & 2 & $\begin{array}{l}\text { hsa-mir- } \\
21-5 p\end{array}$ \\
\hline Up & SLITRK1 & 2 & $\begin{array}{l}\text { hsa-mir- } \\
24-1-5 p\end{array}$ & Down & AKR1C3 & 2 & $\begin{array}{l}\text { hsa-mir- } \\
155-5 \mathrm{p}\end{array}$ \\
\hline Up & ZNF385B & 2 & $\begin{array}{l}\text { hsa-mir- } \\
215-5 p\end{array}$ & Down & SLC16A3 & 2 & $\begin{array}{l}\text { hsa-mir- } \\
16-5 \mathrm{p}\end{array}$ \\
\hline $\mathrm{Up}$ & AMPH & 1 & $\begin{array}{c}\text { hsa-let-7b- } \\
5 \mathrm{p}\end{array}$ & Down & FAM189A2 & 2 & $\begin{array}{c}\text { hsa-mir- } \\
192-5 p\end{array}$ \\
\hline $\mathrm{Up}$ & ATP6V0C & 1 & $\begin{array}{c}\text { hsa-mir- } \\
421\end{array}$ & Down & BAG3 & 2 & $\begin{array}{l}\text { hsa-mir- } \\
221-3 p\end{array}$ \\
\hline Up & ATP6V1G2 & 1 & $\begin{array}{l}\text { hsa-mir- } \\
181 a-5 p\end{array}$ & Down & PRDX6 & 2 & $\begin{array}{l}\text { hsa-mir- } \\
632\end{array}$ \\
\hline Up & CAMK2B & 1 & $\begin{array}{l}\text { hsa-mir- } \\
186-5 p\end{array}$ & Down & IFITM2 & 2 & $\begin{array}{l}\text { hsa-let-7c- } \\
3 \mathrm{p}\end{array}$ \\
\hline $\mathrm{Up}$ & CDH18 & 1 & $\begin{array}{c}\text { hsa-let-7e- } \\
5 \mathrm{p}\end{array}$ & Down & VAMP5 & 2 & $\begin{array}{l}\text { hsa-mir-9- } \\
5 \mathrm{p}\end{array}$ \\
\hline
\end{tabular}




\begin{tabular}{|c|c|c|c|c|c|c|c|}
\hline Up & CDK5 & 1 & $\begin{array}{c}\text { hsa-mir- } \\
155-5 \mathrm{p}\end{array}$ & Down & CD300A & 2 & $\begin{array}{l}\text { hsa-mir- } \\
128-3 p\end{array}$ \\
\hline Up & CHGB & 1 & $\begin{array}{c}\text { hsa-mir- } \\
375\end{array}$ & Down & TMC6 & 2 & $\begin{array}{c}\text { hsa-mir- } \\
195-5 p\end{array}$ \\
\hline Up & CHRM1 & 1 & $\begin{array}{c}\text { hsa-mir- } \\
107\end{array}$ & Down & RGCC & 2 & $\begin{array}{l}\text { hsa-mir- } \\
26 \mathrm{~b}-5 \mathrm{p}\end{array}$ \\
\hline Up & DNM1 & 1 & $\begin{array}{c}\text { hsa-mir- } \\
615-3 p\end{array}$ & Down & NECAP2 & 2 & $\begin{array}{l}\text { hsa-mir- } \\
335-5 p\end{array}$ \\
\hline $\mathrm{Up}$ & DYNC1I1 & 1 & $\begin{array}{c}\text { hsa-mir- } \\
335-5 p\end{array}$ & Down & NDRG2 & 2 & $\begin{array}{c}\text { hsa-mir- } \\
650\end{array}$ \\
\hline Up & KIF3C & 1 & $\begin{array}{c}\text { hsa-mir- } \\
186-3 p\end{array}$ & Down & TMBIM1 & 2 & $\begin{array}{l}\text { hsa-mir- } \\
124-3 p\end{array}$ \\
\hline Up & NEFL & 1 & $\begin{array}{c}\text { hsa-mir- } \\
\text { 32-5p }\end{array}$ & Down & SLC25A18 & 2 & $\begin{array}{l}\text { hsa-mir- } \\
148 b-3 p\end{array}$ \\
\hline $\mathrm{Up}$ & NPY & 1 & $\begin{array}{c}\text { hsa-mir- } \\
335-5 p\end{array}$ & Down & RHPN2 & 2 & $\begin{array}{c}\text { hsa-mir- } \\
32-5 p\end{array}$ \\
\hline $\mathrm{Up}$ & PRKAR1B & 1 & $\begin{array}{c}\text { hsa-mir- } \\
218-5 p\end{array}$ & Down & EML3 & 2 & $\begin{array}{c}\text { hsa-mir-1- } \\
3 \mathrm{p}\end{array}$ \\
\hline Up & PRKCG & 1 & $\begin{array}{l}\text { hsa-mir- } \\
335-5 p\end{array}$ & Down & APOC1 & 1 & $\begin{array}{l}\text { hsa-mir- } \\
335-5 p\end{array}$ \\
\hline Up & PTPRN & 1 & $\begin{array}{c}\text { hsa-mir- } \\
18 \mathrm{a}-3 \mathrm{p}\end{array}$ & Down & AQP4 & 1 & $\begin{array}{l}\text { hsa-mir- } \\
320 \mathrm{a}\end{array}$ \\
\hline $\mathrm{Up}$ & PTPRR & 1 & $\begin{array}{c}\text { hsa-mir- } \\
150-5 p\end{array}$ & Down & ARHGDIB & 1 & $\begin{array}{l}\text { hsa-mir- } \\
\text { 34a-5p }\end{array}$ \\
\hline $\mathrm{Up}$ & PVALB & 1 & $\begin{array}{l}\text { hsa-mir- } \\
26 b-5 p\end{array}$ & Down & ATP1B2 & 1 & $\begin{array}{l}\text { hsa-mir- } \\
335-5 p\end{array}$ \\
\hline Up & SCN4B & 1 & $\begin{array}{c}\text { hsa-mir- } \\
122-5 p\end{array}$ & Down & C1QA & 1 & $\begin{array}{l}\text { hsa-mir- } \\
335-5 p\end{array}$ \\
\hline Up & SNRPN & 1 & $\begin{array}{l}\text { hsa-mir- } \\
26 b-5 p\end{array}$ & Down & C1QB & 1 & $\begin{array}{l}\text { hsa-mir- } \\
26 b-5 p\end{array}$ \\
\hline $\mathrm{Up}$ & STAT4 & 1 & $\begin{array}{c}\text { hsa-mir- } \\
141-3 p\end{array}$ & Down & CD14 & 1 & $\begin{array}{l}\text { hsa-mir- } \\
335-5 p\end{array}$ \\
\hline
\end{tabular}




\begin{tabular}{|c|c|c|c|c|c|c|c|}
\hline Up & SYN1 & 1 & $\begin{array}{c}\text { hsa-mir- } \\
335-5 p\end{array}$ & Down & CD37 & 1 & $\begin{array}{c}\text { hsa-mir- } \\
335-5 p\end{array}$ \\
\hline Up & SYN2 & 1 & $\begin{array}{l}\text { hsa-mir- } \\
335-5 p\end{array}$ & Down & CHI3L2 & 1 & $\begin{array}{l}\text { hsa-mir- } \\
335-5 p\end{array}$ \\
\hline Up & TARBP1 & 1 & $\begin{array}{c}\text { hsa-mir- } \\
124-3 p\end{array}$ & Down & CTSH & 1 & $\begin{array}{c}\text { hsa-mir- } \\
124-3 p\end{array}$ \\
\hline Up & SCG2 & 1 & $\begin{array}{c}\text { hsa-mir- } \\
98-5 p\end{array}$ & Down & CYP2J2 & 1 & $\begin{array}{c}\text { hsa-let-7b- } \\
5 \mathrm{p}\end{array}$ \\
\hline $\mathrm{Up}$ & USP11 & 1 & $\begin{array}{c}\text { hsa-mir- } \\
10 a-5 p\end{array}$ & Down & DTNA & 1 & $\begin{array}{c}\text { hsa-mir- } \\
8485\end{array}$ \\
\hline Up & CNTNAP1 & 1 & $\begin{array}{l}\text { hsa-mir- } \\
132-3 p\end{array}$ & Down & TYMP & 1 & $\begin{array}{l}\text { hsa-mir- } \\
92 a-3 p\end{array}$ \\
\hline Up & CDK5R2 & 1 & $\begin{array}{l}\text { hsa-mir- } \\
106 b-5 p\end{array}$ & Down & EYA2 & 1 & $\begin{array}{c}\text { hsa-mir- } \\
\text { 30a-5p }\end{array}$ \\
\hline Up & SYNGR3 & 1 & $\begin{array}{l}\text { hsa-mir- } \\
335-5 p\end{array}$ & Down & FBLN1 & 1 & $\begin{array}{l}\text { hsa-mir- } \\
\text { 30a-3p }\end{array}$ \\
\hline $\mathrm{Up}$ & SLIT2 & 1 & $\begin{array}{l}\text { hsa-mir- } \\
\text { 330-3p }\end{array}$ & Down & FCER1G & 1 & $\begin{array}{l}\text { hsa-mir- } \\
1225-3 p\end{array}$ \\
\hline Up & CACNG3 & 1 & $\begin{array}{l}\text { hsa-mir- } \\
26 b-5 p\end{array}$ & Down & FCGRT & 1 & $\begin{array}{c}\text { hsa-mir- } \\
\text { 335-5p }\end{array}$ \\
\hline $\mathrm{Up}$ & CAP2 & 1 & $\begin{array}{l}\text { hsa-mir- } \\
605-5 p\end{array}$ & Down & GFAP & 1 & $\begin{array}{l}\text { hsa-mir- } \\
335-5 p\end{array}$ \\
\hline Up & ARPP21 & 1 & $\begin{array}{c}\text { hsa-mir- } \\
124-3 p\end{array}$ & Down & HCLS1 & 1 & $\begin{array}{c}\text { hsa-mir- } \\
484\end{array}$ \\
\hline Up & PPM1E & 1 & $\begin{array}{l}\text { hsa-mir- } \\
335-5 p\end{array}$ & Down & HLA-DMA & 1 & $\begin{array}{l}\text { hsa-mir- } \\
\text { 335-5p }\end{array}$ \\
\hline Up & OPN3 & 1 & $\begin{array}{c}\text { hsa-mir- } \\
183-5 p\end{array}$ & Down & HLA-DPA1 & 1 & $\begin{array}{c}\text { hsa-mir- } \\
155-5 \mathrm{p}\end{array}$ \\
\hline Up & CNRIP1 & 1 & $\begin{array}{l}\text { hsa-mir- } \\
221-3 p\end{array}$ & Down & PRMT2 & 1 & $\begin{array}{c}\text { hsa-mir- } \\
17-3 p\end{array}$ \\
\hline Up & GLS2 & 1 & $\begin{array}{c}\text { hsa-mir- } \\
335-5 p\end{array}$ & Down & ITGAX & 1 & $\begin{array}{l}\text { hsa-mir- } \\
26 b-5 p\end{array}$ \\
\hline
\end{tabular}




\begin{tabular}{|c|c|c|c|c|c|c|c|}
\hline $\mathrm{U}$ & PCSK1N & 1 & $\begin{array}{l}\text { hsa-mir- } \\
31-5 p\end{array}$ & Down & LGALS9 & 1 & $\begin{array}{l}\text { hsa-mir- } \\
335-5 p\end{array}$ \\
\hline $\mathrm{U}$ & TAGLN3 & 1 & $\begin{array}{l}\text { hsa-mir- } \\
\text { 26b-5p }\end{array}$ & Down & MAOA & 1 & $\begin{array}{l}\text { hsa-mir- } \\
22-3 p\end{array}$ \\
\hline $\mathrm{U}$ & CA10 & 1 & $\begin{array}{l}\text { hsa-mir- } \\
148 b-3 p\end{array}$ & Down & MT1M & 1 & $\begin{array}{l}\text { hsa-mir- } \\
26 \mathrm{~b}-5 \mathrm{p}\end{array}$ \\
\hline $\mathrm{U}$ & PRDM8 & 1 & $\begin{array}{c}\text { hsa-mir-7- } \\
5 \mathrm{p}\end{array}$ & Down & MYT1 & 1 & $\begin{array}{l}\text { hsa-mir- } \\
27 a-3 p\end{array}$ \\
\hline $\mathrm{U}$ & SLC17A6 & 1 & $\begin{array}{l}\text { hsa-mir- } \\
26 \mathrm{~b}-5 \mathrm{p}\end{array}$ & Down & NDP & 1 & $\begin{array}{l}\text { hsa-mir- } \\
335-5 p\end{array}$ \\
\hline $\mathrm{U}$ & LIN7B & 1 & $\begin{array}{l}\text { hsa-mir- } \\
106 b-5 p\end{array}$ & Down & PLCD1 & 1 & $\begin{array}{l}\text { hsa-mir- } \\
335-5 p\end{array}$ \\
\hline $\mathrm{U}$ & WDR54 & 1 & $\begin{array}{l}\text { hsa-mir- } \\
23 \mathrm{~b}-3 \mathrm{p}\end{array}$ & Down & PLTP & 1 & $\begin{array}{l}\text { hsa-mir- } \\
\text { 335-5p }\end{array}$ \\
\hline $\mathrm{U}$ & CORO6 & 1 & $\begin{array}{c}\text { hsa-mir-7- } \\
5 \mathrm{p}\end{array}$ & Down & PLXNB1 & 1 & $\begin{array}{l}\text { hsa-mir- } \\
214-3 p\end{array}$ \\
\hline $\mathrm{U}$ & TOX2 & 1 & $\begin{array}{l}\text { hsa-mir- } \\
335-5 p\end{array}$ & Down & PMP2 & 1 & $\begin{array}{c}\text { hsa-mir-7- } \\
5 \mathrm{p}\end{array}$ \\
\hline $\mathrm{U}$ & MRAP2 & 1 & $\begin{array}{l}\text { hsa-mir- } \\
21-5 \mathrm{p}\end{array}$ & Down & PPP1R3C & 1 & $\begin{array}{l}\text { hsa-mir- } \\
335-5 \mathrm{p}\end{array}$ \\
\hline $\mathrm{U}$ & GPRASP2 & 1 & $\begin{array}{l}\text { hsa-mir- } \\
744-5 p\end{array}$ & Down & PTPN6 & 1 & $\begin{array}{l}\text { hsa-mir- } \\
1225-3 p\end{array}$ \\
\hline $\mathrm{U}$ & VSTM2L & 1 & $\begin{array}{l}\text { hsa-mir- } \\
335-5 p\end{array}$ & Down & RAB3IL1 & 1 & $\begin{array}{l}\text { hsa-mir- } \\
124-3 p\end{array}$ \\
\hline $\mathrm{U}$ & TMEM155 & 1 & $\begin{array}{l}\text { hsa-mir- } \\
335-5 p\end{array}$ & Down & RAC2 & 1 & $\begin{array}{l}\text { hsa-mir- } \\
193 b-3 p\end{array}$ \\
\hline $\mathrm{U}$ & SLC32A1 & 1 & $\begin{array}{l}\text { hsa-mir- } \\
148 b-3 p\end{array}$ & Down & RARRES2 & 1 & $\begin{array}{l}\text { hsa-mir- } \\
\text { 335-5p }\end{array}$ \\
\hline $\mathrm{U}$ & CBLN4 & 1 & $\begin{array}{c}\text { hsa-mir- } \\
335-5 p\end{array}$ & Down & RARRES3 & 1 & $\begin{array}{c}\text { hsa-mir- } \\
30 \mathrm{e}-3 \mathrm{p}\end{array}$ \\
\hline 0 & $\mathrm{ZCCHC} 12$ & 1 & $\begin{array}{c}\text { hsa-mir- } \\
615-3 p\end{array}$ & Down & RGS1 & 1 & $\begin{array}{l}\text { hsa-mir- } \\
374 a-5 p\end{array}$ \\
\hline
\end{tabular}




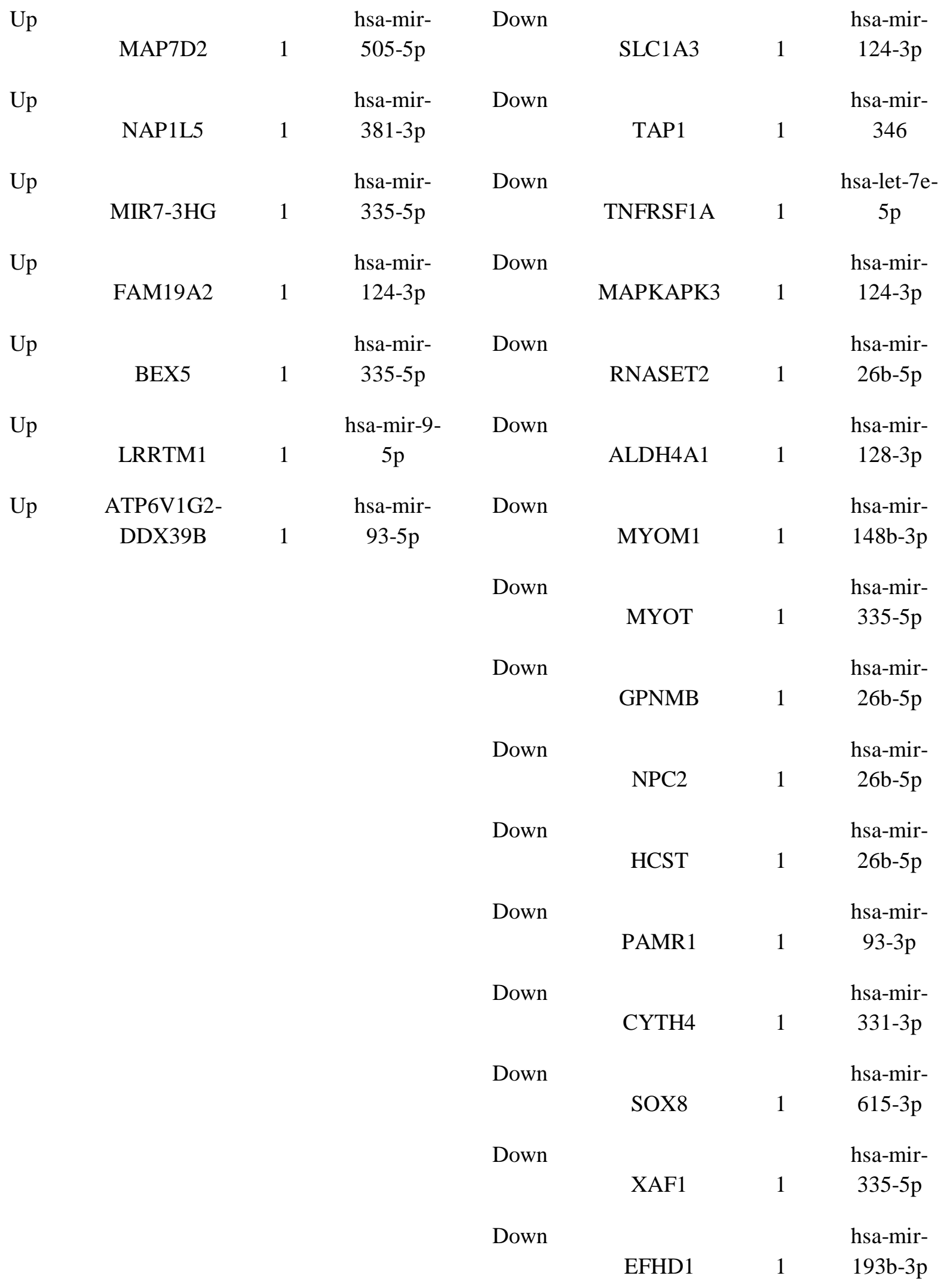


Down

Down

Down

Down

Down hsa-mir-7-

TRIM47

1 $5 \mathrm{p}$

hsa-mir-

CMTM7 1 124-3p

NAPSB

1

hsa-mir-

335-5p

hsa-mir-

SLC9A9

1

$124-3 p$

hsa-mir-

BTBD17 $1335-5 p$

Degree - No of miRNA interact with target gene. We taken any one miRNA in table.

Table 8 TF - target gene interaction table

\begin{tabular}{cccccccc}
$\begin{array}{c}\text { Regulatio } \\
\text { n }\end{array}$ & TF & $\begin{array}{c}\text { Degre } \\
\mathbf{e}\end{array}$ & Target Gene & $\begin{array}{c}\text { Regulatio } \\
\mathbf{n}\end{array}$ & TF & $\begin{array}{c}\text { Degre } \\
\mathbf{e}\end{array}$ & Target Gene \\
\hline Up & SOX2 & 235 & CDK14 & Down & SOX2 & 224 & HSPA1A \\
Up & MYC & 193 & YWHAH & Down & SPI1 & 212 & CD300A \\
Up & REST & 182 & SYN2 & Down & EGR1 & 197 & HLA-DOA \\
Up & SUZ12 & 179 & SYT1 & Down & HNF4A & 184 & TST \\
Up & AR & 169 & MICAL2 & Down & MYC & 179 & HSPB1 \\
Up & STAT3 & 169 & OAT & Down & RUNX1 & 174 & COX19 \\
Up & NANOG & 166 & SYNGR1 & Down & TP63 & 171 & HMG20B \\
Up & TP53 & 161 & VGF & Down & FLI1 & 168 & GPC5 \\
Up & RUNX1 & 156 & TPM1 & Down & NANOG & 165 & LY96 \\
Up & SMAD4 & 155 & ATP2A2 & Down & STAT3 & 163 & ABCA1 \\
Up & POU5F1 & 154 & STX1A & Down & MITF & 155 & BCL6 \\
Up & TP63 & 140 & ICA1 & Down & AR & 154 & TSPO \\
Up & HNF4A & 136 & SV2B & Down & TP53 & 153 & CRYAB \\
Up & SPI1 & 135 & SVOP & Down & POU5F1 & 149 & METTL7B
\end{tabular}


medRxiv preprint doi: https://doi.org/10.1101/2020.12.21.20248688; this version posted December 24, 2020. The copyright holder for this preprint (which was not certified by peer review) is the author/funder, who has granted medRxiv a license to display the preprint in perpetuity. All rights reserved. No reuse allowed without permission.

\begin{tabular}{|c|c|c|c|c|c|c|c|}
\hline $\mathrm{Up}$ & CREM & 132 & РPP3CB & Down & GATA2 & 146 & NPC2 \\
\hline Up & FLI1 & 129 & ATP6V1G2 & Down & GATA1 & 133 & SMOX \\
\hline Up & TCF4 & 127 & NGEF & Down & PPARG & 126 & TBL1X \\
\hline Up & MITF & 127 & STXBP1 & Down & E2F1 & 119 & MAPKAPK3 \\
\hline Up & MTF2 & 124 & GABRA1 & Down & KLF4 & 115 & INPPL1 \\
\hline Up & $\mathrm{E} 2 \mathrm{~F} 1$ & 117 & SNAP25 & Down & SUZ12 & 115 & CYBA \\
\hline Up & SMARCA4 & 110 & MFSD6 & Down & TFAP2C & 112 & TNFRSF1A \\
\hline Up & SIN3B & 104 & ATP6V1H & Down & FOXA2 & 106 & TBXAS1 \\
\hline Up & CREB1 & 103 & ATP6V1E1 & Down & SMAD4 & 102 & LAPTM5 \\
\hline Up & RCOR3 & 103 & HPCA & Down & SOX9 & 102 & RAB31 \\
\hline $\mathrm{Up}$ & TRIM28 & 101 & SYN1 & Down & TCF3 & 100 & HLA-DRA \\
\hline Up & RUNX2 & 99 & RUNDC3A & Down & SMARCA4 & 99 & MYO10 \\
\hline Up & TCF3 & 98 & NT5DC3 & Down & TAL1 & 97 & VAMP8 \\
\hline Up & PPARG & 98 & PFN2 & Down & TRIM28 & 94 & CD44 \\
\hline Up & PPARD & 96 & CNTNAP2 & Down & TCF4 & 93 & CCND1 \\
\hline Up & KLF4 & 93 & GNAO1 & Down & REST & 90 & EXOG \\
\hline Up & GATA1 & 93 & TUBA1B & Down & PPARD & 90 & MYOM1 \\
\hline Up & GATA2 & 91 & VSTM2L & Down & SETDB1 & 88 & HOXB6 \\
\hline Up & SALL4 & 88 & EPDR1 & Down & SALL4 & 86 & DDIT4L \\
\hline Up & TET1 & 85 & RAP1GAP2 & Down & SRY & 85 & NEK6 \\
\hline Up & SETDB1 & 82 & DLX1 & Down & ESR1 & 85 & SERPINA3 \\
\hline Up & TFAP2C & 82 & AFTPH & Down & MECOM & 85 & CSK \\
\hline Up & KDM5B & 80 & YWHAZ & Down & TET1 & 83 & TLR5 \\
\hline Up & SMAD3 & 80 & TOX2 & Down & EP300 & 83 & ATP6V0E1 \\
\hline Up & EP300 & 78 & TUBB3 & Down & RUNX2 & 80 & GYPC \\
\hline Up & FOXP1 & 77 & GOLGA8B & Down & MTF2 & 78 & RASL12 \\
\hline
\end{tabular}




\begin{tabular}{|c|c|c|c|c|c|c|c|}
\hline $\mathrm{Up}$ & SOX9 & 76 & RELL2 & Down & SMAD3 & 77 & RARRES2 \\
\hline Up & SRY & 76 & SULT4A1 & Down & SIN3B & 76 & EZR \\
\hline $\mathrm{Up}$ & OLIG2 & 76 & DYNC1I1 & Down & FOXP1 & 76 & SIGLEC10 \\
\hline Up & TAL1 & 75 & LDOC1 & Down & CREM & 74 & ADD3 \\
\hline Up & YAP1 & 75 & SYP & Down & ASH2L & 73 & APOE \\
\hline Up & TEAD4 & 74 & AK5 & Down & WT1 & 73 & $\mathrm{ZCCHC} 24$ \\
\hline $\mathrm{Up}$ & & & & Down & & & HIST2H2AA \\
\hline & $\mathrm{EZH} 2$ & 72 & DPP10 & & CREB1 & 71 & 3 \\
\hline Up & BMI1 & 71 & FGF12 & Down & CUX1 & 70 & UNC93B1 \\
\hline Up & FOXA2 & 69 & CAMK1D & Down & EOMES & 69 & AQP4 \\
\hline Up & EOMES & 68 & ENSA & Down & KLF1 & 68 & APOC2 \\
\hline Up & RNF2 & 67 & NELL2 & Down & ERG & 67 & VEGFB \\
\hline Up & ESR1 & 65 & RGS7 & Down & RCOR3 & 66 & SP110 \\
\hline Up & ZNF281 & 63 & MLLT11 & Down & SCLY & 66 & ALOX5AP \\
\hline Up & NR3C1 & 63 & NPTXR & Down & ZNF281 & 64 & RHOBTB3 \\
\hline Up & TFAP2A & 63 & MEG3 & Down & TFCP2L1 & 64 & PPP1R3C \\
\hline Up & JARID2 & 62 & LRRTM1 & Down & ATF3 & 64 & AXL \\
\hline Up & CUX1 & 61 & PRDM8 & Down & YAP1 & 62 & RHOC \\
\hline Up & $\mathrm{E} 2 \mathrm{~F} 4$ & 59 & DDX24 & Down & NR3C1 & 58 & MAOA \\
\hline Up & WT1 & 58 & ETS2 & Down & ZNF217 & 55 & ZFHX3 \\
\hline Up & ZNF217 & 57 & RTN4 & Down & TFAP2A & 55 & MT1X \\
\hline Up & ASH2L & 57 & PAK1 & Down & RELA & 55 & EBI3 \\
\hline Up & ESRRB & 57 & FBXO34 & Down & NFE2L2 & 54 & CTSC \\
\hline Up & DMRT1 & 55 & CEP41 & Down & SMAD2 & 54 & EMP1 \\
\hline Up & POU3F2 & 54 & TAC1 & Down & ZFX & 53 & TSC22D4 \\
\hline Up & MYCN & 53 & CPLX2 & Down & DMRT1 & 53 & WFS1 \\
\hline
\end{tabular}




\begin{tabular}{|c|c|c|c|c|c|c|c|}
\hline Up & KLF1 & 53 & IARS & Down & NR0B1 & 53 & C1QA \\
\hline $\mathrm{Up}$ & ERG & 52 & OPCML & Down & E2F4 & 52 & APBB1IP \\
\hline Up & TBX5 & 50 & PVALB & Down & PRDM14 & 52 & GPR37L1 \\
\hline Up & JUN & 49 & GOT1 & Down & GATA4 & 52 & NQO1 \\
\hline $\mathrm{Up}$ & SCLY & 48 & CCNA1 & Down & BACH1 & 52 & DOCK2 \\
\hline Up & YY1 & 48 & ATP6V0C & Down & LMO2 & 51 & MAPK4 \\
\hline Up & ZFX & 47 & CMAS & Down & RBPJ & 51 & TEAD2 \\
\hline Up & SMAD2 & 46 & $\begin{array}{c}\text { GABARAPL } \\
1\end{array}$ & Down & STAT4 & 50 & CEBPD \\
\hline $\mathrm{Up}$ & RAD21 & 45 & BCL11A & Down & KDM5B & 49 & ATXN3 \\
\hline Up & CTNNB1 & 45 & RBFOX2 & Down & GFI1B & 49 & LYL1 \\
\hline Up & SOX17 & 44 & APP & Down & TEAD4 & 48 & HTRA1 \\
\hline Up & PRDM14 & 44 & TOMM20 & Down & TCF7 & 48 & LAT2 \\
\hline Up & FOXP2 & 44 & STAMBPL1 & Down & FOXP2 & 47 & MYT1 \\
\hline Up & NR0B1 & 44 & TAGLN3 & Down & RAD21 & 47 & CЕВPB \\
\hline Up & ATF3 & 43 & UCHL1 & Down & MEIS1 & 46 & EYA2 \\
\hline Up & SIN3A & 43 & CAMTA1 & Down & NR1H3 & 46 & LY86 \\
\hline Up & NFE2L2 & 42 & ADAM23 & Down & $\mathrm{EZH} 2$ & 45 & ITGB4 \\
\hline Up & FOXO3 & 42 & VAMP1 & Down & VDR & 44 & IL17RB \\
\hline Up & LMO2 & 42 & FKBP1A & Down & MYCN & 44 & DCLRE1C \\
\hline $\mathrm{Up}$ & BACH1 & 42 & AP3B2 & Down & MYB & 44 & IFNGR1 \\
\hline $\mathrm{Up}$ & VDR & 41 & CD200 & Down & JUN & 43 & RANBP3L \\
\hline Up & HOXC9 & 40 & ATCAY & Down & PBX1 & 42 & $\mathrm{PC}$ \\
\hline Up & TFCP2L1 & 39 & GAD2 & Down & MYBL2 & 41 & AIF1 \\
\hline Up & MECOM & 39 & EXTL2 & Down & RNF2 & 41 & OAF \\
\hline $\mathrm{Up}$ & EED & 39 & DPP10 & Down & OLIG2 & 40 & RPS27 \\
\hline
\end{tabular}




\begin{tabular}{|c|c|c|c|c|c|c|c|}
\hline Up & CEBPB & 39 & STAT4 & Down & FOXO3 & 39 & CDKN1A \\
\hline Up & PAX3 & 38 & CELF2 & Down & YY1 & 38 & FOXO4 \\
\hline Up & ELK1 & 38 & SST & Down & TBX3 & 37 & NPL \\
\hline $\mathrm{Up}$ & RCOR1 & 38 & EPB41L3 & Down & SOX17 & 35 & RAI14 \\
\hline Up & GFI1B & 37 & NECAP1 & Down & BMI1 & 35 & GJA1 \\
\hline Up & PBX1 & 37 & TMX4 & Down & HOXB4 & 34 & BAZ2B \\
\hline Up & CCND1 & 36 & WASF1 & Down & TBX5 & 34 & IRF8 \\
\hline $\mathrm{Up}$ & GATA4 & 36 & MAP2K4 & Down & CTNNB1 & 34 & MKNK2 \\
\hline Up & EWSR1 & 36 & BASP1 & Down & CTCF & 33 & DAB2 \\
\hline Up & PHC1 & 35 & INA & Down & ESRRB & 33 & DFFA \\
\hline Up & RBPJ & 33 & WDR54 & Down & NUCKS1 & 30 & NDRG2 \\
\hline Up & SOX11 & 32 & RNF41 & Down & HSF1 & 30 & FAM189A2 \\
\hline Up & XRN2 & 31 & AGK & Down & ESR2 & 30 & NDRG1 \\
\hline $\mathrm{Up}$ & MYBL2 & 31 & LIN7B & Down & ELF1 & 29 & MYL12A \\
\hline $\mathrm{Up}$ & MEF2A & 30 & KIAA0513 & Down & ELK1 & 27 & MOB3C \\
\hline Up & TTF2 & 29 & ACOT7 & Down & SOX11 & 27 & CD86 \\
\hline Up & TCF7 & 29 & DNM1 & Down & $\mathrm{CDX} 2$ & 27 & KCNMA1 \\
\hline Up & CTCF & 29 & FAM3C & Down & CNOT3 & 26 & WAS \\
\hline Up & $\mathrm{CDX} 2$ & 29 & MFSD6 & Down & JARID2 & 26 & CSF2RA \\
\hline Up & DNAJC2 & 29 & ATP2B3 & Down & NR1I2 & 26 & PDK4 \\
\hline Up & DACH1 & 28 & ACP1 & Down & CLOCK & 26 & DNAJB6 \\
\hline $\mathrm{Up}$ & CNOT3 & 28 & CYFIP2 & Down & DACH1 & 25 & NKAIN4 \\
\hline $\mathrm{Up}$ & ZFP42 & 28 & USP11 & Down & RCOR1 & 25 & $\mathrm{DIO} 2$ \\
\hline Up & TBX3 & 27 & RBFOX1 & Down & ARNT & 24 & LHFPL2 \\
\hline Up & MEIS1 & 27 & TUSC3 & Down & SRF & 24 & TROVE2 \\
\hline $\mathrm{Up}$ & ARNT & 25 & SPHKAP & Down & EWSR1 & 24 & RRBP1 \\
\hline
\end{tabular}




\begin{tabular}{|c|c|c|c|c|c|c|c|}
\hline Up & ELF5 & 24 & MIR7-3HG & Down & TTF2 & 23 & BCAN \\
\hline $\mathrm{Up}$ & PDX1 & 24 & OPN3 & Down & XRN2 & 23 & TPP1 \\
\hline Up & HOXB4 & 22 & NME5 & Down & MEF2A & 23 & DBI \\
\hline Up & SREBF2 & 22 & SYBU & Down & FOXP3 & 22 & СMTM3 \\
\hline Up & PRDM5 & 22 & CDK5 & Down & PHF8 & 22 & C1ORF198 \\
\hline Up & NR1I2 & 22 & CALY & Down & ZFP42 & 22 & HAP1 \\
\hline Up & LYL1 & 21 & FBXL2 & Down & SIN3A & 22 & STAT5A \\
\hline Up & SRF & 20 & MAPK9 & Down & TBP & 22 & S100A1 \\
\hline Up & FOXP3 & 19 & PRKCE & Down & DNAJC2 & 22 & OAF \\
\hline Up & NACC1 & 19 & NSF & Down & HOXC9 & 21 & SLC7A2 \\
\hline $\mathrm{Up}$ & PHF8 & 18 & SLC38A1 & Down & TCF7L2 & 21 & SLC11A1 \\
\hline Up & MYB & 18 & PFN2 & Down & SREBF1 & 21 & LPP \\
\hline Up & NUCKS1 & 18 & EGR1 & Down & STAT1 & 20 & ALDH7A1 \\
\hline Up & RELA & 18 & ETS2 & Down & IRF1 & 20 & SASH3 \\
\hline Up & ELF1 & 18 & KRT222 & Down & AHR & 19 & SRGAP1 \\
\hline Up & IRF8 & 18 & ARPP21 & Down & PAX3 & 19 & MEGF10 \\
\hline Up & THAP11 & 18 & NDRG3 & Down & $\mathrm{PHC} 1$ & 19 & MSX1 \\
\hline Up & DROSHA & 18 & PRKAR1B & Down & SMAD1 & 19 & PAQR8 \\
\hline Up & GBX2 & 18 & NELL2 & Down & TAF7L & 18 & NUPR1 \\
\hline Up & ETS1 & 17 & NME1 & Down & GATA3 & 18 & PDCD4 \\
\hline Up & STAT5A & 17 & XK & Down & CRX & 18 & ATP1B2 \\
\hline Up & SMAD1 & 17 & DENND5B & Down & STAT6 & 18 & RGCC \\
\hline Up & PADI4 & 17 & ARHGAP32 & Down & RCOR2 & 18 & NCAN \\
\hline Up & GATA3 & 16 & NNAT & Down & RARG & 18 & AEBP1 \\
\hline Up & CRX & 16 & GPM6A & Down & CHD1 & 17 & PLIN3 \\
\hline Up & KDM5A & 15 & FAM216A & Down & POU3F2 & 17 & SPP1 \\
\hline
\end{tabular}




\begin{tabular}{|c|c|c|c|c|c|c|c|}
\hline Up & TCF7L2 & 15 & NSF & Down & NACC1 & 17 & DHRS3 \\
\hline Up & HTT & 15 & BEX2 & Down & SREBF2 & 17 & BRI3BP \\
\hline Up & CEBPD & 14 & CDH18 & Down & DROSHA & 17 & NWD1 \\
\hline Up & HSF1 & 14 & CCK & Down & ZIC3 & 16 & NINJ1 \\
\hline Up & NR4A2 & 14 & ITPR1 & Down & ASXL1 & 16 & SIPA1 \\
\hline Up & AHR & 13 & IGSF3 & Down & ELF5 & 14 & TMBIM1 \\
\hline Up & STAT1 & 13 & MOAP1 & Down & PRDM5 & 14 & CLIC1 \\
\hline Up & PAX6 & 13 & ADAM23 & Down & PDX1 & 14 & MYT1 \\
\hline Up & SREBF1 & 13 & MDH1 & Down & NFIB & 14 & APLNR \\
\hline Up & BCL3 & 12 & CORO6 & Down & HIF1A & 14 & ANGPTL4 \\
\hline Up & ASXL1 & 12 & UBE2N & Down & KDM5A & 13 & NPC2 \\
\hline Up & NFIB & 12 & AK5 & Down & EED & 13 & CD14 \\
\hline Up & CLOCK & 12 & BEX1 & Down & PADI4 & 13 & LCP1 \\
\hline Up & RCOR2 & 12 & PRSS3 & Down & PAX6 & 13 & SLC9A3R1 \\
\hline Up & TAF7L & 11 & ALDOA & Down & BCL3 & 12 & SDC4 \\
\hline Up & TFEB & 11 & FBXL2 & Down & TFEB & 12 & GPX1 \\
\hline $\mathrm{Up}$ & DCP1A & 11 & PNKD & Down & ETS1 & 10 & PAPOLA \\
\hline Up & STAT6 & 10 & CYFIP2 & Down & THAP11 & 10 & GPT2 \\
\hline Up & ESR2 & 10 & ZNF365 & Down & DCP1A & 9 & MT1E \\
\hline Up & HIF1A & 10 & C3ORF14 & Down & FOXO1 & 8 & ASAP3 \\
\hline Up & CHD1 & 9 & ACP1 & Down & ZNF263 & 8 & EBI3 \\
\hline Up & ZNF274 & 9 & STEAP2 & Down & NOTCH1 & 7 & FGR \\
\hline Up & AP1S2 & 8 & OCIAD1 & Down & HTT & 6 & DTNA \\
\hline Up & TBP & 8 & SLIT2 & Down & IKZF1 & 6 & $\mathrm{ZCCHC} 24$ \\
\hline $\mathrm{Up}$ & ZIC3 & 8 & ARPP21 & Down & HOXD13 & 5 & LEMD3 \\
\hline $\mathrm{Up}$ & NR1H3 & 8 & HENMT1 & Down & NR4A2 & 5 & LILRB3 \\
\hline
\end{tabular}




\begin{tabular}{|c|c|c|c|c|c|c|c|}
\hline Up & KDM6A & 7 & NT5DC3 & Down & ZNF274 & 5 & SYNJ2BP \\
\hline $\mathrm{Up}$ & IRF1 & 7 & DCAF6 & Down & GBX2 & 4 & RYR3 \\
\hline $\mathrm{Up}$ & HOXD13 & 7 & NPTN & Down & FOXM1 & 4 & DCLRE1C \\
\hline $\mathrm{Up}$ & IKZF1 & 7 & SNCA & Down & CIITA & 4 & PARVG \\
\hline Up & CEBPA & 6 & MAGED1 & Down & KLF2 & 3 & KANK1 \\
\hline \multirow[t]{2}{*}{ Up } & CDKN2AI & & & Down & & & \\
\hline & $\mathrm{P}$ & 6 & CALY & & KLF5 & 3 & SERPINA3 \\
\hline Up & PRDM16 & 6 & SLC25A12 & Down & AP1S2 & 3 & LCP1 \\
\hline Up & FOXO1 & 5 & KALRN & Down & KDM6A & 3 & FCGRT \\
\hline Up & SALL1 & 5 & RTN4 & Down & ZNF652 & 3 & TNFRSF1A \\
\hline Up & FOXM1 & 4 & TSPYL2 & Down & TCF21 & 3 & STK3 \\
\hline Up & GLI1 & 4 & ERC2 & Down & ETS2 & 2 & EMX2 \\
\hline Up & NOTCH1 & 3 & TUBB2A & Down & MYBL1 & 2 & TEAD2 \\
\hline \multirow[t]{2}{*}{ Up } & & & GABARAPL & Down & & & \\
\hline & RARG & 3 & 1 & & THRA & 2 & HTRA1 \\
\hline \multirow[t]{2}{*}{$\mathrm{Up}$} & & & & Down & CDKN2AI & & \\
\hline & THRA & 3 & CRYM & & $\mathrm{P}$ & 2 & ATP6V0E1 \\
\hline $\mathrm{Up}$ & CHD7 & 3 & PRKCG & Down & CHD7 & 2 & ITSN1 \\
\hline $\mathrm{Up}$ & HCFC1 & 2 & ARF3 & Down & PRDM16 & 2 & LHPP \\
\hline Up & ZNF652 & 2 & LAMP5 & Down & GLI1 & 2 & PLEKHB1 \\
\hline Up & TCF21 & 2 & ALDOA & Down & HCFC1 & 1 & DFFA \\
\hline Up & MYBL1 & 1 & MOAP1 & Down & ZNF322 & 1 & BBOX1 \\
\hline $\mathrm{Up}$ & ZNF322 & 1 & PTPRM & Down & BCL11B & 1 & SP110 \\
\hline $\mathrm{Up}$ & ZNF263 & 1 & WDR54 & Down & BP1 & 1 & LAPTM5 \\
\hline Up & BCL11B & 1 & TMX4 & & & & \\
\hline
\end{tabular}

\section{Figures}


medRxiv preprint doi: https://doi.org/10.1101/2020.12.21.20248688; this version posted December 24, 2020. The copyright holder for this preprint (which was not certified by peer review) is the author/funder, who has granted medRxiv a license to display the preprint in perpetuity.

All rights reserved. No reuse allowed without permission.

\section{A}

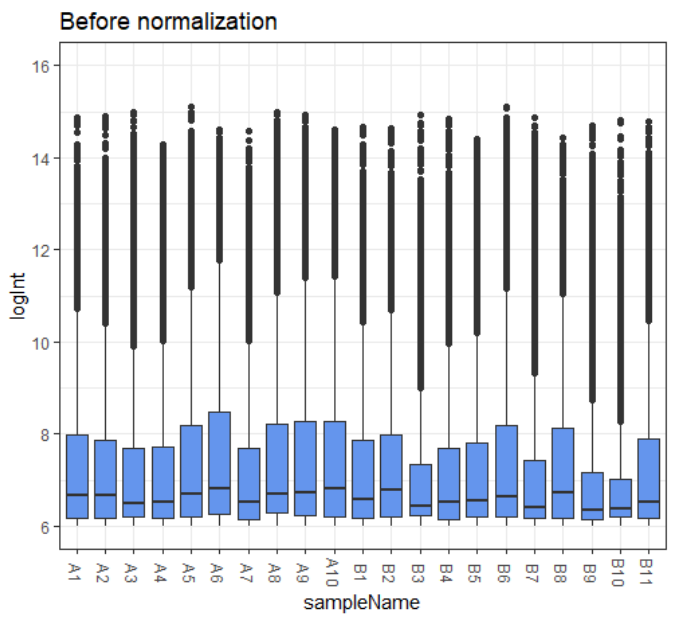

B

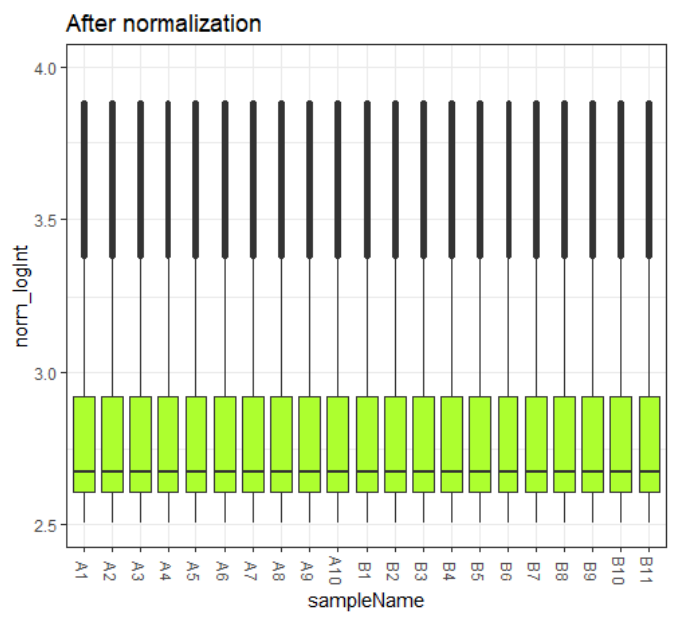

Fig. 1. Box plots of the gene expression data before normalization (A) and after normalization (B). Horizontal axis represents the sample symbol and the vertical axis represents the gene expression values. The black line in the box plot represents the median value of gene expression. (A1, A2, A3, A4, A5, A6, A7, A8, A9, A10 = normal controls samples; B1,B2, B3, B4,B5, B6, B7,B8, B9, B10 = sCJD samples) 
medRxiv preprint doi: https://doi.org/10.1101/2020.12.21.20248688; this version posted December 24, 2020. The copyright holder for this preprint (which was not certified by peer review) is the author/funder, who has granted medRxiv a license to display the preprint in perpetuity.

All rights reserved. No reuse allowed without permission.

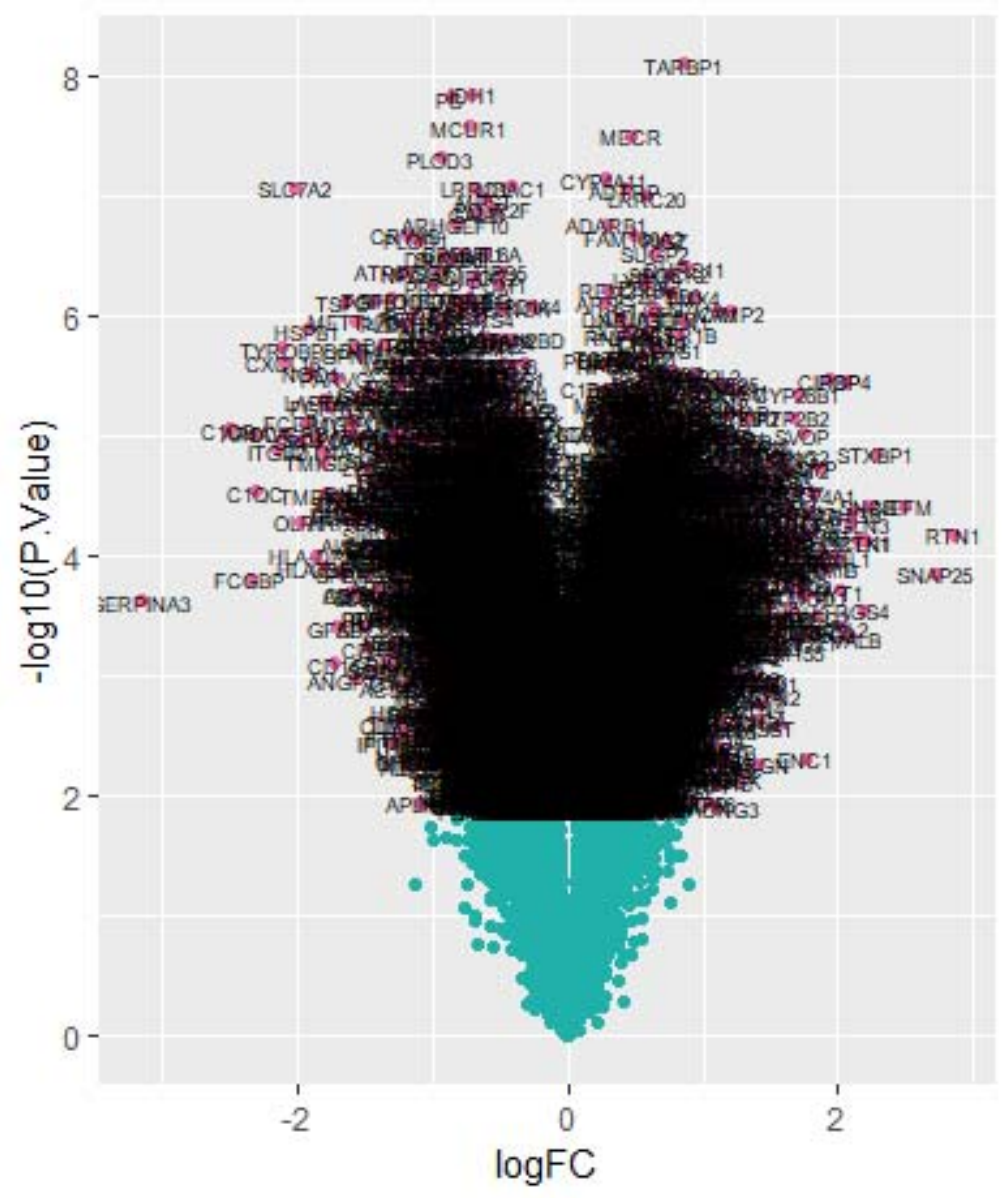

sig

- FDR $<0.05$

- Not Sig

Fig. 2. Volcano plot of differentially expressed genes. Genes with a significant change of more than two-fold were selected. 
medRxiv preprint doi: https://doi.org/10.1101/2020.12.21.20248688; this version posted December 24, 2020. The copyright holder for this preprint (which was not certified by peer review) is the author/funder, who has granted medRxiv a license to display the preprint in perpetuity.

All rights reserved. No reuse allowed without permission.
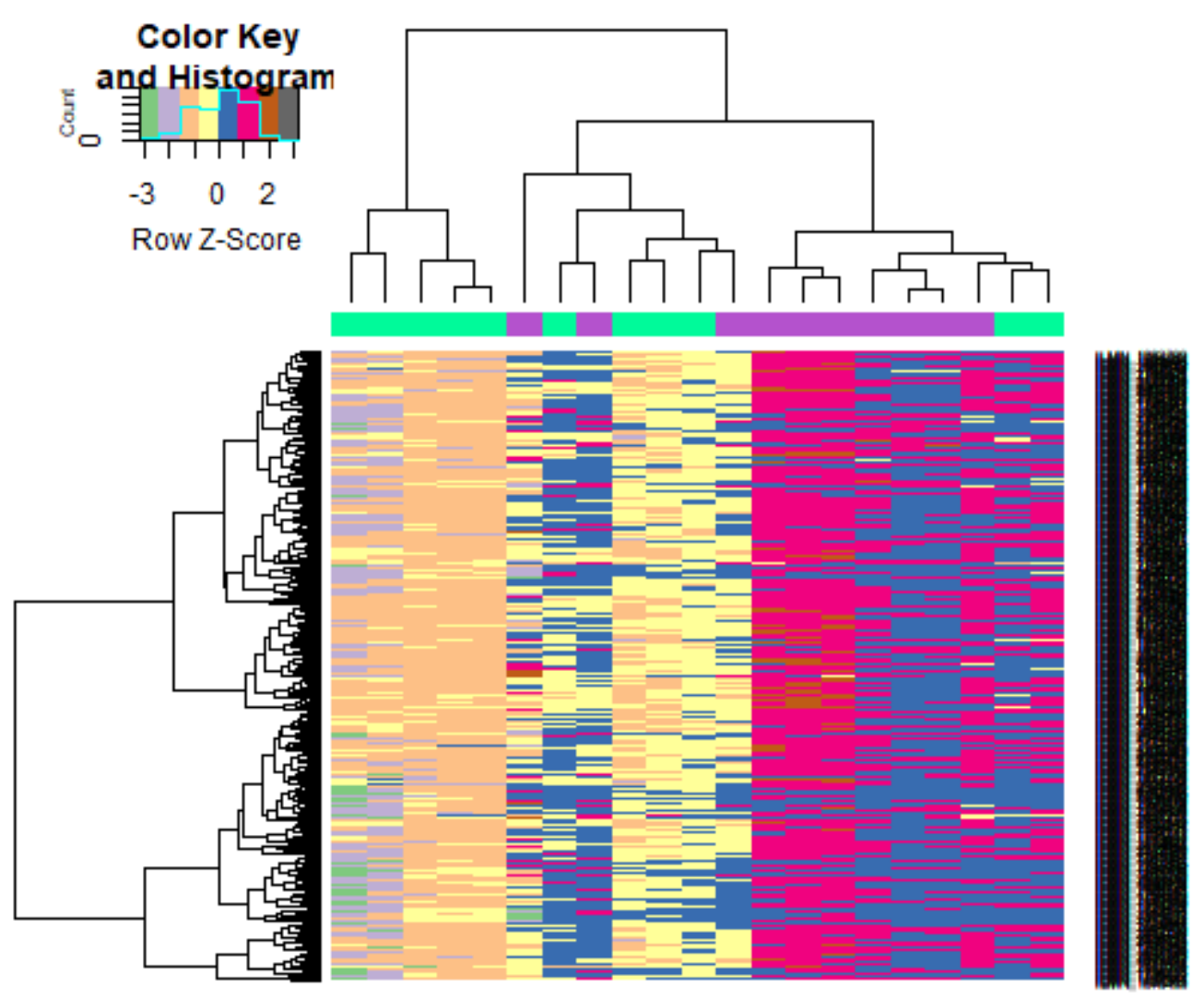

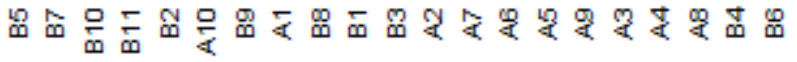

Fig. 3. Heat map of up regulated differentially expressed genes. Legend on the top left indicate log fold change of genes. (A1, A2, A3, A4, A5, A6, A7, A8, A9, A10 = normal controls samples; B1,B2, B3, B4,B5, B6, B7,B8, B9, $\mathrm{B} 10=$ sCJD samples) 
medRxiv preprint doi: https://doi.org/10.1101/2020.12.21.20248688; this version posted December 24, 2020. The copyright holder for this preprint (which was not certified by peer review) is the author/funder, who has granted medRxiv a license to display the preprint in perpetuity.

All rights reserved. No reuse allowed without permission.
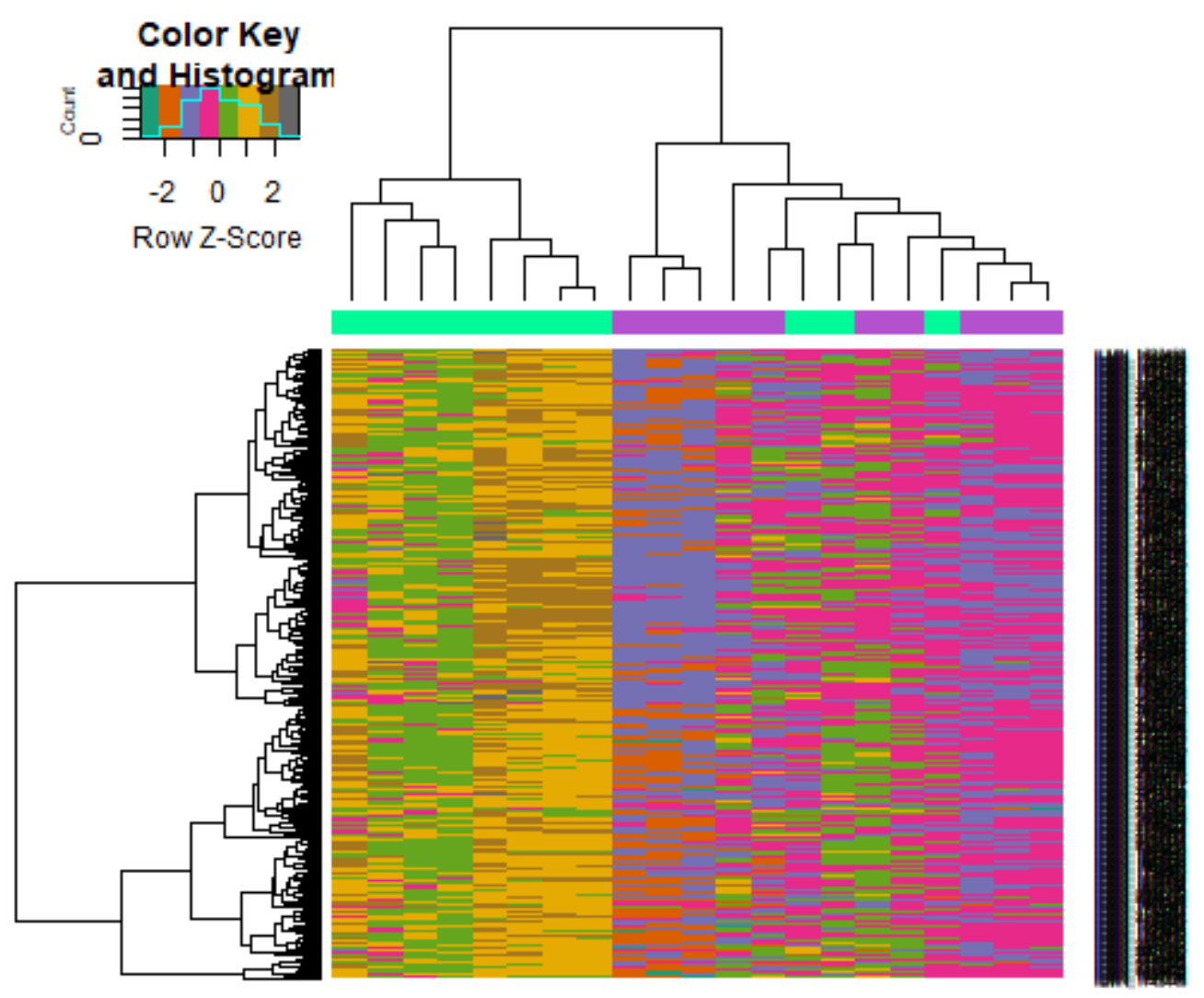

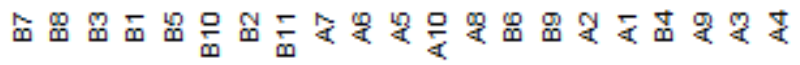

Fig. 4. Heat map of down regulated differentially expressed genes. Legend on the top left indicate log fold change of genes. (A1, A2, A3, A4, A5, A6, A7, A8, A9, A10 = normal controls samples; B1,B2, B3, B4,B5, B6, B7,B8, B9, $\mathrm{B} 10=$ sCJD samples) 
medRxiv preprint doi: https://doi.org/10.1101/2020.12.21.20248688; this version posted December 24, 2020. The copyright holder for this preprint (which was not certified by peer review) is the author/funder, who has granted medRxiv a license to display the preprint in perpetuity. All rights reserved. No reuse allowed without permission.

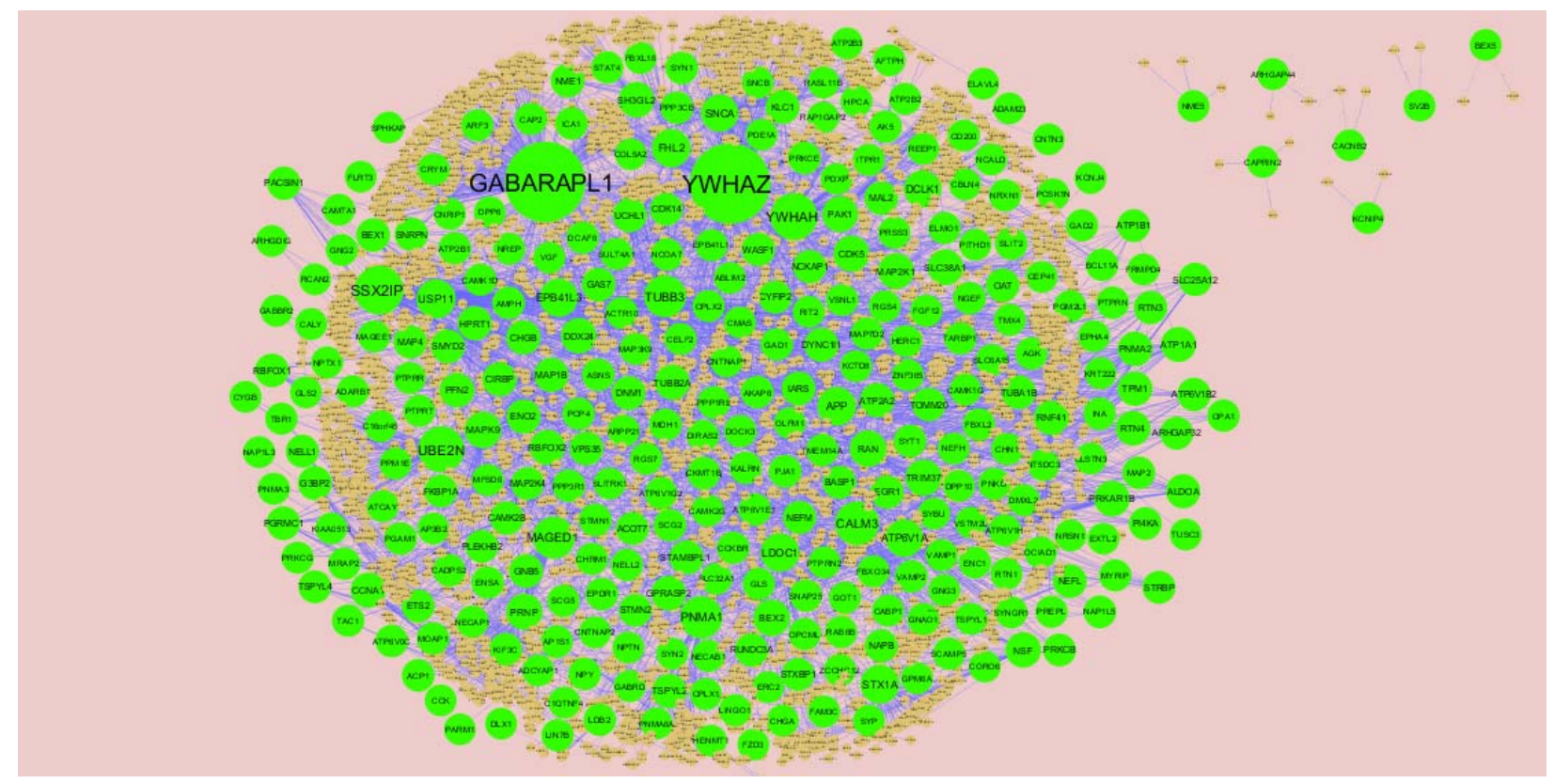

Fig. 5. Protein-protein interaction network of differentially expressed genes (DEGs). Green nodes denotes up regulated genes.

A

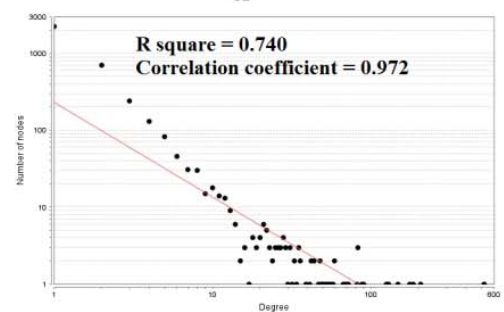

D

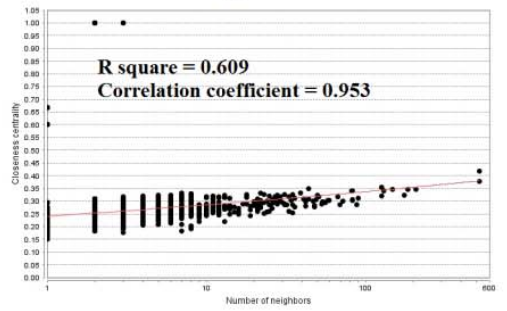

B

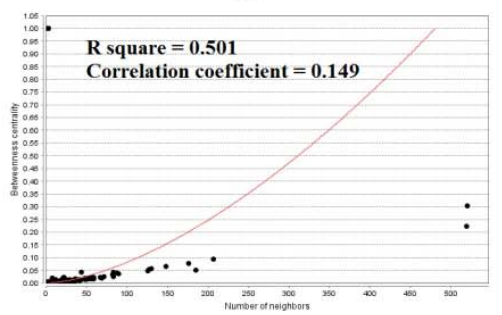

E

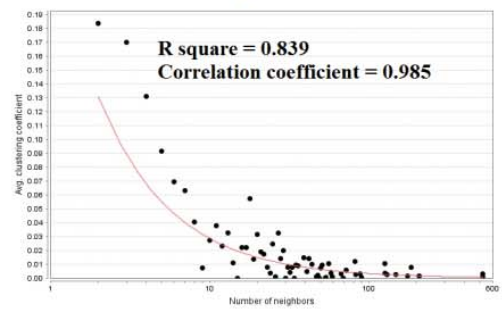

C

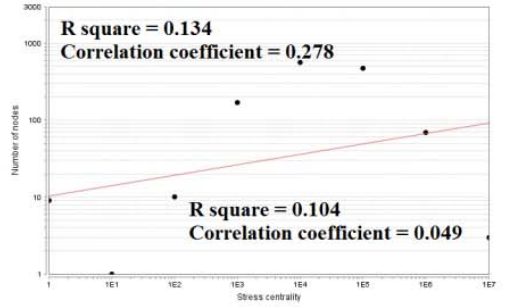

Fig. 6. Scatter plot for up regulated genes. (A- Node degree; B- Betweenness centrality; C- Stress centrality ; DCloseness centrality; E- Clustering coefficient) 

medRxiv preprint doi: https://doi.org/10.1101/2020.12.21.20248688; this version posted December 24,2020 . The copyright holder for this
preprint (which was not certified bv peer review) is the author/funder. who has aranted medRxiv a license to displav the preprint in perpetuitv. medRxiv preprint doi: https://doi.org/10.1101/2020.12.21.20248688; this version posted December 24,2020 . The copyright holder for this
preprint (which was not certified by peer review) is the author/funder, who has granted medRxiv a license to display the preprint in perpetuity. All rights reserved. No reuse allowed without permission.

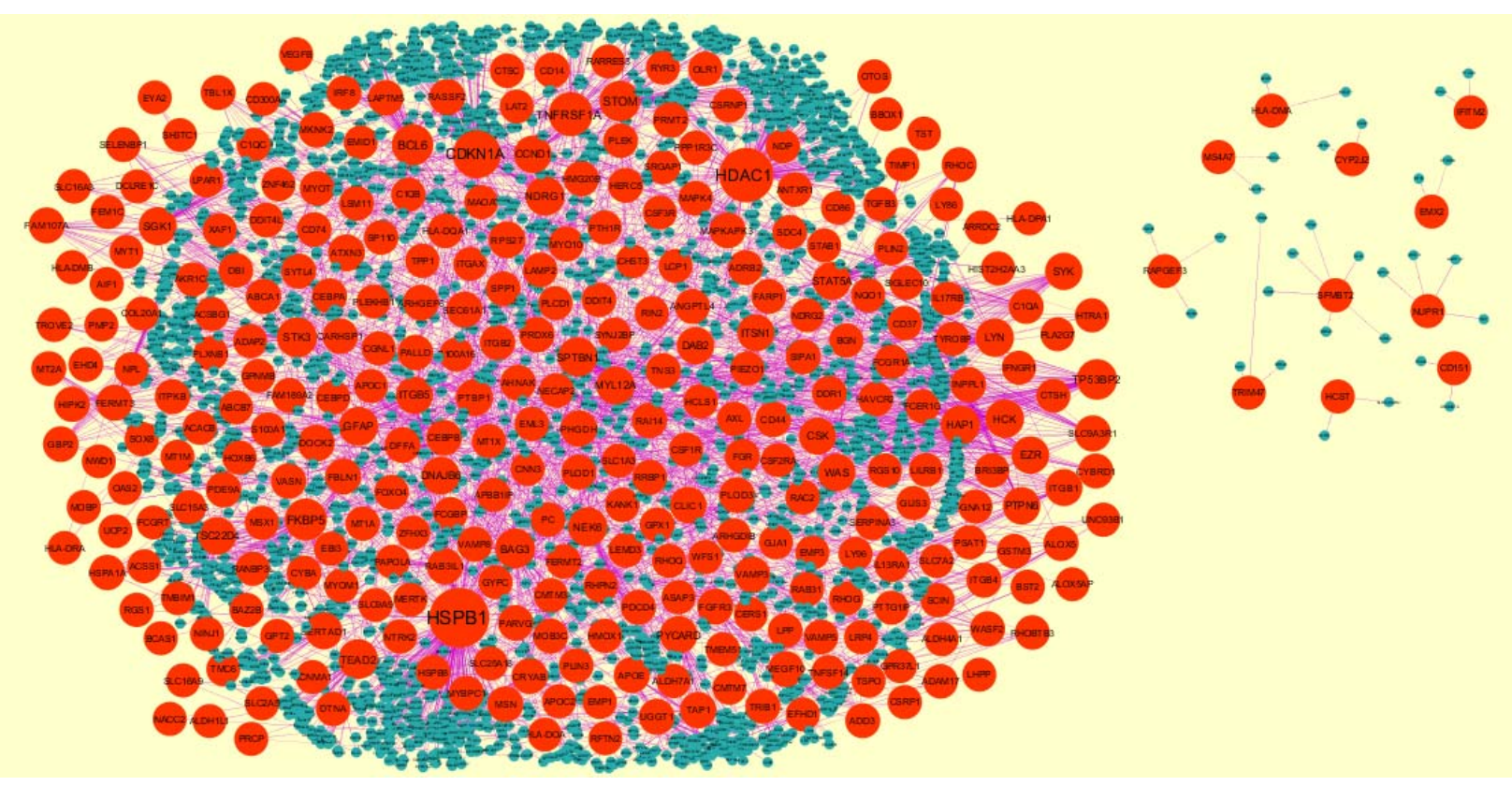

Fig. 7. Protein-protein interaction network of differentially expressed genes (DEGs). Red nodes denotes down regulated genes.

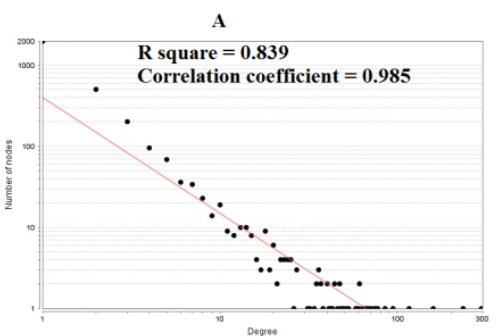

D

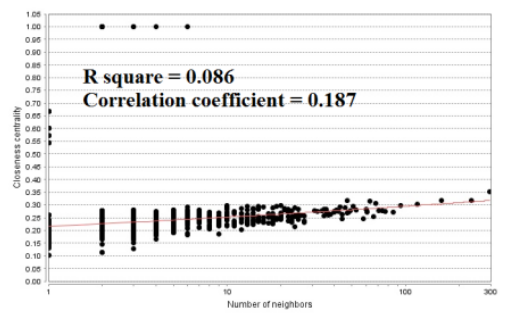

B
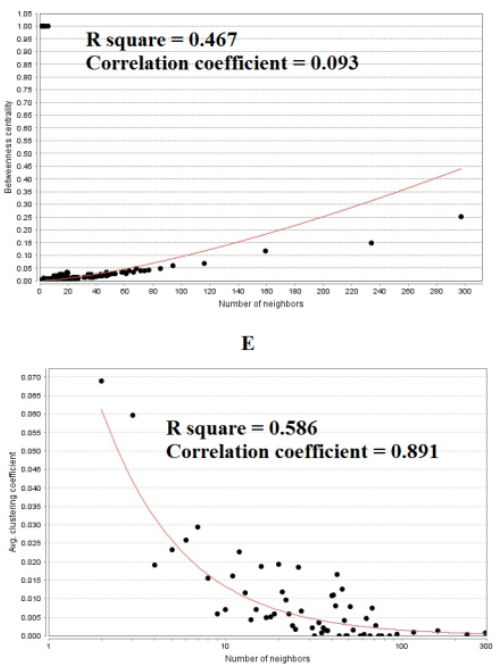

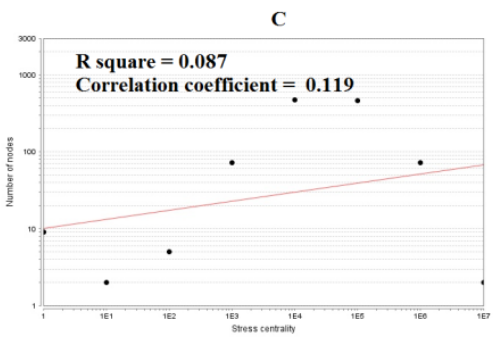

Fig. 8. Scatter plot for down regulated genes. (A - Node degree; B - Betweenness centrality; C- Stress centrality ;DCloseness centrality; E - Clustering coefficient) 
medRxiv preprint doi: https://doi.org/10.1101/2020.12.21.20248688; this version posted December 24, 2020. The copyright holder for this preprint (which was not certified by peer review) is the author/funder, who has granted medRxiv a license to display the preprint in perpetuity.

All rights reserved. No reuse allowed without permission.

\section{Module 1}

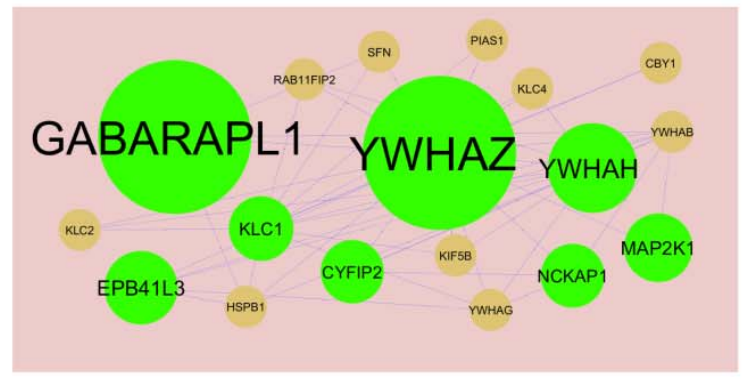

Module 6

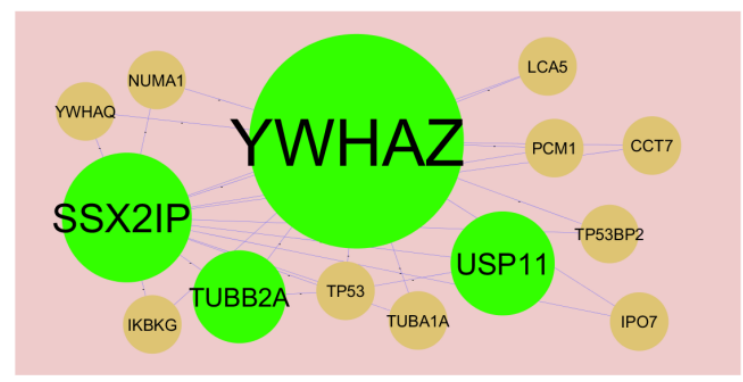

Module 3

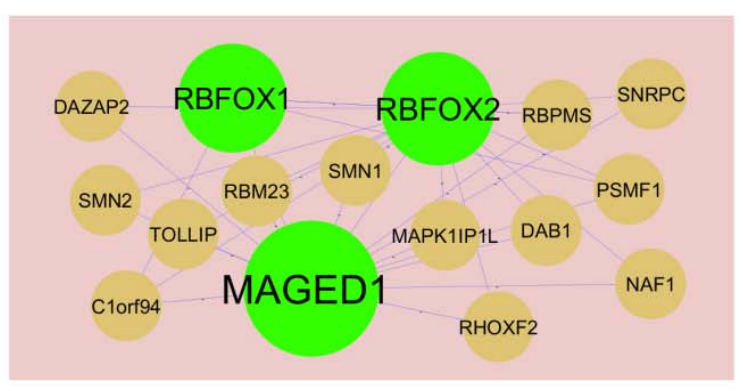

Module 29

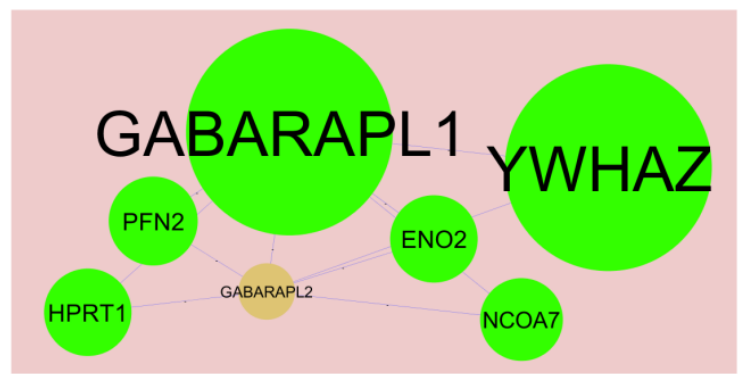

Fig. 9. Modules in PPI network. The green nodes denote the up regulated genes 


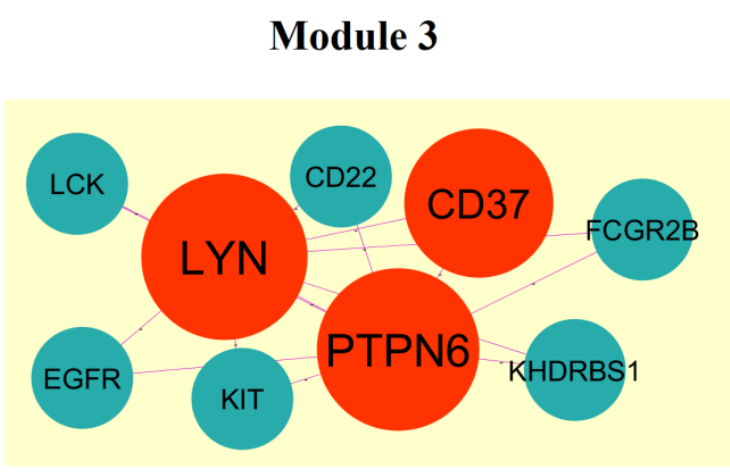

Module 9

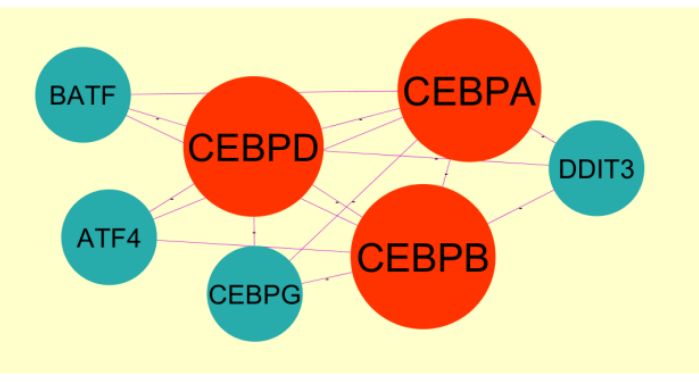

Module 5

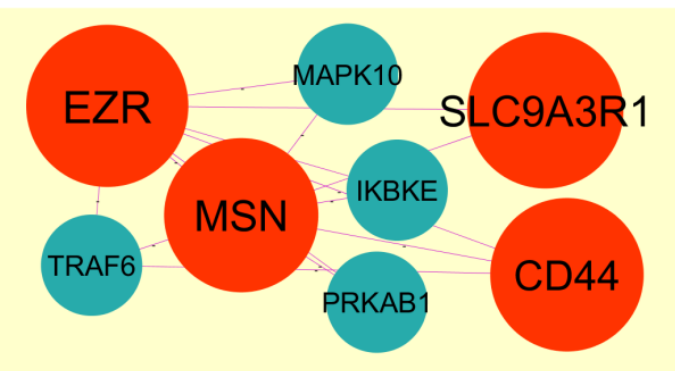

Module 10

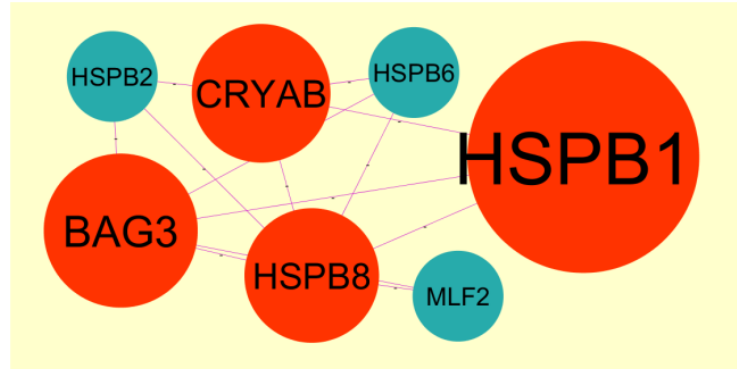

Fig. 10. Modules in PPI network. The red nodes denote the down regulated genes. 
medRxiv preprint doi: https://doi.org/10.1101/2020.12.21.20248688; this version posted December 24, 2020. The copyright holder for this preprint (which was not certified by peer review) is the author/funder, who has granted medRxiv a license to display the preprint in perpetuity. All rights reserved. No reuse allowed without permission.

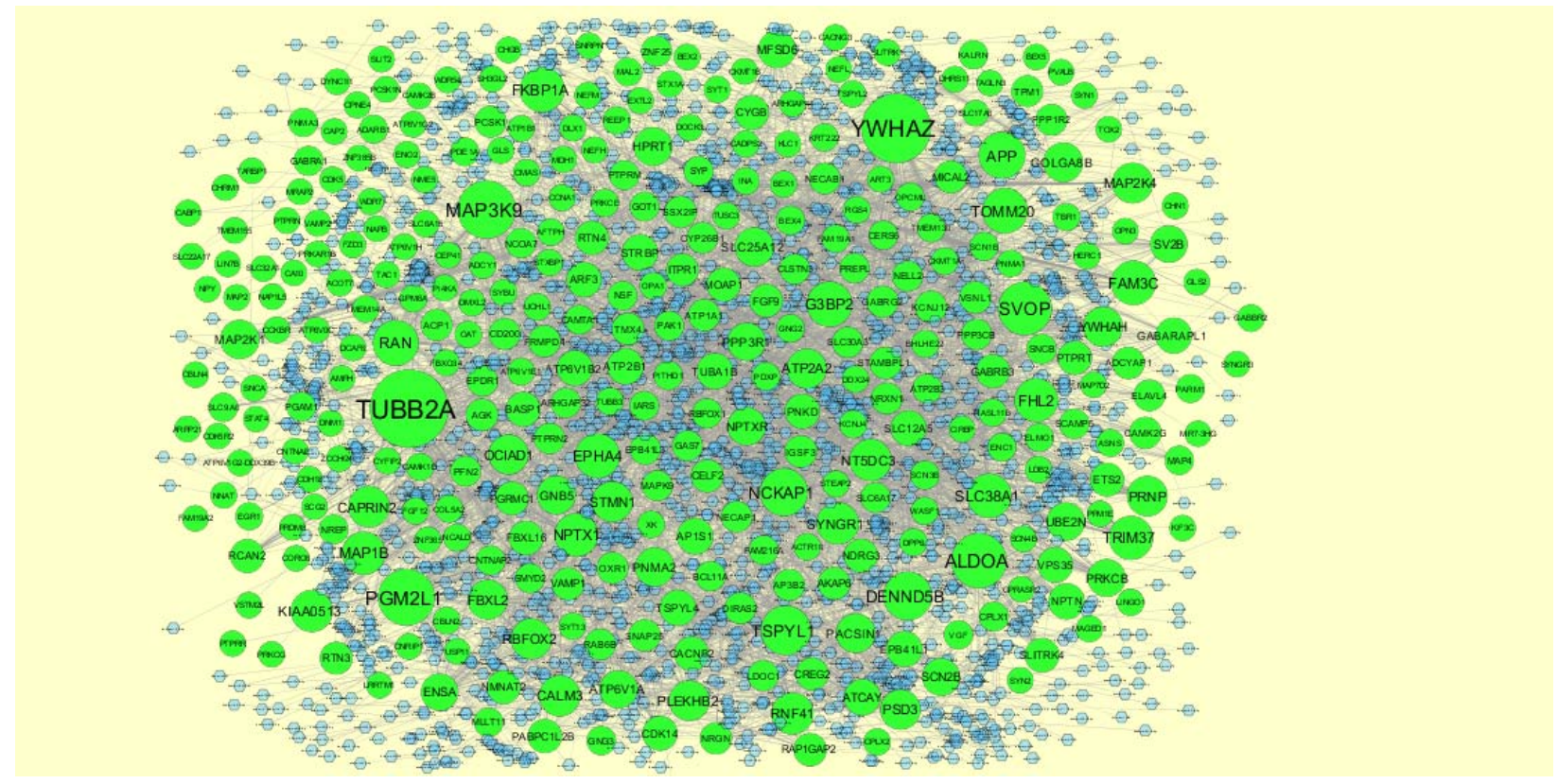

Fig. 11. The network of up regulated DEGs and their related miRNAs. The green circles nodes are the up regulated DEGs, and blue diamond nodes are the miRNAs 
medRxiv preprint doi: https://doi.org/10.1101/2020.12.21.20248688; this version posted December 24, 2020. The copyright holder for this preprint (which was not certified by peer review) is the author/funder, who has granted medRxiv a license to display the preprint in perpetuity. All rights reserved. No reuse allowed without permission.

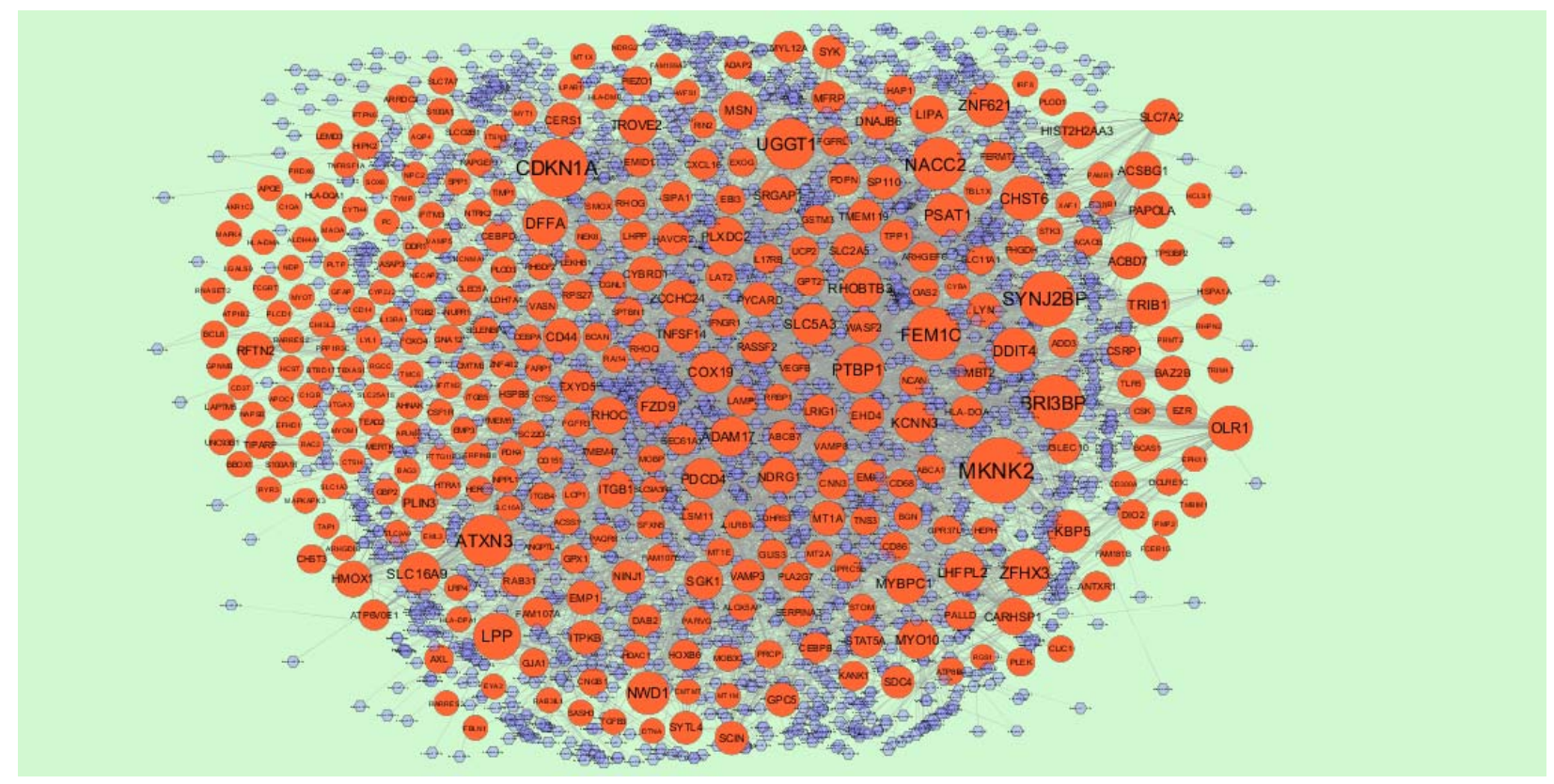

Fig. 12. The network of down regulated DEGs and their related miRNAs. The red circles nodes are the downregulated DEGs, and lavender diamond nodes are the miRNAs 
medRxiv preprint doi: https://doi.org/10.1101/2020.12.21.20248688; this version posted December 24, 2020. The copyright holder for this preprint (which was not certified by peer review) is the author/funder, who has granted medRxiv a license to display the preprint in perpetuity. All rights reserved. No reuse allowed without permission.

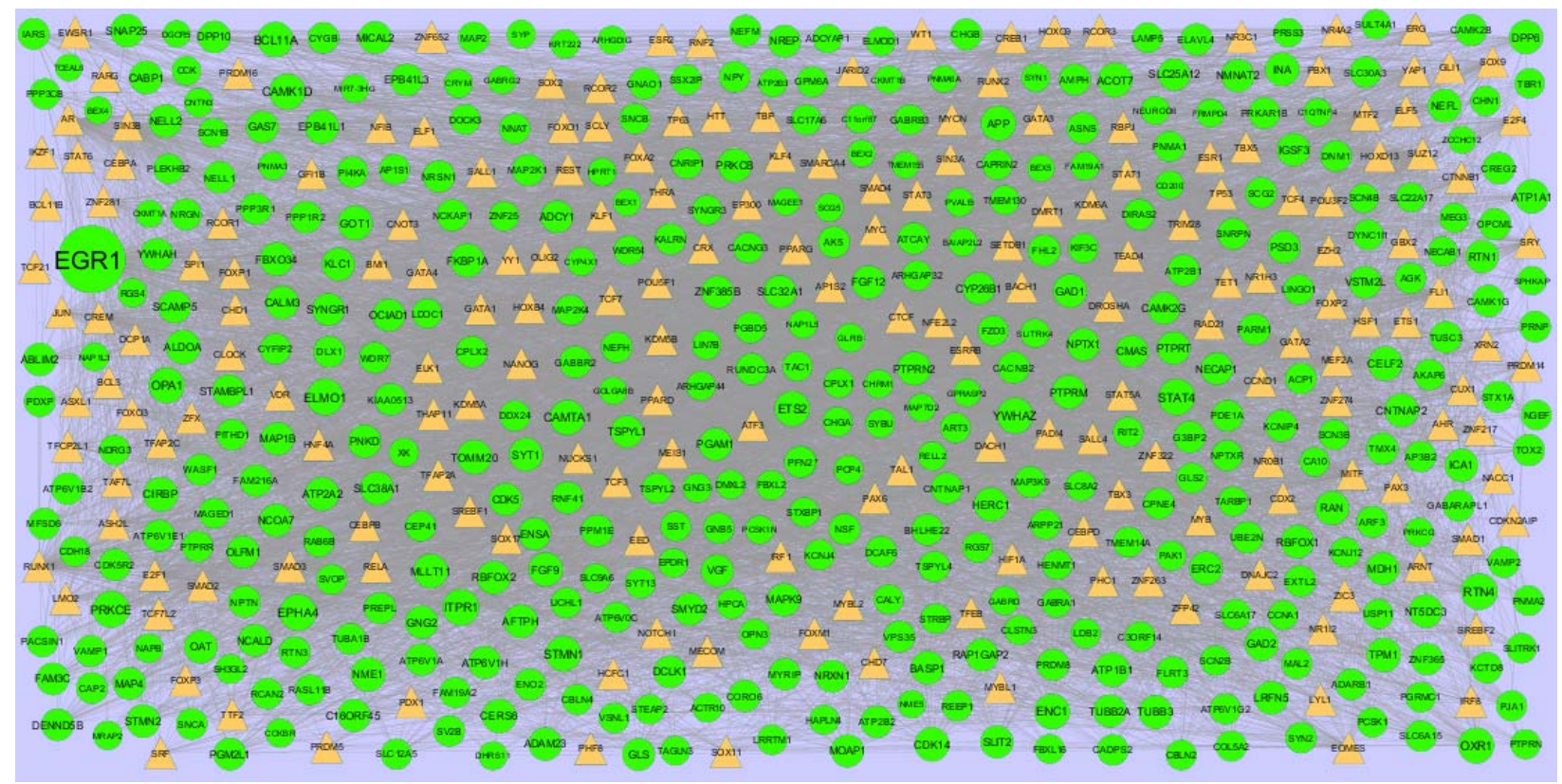

Fig. 13. TF-gene network of predicted target up regulated genes. (Orange triangle - TFs and green circles- target up regulated genes)

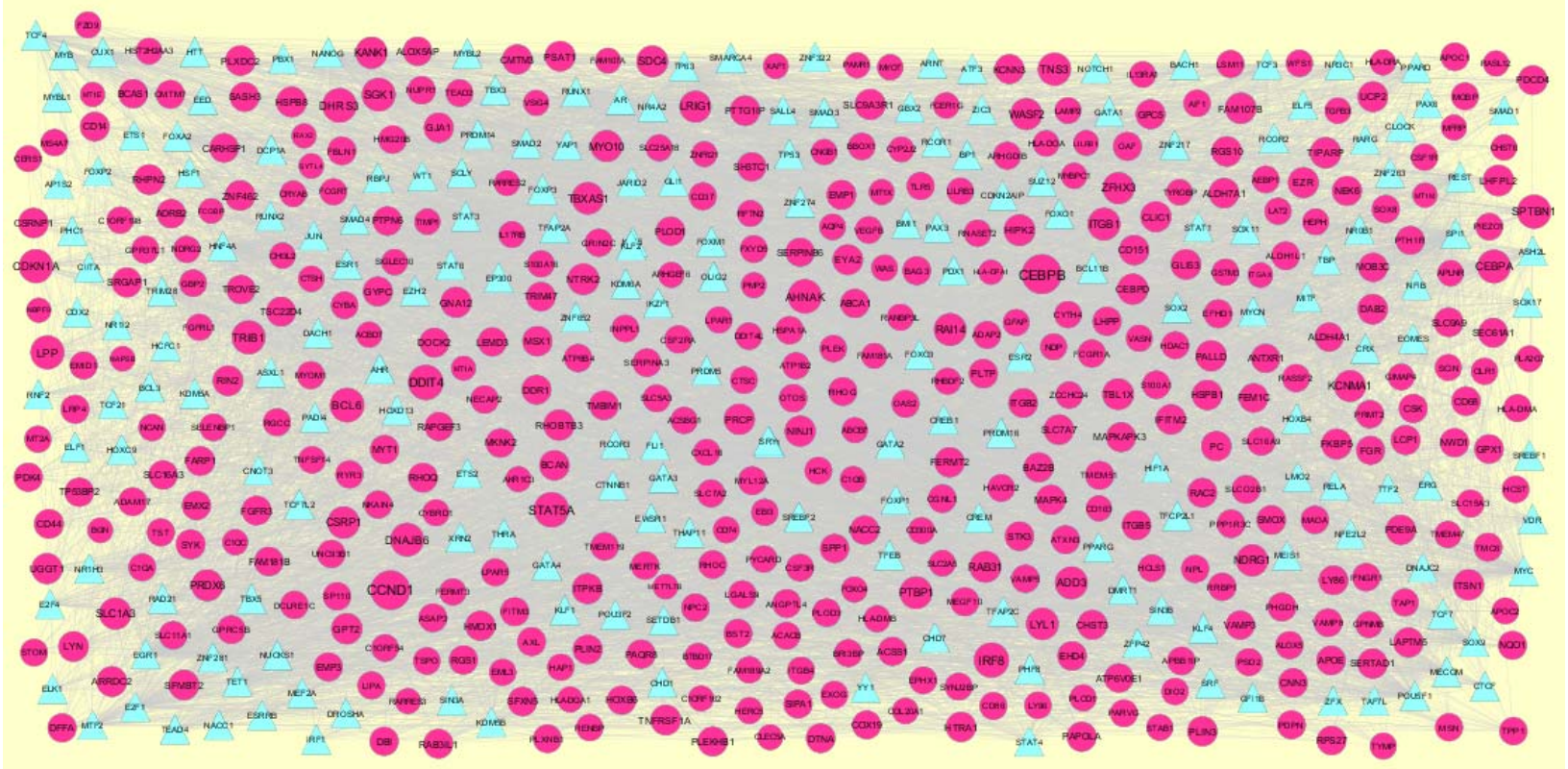

Fig. 14. TF-gene network of predicted target down regulated genes. (Blue triangle - TFs and pink circles- target up regulated genes) 
medRxiv preprint doi: https://doi.org/10.1101/2020.12.21.20248688; this version posted December 24, 2020. The copyright holder for this preprint (which was not certified by peer review) is the author/funder, who has granted medRxiv a license to display the preprint in perpetuity. All rights reserved. No reuse allowed without permission.
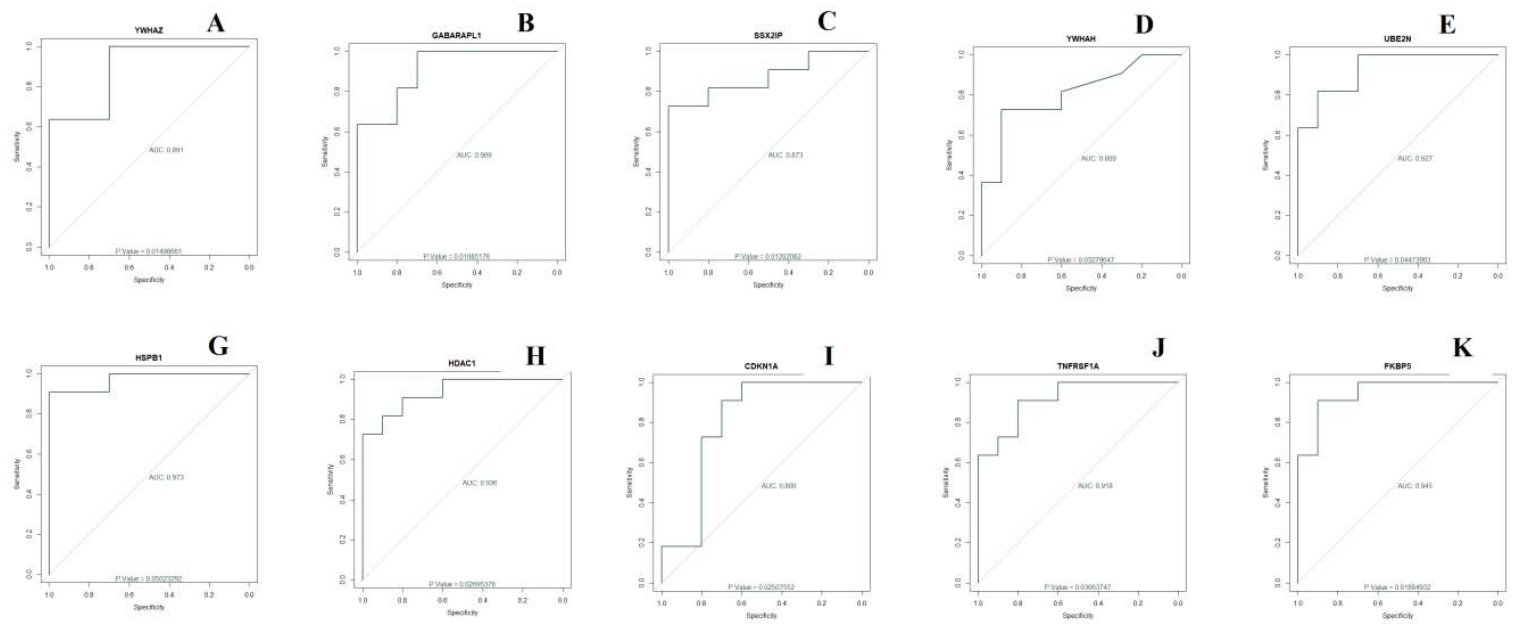

Fig. 15. ROC curve validated the sensitivity, specificity of hub genes as a predictive biomarker for sCJD prognosis. A) YWHAZ, B) GABARAPL1, C) SSX2IP, D) YWHAH, E) UBE2N, F) HSPB1, G) HDAC1, H) CDKN1A, I) TNFRSF1A and J) FKBP5 